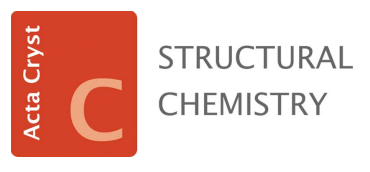

ISSN 2053-2296

\title{
Design of two series of 1:1 cocrystals involving 4-amino-5-chloro-2,6-dimethylpyrimidine and carboxylic acids
}

\author{
Ammaiyappan Rajam, ${ }^{\text {a Packianathan Thomas Muthiah, }}{ }^{\text {a* }}$ Raymond John Butcher, ${ }^{\mathrm{b}}$ \\ Jerry P. Jasinski ${ }^{\mathrm{C}}$ and Jan Wikaira ${ }^{\mathrm{d}}$ \\ aSchool of Chemistry, Bharathidasan University, Tiruchirappalli 620 024, Tamilnadu, India, 'bepartment of Chemistry,
Howard University, 525 College Street NW, Washington, DC 20059, USA, 'Department of Chemistry, Keene State
College, 229 Main Street, Keene, NH 03435-2001, USA, and ${ }^{\mathbf{d} D e p a r t m e n t ~ o f ~ C h e m i s t r y, ~ U n i v e r s i t y ~ o f ~ C a n t e r b u r y, ~}$
Private Bag 4800, Christchurch, New Zealand. *Correspondence e-mail: tommtrichy@yahoo.co.in
}

Received 11 May 2018

Accepted 25 June 2018

Edited by A. L. Spek, Utrecht University, The Netherlands

Keywords: cocrystal; pyrimidine; carboxylic acid; protonation; supramolecular structure; heterosynthon; hydrogen bonding; crystal structure.

CCDC references: $1851467 ; 1851466$; $1851465 ; 1851464 ; 1851463 ; 1851462$; $1851461 ; 1851460 ; 1851459 ; 1851458$

Supporting information: this article has supporting information at journals.iucr.org/c

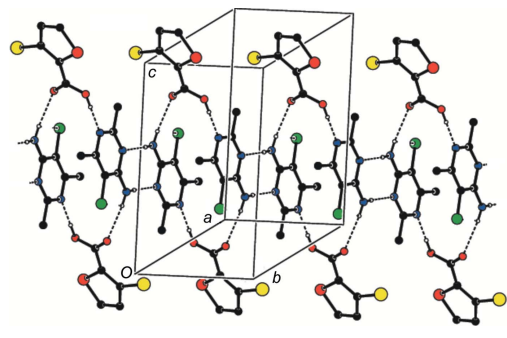

C 2018 International Union of Crystallography
Two series of a total of ten cocrystals involving 4-amino-5-chloro-2,6-dimethylpyrimidine with various carboxylic acids have been prepared and characterized by single-crystal X-ray diffraction. The pyrimidine unit used for the cocrystals offers two ring $\mathrm{N}$ atoms (positions $\mathrm{N} 1$ and $\mathrm{N} 3$ ) as proton-accepting sites. Depending upon the site of protonation, two types of cations are possible [Rajam et al. (2017). Acta Cryst. C73, 862-868]. In a parallel arrangement, two series of cocrystals are possible depending upon the hydrogen bonding of the carboxyl group with position $\mathrm{N} 1$ or N3. In one series of cocrystals, i.e. 4-amino-5chloro-2,6-dimethylpyrimidine-3-bromothiophene-2-carboxylic acid (1/1), 1, 4-amino-5-chloro-2,6-dimethylpyrimidine-5-chlorothiophene-2-carboxylic acid (1/1), 2, 4-amino-5-chloro-2,6-dimethylpyrimidine-2,4-dichlorobenzoic acid (1/1), 3, and 4-amino-5-chloro-2,6-dimethylpyrimidine-2-aminobenzoic acid $(1 / 1), \mathbf{4}$, the carboxyl hydroxy group $(-\mathrm{OH})$ is hydrogen bonded to position $\mathrm{N} 1(\mathrm{O}-\mathrm{H} \cdots \mathrm{N} 1)$ of the corresponding pyrimidine unit (single point supramolecular synthon). The inversion-related stacked pyrimidines are doubly bridged by the carboxyl groups via $\mathrm{N}-\mathrm{H} \cdots \mathrm{O}$ and $\mathrm{O}-\mathrm{H} \cdots \mathrm{N}$ hydrogen bonds to form a large cage-like tetrameric unit with an $R_{4}^{2}(20)$ graph-set ring motif. These tetrameric units are further connected via base pairing through a pair of $\mathrm{N}-\mathrm{H} \cdots \mathrm{N}$ hydrogen bonds, generating $R_{2}^{2}(8)$ motifs (supramolecular homosynthon). In the other series of cocrystals, i.e. 4-amino-5-chloro-2,6-dimethylpyrimidine-5-methylthiophene-2-carboxylic acid (1/1), 5, 4-amino-5-chloro-2,6dimethylpyrimidine-benzoic acid (1/1), 6, 4-amino-5-chloro-2,6-dimethylpyrimidine-2-methylbenzoic acid (1/1), 7, 4-amino-5-chloro-2,6-dimethylpyrimidine3-methylbenzoic acid (1/1), 8, 4-amino-5-chloro-2,6-dimethylpyrimidine4-methylbenzoic acid (1/1), 9, and 4-amino-5-chloro-2,6-dimethylpyrimidine4-aminobenzoic acid (1/1), 10, the carboxyl group interacts with position N3 and the adjacent 4-amino group of the corresponding pyrimidine ring via $\mathrm{O}-\mathrm{H} \cdots \mathrm{N}$ and $\mathrm{N}-\mathrm{H} \cdots \mathrm{O}$ hydrogen bonds to generate the robust $R_{2}^{2}(8)$ supramolecular heterosynthon. These heterosynthons are further connected by $\mathrm{N}-\mathrm{H} \cdots \mathrm{N}$ hydrogen-bond interactions in a linear fashion to form a chain-like arrangement. In cocrystal $\mathbf{1}$, a $\mathrm{Br} \cdots \mathrm{Br}$ halogen bond is present, in cocrystals $\mathbf{2}$ and $\mathbf{3}, \mathrm{Cl} \cdots \mathrm{Cl}$ halogen bonds are present, and in cocrystals 5,6 and $7, \mathrm{Cl} \cdots \mathrm{O}$ halogen bonds are present. In all of the ten cocrystals, $\pi-\pi$ stacking interactions are observed.

\section{Introduction}

In crystal engineering, noncovalent interactions such as hydrogen bonds, halogen bonds, anion $-\pi$, cation $-\pi$ and $\pi-\pi$ interactions, together with other weak forces, play a central role in the self-assembly and molecular recognition processes (García-Raso et al., 2009; Aakeröy et al., 2009; Nandi et al., 2014). In particular, halogen bonding is of current interest and exhibits a wide range of applications, such as in catalysis, 
medicinal chemistry and structural chemistry (Cavallo et al., 2016; Metrangolo \& Resnati, 2012). Recently, much consideration has been given to the synthesis of cocrystals related to their wide-ranging applications in the pharmaceutical industry (Zhu et al., 2015; Griffini et al., 2014; Baldrighi et al., 2013). Pyrimidines and aminopyrimidine derivatives are biologically important compounds as they are distributed widely in nature as components of nucleic acids and drugs (Selvam et al., 2012; Sharma et al., 2014). In crystal engineering, carboxylic acid is one of the most commonly used functional groups and hence the formation of robust heterosynthons from the carboxylic acid-pyrimidine moiety has attracted much attention. The pyrimidine-carboxylic acid interaction is also involved in protein-nucleic acid binding and drug-protein recognition processes. Hydrogen-bonding patterns, including base pairing formed by the aminopyrimidines, are important in the structure and functions of nucleic acids (Stanley et al., 2002; Baskar Raj et al., 2003). The hydrogen-bonding patterns present in several aminopyrimidine-carboxylates and cocrystals have been reported by us (Ebenezer et al., 2011; Tamilselvi \& Muthiah, 2011; Ebenezer \& Muthiah, 2012; Jegan Jennifer \& Muthiah, 2014; Karthikeyan et al., 2015; Rajam et al., 2015, 2017; Swinton Darious et al., 2017).

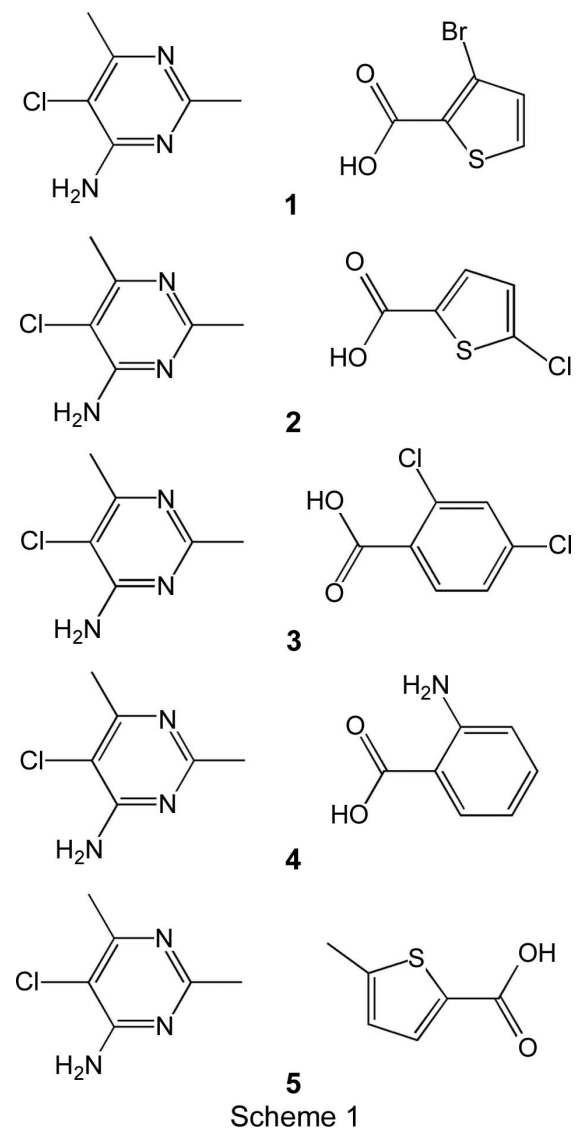

In the present study, we have synthesized and structurally characterized two series of a total of ten cocrystals, namely 4-amino-5-chloro-2,6-dimethylpyrimidine-3-bromothiophene2-carboxylic acid (1/1), 1, 4-amino-5-chloro-2,6-dimethylpy- rimidine-5-chlorothiophene-2-carboxylic acid (1/1), $\mathbf{2}$, 4-amino-5-chloro-2,6-dimethylpyrimidine-2,4-dichlorobenzoic acid (1/1), 3, 4-amino-5-chloro-2,6-dimethylpyrimidine-2-aminobenzoic acid (1/1), 4, 4-amino-5-chloro-2,6-dimethylpyrimidine5-methylthiophene-2-carboxylic acid (1/1), 5, 4-amino-5-chloro2,6-dimethylpyrimidine-benzoic acid (1/1), 6, 4-amino-5-chloro2,6-dimethylpyrimidine-2-methylbenzoic acid (1/1), 7, 4-amino5-chloro-2,6-dimethylpyrimidine-3-methylbenzoic acid (1/1), $\mathbf{8}$, 4-amino-5-chloro-2,6-dimethylpyrimidine-4-methylbenzoic acid (1/1), 9, and 4-amino-5-chloro-2,6-dimethylpyrimidine-4-aminobenzoic acid (1/1), 10. Depending on the nature and position of the substituent present in the carboxylic acids, the carboxyl hydroxy group $(-\mathrm{OH})$ is hydogen bonded to atom $\mathrm{N} 1(\mathrm{O}-$ $\mathrm{H} \cdots \mathrm{N} 1)$ of the corresponding pyrimidine ring, i.e. crystal structures 1-4. Atom N1 is flanked by the methyl groups present in the pyrimidine moiety, so a robust heterosynthon motif formation is not possible. For crystal structures 5-10, the carboxyl group interacts with atom $\mathrm{N} 3$ and the adjacent 4-amino group of the corresponding pyrimidine ring via $\mathrm{O}-$ $\mathrm{H} \cdots \mathrm{N}$ and $\mathrm{N}-\mathrm{H} \cdots \mathrm{O}$ hydrogen bonds to generate the robust $R_{2}^{2}(8)$ supramolecular heterosynthon.
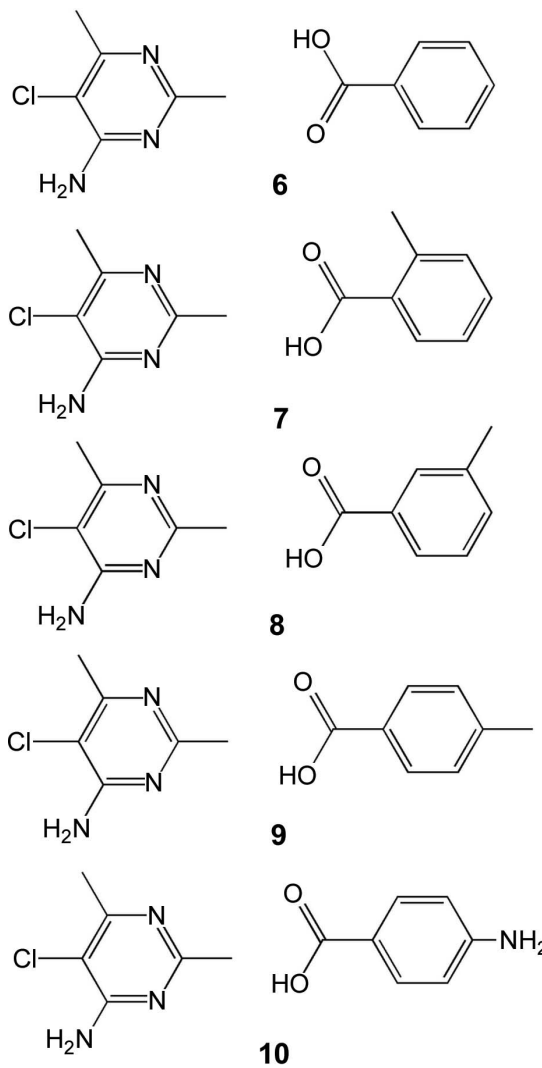

9

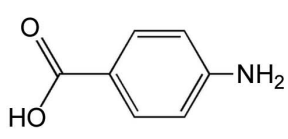

10

Scheme 2

\section{Experimental}

\subsection{Synthesis and crystallization}

2.1.1. Cocrystal 1. 4-Amino-5-chloro-2,6-dimethylpyrimidine $(39 \mathrm{mg})$ and 3-bromothiophene-2-carboxylic acid $(51 \mathrm{mg})$ were dissolved in a hot acetonitrile solution (1:1 molar ratio) and warmed over a water bath for half an hour. The resulting clear solution was allowed to evaporate. After a few days, 
Table 1

Experimental details.

For all determinations, $\mathrm{H}$ atoms were treated by a mixture of independent and constrained refinement.

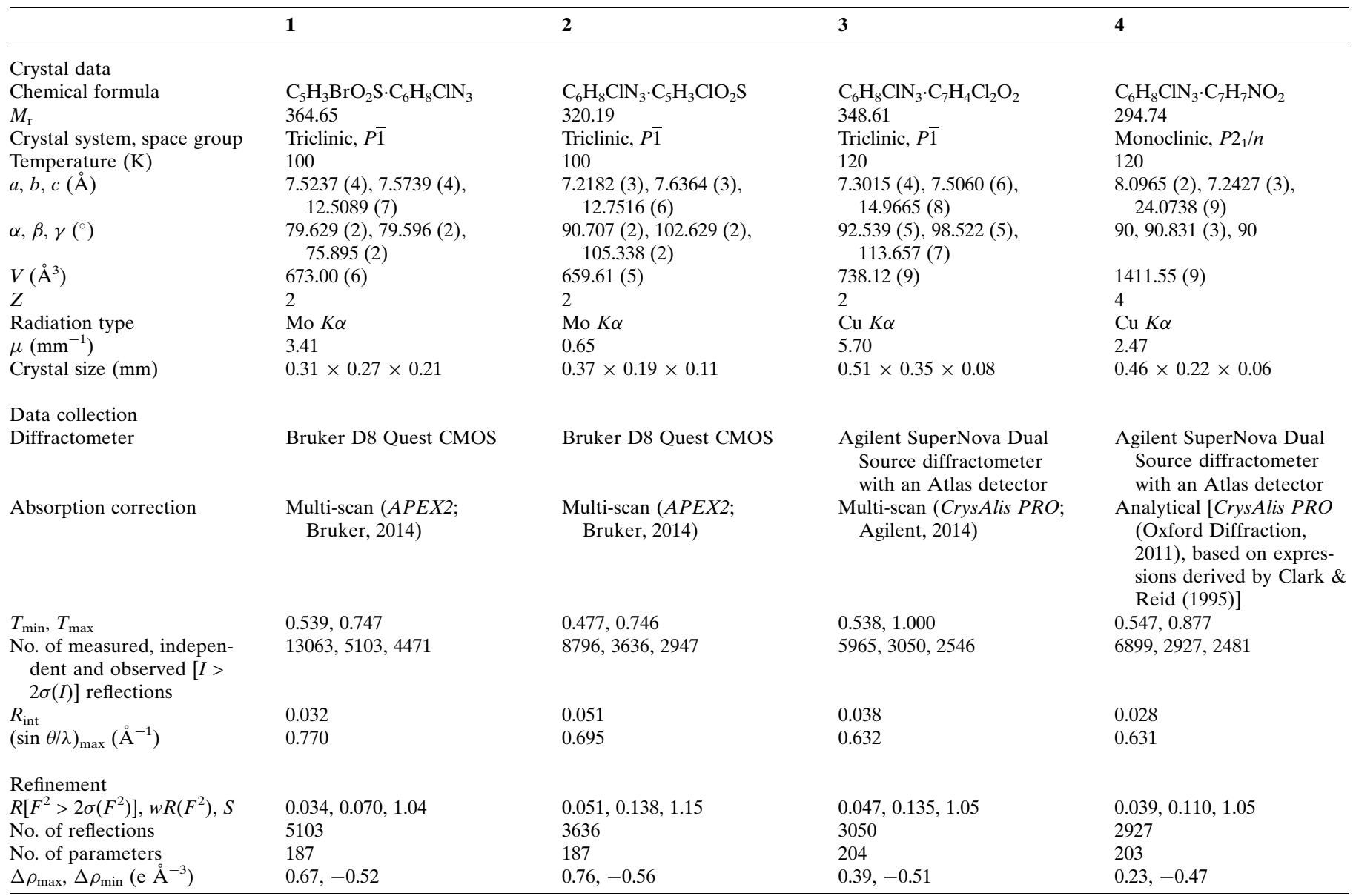

5

Crystal data

Chemical formula

$M_{\mathrm{r}}$

Crystal system, space group

Temperature $(\mathrm{K})$

$a, b, c(\AA)$

$\alpha, \beta, \gamma\left(^{\circ}\right)$

$V\left(\AA^{3}\right)$

Z

Radiation type

$\mu\left(\mathrm{mm}^{-1}\right)$

Crystal size (mm)

Data collection

Diffractometer

Absorption correction

$T_{\min }, T_{\max }$

No. of measured, independent and observed $[I>$ $2 \sigma(I)]$ reflections

$R_{\text {int }}$

$(\sin \theta / \lambda)_{\max }\left(\AA^{-1}\right)$

0.028

Refinemen

$R\left[F^{2}>2 \sigma\left(F^{2}\right)\right], w R\left(F^{2}\right), S$

No. of reflections
0.028
0.734

\section{$\mathrm{C}_{6} \mathrm{H}_{8} \mathrm{ClN}_{3} \cdot \mathrm{C}_{6} \mathrm{H}_{6} \mathrm{O}_{2} \mathrm{~S}$ 299.77 \\ Monoclinic, $P 2_{1} / c$ \\ 100 \\ 8.273 (3), 12.746 (4), \\ $13.320(4)$ \\ 90, $102.390(5), 90$ \\ $1371.8(8)$ \\ Mo $K \alpha$ \\ 0.43 \\ $0.55 \times 0.50 \times 0.30$}

\section{Bruker APEXII CCD}

Multi-scan (APEX2;

Bruker, 2014)

$0.608,0.746$

$8807,4109,3735$

$0.034,0.092,1.05$

4109

\section{$\mathrm{C}_{6} \mathrm{H}_{8} \mathrm{ClN}_{3} \cdot \mathrm{C}_{7} \mathrm{H}_{6} \mathrm{O}_{2}$} 279.72

Monoclinic, $P 2_{1} / c$

120

10.1421 (5), 10.4218 (5),

$13.2681(6)$

90, $107.804(5), 90$

1335.26 (12)

$\mathrm{Cu} K \alpha$

2.56

$0.52 \times 0.38 \times 0.24$

Agilent SuperNova Dual Source diffractometer with an Atlas detector Multi-scan (CrysAlis PRO;

Agilent, 2014)

$0.763,1.000$

$6087,2760,2560$

0.029

0.631

$0.041,0.118,1.04$

2760

78

8

$\begin{array}{ll}\mathrm{C}_{6} \mathrm{H}_{8} \mathrm{ClN}_{3} \cdot \mathrm{C}_{8} \mathrm{H}_{8} \mathrm{O}_{2} & \mathrm{C}_{6} \mathrm{H}_{8} \mathrm{ClN}_{3} \cdot \mathrm{C}_{8} \mathrm{H}_{8} \mathrm{O}_{2} \\ 293.75 & 293.75 \\ \text { Orthorhombic, } P 2_{1} 2_{1} 2_{1} & \text { Orthorhombic, Pbcn } \\ 120 & 120 \\ 7.3992(2), 13.9842(4), & 8.4524(1), 13.5387(2), \\ \quad 26.9897(8) & 24.9813(3) \\ 90,90,90 & 90,90,90 \\ 2792.68(14) & 2858.72(6) \\ 8 & 8 \\ \mathrm{Cu} \mathrm{K \alpha} & \mathrm{Cu} \mathrm{K \alpha} \\ 2.47 & 2.42 \\ 0.49 \times 0.11 \times 0.08 & 0.42 \times 0.24 \times 0.19\end{array}$

Agilent SuperNova Dual Source diffractometer with an Atlas detector Multi-scan (CrysAlis PRO; Agilent, 2014)

$0.740,1.000$

$8897,4966,4617$

Agilent SuperNova Dual Source diffractometer with an Atlas detector Multi-scan (CrysAlis PRO;

Agilent, 2014)

$0.680,1.000$

26401, 3006, 2904

$0.030 \quad 0.033$

$0.631 \quad 0.631$

$0.034,0.091,1.04 \quad 0.037,0.103,1.04$

4966

3006 
Table 1 (continued)

\begin{tabular}{lllll}
\hline & $\mathbf{5}$ & $\mathbf{6}$ & $\mathbf{7}$ & $\mathbf{8}$ \\
\hline $\begin{array}{l}\text { No. of parameters } \\
\Delta \rho_{\text {max }} \Delta \rho_{\text {min }}\left(\mathrm{e}^{-3}\right)\end{array}$ & 189 & 187 & 376 & 196 \\
Absolute structure & $0.55,-0.29$ & $0.33,-0.44$ & $0.24,-0.24$ & $0.30,-0.31$ \\
& - & - & Flack $x$ determined using & - \\
& & & 1451 quotients & {$\left[\left(I^{+}\right)-\left(I^{-}\right)\right] /\left[\left(I^{+}\right)+\left(I^{-}\right)\right]$} \\
$\begin{array}{l}\text { Absolute structure para- } \\
\text { meter }\end{array}$ & - & - & -0.001 (10) et al. 2013) & - \\
\hline
\end{tabular}

\begin{tabular}{|c|c|c|}
\hline \multicolumn{3}{|l|}{ Crystal data } \\
\hline Chemical formula & $\mathrm{C}_{6} \mathrm{H}_{8} \mathrm{ClN}_{3} \cdot \mathrm{C}_{8} \mathrm{H}_{8} \mathrm{O}_{2}$ & $\mathrm{C}_{6} \mathrm{H}_{8} \mathrm{ClN}_{3} \cdot \mathrm{C}_{7} \mathrm{H}_{7} \mathrm{NO}_{2}$ \\
\hline Crystal system, space group & Monoclinic, $P 2_{1} / c$ & Monoclinic, $P 2_{1} / c$ \\
\hline Temperature $(\mathrm{K})$ & 120 & 120 \\
\hline$a, b, c(\AA)$ & $8.0223(5), 13.6901(10), 13.3346(10)$ & $7.8899(2), 13.2361(3), 13.3212(3)$ \\
\hline$\alpha, \beta, \gamma\left({ }^{\circ}\right)$ & $90,101.557$ (7), 90 & $90,101.254(2), 90$ \\
\hline Radiation type & $\mathrm{Cu} K \alpha$ & $\mathrm{Cu} K \alpha$ \\
\hline$\mu\left(\mathrm{mm}^{-1}\right)$ & 2.41 & 2.56 \\
\hline Crystal size $(\mathrm{mm})$ & $0.51 \times 0.17 \times 0.08$ & $0.41 \times 0.35 \times 0.24$ \\
\hline \multicolumn{3}{|l|}{ Data collection } \\
\hline Diffractometer & $\begin{array}{l}\text { Agilent SuperNova Dual Source diffractometer } \\
\text { with an Atlas detector }\end{array}$ & $\begin{array}{l}\text { Agilent SuperNova Dual Source diffractometer } \\
\text { with an Atlas detector }\end{array}$ \\
\hline$(\sin \theta / \lambda)_{\max }\left(\AA^{-1}\right)$ & 0.631 & 0.631 \\
\hline \multicolumn{3}{|l|}{ Refinement } \\
\hline$R\left[F^{2}>2 \sigma\left(F^{2}\right)\right], w R\left(F^{2}\right), S$ & $0.047,0.140,1.04$ & $0.036,0.097,1.04$ \\
\hline No. of reflections & 2966 & 2823 \\
\hline No. of parameters & 197 & 203 \\
\hline$\Delta \rho_{\max }, \Delta \rho_{\min }\left(\mathrm{e} \AA^{-3}\right)$ & $0.28,-0.41$ & $0.27,-0.38$ \\
\hline
\end{tabular}

Computer programs: APEX2 (Bruker, 2014), CrysAlis PRO (Agilent, 2014; Oxford Diffraction, 2011), SAINT (Bruker, 2013, 2014), SHELXS97 (Sheldrick, 2008), SHELXT (Sheldrick, 2015a), SHELXL2018 (Sheldrick, 2015b), SHELXL2014 (Sheldrick, 2015b), shelXle (Hübschle et al., 2011), SHELXTL (Sheldrick, 2008), Mercury (Macrae et al., 2008) and PLATON (Spek, 2009).

colourless block-shaped crystals of $\mathbf{1}$ separated out from the mother liquor.

2.1.2. Cocrystal 2. 4-Amino-5-chloro-2,6-dimethylpyrimidine (39 mg) and 5-chlorothiophene-2-carboxylic acid (41 mg) were dissolved in a hot dimethylformamide (DMF) solution (1:1 molar ratio) and warmed over a water bath for half an hour. The clear solution was allowed to evaporate. After a few days, colourless block-shaped crystals of $\mathbf{2}$ separated out from the mother liquid.

2.1.3. Cocrystal 3. 4-Amino-5-chloro-2,6-dimethylpyrimidine (39 mg) and 2,4-dichlorobenzoic acid (47 mg) were dissolved in hot ethanol (1:1 molar ratio) and warmed over a water bath for half an hour. The clear solution was allowed to evaporate. After a few days, colourless crystals of $\mathbf{3}$ separated out from the mother liquid.

2.1.4. Cocrystal 4. 4-Amino-5-chloro-2,6-dimethylpyrimidine (39 $\mathrm{mg}$ ) and 2-aminobenzoic acid (34 mg) were dissolved in hot ethanol (1:1 molar ratio) and warmed over a water bath for half an hour. The clear solution was allowed to evaporate. After a few days, pale yellow-orange crystals of $\mathbf{4}$ separated out from the mother liquid.
2.1.5. Cocrystal 5. 4-Amino-5-chloro-2,6-dimethylpyrimidine $(39 \mathrm{mg})$ and 5-methylthiophene-2-carboxylic acid (35 mg) were dissolved in hot acetonitrile (1:1 molar ratio) and warmed over a water bath for half an hour. The clear solution was allowed to evaporate. After a few days, colourless plate-like crystals of $\mathbf{5}$ separated out from the mother liquid.

2.1.6. Cocrystal 6. 4-Amino-5-chloro-2,6-dimethylpyrimidine $(39 \mathrm{mg})$ and benzoic acid $(31 \mathrm{mg})$ were dissolved in hot methanol (1:1 molar ratio) and warmed over a water bath for half an hour. The clear solution was allowed to evaporate. After a few days, colourless crystals of $\mathbf{6}$ separated out from the mother liquid.

2.1.7. Cocrystal 7. 4-Amino-5-chloro-2,6-dimethylpyrimidine $(39 \mathrm{mg}$ ) and 2-methylbenzoic acid (34 mg) were dissolved in hot ethanol (1:1 molar ratio) and warmed over a water bath for half an hour. The clear solution was allowed to evaporate. After a few days, colourless crystals of $\mathbf{7}$ separated out from the mother liquid.

2.1.8. Cocrystal 8. 4-Amino-5-chloro-2,6-dimethylpyrimidine (39 $\mathrm{mg}$ ) and 3-methylbenzoic acid (34 mg) were dissolved in hot ethanol (1:1 molar ratio) and warmed over a water bath 
for half an hour. The clear solution was allowed to evaporate. After a few days, colourless crystals of $\mathbf{8}$ separated out from the mother liquid.

2.1.9. Cocrystal 9. 4-Amino-5-chloro-2,6-dimethylpyrimidine (39 $\mathrm{mg}$ ) and 4-methylbenzoic acid (34 mg) were dissolved in hot ethanol (1:1 molar ratio) and warmed over a water bath for half an hour. The clear solution was allowed to evaporate. After a few days, colourless crystals of 9 separated out from the mother liquid.

2.1.10. Cocrystal 10. 4-Amino-5-chloro-2,6-dimethylpyrimidine $(39 \mathrm{mg})$ and 4-aminobenzoic acid $(34 \mathrm{mg})$ were dissolved in hot ethanolic solution (1:1 molar ratio) and warmed over a water bath for half an hour. The clear solution was allowed to evaporate. After a few days, colourless crystals of $\mathbf{1 0}$ separated out from the mother liquid.

\subsection{Refinement}

Crystal data, data collection and structure refinement details are summarized in Table 1. For cocrystals $\mathbf{1}, \mathbf{2}$ and $\mathbf{5}$, the $\mathrm{N}-\mathrm{H}$ and $\mathrm{O}-\mathrm{H}$ hydrogens were located in difference Fourier maps and refined isotropically. All other $\mathrm{H}$ atoms were placed in calculated positions and refined using a riding-model approximation, with $\mathrm{C}-\mathrm{H}=0.95(\mathrm{CH})$ or $0.98 \AA\left(\mathrm{CH}_{3}\right)$. Isotropic displacement parameters for these atoms were set at $1.2 U_{\text {eq }}$ (for $\mathrm{CH}$ ) or $1.5 U_{\text {eq }}$ (for $\mathrm{CH}_{3}$ ) of the parent atom. Idealized methyl $\mathrm{H}$ atoms were refined as rotating groups. There are larger than expected residual density peaks close to the $\mathrm{Cl}$ and $\mathrm{S}$ atoms, but these are not chemically sensible and are assumed to be related to the quality of the crystal. For crystals 3,4 and 6-10, the $\mathrm{N}-\mathrm{H}$ and $\mathrm{O}-\mathrm{H}$ hydrogens were located in difference Fourier maps and refined isotropically. All other $\mathrm{H}$ atoms were placed in calculated positions and refined using a riding-model approximation, with $\mathrm{C}-\mathrm{H}=0.95$ (for $\mathrm{H}$ ) or $0.98 \AA$ (for $\mathrm{CH}_{3}$ ). Isotropic displacement parameters for these atoms were set at $1.2 U_{\text {eq }}$ (for $\mathrm{CH}$ ) or $1.5 U_{\text {eq }}$ (for $\mathrm{CH}_{3}$ ) of the parent atom. Idealized methyl $\mathrm{H}$ atoms were refined as rotating groups.

\section{Results and discussion}

The carboxylic acids and 4-amino-5-chloro-2,6-dimethylpyrimidine used in the present study are shown in Schemes 1 and 2. In the crystal structures of 1-10 (except 7), the asymmetric unit contains a molecule of 4-amino-5-chloro-2,6-dimethylpyrimidine and a molecule of the carboxylic acid, whereas in the crystal structure of $\mathbf{7}$, the asymmetric unit contains two molecules of the pyrimidine base and two molecules of the carboxylic acid. Views of the asymmetric units in each of the ten compounds are shown in Figs. 1-10. In all of the ten cocrystals, the carboxyl $\mathrm{H}$ atom was located in a difference Fourier map. The bond lengths and angles of the carboxyl group are similar to those reported for a typical carboxyl group rather than a carboxylate group. In the first series of cocrystals, i.e. 1-4, the carboxyl hydroxy group $(-\mathrm{OH})$ is hydrogen bonded to atom $\mathrm{N} 1(\mathrm{O}-\mathrm{H} \cdots \mathrm{N} 1)$ of the corresponding pyrimidine (single point supramolecular synthon).

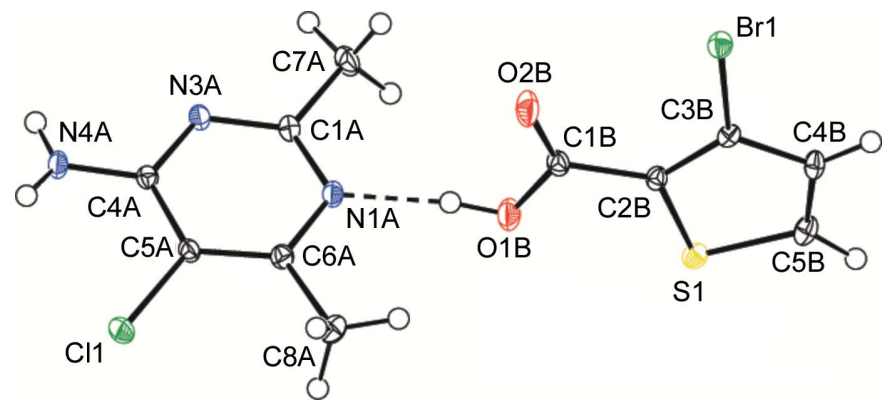

Figure 1

The asymmetric unit of cocrystal $\mathbf{1}$, showing the atom-numbering scheme and displacement ellipsoids drawn at the $30 \%$ probability level. Dashed lines represent hydrogen bonds.

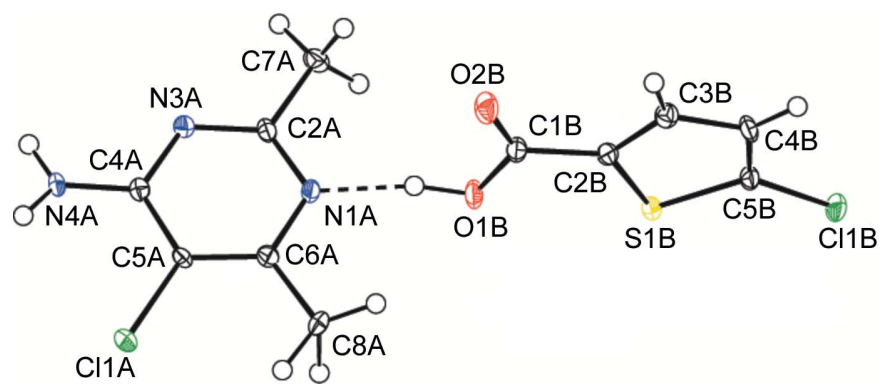

Figure 2

The asymmetric unit of cocrystal $\mathbf{2}$, showing the atom-numbering scheme and displacement ellipsoids drawn at the $30 \%$ probability level. Dashed lines represent hydrogen bonds.

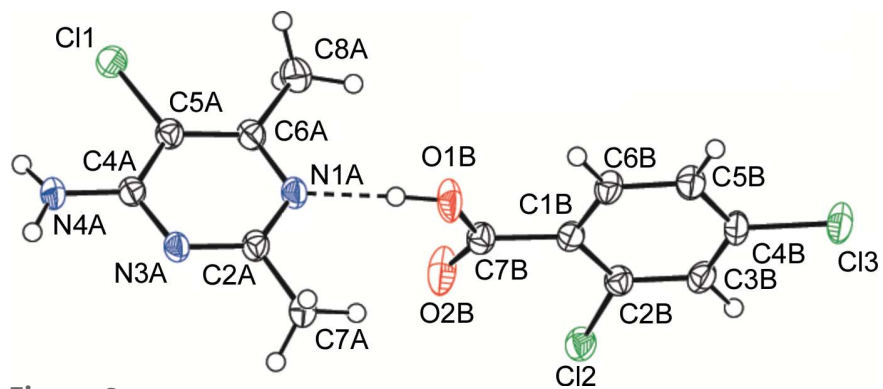

Figure 3

The asymmetric unit of cocrystal $\mathbf{3}$, showing the atom-numbering scheme and displacement ellipsoids drawn at the $30 \%$ probability level. Dashed lines represent hydrogen bonds.

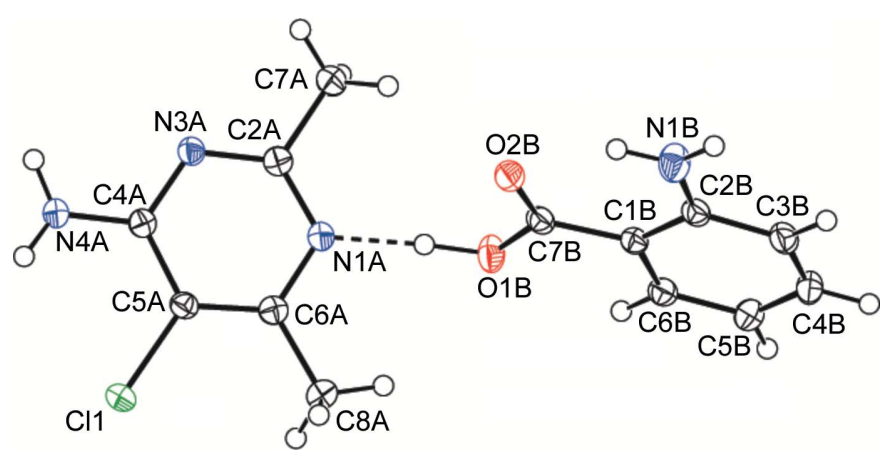

Figure 4

The asymmetric unit of cocrystal $\mathbf{4}$, showing the atom-numbering scheme and displacement ellipsoids drawn at the $30 \%$ probability level. Dashed lines represent hydrogen bonds. 


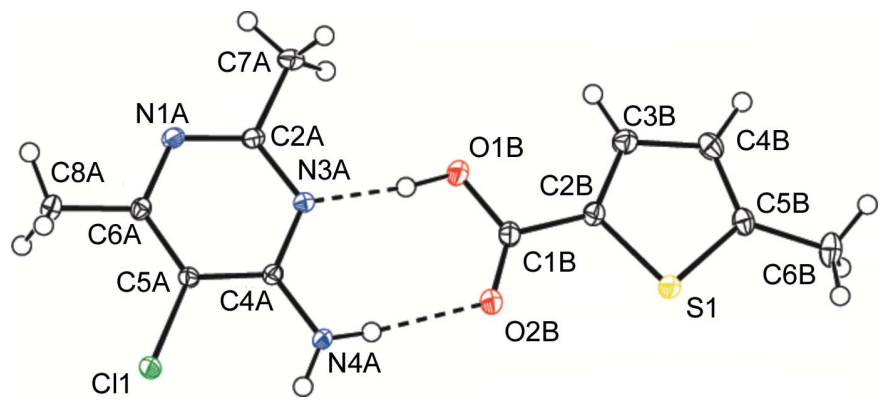

Figure 5

The asymmetric unit of cocrystal $\mathbf{5}$, showing the atom-numbering scheme and displacement ellipsoids drawn at the $30 \%$ probability level. Dashed lines represent hydrogen bonds.

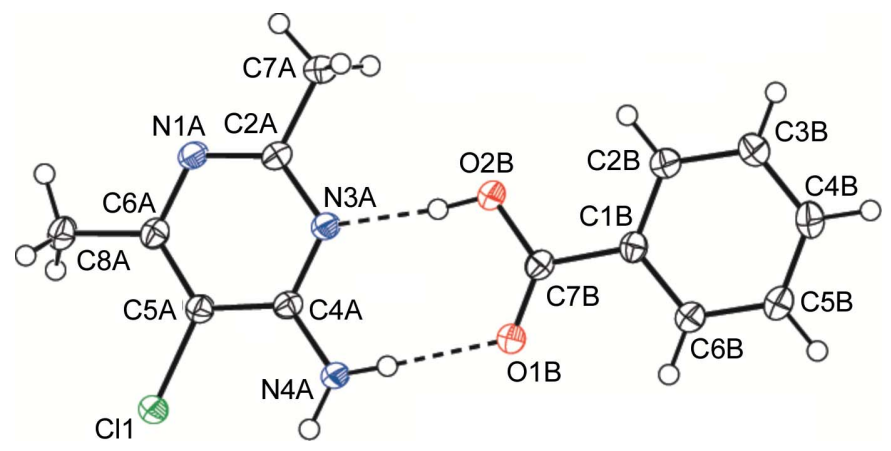

Figure 6

The asymmetric unit of cocrystal $\mathbf{6}$, showing the atom-numbering scheme and displacement ellipsoids drawn at the $30 \%$ probability level. Dashed lines represent hydrogen bonds.

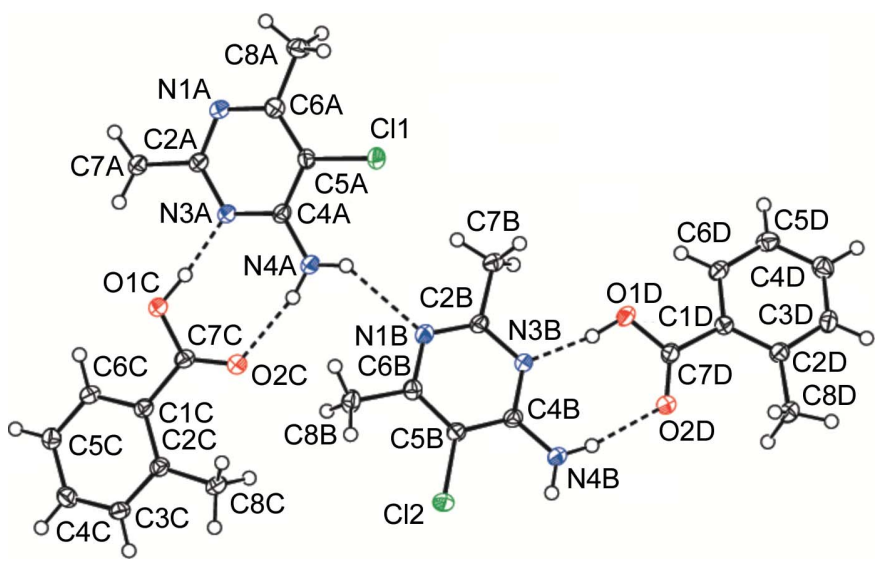

Figure 7

The asymmetric unit of cocrystal $\mathbf{7}$, showing the atom-numbering scheme and displacement ellipsoids drawn at the $30 \%$ probability level. Dashed lines represent hydrogen bonds.

The inversion-related stacked pyrimidines [pyrimidine-pyrimidine stacking interactions with a centroid-to-centroid $(C g 1 \cdots C g 1)$ distance and a slip angle (the angle between the centroid vector and the normal to the plane) of 3.7050 (9) $\AA$ and $21.52^{\circ}$, respectively, for $\mathbf{1}, 3.6228$ (13) $\AA$ and $21.69^{\circ}$ for $\mathbf{2}$, 3.7374 (16) $\AA$ and $24.71^{\circ}$ for 3 , and 3.5182 (9) $\AA$ and $19.73^{\circ}$ for 4] are doubly bridged by the carboxyl groups via $\mathrm{N}-\mathrm{H} \cdots \mathrm{O}$ and $\mathrm{O}-\mathrm{H} \cdots \mathrm{N}$ hydrogen bonds to form a large cage-like

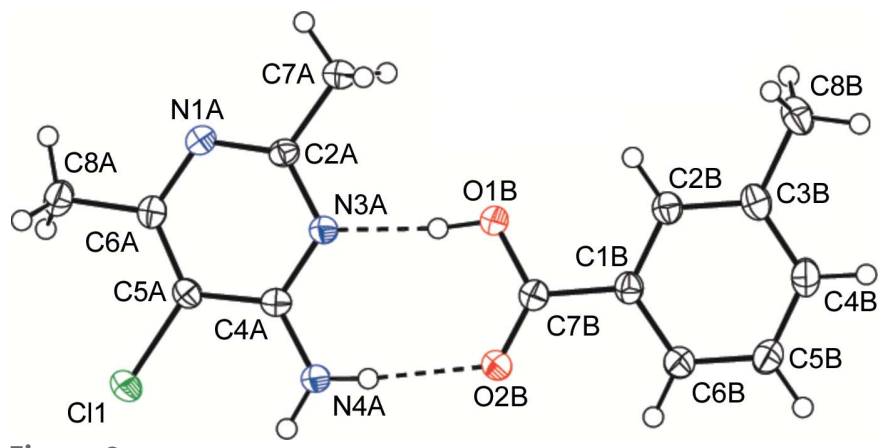

Figure 8

The asymmetric unit of cocrystal $\mathbf{8}$, showing the atom-numbering scheme and displacement ellipsoids drawn at the $30 \%$ probability level. Dashed lines represent hydrogen bonds.

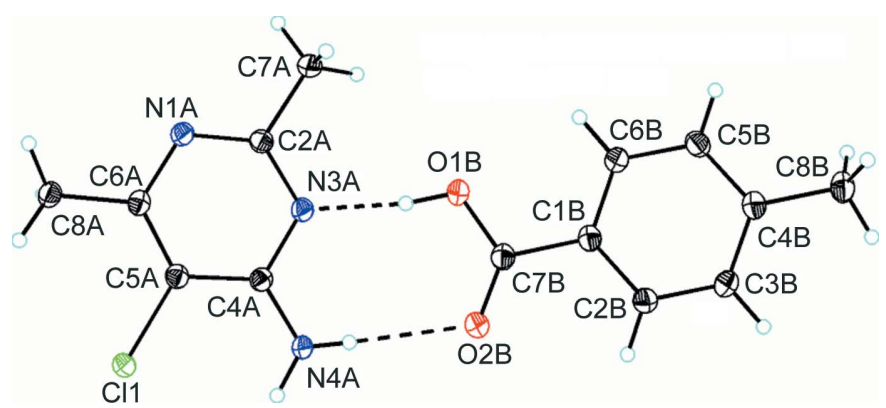

Figure 9

The asymmetric unit of cocrystal $\mathbf{9}$, showing the atom-numbering scheme and displacement ellipsoids drawn at the $30 \%$ probability level. Dashed lines represent hydrogen bonds.

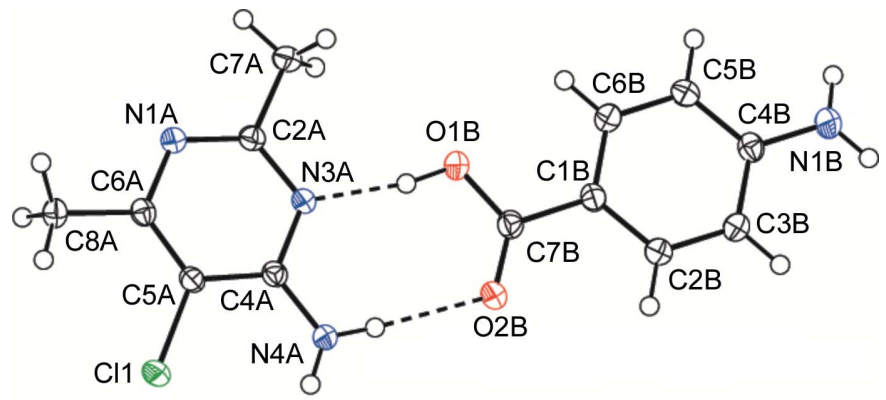

Figure 10

The asymmetric unit of cocrystal 10, showing the atom-numbering scheme and displacement ellipsoids drawn at the $30 \%$ probability level. Dashed lines represent hydrogen bonds.

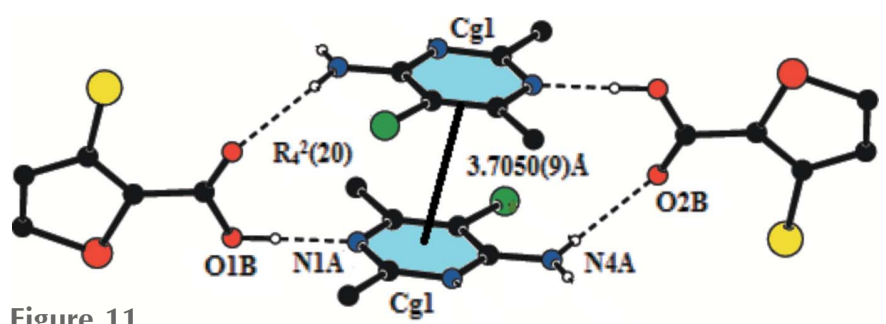

Figure 11

View of the large cage-like tetrameric unit in cocrystal $\mathbf{1}$, with an $R_{4}^{2}(20)$ graph-set ring motif, and the $\pi-\pi$ interactions between nearby pyrimidine moieties ( $C g 1 \cdots C g 1$ centroid-to-centroid interaction; $C g 1$ is the centroid of the $\mathrm{N} 1 A / \mathrm{C} 1 A / \mathrm{N} 3 A / \mathrm{C} 4 A-\mathrm{C} 6 A$ ring). Dashed lined indicate intermolecular $\mathrm{N}-\mathrm{H} \cdots \mathrm{O}$ and $\mathrm{O}-\mathrm{H} \cdots \mathrm{N}$ hydrogen bonds. Generic centroid labels without symmetry codes have been used. 


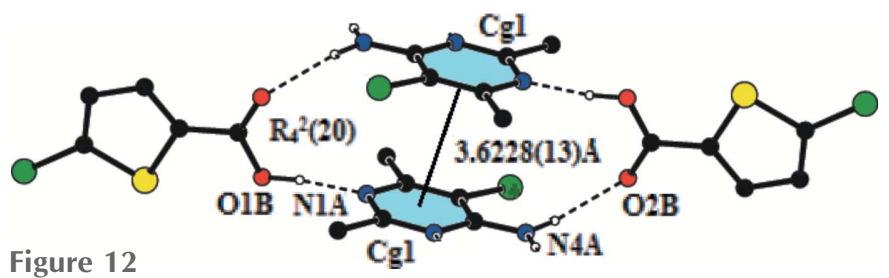

View of the large cage-like tetrameric unit in cocrystal 2, with an $R_{4}^{2}(20)$ graph-set ring motif and $\pi-\pi$ interactions between nearby pyrimidine moieties $(C g 1 \cdots C g 1$ centroid-to-centroid interaction; $C g 1$ is the centroid of the $\mathrm{N} 1 A / \mathrm{C} 1 A / \mathrm{N} 3 A / \mathrm{C} 4 A-\mathrm{C} 6 A$ ring). Dashed lined indicate intermolecular $\mathrm{N}-\mathrm{H} \cdots \mathrm{O}$ and $\mathrm{O}-\mathrm{H} \cdots \mathrm{N}$ hydrogen bonds. Generic centroid labels without symmetry codes have been used.

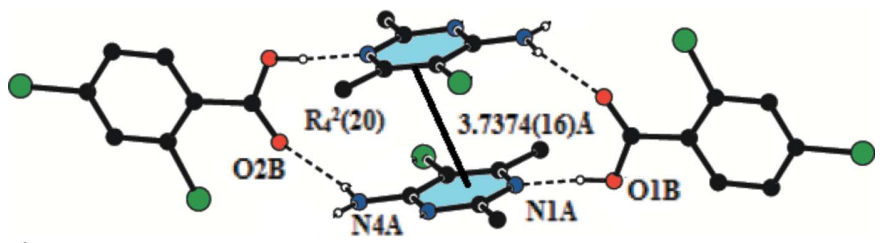

Figure 13

View of the large cage-like tetrameric unit in cocrystal $\mathbf{3}$, with an $R_{4}^{2}(20)$ graph-set ring motif and $\pi-\pi$ interactions between nearby pyrimidine moieties $(C g 1 \cdots C g 1$ centroid-to-centroid interaction; $C g 1$ is the centroid of the $\mathrm{N} 1 A / \mathrm{C} 1 A / \mathrm{N} 3 A / \mathrm{C} 4 A-\mathrm{C} 6 A$ ring). Dashed lined indicate intermolecular $\mathrm{N}-\mathrm{H} \cdots \mathrm{O}$ and $\mathrm{O}-\mathrm{H} \cdots \mathrm{N}$ hydrogen bonds. Generic centroid labels without symmetry codes have been used.

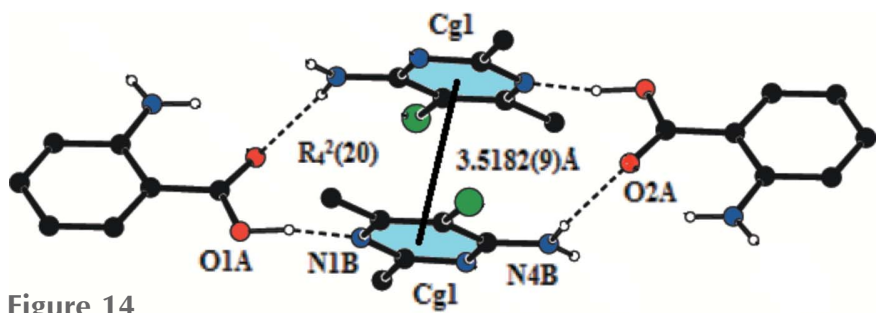

View of the large cage-like tetrameric unit in cocrystal 4, with an $R_{4}^{2}(20)$ graph-set ring motif and $\pi-\pi$ interactions between nearby pyrimidine moieties $(C g 1 \cdots C g 1$ centroid-to-centroid interaction; $C g 1$ is the centroid of the $\mathrm{N} 1 A / \mathrm{C} 1 A / \mathrm{N} 3 A / \mathrm{C} 4 A-\mathrm{C} 6 A$ ring). Dashed lined indicate intermolecular $\mathrm{N}-\mathrm{H} \cdots \mathrm{O}$ and $\mathrm{O}-\mathrm{H} \cdots \mathrm{N}$ hydrogen bonds. Generic centroid labels without symmetry codes have been used.

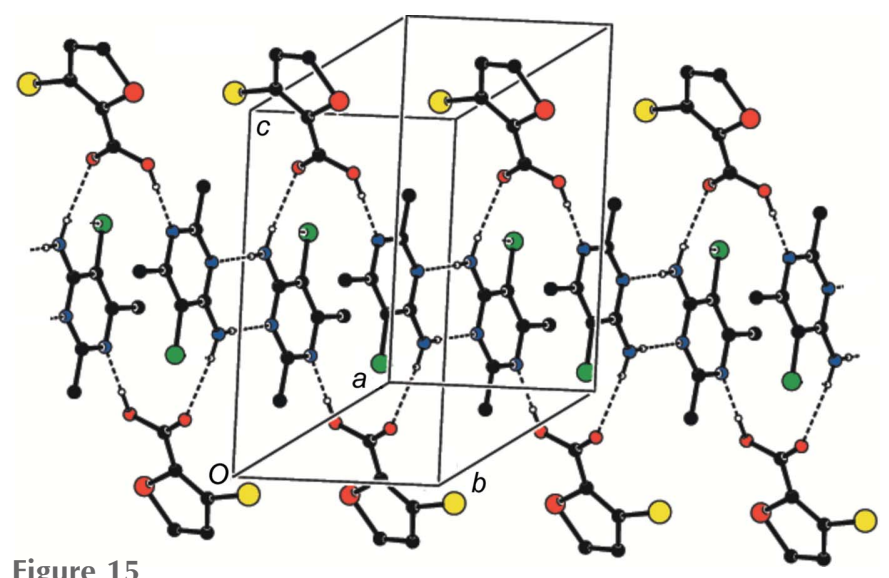

Crystal packing for cocrystal $\mathbf{1}$, viewed along the $a$ axis. Cage-like tetrameric units with $R_{4}^{2}(20)$ graph-set ring motifs are connected via base pairing through a pair of $\mathrm{N}-\mathrm{H} \cdots \mathrm{O}$ and $\mathrm{O}-\mathrm{H} \cdots \mathrm{N}$ hydrogen bonds (dashed lines), generating $R_{2}^{2}(8)$ ring motifs forming chains along $(010) . \mathrm{H}$ atoms not involved in hydrogen bonding have been omitted for clarity.

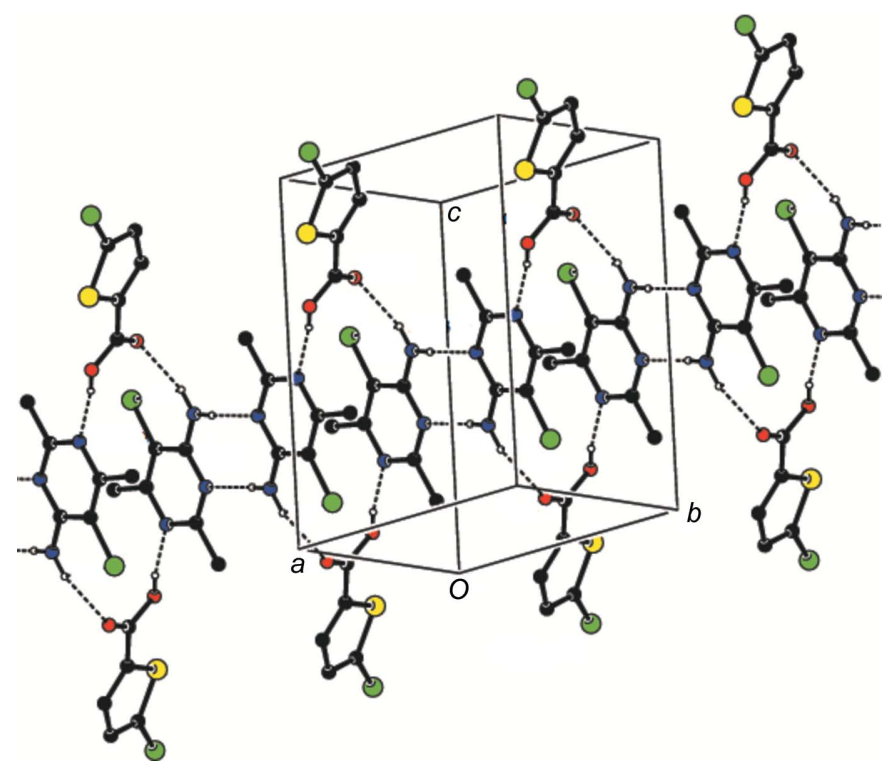

Figure 16

Crystal packing for cocrystal 2, viewed along the $b$ axis. Cage-like tetrameric units with $R_{4}^{2}(20)$ graph-set ring motifs are connected via base pairing through a pair of $\mathrm{N}-\mathrm{H} \cdots \mathrm{O}$ and $\mathrm{O}-\mathrm{H} \cdots \mathrm{N}$ hydrogen bonds (dashed lines), generating $R_{2}^{2}(8)$ ring motifs forming chains along (110). $\mathrm{H}$ atoms not involved in hydrogen bonding have been omitted for clarity.

tetrameric unit with an $R_{4}^{2}(20)$ graph-set ring motif (Figs. 1114). The tetrameric units are further connected via base pairing through a pair of $\mathrm{N}-\mathrm{H} \cdots \mathrm{N}$ hydrogen bonds, generating an $R_{2}^{2}(8)$ motif (supramolecular homosynthon) to form a single block (Figs. 15-18).

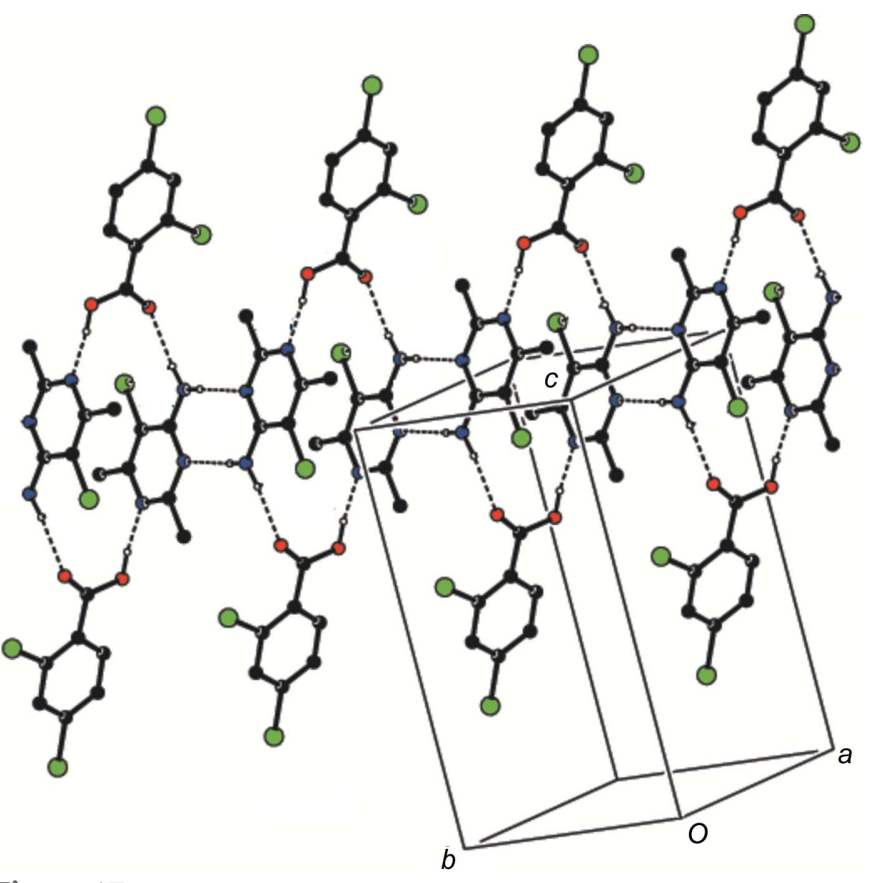

Figure 17

Crystal packing for cocrystal $\mathbf{3}$, viewed along the $a$ axis. Cage-like tetrameric units with $R_{4}^{2}(20)$ graph-set ring motifs are connected via base pairing through a pair of $\mathrm{N}-\mathrm{H} \cdots \mathrm{O}$ and $\mathrm{O}-\mathrm{H} \cdots \mathrm{N}$ hydrogen bonds (dashed lines), generating $R_{2}^{2}(8)$ ring motifs forming chains along (110). $\mathrm{H}$ atoms not involved in hydrogen bonding have been omitted for clarity. 


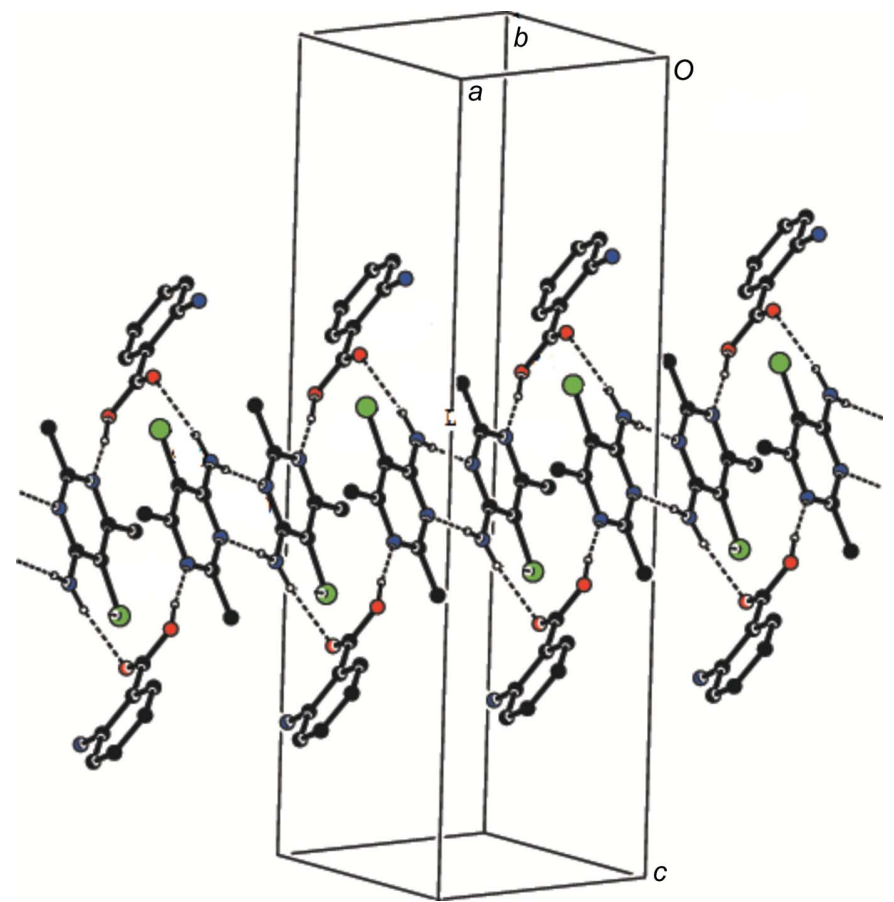

Figure 18

Crystal packing for cocrystal 4, viewed along the $a$ axis. Cage-like tetrameric units with $R_{4}^{2}(20)$ graph-set ring motifs are further connected via base pairing through a pair of $\mathrm{N}-\mathrm{H} \cdots \mathrm{O}$ and $\mathrm{O}-\mathrm{H} \cdots \mathrm{N}$ hydrogen bonds (dashed lines), generating $R_{2}^{2}(8)$ ring motifs forming chains along (110). $\mathrm{H}$ atoms not involved in hydrogen bonding have been omitted for clarity.

In the second series of cocrystals, i.e. 5-10, the carboxyl group interacts with atom $\mathrm{N} 3$ and the adjacent 4 -amino group of the corresponding pyrimidine moiety via $\mathrm{O}-\mathrm{H} \cdots \mathrm{N}$ and $\mathrm{N}-\mathrm{H} \cdots \mathrm{O}$ hydrogen bonds to generate the robust $R_{2}^{2}(8)$ supramolecular heterosynthon. These heterosynthons are further connected by $\mathrm{N}-\mathrm{H} \cdots \mathrm{N}$ hydrogen-bond interactions

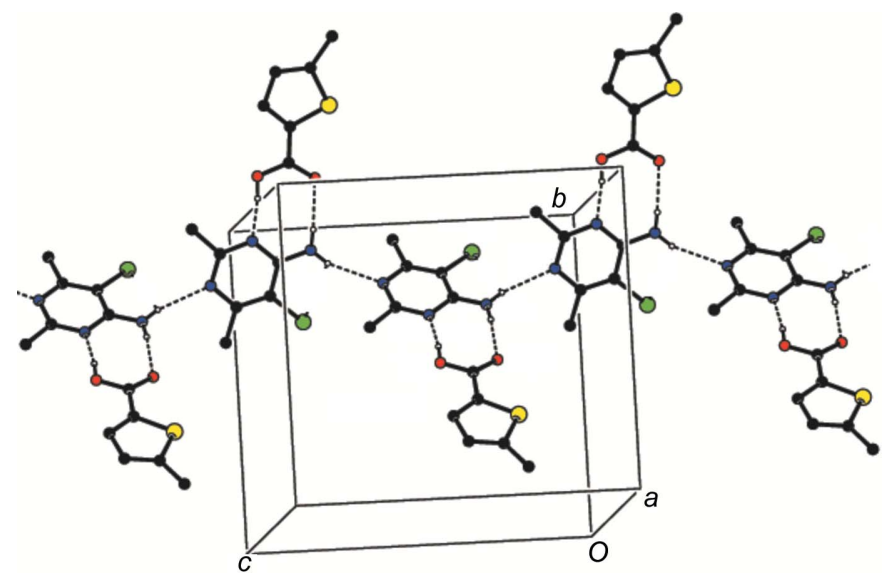

Figure 19

Crystal packing for cocrystal 5, viewed along the $a$ axis. Adjacent heterosynthons are connected by $\mathrm{N}-\mathrm{H} \cdots \mathrm{O}$ and $\mathrm{O}-\mathrm{H} \cdots \mathrm{N}$ hydrogen bonds (dashed lines), forming one-dimensional chains along (001). The heterosynthon occurs through a pair of $\mathrm{N}-\mathrm{H} \cdots \mathrm{N}$ and $\mathrm{O}-\mathrm{H} \cdots \mathrm{N}$ hydrogen bonds (dashed lines), generating $R_{2}^{2}(8)$ ring motifs directed along (010). $\mathrm{H}$ atoms not involved in hydrogen bonding have been omitted for clarity.

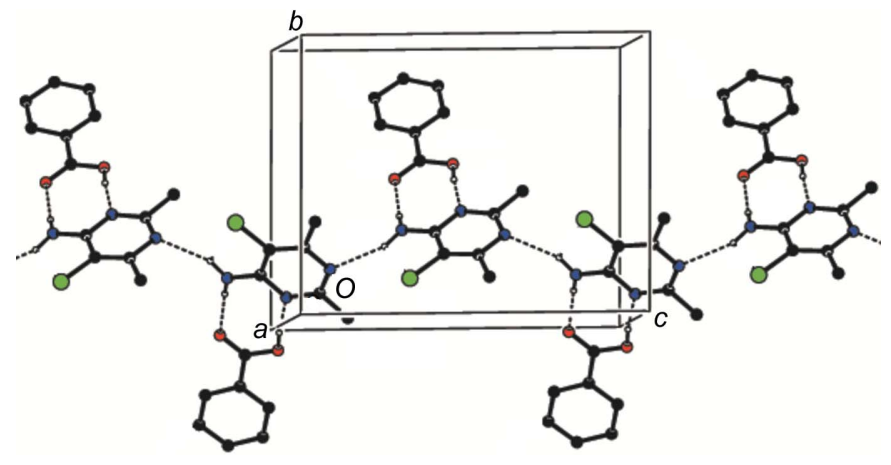

Figure 20

Crystal packing for cocrystal 6, viewed along the $a$ axis. Adjacent heterosynthons are connected by $\mathrm{N}-\mathrm{H} \cdots \mathrm{O}$ hydrogen bonds (dashed lines), forming one-dimensional chains along (001). The heterosynthon occurs through a pair of $\mathrm{N}-\mathrm{H} \cdots \mathrm{N}$ and $\mathrm{O}-\mathrm{H} \cdots \mathrm{N}$ hydrogen bonds (dashed lines), generating $R_{2}^{2}(8)$ ring motifs directed along (010). H atoms not involved in hydrogen bonding have been omitted for clarity.

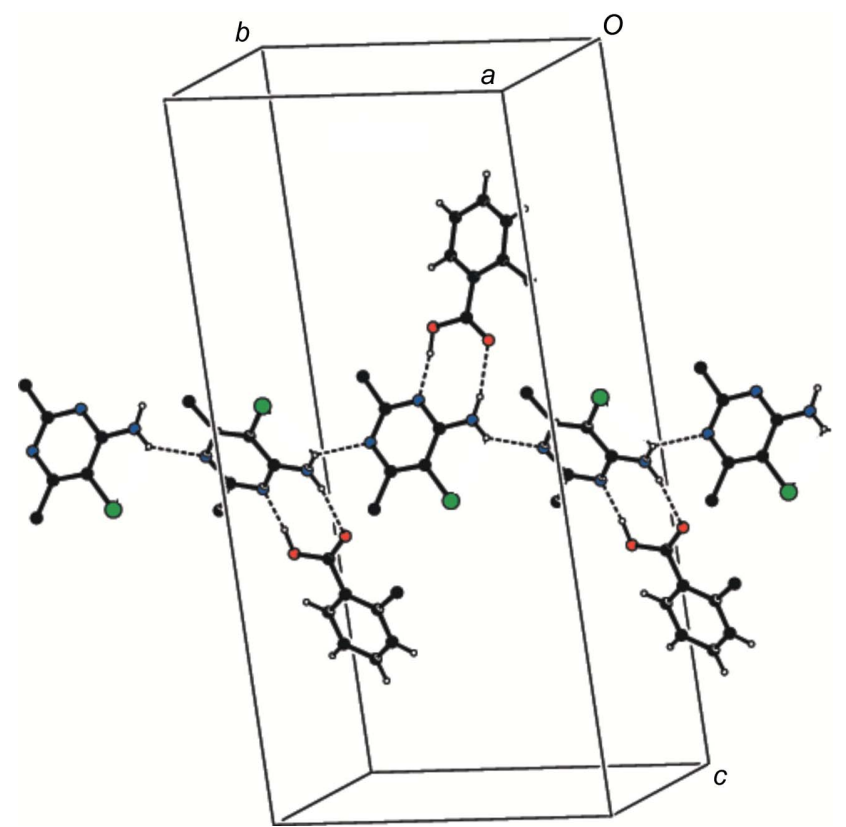

Figure 21

Crystal packing for cocrystal 7, viewed along the $a$ axis. Adjacent heterosynthons are connected by $\mathrm{N}-\mathrm{H} \cdots \mathrm{N}$ hydrogen bonds (dashed lines), forming one-dimensional chains along (001). The heterosynthon occurs through a pair of $\mathrm{N}-\mathrm{H} \cdots \mathrm{O}$ and $\mathrm{O}-\mathrm{H} \cdots \mathrm{N}$ hydrogen bonds (dashed lines), generating $R_{2}^{2}(8)$ ring motifs directed along (010). $\mathrm{H}$ atoms not involved in hydrogen bonding have been omitted for clarity.

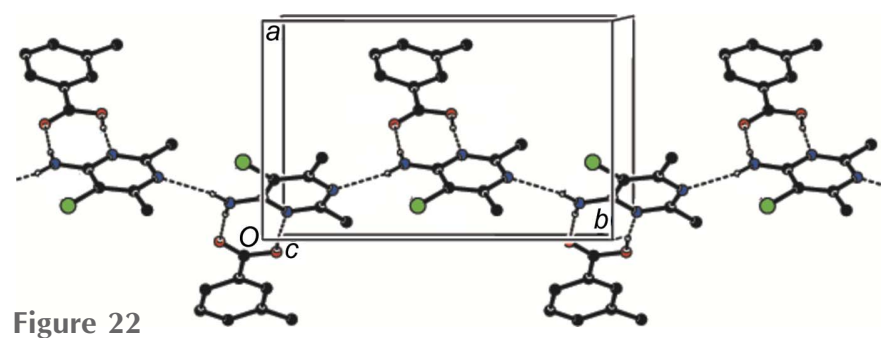

Crystal packing for cocrystal $\mathbf{8}$, viewed along the $c$ axis. Adjacent heterosynthons are connected by $\mathrm{N}-\mathrm{H} \cdots \mathrm{N}$ hydrogen bonds (dashed lines), forming one-dimensional chains along (010). The heterosynthon occurs through a pair of $\mathrm{N}-\mathrm{H} \cdots \mathrm{O}$ and $\mathrm{O}-\mathrm{H} \cdots \mathrm{N}$ hydrogen bonds (dashed lines), generating $R_{2}^{2}(8)$ ring motifs directed along (100). H atoms not involved in hydrogen bonding have been omitted for clarity. 


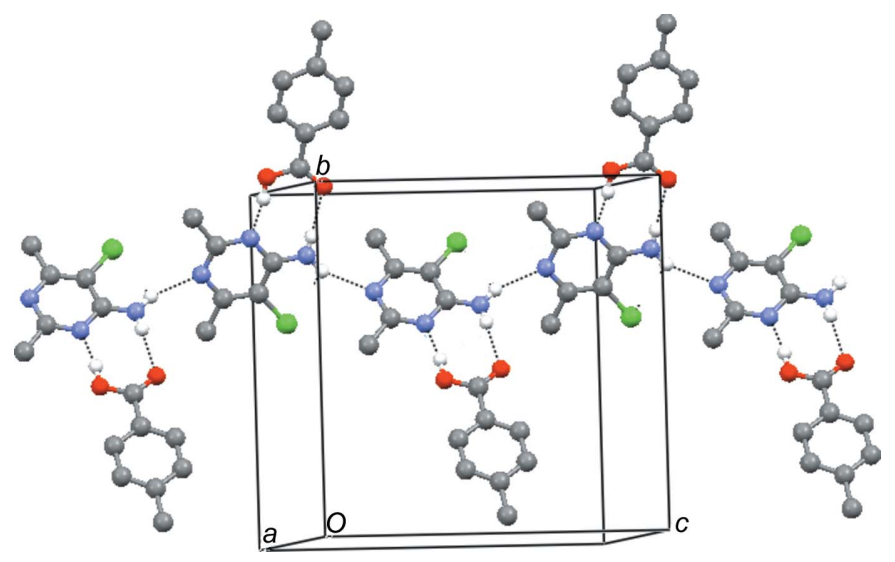

Figure 23

Crystal packing for cocrystal 9, viewed along the $a$ axis. Adjacent heterosynthons are connected by $\mathrm{N}-\mathrm{H} \cdots \mathrm{N}$ hydrogen bonds (dashed lines), forming one-dimensional chains along (001). The heterosynthon occurs through a pair of $\mathrm{N}-\mathrm{H} \cdots \mathrm{O}$ and $\mathrm{O}-\mathrm{H} \cdots \mathrm{N}$ hydrogen bonds (dashed lines), generating $R_{2}^{2}(8)$ ring motifs directed along (010). $\mathrm{H}$ atoms not involved in hydrogen bonding have been omitted for clarity.

in a linear fashion to form a chain-like arrangement (Figs. 1924). The occurrence of two series of cocrystals (depending upon the carboxyl - OH binding with $\mathrm{N} 1$ or N3) is reminiscent of the occurrence of two types of protonation $(\mathrm{N} 1$ or N3) in 6-amino-5-chloro-2,4-dimethylpyrimidin-1-ium 5-chloro-2-hydroxybenzoate, I, and 4-amino-5-chloro-2,6-dimethylpyrimidin-1-ium 5-chloro-2-hydroxybenzoate, II (Rajam et al., 2017).

In the crystal structure of $\mathbf{1}$, neighbouring blocks of the tetrameric units are connected by weak $\mathrm{Br} \cdots \mathrm{Br}$ halogen bonding [3.5693 (3) Aं; Mukherjee \& Desiraju, 2014; Tothadi et al., 2013] and a $\mathrm{C}-\mathrm{H} \cdots \mathrm{Br}$ hydrogen-bond interaction. In addition to this, $\pi-\pi$ stacking interactions are present between the thiophene rings, with an observed interplanar distance of $3.504 \AA$, a centroid-to-centroid $(C g 2 \cdots C g 2 ; C g 2$ is the cen-

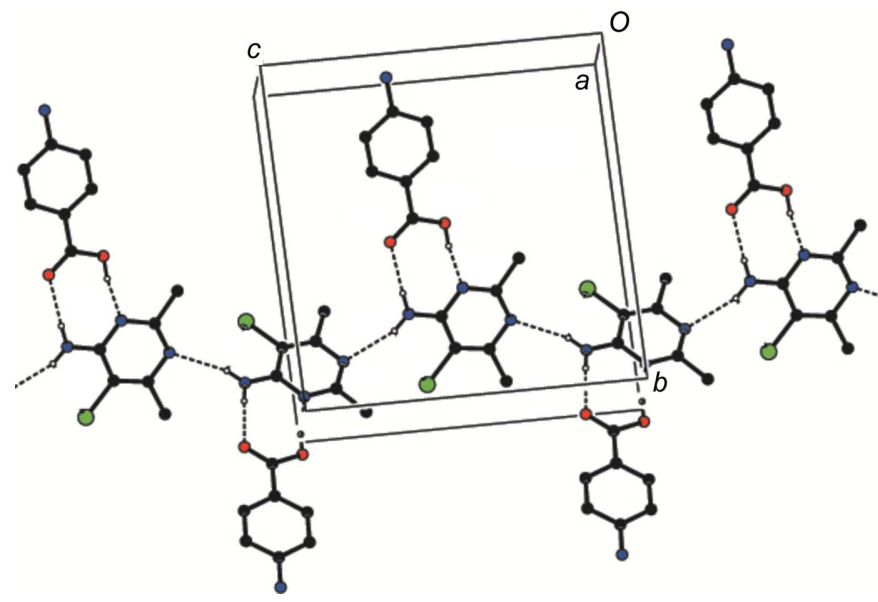

Figure 24

Crystal packing for cocrystal 10, viewed along the $a$ axis. Adjacent heterosynthons are connected by $\mathrm{N}-\mathrm{H} \cdots \mathrm{N}$ hydrogen bonds (dashed lines), forming one-dimensional chains along (001). The heterosynthon occurs through a pair of $\mathrm{N}-\mathrm{H} \cdots \mathrm{O}$ and $\mathrm{O}-\mathrm{H} \cdots \mathrm{N}$ hydrogen bonds (dashed lines), generating $R_{2}^{2}(8)$ ring motifs directed along (010). H atoms not involved in hydrogen bonding have been omitted for clarity.

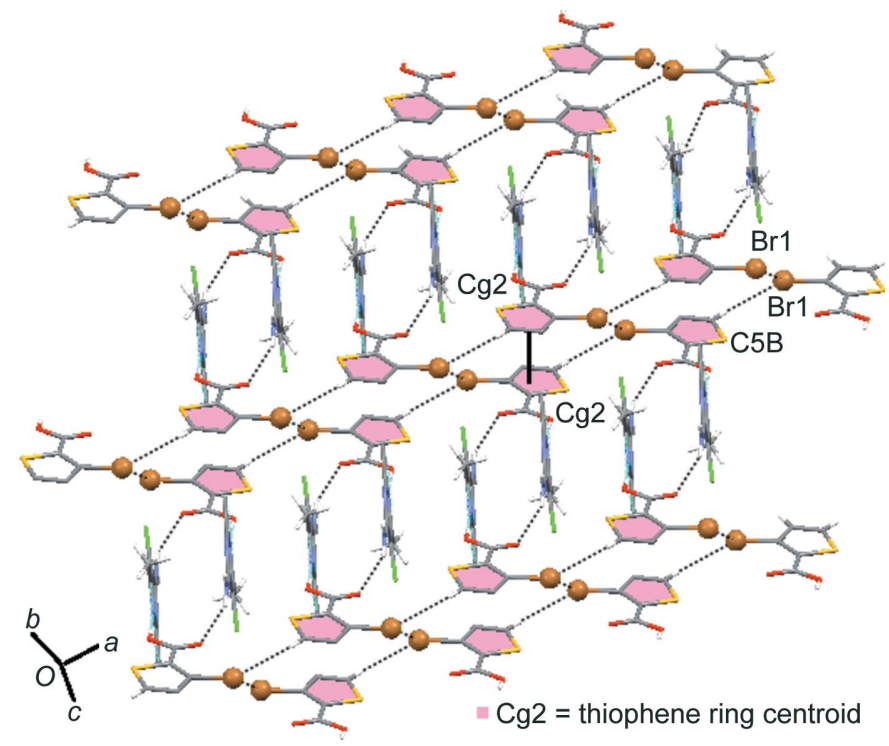

Figure 25

An overall packing view of cocrystal $\mathbf{1}$ containing large cage-like tetrameric units with $R_{4}^{2}(20)$ graph-set ring motifs derived from intermolecular $\mathrm{N}-\mathrm{H} \cdots \mathrm{O}$ and $\mathrm{O}-\mathrm{H} \cdots \mathrm{N}$ hydrogen bonds (dashed lines) and $\pi-\pi$ interactions between nearby pyrimidine moieties. Neighbouring blocks are connected by weak $\mathrm{Br}($ acid $) \cdots \mathrm{Br}($ acid $), \mathrm{C}-\mathrm{H} \cdots \mathrm{Br}$ and $\pi($ acid $)-\pi$ (acid) $(C g 2 \cdots C g 2)$ stacking of thiophene (pink) moieties. $\mathrm{H}$ atoms not involved in hydrogen bonding have been omitted for clarity. Generic atom and centroid labels without symmetry codes are shown

troid of the $\mathrm{S} 1 B / \mathrm{C} 2 B-\mathrm{C} 5 B$ ring) distance of 3.6133 (10) $\AA$ and a slip angle (the angle between the centroid vector and the normal to the plane) of $14.15^{\circ}$, stabilizing the crystal structure and forming a three-dimensional superstructure (Fig. 25).

For the crystal structure of $\mathbf{2}$, neighbouring blocks of the tetrameric units are connected by weak $\mathrm{Cl}$. C Cl halogen bonds [3.3736 (9) §; Mukherjee \& Desiraju, 2014; Tothadi et al., 2013] and $\mathrm{C}-\mathrm{H} \cdots \mathrm{Cl}$ hydrogen-bond interactions. In addition to this, $\pi-\pi$ interactions are observed between the thiophene rings, with an observed interplanar distance of $3.493 \AA$, a centroid-to-centroid $(\mathrm{Cg} 2 \cdots \mathrm{Cg} 2 ; \mathrm{Cg} 2$ is the centroid of the $\mathrm{S} 1 B / \mathrm{C} 2 B-\mathrm{C} 5 B$ ring) distance of 3.8466 (14) $\AA$ and a slip angle of $24.77^{\circ}$ (Fig. 26).

In the crystal structure of $\mathbf{3}$, neighbouring blocks of the tetrameric units are connected by a $\mathrm{Cl} \cdots \mathrm{Cl}$ halogen bond [3.4967 (9) Аं; Mukherjee \& Desiraju, 2014; Tothadi et al., 2013]. In addition to this, $\pi-\pi$ stacking interactions between the benzene rings, with an observed interplanar distance of $3.477 \AA$, a centroid-to-centroid $(C g 2 \cdots C g 2 ; C g 2$ is the centroid of the $\mathrm{C} 1 B-\mathrm{C} 6 B$ ring) distance of 3.8815 (17) $\AA$ and a slip angle of $26.39^{\circ}$, are also present to stabilize the supramolecular architecture (Fig. 27).

In the crystal structure of $\mathbf{4}$, neighbouring blocks of the tetrameric units are connected by aromatic $\mathrm{C}-\mathrm{H} \cdots \pi$ interactions, with the distance from the proton to the acceptor ring, i.e. $(\mathrm{C} 4 B-) \mathrm{H} 4 B A \cdots C g 2$, being $2.66 \AA(C g 2$ is the centroid of the $\mathrm{C} 1 B-\mathrm{C} 6 B$ ring), forming a three-dimensional superstructure (Fig. 28).

In the crystal structure of $\mathbf{5}$, two inversion-related heterotetramers are connected through $\mathrm{N}-\mathrm{H} \cdots \mathrm{N}$ hydrogen bonds 


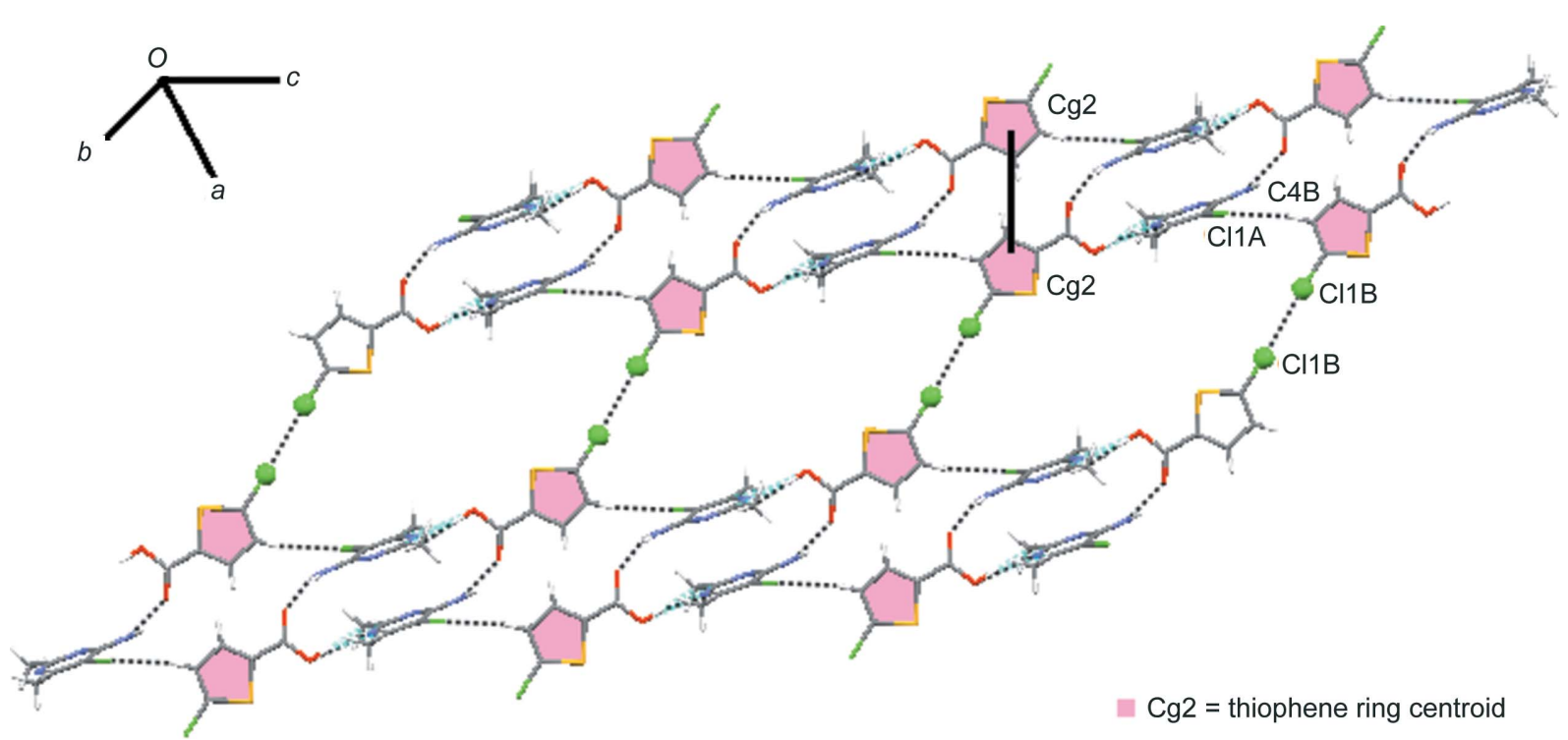

Figure 26

An overall packing view of cocrystal 2 containing large cage-like tetrameric units with $R_{4}^{2}(20)$ graph-set ring motifs derived from intermolecular $\mathrm{N}-$ $\mathrm{H} \cdots \mathrm{O}$ and $\mathrm{O}-\mathrm{H} \cdots \mathrm{N}$ hydrogen bonds (dashed lines) and $\pi-\pi$ interactions between nearby pyrimidine moieties. Neighbouring blocks are connected by weak $\mathrm{Cl}($ acid $) \cdots \mathrm{Cl}($ acid $), \mathrm{C}-\mathrm{H} \cdots \mathrm{Cl}$ and $\pi$ (acid) $-\pi($ acid $)(C g 2 \cdots C g 2)$ stacking of thiophene (pink) moieties. $\mathrm{H}$ atoms not involved in hydrogen bonding have been omitted for clarity. Generic atom and centroid labels without symmetry codes are shown

and a Cl . O halogen bond (3.2022 ^; Mukherjee \& Desiraju, 2014; Lu et al., 2009) to form a cyclic ring motif. In addition to this, $\pi-\pi$ stacking interactions between the pyrimidine and the acid ring, with an observed interplanar distance of $3.520 \AA$, a centroid-to-centroid $(C g 1 \cdots C g 2 ; C g 1$ is the centroid of the $\mathrm{N} 1 A / \mathrm{C} 2 A / \mathrm{N} 3 A / \mathrm{C} 4 A-\mathrm{C} 6 A$ ring and $C g 2$ is the centroid of the $\mathrm{S} 1 B / \mathrm{C} 1 B-\mathrm{C} 5 B$ ring) distance of 3.8002 (16) $\AA$ and a slip angle of $21.06^{\circ}$, are also present to stabilize the crystal structure (Fig. 29).

In the crystal structure of $\mathbf{6}$, the hydrogen-bonded ring is formed by the interconnection of two inversion-related heterotetramers through $\mathrm{N}-\mathrm{H} \cdots \mathrm{N}$ hydrogen bonds and a
Cl . O halogen bond (2.9926 ̊; Mukherjee \& Desiraju, 2014; Lu et al., 2009) (Fig. 30).

In the crystal structure of 7, two of the heterotetramers are connected through $\mathrm{N}-\mathrm{H} \cdots \mathrm{N}$ hydrogen bonds and a $\mathrm{Cl} \cdots \mathrm{O}$ halogen bond [3.121 (18) A; Mukherjee \& Desiraju, 2014; Lu et al., 2009] to form a cyclic ring. Two distinct $\pi-\pi$ stacking interactions between pyrimidine/pyrimidine [with an observed interplanar distance of $3.429 \AA$, a centroid-to-centroid $(C g 1 \cdots C g 2 ; C g 1$ is the centroid of the $\mathrm{N} 1 A / \mathrm{C} 2 A / \mathrm{N} 3 A / \mathrm{C} 4 A-$ $\mathrm{C} 6 A$ ring and $C g 2$ is the centroid of the $\mathrm{N} 1 B / \mathrm{C} 2 B / \mathrm{N} 3 B / \mathrm{C} 4 B-$ C6 $B$ ring) distance of 3.6946 (15) $\AA$ and slip angle of $21.87^{\circ}$ ] and acid/acid [with an observed interplanar distance of

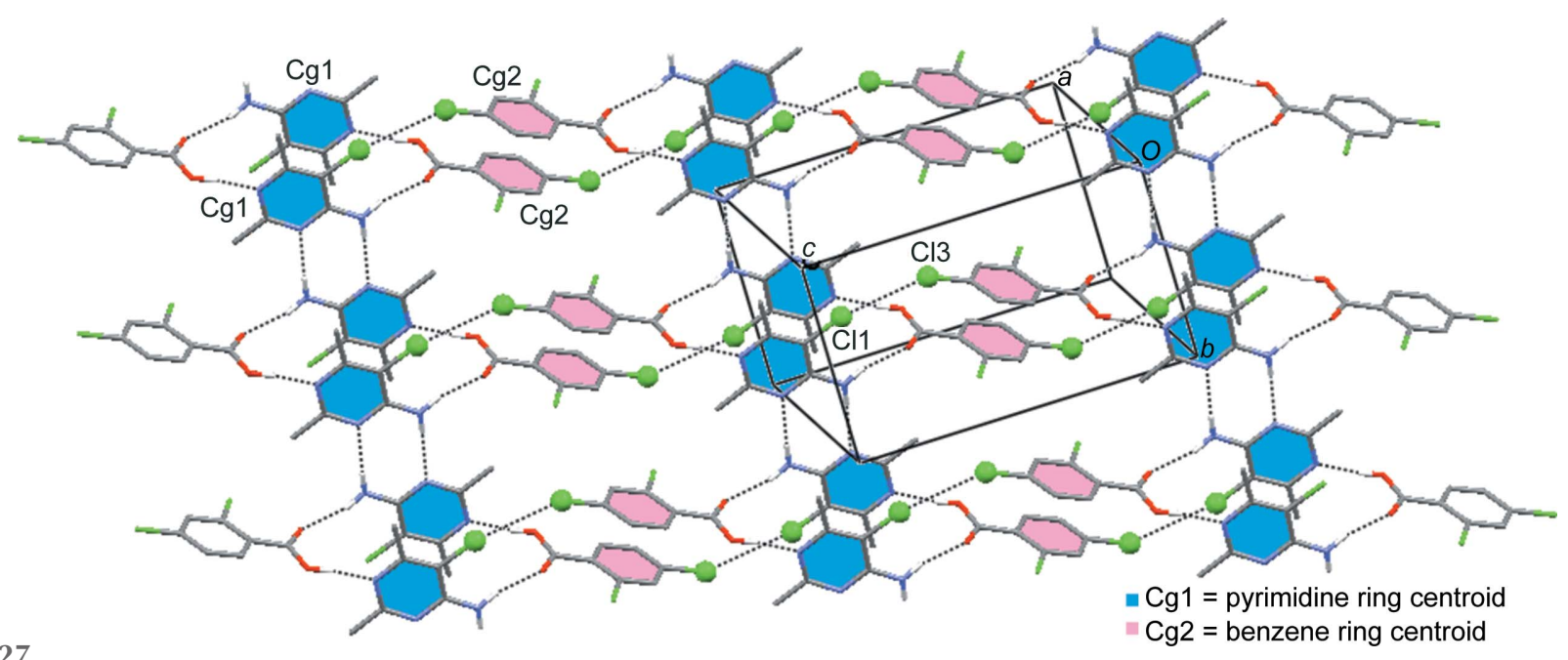

Figure 27

An overall packing view of cocrystal 3 containing large cage-like tetrameric units with $R_{4}^{2}(20)$ graph-set ring motifs derived from intermolecular N$\mathrm{H} \cdots \mathrm{O}$ and $\mathrm{O}-\mathrm{H} \cdots \mathrm{N}$ hydrogen bonds (dashed lines) and $\pi-\pi$ interactions, i.e. $C g 1 \cdots C g 1$ centroid-to-centroid interactions (blue), of nearby pyrimidine moieties. Neighbouring blocks are further connected by weak $\mathrm{Cl}$ (pyrimidine) $\cdots \mathrm{Cl}($ acid $), \mathrm{C}-\mathrm{H} \cdots \mathrm{Cl}$ and $\pi($ acid $)-\pi($ acid $)(C g 2 \cdots C g 2)$ stacking of phenyl (pink) moieties. H atoms not involved in hydrogen bonding have been omitted for clarity. Generic atom and centroid labels without symmetry codes are shown 


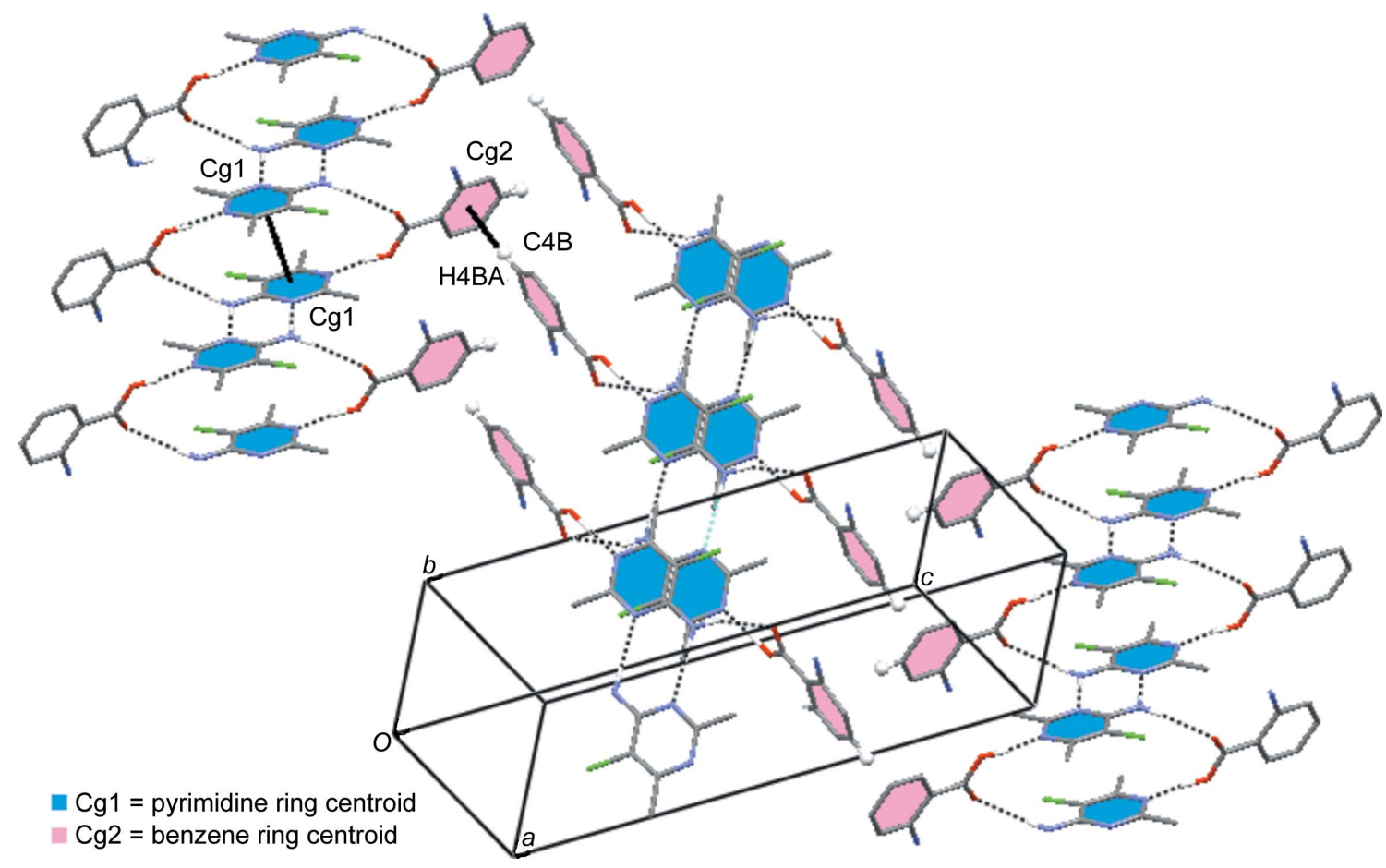

Figure 28

An overall packing view of cocrystal 4 containing large cage-like tetrameric units with $R_{4}^{2}(20)$ graph-set ring motifs derived from intermolecular N$\mathrm{H} \cdots \mathrm{O}$ and $\mathrm{O}-\mathrm{H} \cdots \mathrm{N}$ hydrogen bonds (dashed lines) and $\pi-\pi$ interactions, i.e. $C g 1 \cdots C g 1$ centroid-to-centroid interactions (blue), of nearby pyrimidine moieties. Neighbouring blocks are connected by weak $C g 2 \cdots \pi$ interactions $(\mathrm{C}-\mathrm{H} \cdots C g 2$, pink) of nearby phenyl moieties (dashed lines) contribute to form a three-dimensional superstructure. $\mathrm{H}$ atoms not involved in hydrogen bonding have been omitted for clarity. Generic atom and centroid labels without symmetry codes are shown

$3.353 \AA$, a centroid-to-centroid $(C g 3 \cdots C g 4 ; C g 3$ is the centroid of the $\mathrm{C} 1 \mathrm{C}-\mathrm{C} 6 \mathrm{C}$ ring and $\mathrm{Cg} 4$ is the centroid of the $\mathrm{C} 1 D-\mathrm{C} 6 D$ ring) distance of 3.6319 (17) $\AA$ and a slip angle of $22.61^{\circ}$ ] are also observed (Fig. 31).

In the crystal structure of $\mathbf{8}$, two of the inversion-related heterotetramers are connected through $\mathrm{N}-\mathrm{H} \cdots \mathrm{N}$ and $\mathrm{C}-$

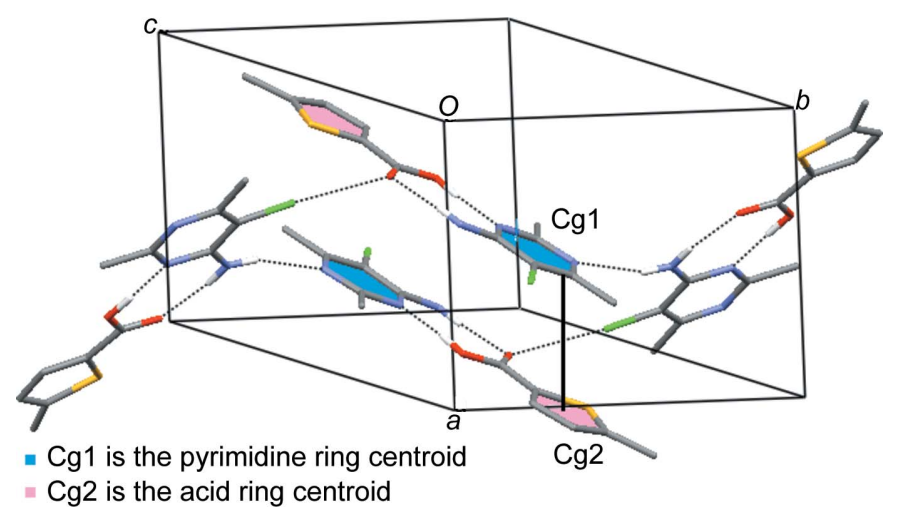

Figure 29

An overall packing view of cocrystal $\mathbf{5}$, viewed along the $c$ axis, containing large cage-like hydrogen bonded units with $R_{4}^{4}(24)$ graph-set ring motifs derived from intermolecular $\mathrm{N}-\mathrm{H} \cdots \mathrm{N}$ and $\mathrm{O}-\mathrm{H} \cdots \mathrm{N}$ hydrogen bonds (dashed lines) connecting the pyrimidine (blue) and thiophene (pink) rings and $\mathrm{C}-\mathrm{Cl} \cdots \mathrm{O}$ intermolecular interactions which are displayed. Additional weak $\pi-\pi C g 1$ (pyrimidine) .. $C g 2$ (thiophene) intermolecular interactions are also present forming a two-dimensional network along (001). $\mathrm{H}$ atoms not involved in hydrogen bonding have been omitted for clarity
$\mathrm{H}$. . O hydrogen-bond interactions to form a cyclic ring motif. $\pi-\pi$ stacking interactions between the pyrimidine rings, with an observed interplanar distance of $3.508 \AA$, a centroid-tocentroid $(C g 1 \cdots C g 1 ; C g 1$ is the centroid of the $\mathrm{N} 1 A / \mathrm{C} 2 A$ /

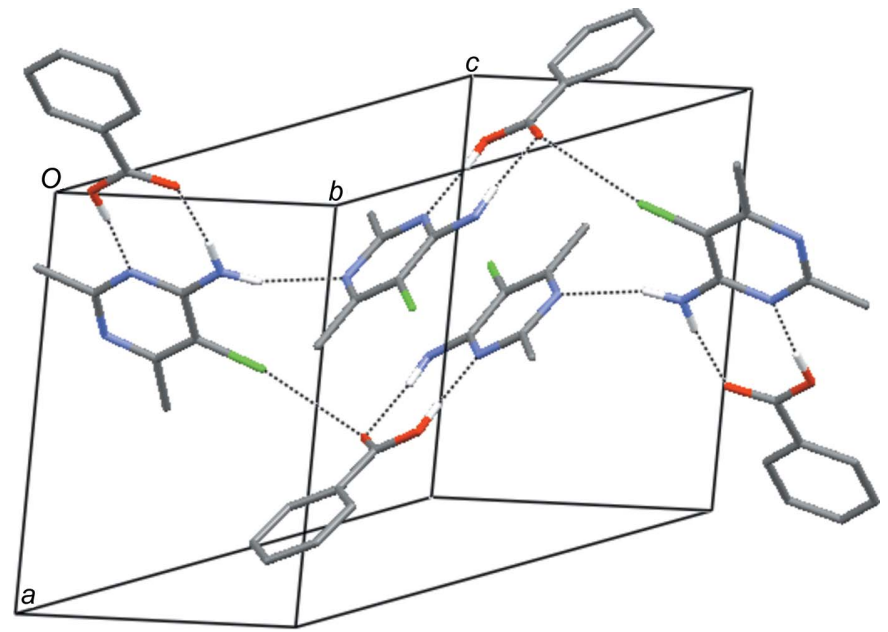

Figure 30

An overall packing view of cocrystal 6, viewed along the $c$ axis, containing large cage-like hydrogen bonded units with $R_{4}^{4}(24)$ graph-set ring motifs derived from intermolecular $\mathrm{N}-\mathrm{H} \cdots \mathrm{N}$ and $\mathrm{O}-\mathrm{H} \cdots \mathrm{N}$ hydrogen bonds (dashed lines) connecting the pyrimidine and phenyl rings and $\mathrm{C}-\mathrm{Cl} \cdots \mathrm{O}$ intermolecular interactions which are displayed. Additional weak $\pi-\pi$ Cg1(pyrimidine) $\cdots C$ Cg2(phenyl) intermolecular interactions (not indicated) are also present forming a two-dimensional network along (001). $\mathrm{H}$ atoms not involved in hydrogen bonding have been omitted for clarity 


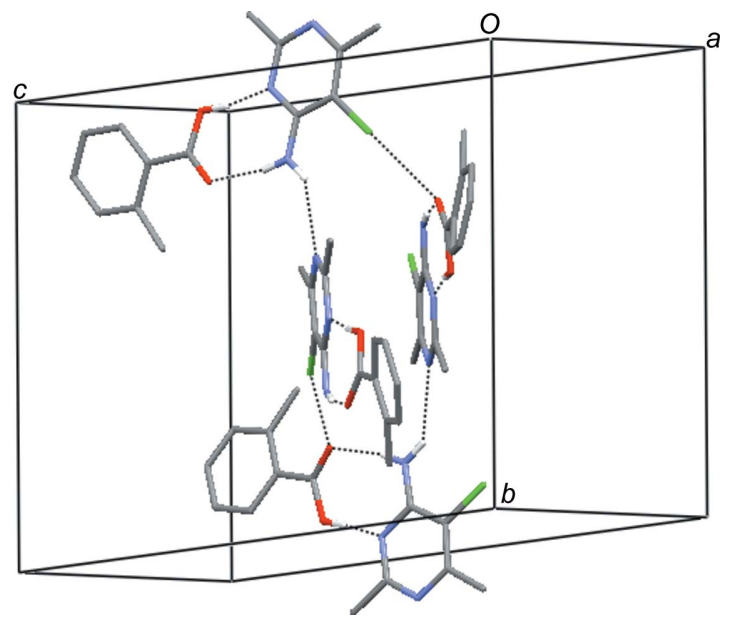

Figure 31

An overall packing view of cocrystal 7, viewed along the $c$ axis containing large cage-like hydrogen bonded units with $R_{4}^{4}(20)$ graph-set ring motifs derived from intermolecular $\mathrm{N}-\mathrm{H} \cdots \mathrm{N}$ and $\mathrm{O}-\mathrm{H} \cdots \mathrm{N}$ hydrogen bonds (dashed lines) connecting the pyrimidine and phenyl rings and $\mathrm{C}-\mathrm{Cl} \cdots \mathrm{O}$ intermolecular interactions which are displayed. Additional weak $\pi-\pi$ $C g$ (pyrimidine) $\cdots C g 2$ (phenyl) intermolecular interactions (not indicated) are also present forming a two-dimensional network along (001). $\mathrm{H}$ atoms not involved in hydrogen bonding have been omitted for clarity

$\mathrm{N} 3 A / \mathrm{C} 4 A-\mathrm{C} 6 A$ ring) distance of 3.6145 (7) $\AA$ and a slip angle of $13.91^{\circ}$, are also observed (Fig. 32).

In the crystal structure of $\mathbf{9}$, two inversion-related heterotetramers are connected through $\mathrm{N}-\mathrm{H} \cdots \mathrm{N}$ and $\mathrm{C}-\mathrm{H} \cdots \mathrm{O}$ hydrogen-bond interactions to form a cyclic ring motif. In addition to this, $\pi-\pi$ stacking interactions between the pyrimidine moiety and the acid ring, with an observed interplanar distance of $3.384 \AA$, a centroid-to-centroid $(C g 1 \cdots C g 2 ; C g 1$ is the centroid of the $\mathrm{N} 1 A / \mathrm{C} 2 A / \mathrm{N} 3 A / \mathrm{C} 4 A-\mathrm{C} 6 A$ ring and $C g 2$ is the centroid of the $\mathrm{C} 1 B-\mathrm{C} 6 B$ ring) distance of $3.6186 \AA$ and a slip angle of $18.94^{\circ}$ are also present to help stabilize the crystal structure (Fig. 33).

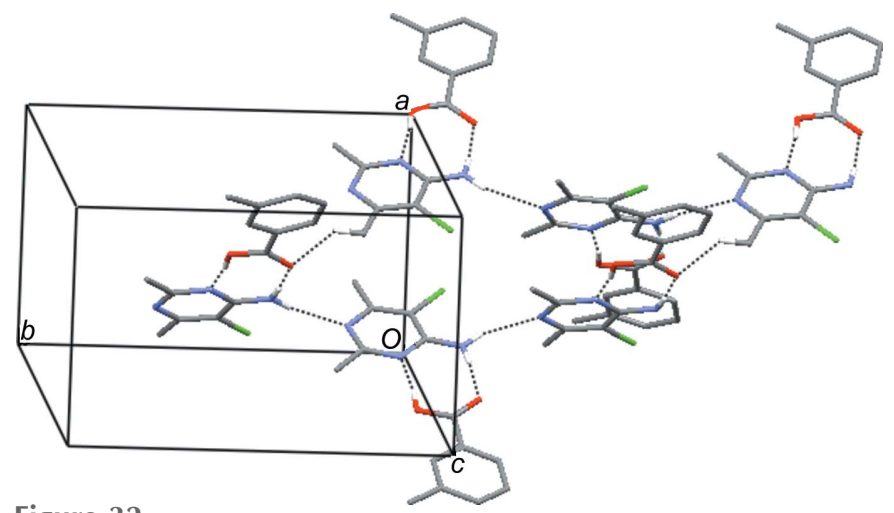

Figure 32

An overall packing view of cocrystal $\mathbf{8}$, viewed along the $c$ axis, containing large cage-like hydrogen-bonded units with $R_{8}^{8}(34)$ graph-set ring motifs derived from intermolecular $\mathrm{N}-\mathrm{H} \cdots \mathrm{N}$ and $\mathrm{O}-\mathrm{H} \cdots \mathrm{N}$ hydrogen bonds (dashed lines) connecting the pyrimidine and phenyl rings and $\mathrm{N}-\mathrm{H} \cdots \mathrm{N}$ intermolecular interactions which are displayed. Additional weak $\pi-\pi$ Cg1(pyrimidine)...Cg1(pyrimidine) intermolecular interactions (not indicated) are also present forming a two-dimensional network along (010). $\mathrm{H}$ atoms not involved in hydrogen bonding have been omitted for clarity

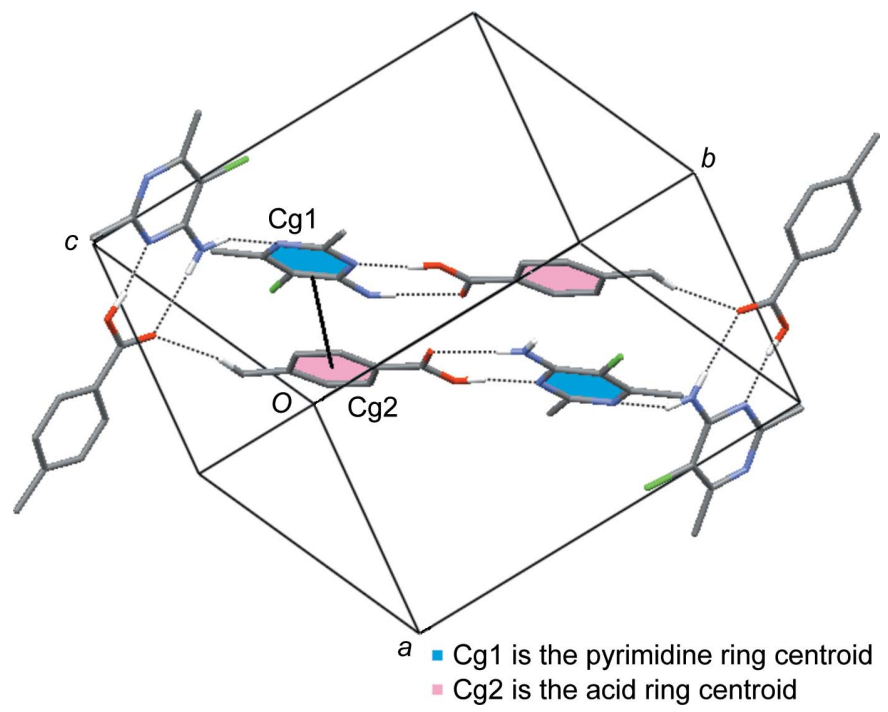

Figure 33

An overall packing view of cocrystal 9, viewed along the $c$ axis containing large cage-like hydrogen bonded units with $R_{8}^{8}(34)$ graph-set ring motifs derived from intermolecular $\mathrm{N}-\mathrm{H} \cdots \mathrm{N}$ and $\mathrm{O}-\mathrm{H} \cdots \mathrm{N}$ hydrogen bonds (dashed lines) connecting the pyrimidine and phenyl rings and $\mathrm{N}-\mathrm{H} \cdots \mathrm{N}$ intermolecular interactions which are displayed. Additional weak $\pi-\pi$ $C g 1$ (pyrimidine) $\cdots C g$ 2(phenyl) intermolecular interactions are also present forming a two-dimensional network along (111). H atoms not involved in hydrogen bonding have been omitted for clarity

In the crystal structure of $\mathbf{1 0}$, two of the inversion-related heterotetramers are connected through $\mathrm{N}-\mathrm{H} \cdots \mathrm{N}$ and $\mathrm{N}-$ $\mathrm{H}$... O hydrogen-bond interactions to form a cyclic ring motif. In addition to this, $\pi-\pi$ stacking interactions between the rings of the pyrimidine and acid molecules are observed, with an

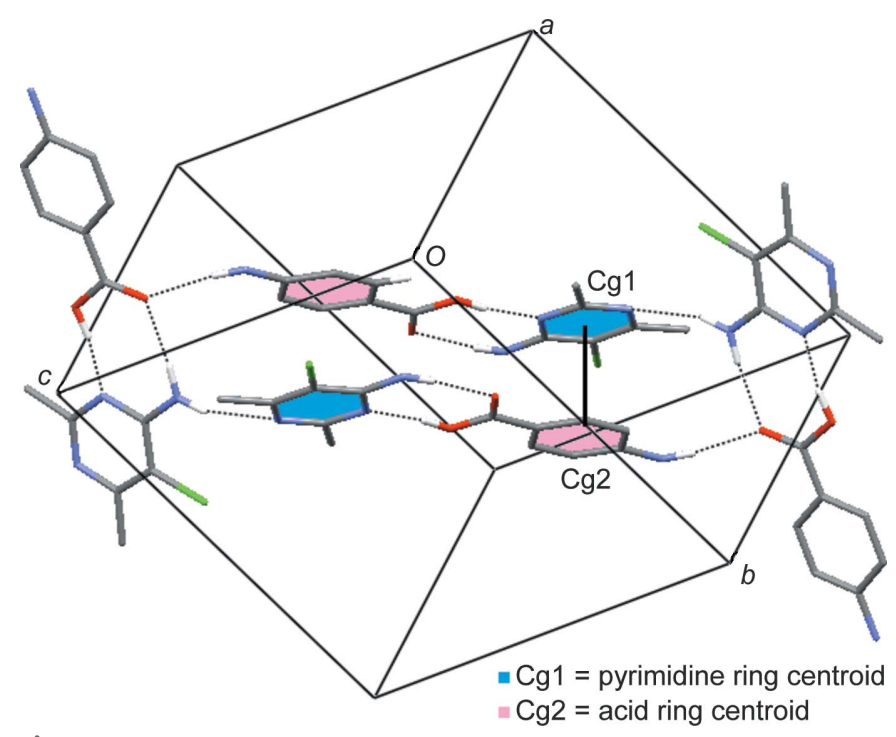

Figure 34

An overall packing view of cocrystal 10, viewed along the $c$ axis containing large cage-like hydrogen-bonded units with $R_{8}^{8}(34)$ graph-set ring motifs derived from intermolecular $\mathrm{N}-\mathrm{H} \cdots \mathrm{N}$ and $\mathrm{O}-\mathrm{H} \cdots \mathrm{N}$ hydrogen bonds (dashed lines) connecting the pyrimidine and phenyl rings and $\mathrm{N}-\mathrm{H} \cdots \mathrm{N}$ intermolecular interactions which are displayed. Additional weak $\pi-\pi C g 1$ (pyrimidine) $\cdots C g 2$ (phenyl) intermolecular interactions are also present forming a two-dimensional network along (111). H atoms not involved in hydrogen bonding have been omitted for clarity 
interplanar distance of $3.464 \AA$, a centroid-to-centroid $(C g 1 \cdots C g 2 ; C g 1$ is the centroid of the $\mathrm{N} 1 A / \mathrm{C} 2 A / \mathrm{N} 3 A / \mathrm{C} 4 A-$ C6 $A$ ring and $C g 2$ is the centroid of the $\mathrm{C} 1 B-\mathrm{C} 6 B$ ring) distance of 3.5283 (8) $\AA$ and a slip angle of $9.65^{\circ}$, are also found to help stabilize the crystal structure (Fig. 34).

It is very interesting to observe two series of cocrystals involving 4-amino-5-chloro-2,6-dimethylpyrimidine and carboxylic acids. In one series of cocrystals, the carboxyl hydroxy group $(-\mathrm{OH})$ is hydrogen bonded to atom $\mathrm{N} 1(\mathrm{O}-\mathrm{H} \cdots \mathrm{N} 1)$ of the corresponding pyrimidine ring (single point supramolecular synthon). In the other series, the carboxyl group interacts with atom $\mathrm{N} 3$ and the adjacent 4-amino group of the corresponding pyrimidine via $\mathrm{O}-\mathrm{H} \cdots \mathrm{N}$ and $\mathrm{N}-\mathrm{H} \cdots \mathrm{O}$ hydrogen bonds to generate a robust $R_{2}^{2}(8)$ supramolecular heterosynthon. This is similarly reminiscent of two types of protonation, at $\mathrm{N} 1$ or $\mathrm{N} 3$ of 4-amino-5-chloro-2,6-dimethylpyrimidine, reported recently (Rajam et al., 2017). Further studies may reveal whether each of the cocrystals of one series will have a polymorphic form corresponding to the other series of cocrystals, similar to the cation tautomerism reported for this type of pyrimidine interaction (Rajam et al., 2017).

\section{Acknowledgements}

PTM thanks UGC, New Delhi, for a UGC-Emeritus Fellowship. The authors acknowledge funding from Youngstown State University and the assistance of Dr Matthias Zeller of Youngstown State University (now at Purdue University) in the collection of the diffraction data (for crystals $\mathbf{1}, \mathbf{2}$ and $\mathbf{5}$ ). RJB wishes to acknowledge the assistance of the University of Canterbury in allowing him to use their diffractometer during a visit in May 2015 (for crystals 3, 4 and 6-10).

\section{Funding information}

Funding for this research was provided by: National Science Foundation (grant No. DMR 1337296, funds to purchase the diffractometer at Youngstown State University; grant No. CHE 0087210); Ohio Board of Regents (grant No. CAP-491).

\section{References}

Aakeröy, C. B., Schultheiss, N. C., Rajbanshi, A., Desper, J. \& Moore, C. (2009). Cryst. Growth Des. 9, 432-441.

Agilent (2014). CrysAlis PRO. Agilent Technologies Ltd, Yarnton, Oxfordshire, England.

Baldrighi, M., Cavallo, G., Chierotti, M. R., Gobetto, R., Metrangolo, P., Pilati, T., Resnati, G. \& Terraneo, G. (2013). Mol. Pharm. 10, 1760-1772.

Baskar Raj, S., Muthiah, P. T., Rychlewska, U. \& Warzajtis, B. (2003). CrystEngComm, 5, 48-53.
Bruker (2013). APEX2 and SAINT. Bruker AXS Inc., Madison, Wisconsin, USA.

Bruker (2014). APEX2 and SAINT. Bruker AXS Inc., Madison, Wisconsin, USA.

Cavallo, G., Metrangolo, P., Milani, R., Pilati, T., Priimagi, A., Resnati, G. \& Terraneo, G. (2016). Chem. Rev. 116, 2478-2601.

Clark, R. C. \& Reid, J. S. (1995). Acta Cryst. A51, 887-897.

Ebenezer, S. \& Muthiah, P. T. (2012). Cryst. Growth Des. 12, 37663785.

Ebenezer, S., Muthiah, P. T. \& Butcher, R. J. (2011). Cryst. Growth Des. 11, 3579-3592.

García-Raso, A., Albertí, F. M., Fiol, J. J., Tasada, A., Barceló-Oliver, M., Molins, E., Estarellas, C., Frontera, A., Quiñonero, D. \& Deyà, P. M. (2009). Cryst. Growth Des. 9, 2363-2376.

Griffini, G., Brambilla, L., Levi, M., Castiglioni, C., Del Zoppo, M. \& Turri, S. (2014). RSC Adv. 4, 9893-9897.

Hübschle, C. B., Sheldrick, G. M. \& Dittrich, B. (2011). J. Appl. Cryst. 44, 1281-1284.

Jennifer, S. J. \& Muthiah, P. T. (2014). Chem. Cent. J. 8, article number 20.

Karthikeyan, A., Swinton Darious, R., Thomas Muthiah, P. \& Perdih, F. (2015). Acta Cryst. C71, 985-990.

Lu, Y., Shi, T., Wang, Y., Yang, H., Yan, X., Luo, X., Jiang, H. \& Zhu, W. (2009). J. Med. Chem. 52, 2854-2862.

Macrae, C. F., Bruno, I. J., Chisholm, J. A., Edgington, P. R., McCabe, P., Pidcock, E., Rodriguez-Monge, L., Taylor, R., van de Streek, J. \& Wood, P. A. (2008). J. Appl. Cryst. 41, 466-470.

Metrangolo, P. \& Resnati, G. (2012). Cryst. Growth Des. 12, 58355838 .

Mukherjee, A. \& Desiraju, G. R. (2014). Cryst. Growth Des. 14, 13751385.

Nandi, G., Titi, H. M. \& Goldberg, I. (2014). Cryst. Growth Des. 14, 3557-3566.

Oxford Diffraction (2011). CrysAlis PRO. Oxford Diffraction Ltd, Yarnton, Oxfordshire, England.

Parsons, S., Flack, H. D. \& Wagner, T. (2013). Acta Cryst. B69, 249259.

Rajam, A., Muthiah, P. T., Butcher, R. J. \& Jasinski, J. P. (2015). Acta Cryst. E71, o479-o480.

Rajam, A., Muthiah, P. T., Butcher, R. J., Jasinski, J. P. \& Glidewell, C. (2017). Acta Cryst. C73, 862-868.

Selvam, T. P., James, C. R., Dniandev, V. \& Valzita, S. K. (2012). Res. Pharm. 2, 1-9.

Sharma, V., Chitranshi, C. \& Agarwal, A. K. (2014). Int. J. Med. Chem. article number 202784.

Sheldrick, G. M. (2008). Acta Cryst. A64, 112-122.

Sheldrick, G. M. (2015). Acta Cryst. C71, 3-8.

Spek, A. L. (2009). Acta Cryst. D65, 148-155.

Stanley, N., Sethuraman, V., Muthiah, P. T., Luger, P. \& Weber, M. (2002). Cryst. Growth Des. 2, 631-635.

Swinton Darious, R., Thomas Muthiah, P. \& Perdih, F. (2017). Acta Cryst. C73, 743-748.

Tamilselvi, D. \& Muthiah, P. T. (2011). Acta Cryst. C67, o192o194.

Tothadi, S., Joseph, S. \& Desiraju, G. R. (2013). Cryst. Growth Des. 13, 3242-3254.

Zhu, W., Zheng, R., Fu, X., Fu, H., Shi, Q., Zhen, Y., Dong, H. \& Hu, W. (2015). Angew. Chem. Int. Ed. 54, 6785-6789. 


\section{supporting information}

Acta Cryst. (2018). C74, 1007-1019 [https://doi.org/10.1107/S2053229618009154]

Design of two series of 1:1 cocrystals involving 4-amino-5-chloro-2,6-dimethylpyrimidine and carboxylic acids

Ammaiyappan Rajam, Packianathan Thomas Muthiah, Raymond John Butcher, Jerry P. Jasinski and Jan Wikaira

Computing details

Data collection: APEX2 (Bruker, 2014) for COCRYSTAL-1, COCRYSTAL-2, COCRYSTAL-5; CrysAlis PRO (Agilent, 2014) for COCRYSTAL-3, COCRYSTAL-6, COCRYSTAL-7, COCRYSTAL-8, COCRYSTAL-9, COCRYSTAL-10; CrysAlis PRO (Oxford Diffraction, 2011) for COCRYSTAL-4. Cell refinement: SAINT (Bruker, 2014) for COCRYSTAL-1, COCRYSTAL-2; CrysAlis PRO (Agilent, 2014) for COCRYSTAL-3, COCRYSTAL-6, COCRYSTAL-7, COCRYSTAL-8, COCRYSTAL-9, COCRYSTAL-10; CrysAlis PRO (Oxford Diffraction, 2011) for COCRYSTAL-4; SAINT (Bruker, 2013) for COCRYSTAL-5. Data reduction: SAINT (Bruker, 2014) for COCRYSTAL-1, COCRYSTAL-2; CrysAlis PRO (Agilent, 2014) for COCRYSTAL-3, COCRYSTAL-6, COCRYSTAL-7, COCRYSTAL-8, COCRYSTAL-9, COCRYSTAL-10; CrysAlis PRO (Oxford Diffraction, 2011) for COCRYSTAL-4; SAINT (Bruker, 2013) for COCRYSTAL-5. Program(s) used to solve structure: SHELXS97 (Sheldrick, 2008) for COCRYSTAL-1, COCRYSTAL-2, COCRYSTAL-5; SHELXT (Sheldrick, 2015a) for COCRYSTAL-3, COCRYSTAL-4, COCRYSTAL-6, COCRYSTAL-7, COCRYSTAL-8, COCRYSTAL-9, COCRYSTAL-10. Program(s) used to refine structure: SHELXL2018 (Sheldrick, 2015b) for COCRYSTAL-1, COCRYSTAL-2; SHELXL2014 (Sheldrick, 2015b) for COCRYSTAL-3, COCRYSTAL-4, COCRYSTAL-6, COCRYSTAL-7, COCRYSTAL-8, COCRYSTAL-9, COCRYSTAL-10; SHELXL2014 (Sheldrick, 2015b) and shelXle (Hübschle et al., 2011) for COCRYSTAL-5. Molecular graphics: SHELXTL (Sheldrick, 2008) for COCRYSTAL-1, COCRYSTAL-2, COCRYSTAL-3, COCRYSTAL-4, COCRYSTAL-7, COCRYSTAL-8, COCRYSTAL-9, COCRYSTAL-10; SHELXT (Sheldrick, 2008) for COCRYSTAL-6. Software used to prepare material for publication: SHELXTL (Sheldrick, 2008) for COCRYSTAL-1, COCRYSTAL-2, COCRYSTAL-3, COCRYSTAL-4, COCRYSTAL-7, COCRYSTAL-8, COCRYSTAL-9, COCRYSTAL-10; SHELXT (Sheldrick, 2008) for COCRYSTAL-6.

4-Amino-5-chloro-2,6-dimethylpyrimidine-3-bromothiophene-2-carboxylic acid (1/1) (COCRYSTAL-1)

Crystal data

$\mathrm{C}_{5} \mathrm{H}_{3} \mathrm{BrO}_{2} \mathrm{~S} \cdot \mathrm{C}_{6} \mathrm{H}_{8} \mathrm{ClN}_{3}$

$Z=2$

$M_{r}=364.65$

Triclinic, $P \overline{1}$

$a=7.5237$ (4) $\AA$

$b=7.5739(4) \AA$

$c=12.5089(7) \AA$

$\alpha=79.629(2)^{\circ}$

$\beta=79.596(2)^{\circ}$

$\gamma=75.895(2)^{\circ}$

$V=673.00(6) \AA^{3}$

$F(000)=364$

$D_{\mathrm{x}}=1.799 \mathrm{Mg} \mathrm{m}^{-3}$

Mo $K \alpha$ radiation, $\lambda=0.71073 \AA$

Cell parameters from 9311 reflections

$\theta=2.8-33.2^{\circ}$

$\mu=3.41 \mathrm{~mm}^{-1}$

$T=100 \mathrm{~K}$

Block, colourless

$0.31 \times 0.27 \times 0.21 \mathrm{~mm}$ 


\section{Data collection}

Bruker D8 Quest CMOS diffractometer

Radiation source: I-mu-S microsource X-ray tube

$\omega$ and phi scans

Absorption correction: multi-scan (APEX2; Bruker, 2014)

$T_{\text {min }}=0.539, T_{\text {max }}=0.747$

\section{Refinement}

Refinement on $F^{2}$

Least-squares matrix: full

$R\left[F^{2}>2 \sigma\left(F^{2}\right)\right]=0.034$

$w R\left(F^{2}\right)=0.070$

$S=1.04$

5103 reflections

187 parameters

0 restraints

Primary atom site location: structure-invariant direct methods
13063 measured reflections

5103 independent reflections

4471 reflections with $I>2 \sigma(I)$

$R_{\text {int }}=0.032$

$\theta_{\max }=33.2^{\circ}, \theta_{\min }=2.8^{\circ}$

$h=-11 \rightarrow 10$

$k=-11 \rightarrow 11$

$l=-19 \rightarrow 19$

Secondary atom site location: difference Fourier map

Hydrogen site location: mixed

$\mathrm{H}$ atoms treated by a mixture of independent and constrained refinement

$w=1 /\left[\sigma^{2}\left(F_{\mathrm{o}}{ }^{2}\right)+(0.010 P)^{2}+0.8813 P\right]$

where $P=\left(F_{\mathrm{o}}{ }^{2}+2 F_{\mathrm{c}}{ }^{2}\right) / 3$

$(\Delta / \sigma)_{\max }<0.001$

$\Delta \rho_{\max }=0.67 \mathrm{e} \AA^{-3}$

$\Delta \rho_{\min }=-0.52$ e $\AA^{-3}$

Special details

Geometry. All esds (except the esd in the dihedral angle between two 1.s. planes) are estimated using the full covariance matrix. The cell esds are taken into account individually in the estimation of esds in distances, angles and torsion angles; correlations between esds in cell parameters are only used when they are defined by crystal symmetry. An approximate (isotropic) treatment of cell esds is used for estimating esds involving l.s. planes.

Fractional atomic coordinates and isotropic or equivalent isotropic displacement parameters $\left(\AA^{2}\right)$

\begin{tabular}{llllll}
\hline & $x$ & $y$ & $z$ & $U_{\text {iso }} / U_{\text {eq }}$ & Occ. $(<1)$ \\
\hline Br1 & $0.17359(2)$ & $-0.20786(2)$ & $0.99807(2)$ & $0.01468(5)$ & \\
S1 & $0.71218(6)$ & $-0.09607(6)$ & $0.83962(4)$ & $0.01414(9)$ & \\
O1B & $0.4649(2)$ & $0.1721(2)$ & $0.71276(12)$ & $0.0203(3)$ & \\
H1A & $0.396(5)$ & $0.264(5)$ & $0.673(3)$ & $0.050(10)^{*}$ & \\
O2B & $0.1948(2)$ & $0.1035(2)$ & $0.79633(13)$ & $0.0221(3)$ & \\
C1B & $0.3626(3)$ & $0.0767(2)$ & $0.78633(15)$ & $0.0134(3)$ & \\
C2B & $0.4747(2)$ & $-0.0686(2)$ & $0.85783(14)$ & $0.0113(3)$ & \\
C3B & $0.4191(2)$ & $-0.1903(2)$ & $0.94601(15)$ & $0.0108(3)$ & \\
C4B & $0.5662(2)$ & $-0.3038(2)$ & $0.99870(15)$ & $0.0131(3)$ & \\
H4AA & 0.550993 & -0.393948 & 1.061103 & $0.016^{*}$ & \\
C5B & $0.7327(3)$ & $-0.2678(2)$ & $0.94879(16)$ & $0.0156(3)$ & \\
H5AA & 0.847630 & -0.331186 & 0.972153 & $0.019^{*}$ & \\
C11 & $0.20623(6)$ & $0.53889(6)$ & $0.26918(4)$ & $0.01714(9)$ & \\
N1A & $0.2875(2)$ & $0.4395(2)$ & $0.58151(13)$ & $0.0135(3)$ & \\
C1A & $0.2123(2)$ & $0.6060(2)$ & $0.61071(15)$ & $0.0128(3)$ & \\
N3A & $0.1304(2)$ & $0.7519(2)$ & $0.54593(13)$ & $0.0127(3)$ & \\
N4A & $0.0334(2)$ & $0.8728(2)$ & $0.37794(14)$ & $0.0146(3)$ & \\
H4B1 & $-0.013(4)$ & $0.970(4)$ & $0.401(2)$ & $0.027(7)^{*}$ \\
H4B2 & $0.012(4)$ & $0.856(4)$ & $0.318(2)$ & $0.027(7)^{*}$ & \\
C4A & $0.1219(2)$ & $0.7307(2)$ & $0.44175(14)$ & $0.0111(3)$ &
\end{tabular}




\begin{tabular}{|c|c|c|c|c|c|}
\hline C5A & 0.2057 (2) & $0.5596(2)$ & $0.40456(14)$ & $0.0117(3)$ & \\
\hline C6A & $0.2853(2)$ & $0.4153(2)$ & $0.47652(15)$ & $0.0129(3)$ & \\
\hline C7A & $0.2190(3)$ & $0.6288(3)$ & $0.72665(16)$ & 0.0184 (4) & \\
\hline H7BA & 0.346202 & 0.583683 & 0.742949 & $0.028^{*}$ & \\
\hline H7BB & 0.137498 & 0.558307 & 0.777376 & $0.028 *$ & \\
\hline H7BC & 0.177617 & 0.759176 & 0.735254 & $0.028^{*}$ & \\
\hline $\mathrm{C} 8 \mathrm{~A}$ & $0.3708(3)$ & $0.2269(3)$ & $0.44675(17)$ & 0.0193 (4) & \\
\hline H8B1 & 0.413316 & 0.144726 & 0.511493 & $0.029 *$ & $0.50(3)$ \\
\hline H8B2 & 0.476348 & 0.233326 & 0.388531 & $0.029 *$ & $0.50(3)$ \\
\hline H8B3 & 0.278688 & 0.179407 & 0.420768 & $0.029 *$ & $0.50(3)$ \\
\hline H8B4 & 0.376237 & 0.229615 & 0.367619 & $0.029^{*}$ & $0.50(3)$ \\
\hline H8B5 & 0.295740 & 0.140367 & 0.486933 & $0.029 *$ & $0.50(3)$ \\
\hline H8B6 & 0.496375 & 0.187476 & 0.466240 & $0.029 *$ & $0.50(3)$ \\
\hline
\end{tabular}

Atomic displacement parameters $\left(\AA^{2}\right)$

\begin{tabular}{lllllll}
\hline & $U^{11}$ & $U^{22}$ & $U^{33}$ & $U^{12}$ & $U^{13}$ & $U^{23}$ \\
\hline $\mathrm{Br} 1$ & $0.01179(8)$ & $0.01544(8)$ & $0.01657(9)$ & $-0.00349(6)$ & $-0.00291(6)$ & $-0.00006(6)$ \\
$\mathrm{S} 1$ & $0.01211(18)$ & $0.01347(19)$ & $0.0168(2)$ & $-0.00285(14)$ & $-0.00211(15)$ & $-0.00203(15)$ \\
$\mathrm{O} 1 \mathrm{~B}$ & $0.0187(6)$ & $0.0178(6)$ & $0.0202(7)$ & $-0.0021(5)$ & $-0.0034(5)$ & $0.0064(5)$ \\
$\mathrm{O} 2 \mathrm{~B}$ & $0.0174(6)$ & $0.0246(7)$ & $0.0219(8)$ & $-0.0032(5)$ & $-0.0092(6)$ & $0.0072(6)$ \\
$\mathrm{C} 1 \mathrm{~B}$ & $0.0168(8)$ & $0.0131(7)$ & $0.0107(8)$ & $-0.0022(6)$ & $-0.0044(6)$ & $-0.0018(6)$ \\
$\mathrm{C} 2 \mathrm{~B}$ & $0.0122(7)$ & $0.0107(7)$ & $0.0115(8)$ & $-0.0014(6)$ & $-0.0042(6)$ & $-0.0018(6)$ \\
$\mathrm{C} 3 \mathrm{~B}$ & $0.0104(7)$ & $0.0098(7)$ & $0.0131(8)$ & $-0.0016(5)$ & $-0.0033(6)$ & $-0.0034(6)$ \\
$\mathrm{C} 4 \mathrm{~B}$ & $0.0161(7)$ & $0.0106(7)$ & $0.0139(8)$ & $-0.0024(6)$ & $-0.0075(6)$ & $-0.0002(6)$ \\
$\mathrm{C} 5 \mathrm{~B}$ & $0.0147(7)$ & $0.0130(8)$ & $0.0196(9)$ & $-0.0008(6)$ & $-0.0076(7)$ & $-0.0019(6)$ \\
$\mathrm{C} 11$ & $0.0222(2)$ & $0.0184(2)$ & $0.01073(19)$ & $-0.00160(16)$ & $-0.00411(16)$ & $-0.00395(15)$ \\
$\mathrm{N} 1 \mathrm{~A}$ & $0.0160(7)$ & $0.0110(6)$ & $0.0125(7)$ & $-0.0013(5)$ & $-0.0039(5)$ & $0.0004(5)$ \\
$\mathrm{C} 1 \mathrm{~A}$ & $0.0138(7)$ & $0.0125(7)$ & $0.0118(8)$ & $-0.0030(6)$ & $-0.0034(6)$ & $0.0006(6)$ \\
$\mathrm{N} 3 \mathrm{~A}$ & $0.0156(7)$ & $0.0112(6)$ & $0.0111(7)$ & $-0.0026(5)$ & $-0.0035(5)$ & $-0.0004(5)$ \\
$\mathrm{N} 4 \mathrm{~A}$ & $0.0205(7)$ & $0.0100(7)$ & $0.0123(7)$ & $0.0002(6)$ & $-0.0063(6)$ & $-0.0002(5)$ \\
$\mathrm{C} 4 \mathrm{~A}$ & $0.0112(7)$ & $0.0110(7)$ & $0.0111(7)$ & $-0.0030(5)$ & $-0.0019(6)$ & $-0.0005(6)$ \\
$\mathrm{C} 5 \mathrm{~A}$ & $0.0146(7)$ & $0.0115(7)$ & $0.0090(7)$ & $-0.0025(6)$ & $-0.0023(6)$ & $-0.0012(5)$ \\
$\mathrm{C} 6 \mathrm{~A}$ & $0.0131(7)$ & $0.0118(7)$ & $0.0130(8)$ & $-0.0029(6)$ & $-0.0012(6)$ & $-0.0001(6)$ \\
$\mathrm{C} 7 \mathrm{~A}$ & $0.0242(9)$ & $0.0207(9)$ & $0.0106(8)$ & $-0.0035(7)$ & $-0.0062(7)$ & $-0.0012(6)$ \\
$\mathrm{C} 8 \mathrm{~A}$ & $0.0225(9)$ & $0.0122(8)$ & $0.0203(9)$ & $0.0017(7)$ & $-0.0032(7)$ & $-0.0023(7)$ \\
& & & & & & \\
\hline
\end{tabular}

Geometric parameters $\left(\AA,{ }^{\circ}\right)$

\begin{tabular}{llll}
\hline $\mathrm{Br} 1-\mathrm{C} 3 \mathrm{~B}$ & $1.8729(17)$ & $\mathrm{C} 1 \mathrm{~A}-\mathrm{N} 3 \mathrm{~A}$ & $1.335(2)$ \\
$\mathrm{Br} 1-\mathrm{Br} 1^{\mathrm{i}}$ & $3.5693(4)$ & $\mathrm{C} 1 \mathrm{~A}-\mathrm{C} 7 \mathrm{~A}$ & $1.502(3)$ \\
$\mathrm{Br} 1-\mathrm{C} 11^{\mathrm{ii}}$ & $3.6014(5)$ & $\mathrm{N} 3 \mathrm{~A}-\mathrm{C} 4 \mathrm{~A}$ & $1.356(2)$ \\
$\mathrm{Br} 1-\mathrm{S} 1^{\mathrm{iii}}$ & $3.6656(5)$ & $\mathrm{N} 4 \mathrm{~A}-\mathrm{C} 4 \mathrm{~A}$ & $1.331(2)$ \\
$\mathrm{S} 1-\mathrm{C} 5 \mathrm{~B}$ & $1.7083(19)$ & $\mathrm{N} 4 \mathrm{~A}-\mathrm{H} 4 \mathrm{~B} 1$ & $0.81(3)$ \\
$\mathrm{S} 1-\mathrm{C} 2 \mathrm{~B}$ & $1.7253(17)$ & $\mathrm{N} 4 \mathrm{~A}-\mathrm{H} 4 \mathrm{~B} 2$ & $0.83(3)$ \\
$\mathrm{O} 1 \mathrm{~B}-\mathrm{C} 1 \mathrm{~B}$ & $1.318(2)$ & $\mathrm{C} 4 \mathrm{~A}-\mathrm{C} 5 \mathrm{~A}$ & $1.416(2)$ \\
$\mathrm{O} 1 \mathrm{~B}-\mathrm{H} 1 \mathrm{~A}$ & $0.89(3)$ & $\mathrm{C} 5 \mathrm{~A}-\mathrm{C} 6 \mathrm{~A}$ & $1.373(2)$ \\
$\mathrm{O} 2 \mathrm{~B}-\mathrm{C} 1 \mathrm{~B}$ & $1.216(2)$ & $\mathrm{C} 6 \mathrm{~A}-\mathrm{C} 8 \mathrm{~A}$ & $1.499(3)$
\end{tabular}




\begin{tabular}{|c|c|c|c|}
\hline $\mathrm{C} 1 \mathrm{~B}-\mathrm{C} 2 \mathrm{~B}$ & $1.479(2)$ & $\mathrm{C} 7 \mathrm{~A}-\mathrm{H} 7 \mathrm{BA}$ & 0.9800 \\
\hline $\mathrm{C} 2 \mathrm{~B}-\mathrm{C} 3 \mathrm{~B}$ & $1.377(2)$ & $\mathrm{C} 7 \mathrm{~A}-\mathrm{H} 7 \mathrm{BB}$ & 0.9800 \\
\hline $\mathrm{C} 3 \mathrm{~B}-\mathrm{C} 4 \mathrm{~B}$ & $1.412(2)$ & $\mathrm{C} 7 \mathrm{~A}-\mathrm{H} 7 \mathrm{BC}$ & 0.9800 \\
\hline $\mathrm{C} 4 \mathrm{~B}-\mathrm{C} 5 \mathrm{~B}$ & $1.362(3)$ & $\mathrm{C} 8 \mathrm{~A}-\mathrm{H} 8 \mathrm{~B} 1$ & 0.9800 \\
\hline $\mathrm{C} 4 \mathrm{~B}-\mathrm{H} 4 \mathrm{AA}$ & 0.9500 & $\mathrm{C} 8 \mathrm{~A}-\mathrm{H} 8 \mathrm{~B} 2$ & 0.9800 \\
\hline $\mathrm{C} 5 \mathrm{~B}-\mathrm{H} 5 \mathrm{AA}$ & 0.9500 & $\mathrm{C} 8 \mathrm{~A}-\mathrm{H} 8 \mathrm{~B} 3$ & 0.9800 \\
\hline $\mathrm{C} 11-\mathrm{C} 5 \mathrm{~A}$ & $1.7276(18)$ & $\mathrm{C} 8 \mathrm{~A}-\mathrm{H} 8 \mathrm{~B} 4$ & 0.9800 \\
\hline $\mathrm{N} 1 \mathrm{~A}-\mathrm{C} 1 \mathrm{~A}$ & $1.337(2)$ & $\mathrm{C} 8 \mathrm{~A}-\mathrm{H} 8 \mathrm{~B} 5$ & 0.9800 \\
\hline $\mathrm{N} 1 \mathrm{~A}-\mathrm{C} 6 \mathrm{~A}$ & $1.362(2)$ & $\mathrm{C} 8 \mathrm{~A}-\mathrm{H} 8 \mathrm{~B} 6$ & 0.9800 \\
\hline $\mathrm{C} 3 \mathrm{~B}-\mathrm{Br} 1-\mathrm{Br} 1^{\mathrm{i}}$ & $118.11(5)$ & $\mathrm{C} 4 \mathrm{~A}-\mathrm{N} 4 \mathrm{~A}-\mathrm{H} 4 \mathrm{~B} 2$ & $118.9(19)$ \\
\hline $\mathrm{C} 3 \mathrm{~B}-\mathrm{Br} 1-\mathrm{C} 11^{\mathrm{ii}}$ & $102.47(5)$ & $\mathrm{H} 4 \mathrm{~B} 1-\mathrm{N} 4 \mathrm{~A}-\mathrm{H} 4 \mathrm{~B} 2$ & $120(3)$ \\
\hline $\mathrm{Br} 1^{\mathrm{i}}-\mathrm{Br} 1-\mathrm{Cl} 1^{\mathrm{ii}}$ & $111.923(11)$ & $\mathrm{N} 4 \mathrm{~A}-\mathrm{C} 4 \mathrm{~A}-\mathrm{N} 3 \mathrm{~A}$ & $118.32(16)$ \\
\hline $\mathrm{C} 3 \mathrm{~B}-\mathrm{Br} 1-\mathrm{S} 1^{\mathrm{iii}}$ & $73.14(5)$ & $\mathrm{N} 4 \mathrm{~A}-\mathrm{C} 4 \mathrm{~A}-\mathrm{C} 5 \mathrm{~A}$ & $122.24(16)$ \\
\hline $\mathrm{Br} 1^{\mathrm{i}}-\mathrm{Br} 1-\mathrm{S} 1^{\mathrm{iii}}$ & $69.809(10)$ & $\mathrm{N} 3 \mathrm{~A}-\mathrm{C} 4 \mathrm{~A}-\mathrm{C} 5 \mathrm{~A}$ & $119.44(15)$ \\
\hline $\mathrm{C} 11^{\mathrm{ii}}-\mathrm{Br} 1-\mathrm{S} 1^{\mathrm{iii}}$ & $73.552(12)$ & $\mathrm{C} 6 \mathrm{~A}-\mathrm{C} 5 \mathrm{~A}-\mathrm{C} 4 \mathrm{~A}$ & $119.30(16)$ \\
\hline $\mathrm{C} 5 \mathrm{~B}-\mathrm{S} 1-\mathrm{C} 2 \mathrm{~B}$ & $91.90(9)$ & $\mathrm{C} 6 \mathrm{~A}-\mathrm{C} 5 \mathrm{~A}-\mathrm{Cl1}$ & $121.77(14)$ \\
\hline $\mathrm{C} 1 \mathrm{~B}-\mathrm{O} 1 \mathrm{~B}-\mathrm{H} 1 \mathrm{~A}$ & $112(2)$ & $\mathrm{C} 4 \mathrm{~A}-\mathrm{C} 5 \mathrm{~A}-\mathrm{C} 11$ & $118.93(13)$ \\
\hline $\mathrm{O} 2 \mathrm{~B}-\mathrm{C} 1 \mathrm{~B}-\mathrm{O} 1 \mathrm{~B}$ & $124.83(17)$ & $\mathrm{N} 1 \mathrm{~A}-\mathrm{C} 6 \mathrm{~A}-\mathrm{C} 5 \mathrm{~A}$ & $119.82(16)$ \\
\hline $\mathrm{O} 2 \mathrm{~B}-\mathrm{C} 1 \mathrm{~B}-\mathrm{C} 2 \mathrm{~B}$ & $122.74(17)$ & $\mathrm{N} 1 \mathrm{~A}-\mathrm{C} 6 \mathrm{~A}-\mathrm{C} 8 \mathrm{~A}$ & $116.65(16)$ \\
\hline $\mathrm{O} 1 \mathrm{~B}-\mathrm{C} 1 \mathrm{~B}-\mathrm{C} 2 \mathrm{~B}$ & $112.43(15)$ & $\mathrm{C} 5 \mathrm{~A}-\mathrm{C} 6 \mathrm{~A}-\mathrm{C} 8 \mathrm{~A}$ & $123.52(17)$ \\
\hline $\mathrm{C} 3 \mathrm{~B}-\mathrm{C} 2 \mathrm{~B}-\mathrm{C} 1 \mathrm{~B}$ & $129.82(16)$ & $\mathrm{C} 1 \mathrm{~A}-\mathrm{C} 7 \mathrm{~A}-\mathrm{H} 7 \mathrm{BA}$ & 109.5 \\
\hline $\mathrm{C} 3 \mathrm{~B}-\mathrm{C} 2 \mathrm{~B}-\mathrm{S} 1$ & $110.12(12)$ & $\mathrm{C} 1 \mathrm{~A}-\mathrm{C} 7 \mathrm{~A}-\mathrm{H} 7 \mathrm{BB}$ & 109.5 \\
\hline $\mathrm{C} 1 \mathrm{~B}-\mathrm{C} 2 \mathrm{~B}-\mathrm{S} 1$ & $119.99(13)$ & $\mathrm{H} 7 \mathrm{BA}-\mathrm{C} 7 \mathrm{~A}-\mathrm{H} 7 \mathrm{BB}$ & 109.5 \\
\hline $\mathrm{C} 2 \mathrm{~B}-\mathrm{C} 3 \mathrm{~B}-\mathrm{C} 4 \mathrm{~B}$ & $113.85(15)$ & $\mathrm{C} 1 \mathrm{~A}-\mathrm{C} 7 \mathrm{~A}-\mathrm{H} 7 \mathrm{BC}$ & 109.5 \\
\hline $\mathrm{C} 2 \mathrm{~B}-\mathrm{C} 3 \mathrm{~B}-\mathrm{Br} 1$ & $125.30(13)$ & $\mathrm{H} 7 \mathrm{BA}-\mathrm{C} 7 \mathrm{~A}-\mathrm{H} 7 \mathrm{BC}$ & 109.5 \\
\hline $\mathrm{C} 4 \mathrm{~B}-\mathrm{C} 3 \mathrm{~B}-\mathrm{Br} 1$ & $120.84(13)$ & $\mathrm{H} 7 \mathrm{BB}-\mathrm{C} 7 \mathrm{~A}-\mathrm{H} 7 \mathrm{BC}$ & 109.5 \\
\hline $\mathrm{C} 5 \mathrm{~B}-\mathrm{C} 4 \mathrm{~B}-\mathrm{C} 3 \mathrm{~B}$ & $111.48(16)$ & $\mathrm{C} 6 \mathrm{~A}-\mathrm{C} 8 \mathrm{~A}-\mathrm{H} 8 \mathrm{~B} 1$ & 109.5 \\
\hline $\mathrm{C} 5 \mathrm{~B}-\mathrm{C} 4 \mathrm{~B}-\mathrm{H} 4 \mathrm{AA}$ & 124.3 & $\mathrm{C} 6 \mathrm{~A}-\mathrm{C} 8 \mathrm{~A}-\mathrm{H} 8 \mathrm{~B} 2$ & 109.5 \\
\hline $\mathrm{C} 3 \mathrm{~B}-\mathrm{C} 4 \mathrm{~B}-\mathrm{H} 4 \mathrm{AA}$ & 124.3 & $\mathrm{H} 8 \mathrm{~B} 1-\mathrm{C} 8 \mathrm{~A}-\mathrm{H} 8 \mathrm{~B} 2$ & 109.5 \\
\hline $\mathrm{C} 4 \mathrm{~B}-\mathrm{C} 5 \mathrm{~B}-\mathrm{S} 1$ & $112.65(13)$ & $\mathrm{C} 6 \mathrm{~A}-\mathrm{C} 8 \mathrm{~A}-\mathrm{H} 8 \mathrm{~B} 3$ & 109.5 \\
\hline $\mathrm{C} 4 \mathrm{~B}-\mathrm{C} 5 \mathrm{~B}-\mathrm{H} 5 \mathrm{AA}$ & 123.7 & $\mathrm{H} 8 \mathrm{~B} 1-\mathrm{C} 8 \mathrm{~A}-\mathrm{H} 8 \mathrm{~B} 3$ & 109.5 \\
\hline $\mathrm{S} 1-\mathrm{C} 5 \mathrm{~B}-\mathrm{H} 5 \mathrm{AA}$ & 123.7 & $\mathrm{H} 8 \mathrm{~B} 2-\mathrm{C} 8 \mathrm{~A}-\mathrm{H} 8 \mathrm{~B} 3$ & 109.5 \\
\hline $\mathrm{C} 1 \mathrm{~A}-\mathrm{N} 1 \mathrm{~A}-\mathrm{C} 6 \mathrm{~A}$ & $118.14(15)$ & $\mathrm{C} 6 \mathrm{~A}-\mathrm{C} 8 \mathrm{~A}-\mathrm{H} 8 \mathrm{~B} 4$ & 109.5 \\
\hline $\mathrm{N} 3 \mathrm{~A}-\mathrm{C} 1 \mathrm{~A}-\mathrm{N} 1 \mathrm{~A}$ & $125.43(17)$ & $\mathrm{C} 6 \mathrm{~A}-\mathrm{C} 8 \mathrm{~A}-\mathrm{H} 8 \mathrm{~B} 5$ & 109.5 \\
\hline $\mathrm{N} 3 \mathrm{~A}-\mathrm{C} 1 \mathrm{~A}-\mathrm{C} 7 \mathrm{~A}$ & $117.73(16)$ & $\mathrm{H} 8 \mathrm{~B} 4-\mathrm{C} 8 \mathrm{~A}-\mathrm{H} 8 \mathrm{~B} 5$ & 109.5 \\
\hline $\mathrm{N} 1 \mathrm{~A}-\mathrm{C} 1 \mathrm{~A}-\mathrm{C} 7 \mathrm{~A}$ & $116.83(15)$ & $\mathrm{C} 6 \mathrm{~A}-\mathrm{C} 8 \mathrm{~A}-\mathrm{H} 8 \mathrm{~B} 6$ & 109.5 \\
\hline $\mathrm{C} 1 \mathrm{~A}-\mathrm{N} 3 \mathrm{~A}-\mathrm{C} 4 \mathrm{~A}$ & $117.78(15)$ & $\mathrm{H} 8 \mathrm{~B} 4-\mathrm{C} 8 \mathrm{~A}-\mathrm{H} 8 \mathrm{~B} 6$ & 109.5 \\
\hline $\mathrm{C} 4 \mathrm{~A}-\mathrm{N} 4 \mathrm{~A}-\mathrm{H} 4 \mathrm{~B} 1$ & $121(2)$ & $\mathrm{H} 8 \mathrm{~B} 5-\mathrm{C} 8 \mathrm{~A}-\mathrm{H} 8 \mathrm{~B} 6$ & 109.5 \\
\hline $\mathrm{O} 2 \mathrm{~B}-\mathrm{C} 1 \mathrm{~B}-\mathrm{C} 2 \mathrm{~B}-\mathrm{C} 3 \mathrm{~B}$ & $-2.5(3)$ & $\mathrm{C} 3 \mathrm{~B}-\mathrm{C} 4 \mathrm{~B}-\mathrm{C} 5 \mathrm{~B}-\mathrm{S} 1$ & $0.7(2)$ \\
\hline $\mathrm{O} 1 \mathrm{~B}-\mathrm{C} 1 \mathrm{~B}-\mathrm{C} 2 \mathrm{~B}-\mathrm{C} 3 \mathrm{~B}$ & $177.09(18)$ & $\mathrm{C} 2 \mathrm{~B}-\mathrm{S} 1-\mathrm{C} 5 \mathrm{~B}-\mathrm{C} 4 \mathrm{~B}$ & $-0.41(15)$ \\
\hline $\mathrm{O} 2 \mathrm{~B}-\mathrm{C} 1 \mathrm{~B}-\mathrm{C} 2 \mathrm{~B}-\mathrm{S} 1$ & $-179.15(15)$ & $\mathrm{C} 6 \mathrm{~A}-\mathrm{N} 1 \mathrm{~A}-\mathrm{C} 1 \mathrm{~A}-\mathrm{N} 3 \mathrm{~A}$ & $1.6(3)$ \\
\hline $\mathrm{O} 1 \mathrm{~B}-\mathrm{C} 1 \mathrm{~B}-\mathrm{C} 2 \mathrm{~B}-\mathrm{S} 1$ & $0.4(2)$ & $\mathrm{C} 6 \mathrm{~A}-\mathrm{N} 1 \mathrm{~A}-\mathrm{C} 1 \mathrm{~A}-\mathrm{C} 7 \mathrm{~A}$ & $-179.29(16)$ \\
\hline $\mathrm{C} 5 \mathrm{~B}-\mathrm{S} 1-\mathrm{C} 2 \mathrm{~B}-\mathrm{C} 3 \mathrm{~B}$ & $0.04(14)$ & $\mathrm{N} 1 \mathrm{~A}-\mathrm{C} 1 \mathrm{~A}-\mathrm{N} 3 \mathrm{~A}-\mathrm{C} 4 \mathrm{~A}$ & $0.1(3)$ \\
\hline $\mathrm{C} 5 \mathrm{~B}-\mathrm{S} 1-\mathrm{C} 2 \mathrm{~B}-\mathrm{C} 1 \mathrm{~B}$ & $177.34(15)$ & $\mathrm{C} 7 \mathrm{~A}-\mathrm{C} 1 \mathrm{~A}-\mathrm{N} 3 \mathrm{~A}-\mathrm{C} 4 \mathrm{~A}$ & $-179.07(16)$ \\
\hline $\mathrm{C} 1 \mathrm{~B}-\mathrm{C} 2 \mathrm{~B}-\mathrm{C} 3 \mathrm{~B}-\mathrm{C} 4 \mathrm{~B}$ & $-176.64(17)$ & $\mathrm{C} 1 \mathrm{~A}-\mathrm{N} 3 \mathrm{~A}-\mathrm{C} 4 \mathrm{~A}-\mathrm{N} 4 \mathrm{~A}$ & $177.07(16)$ \\
\hline $\mathrm{S} 1-\mathrm{C} 2 \mathrm{~B}-\mathrm{C} 3 \mathrm{~B}-\mathrm{C} 4 \mathrm{~B}$ & $0.32(19)$ & $\mathrm{C} 1 \mathrm{~A}-\mathrm{N} 3 \mathrm{~A}-\mathrm{C} 4 \mathrm{~A}-\mathrm{C} 5 \mathrm{~A}$ & $-2.6(2)$ \\
\hline
\end{tabular}




$\begin{array}{llll}\mathrm{C} 1 \mathrm{~B}-\mathrm{C} 2 \mathrm{~B}-\mathrm{C} 3 \mathrm{~B}-\mathrm{Br} 1 & 2.0(3) & \mathrm{N} 4 \mathrm{~A}-\mathrm{C} 4 \mathrm{~A}-\mathrm{C} 5 \mathrm{~A}-\mathrm{C} 6 \mathrm{~A} & -176.19(17) \\ \mathrm{S} 1-\mathrm{C} 2 \mathrm{~B}-\mathrm{C} 3 \mathrm{~B}-\mathrm{Br} 1 & 178.93(9) & \mathrm{N} 3 \mathrm{~A}-\mathrm{C} 4 \mathrm{~A}-\mathrm{C} 5 \mathrm{~A}-\mathrm{C} 6 \mathrm{~A} & 3.4(3) \\ \mathrm{Br} 1 \mathrm{i}-\mathrm{Br} 1-\mathrm{C} 3 \mathrm{~B}-\mathrm{C} 2 \mathrm{~B} & -39.37(17) & \mathrm{N} 4 \mathrm{~A}-\mathrm{C} 4 \mathrm{~A}-\mathrm{C} 5 \mathrm{~A}-\mathrm{C} 11 & 4.3(2) \\ \mathrm{C} 11^{\mathrm{ii}}-\mathrm{Br} 1-\mathrm{C} 3 \mathrm{~B}-\mathrm{C} 2 \mathrm{~B} & -162.85(14) & \mathrm{N} 3 \mathrm{~A}-\mathrm{C} 4 \mathrm{~A}-\mathrm{C} 5 \mathrm{~A}-\mathrm{C} 11 & -176.06(13) \\ \mathrm{S} 1^{\mathrm{ii}}-\mathrm{Br} 1-\mathrm{C} 3 \mathrm{~B}-\mathrm{C} 2 \mathrm{~B} & -94.57(15) & \mathrm{C} 1 \mathrm{~A}-\mathrm{N} 1 \mathrm{~A}-\mathrm{C} 6 \mathrm{~A}-\mathrm{C} 5 \mathrm{~A} & -0.6(3) \\ \mathrm{Br} 1 \mathrm{i}-\mathrm{Br} 1-\mathrm{C} 3 \mathrm{~B}-\mathrm{C} 4 \mathrm{~B} & 139.14(12) & \mathrm{C} 1 \mathrm{~A}-\mathrm{N} 1 \mathrm{~A}-\mathrm{C} 6 \mathrm{~A}-\mathrm{C} 8 \mathrm{~A} & -179.97(16) \\ \mathrm{C} 11^{\mathrm{ii}}-\mathrm{Br} 1-\mathrm{C} 3 \mathrm{~B}-\mathrm{C} 4 \mathrm{~B} & 15.66(15) & \mathrm{C} 4 \mathrm{~A}-\mathrm{C} 5 \mathrm{~A}-\mathrm{C} 6 \mathrm{~A}-\mathrm{N} 1 \mathrm{~A} & -1.8(3) \\ \mathrm{S} 1{ }^{\mathrm{iii}}-\mathrm{Br} 1-\mathrm{C} 3 \mathrm{~B}-\mathrm{C} 4 \mathrm{~B} & 83.94(14) & \mathrm{C} 11-\mathrm{C} 5 \mathrm{~A}-\mathrm{C} 6 \mathrm{~A}-\mathrm{N} 1 \mathrm{~A} & 177.67(13) \\ \mathrm{C} 2 \mathrm{~B}-\mathrm{C} 3 \mathrm{~B}-\mathrm{C} 4 \mathrm{~B}-\mathrm{C} 5 \mathrm{~B} & -0.6(2) & \mathrm{C} 4 \mathrm{~A}-\mathrm{C} 5 \mathrm{~A}-\mathrm{C} 6 \mathrm{~A}-\mathrm{C} 8 \mathrm{~A} & 177.52(17) \\ \mathrm{Br} 1-\mathrm{C} 3 \mathrm{~B}-\mathrm{C} 4 \mathrm{~B}-\mathrm{C} 5 \mathrm{~B} & -179.30(13) & \mathrm{C} 11-\mathrm{C} 5 \mathrm{~A}-\mathrm{C} 6 \mathrm{~A}-\mathrm{C} 8 \mathrm{~A} & -3.0(3)\end{array}$

Symmetry codes: (i) $-x,-y,-z+2$; (ii) $x, y-1, z+1$; (iii) $-x+1,-y,-z+2$.

Hydrogen-bond geometry $\left(\AA,{ }^{\circ}\right)$

\begin{tabular}{lllll}
\hline$D-\mathrm{H} \cdots A$ & $D-\mathrm{H}$ & $\mathrm{H} \cdots A$ & $D \cdots A$ & $D-\mathrm{H} \cdots A$ \\
\hline $\mathrm{O} 1 B-\mathrm{H} 1 A \cdots \mathrm{N} 1 A$ & $0.89(3)$ & $1.73(4)$ & $2.618(2)$ & $172(3)$ \\
$\mathrm{C} 5 B-\mathrm{H} 5 A A \cdots \mathrm{Br} 1^{\text {iv }}$ & 0.95 & 2.92 & $3.6281(18)$ & 133 \\
$\mathrm{~N} 4 A-\mathrm{H} 4 B 1 \cdots \mathrm{N} 3 A^{\mathrm{v}}$ & $0.81(3)$ & $2.25(3)$ & $3.058(2)$ & $176(3)$ \\
$\mathrm{N} 4 A-\mathrm{H} 4 B 2 \cdots \mathrm{O} 2 B^{\mathrm{vi}}$ & $0.83(3)$ & $2.23(3)$ & $2.965(2)$ & $148(3)$ \\
$\mathrm{C} 8 A-\mathrm{H} 8 B 4 \cdots \mathrm{C} 11$ & 0.98 & 2.59 & $3.119(2)$ & 114 \\
\hline
\end{tabular}

Symmetry codes: (iv) $x+1, y, z$; (v) $-x,-y+2,-z+1 ;$ (vi) $-x,-y+1,-z+1$.

4-Amino-5-chloro-2,6-dimethylpyrimidine-5-chlorothiophene-2-carboxylic acid (1/1) (COCRYSTAL-2)

Crystal data

$\mathrm{C}_{6} \mathrm{H}_{8} \mathrm{ClN}_{3} \cdot \mathrm{C}_{5} \mathrm{H}_{3} \mathrm{ClO}_{2} \mathrm{~S}$

$M_{r}=320.19$

Triclinic, $P \overline{1}$

$a=7.2182(3) \AA$

$b=7.6364(3) \AA$

$c=12.7516(6) \AA$

$\alpha=90.707(2)^{\circ}$

$\beta=102.629(2)^{\circ}$

$\gamma=105.338(2)^{\circ}$

$V=659.61(5) \AA^{3}$

\section{Data collection}

Bruker D8 Quest CMOS diffractometer

Radiation source: I-mu-S microsource X-ray tube

$\omega$ and phi scans

Absorption correction: multi-scan

(APEX2; Bruker, 2014)

$T_{\text {min }}=0.477, T_{\max }=0.746$

Refinement

Refinement on $F^{2}$

Least-squares matrix: full

$R\left[F^{2}>2 \sigma\left(F^{2}\right)\right]=0.051$

$w R\left(F^{2}\right)=0.138$
$Z=2$

$F(000)=328$

$D_{\mathrm{x}}=1.612 \mathrm{Mg} \mathrm{m}^{-3}$

Mo $K \alpha$ radiation, $\lambda=0.71073 \AA$

Cell parameters from 5911 reflections

$\theta=2.8-29.6^{\circ}$

$\mu=0.65 \mathrm{~mm}^{-1}$

$T=100 \mathrm{~K}$

Rod, colourless

$0.37 \times 0.19 \times 0.11 \mathrm{~mm}$

8796 measured reflections

3636 independent reflections

2947 reflections with $I>2 \sigma(I)$

$R_{\text {int }}=0.051$

$\theta_{\text {max }}=29.6^{\circ}, \theta_{\min }=2.8^{\circ}$

$h=-10 \rightarrow 9$

$k=-10 \rightarrow 10$

$l=-17 \rightarrow 17$

$S=1.15$

3636 reflections

187 parameters

0 restraints 
Primary atom site location: structure-invariant direct methods

Secondary atom site location: difference Fourier map

Hydrogen site location: mixed
$\mathrm{H}$ atoms treated by a mixture of independent and constrained refinement

$w=1 /\left[\sigma^{2}\left(F_{\mathrm{o}}^{2}\right)+(0.0554 P)^{2}+0.5883 P\right]$

where $P=\left(F_{\mathrm{o}}^{2}+2 F_{\mathrm{c}}^{2}\right) / 3$

$(\Delta / \sigma)_{\max }<0.001$

$\Delta \rho_{\max }=0.76 \mathrm{e} \AA^{-3}$

$\Delta \rho_{\min }=-0.56$ e $\AA^{-3}$

Special details

Geometry. All esds (except the esd in the dihedral angle between two 1.s. planes) are estimated using the full covariance matrix. The cell esds are taken into account individually in the estimation of esds in distances, angles and torsion angles; correlations between esds in cell parameters are only used when they are defined by crystal symmetry. An approximate (isotropic) treatment of cell esds is used for estimating esds involving 1.s. planes.

Fractional atomic coordinates and isotropic or equivalent isotropic displacement parameters $\left(\AA^{2}\right)$

\begin{tabular}{llllll}
\hline & $x$ & $y$ & $z$ & $U_{\text {iso }} * / U_{\text {eq }}$ & Occ. $(<1)$ \\
\hline C11B & $-0.28477(8)$ & $-0.34293(8)$ & $0.00245(5)$ & $0.01882(16)$ & \\
S1B & $0.04024(8)$ & $-0.07368(8)$ & $0.15616(5)$ & $0.01421(15)$ & \\
O1B & $0.3910(3)$ & $0.1819(2)$ & $0.29123(14)$ & $0.0189(4)$ & \\
H1A & $0.498(6)$ & $0.281(6)$ & $0.332(4)$ & $0.059(13)^{*}$ & \\
O2B & $0.6159(2)$ & $0.1262(3)$ & $0.20660(15)$ & $0.0212(4)$ & \\
C1B & $0.4469(3)$ & $0.0974(3)$ & $0.21763(19)$ & $0.0155(5)$ & \\
C2B & $0.2832(3)$ & $-0.0399(3)$ & $0.14660(18)$ & $0.0133(4)$ & \\
C3B & $0.2971(3)$ & $-0.1483(3)$ & $0.06501(19)$ & $0.0153(4)$ & \\
H3AA & 0.418792 & -0.147819 & 0.047805 & $0.018^{*}$ & \\
C4B & $0.1128(3)$ & $-0.2620(3)$ & $0.00817(19)$ & $0.0161(5)$ & \\
H4AA & 0.096144 & -0.346000 & -0.050993 & $0.019^{*}$ & \\
C5B & $-0.0382(3)$ & $-0.2356(3)$ & $0.04925(18)$ & $0.0142(4)$ & \\
C11A & $0.94838(8)$ & $0.51403(8)$ & $0.72886(4)$ & $0.01724(15)$ & \\
N1A & $0.6580(3)$ & $0.4397(3)$ & $0.42204(16)$ & $0.0134(4)$ & \\
C2A & $0.7088(3)$ & $0.6114(3)$ & $0.39334(18)$ & $0.0128(4)$ & \\
N3A & $0.8311(3)$ & $0.7539(3)$ & $0.45660(15)$ & $0.0133(4)$ & \\
N4A & $1.0414(3)$ & $0.8636(3)$ & $0.62157(16)$ & $0.0144(4)$ & \\
H4B1 & $1.076(5)$ & $0.974(5)$ & $0.602(3)$ & $0.035(9)^{*}$ & \\
H4B2 & $1.119(4)$ & $0.855(4)$ & $0.683(3)$ & $0.021(8)^{*}$ & \\
C4A & $0.9120(3)$ & $0.7244(3)$ & $0.55926(18)$ & $0.0117(4)$ & \\
C5A & $0.8567(3)$ & $0.5476(3)$ & $0.59597(17)$ & $0.0119(4)$ & \\
C6A & $0.7326(3)$ & $0.4068(3)$ & $0.52538(18)$ & $0.0135(4)$ & \\
C7A & $0.6204(4)$ & $0.6424(4)$ & $0.27936(19)$ & $0.0177(5)$ & \\
H7BA & 0.652565 & 0.773352 & 0.270051 & $0.027^{*}$ & \\
H7BB & 0.476747 & 0.591891 & 0.264024 & $0.027^{*}$ & \\
H7BC & 0.674362 & 0.582116 & 0.229662 & $0.027^{*}$ & \\
C8A & $0.6708(4)$ & $0.2145(3)$ & $0.5550(2)$ & $0.0171(5)$ & \\
H8B1 & 0.610279 & 0.133235 & 0.489367 & $0.026^{*}$ & \\
H8B2 & 0.574907 & 0.204034 & 0.600045 & $0.026^{*}$ & \\
H8B3 & 0.786587 & 0.180211 & 0.595054 & $0.026^{*}$ & \\
H8B4 & 0.718728 & 0.209791 & 0.632726 & $0.026^{*}$ & \\
H8B5 & 0.726639 & 0.137893 & 0.515809 & $0.026^{*}$ & \\
& & & & \\
& & & \\
\end{tabular}


Atomic displacement parameters $\left(\AA^{2}\right)$

\begin{tabular}{lllllll}
\hline & $U^{11}$ & $U^{22}$ & $U^{33}$ & $U^{12}$ & $U^{13}$ & $U^{23}$ \\
\hline C11B & $0.0139(3)$ & $0.0185(3)$ & $0.0179(3)$ & $-0.0015(2)$ & $-0.0017(2)$ & $-0.0007(2)$ \\
S1B & $0.0142(3)$ & $0.0142(3)$ & $0.0121(3)$ & $0.0010(2)$ & $0.0022(2)$ & $-0.0021(2)$ \\
O1B & $0.0167(8)$ & $0.0180(9)$ & $0.0170(9)$ & $-0.0026(7)$ & $0.0025(7)$ & $-0.0078(7)$ \\
O2B & $0.0129(8)$ & $0.0256(10)$ & $0.0209(9)$ & $0.0020(7)$ & $-0.0009(7)$ & $-0.0037(7)$ \\
C1B & $0.0169(11)$ & $0.0118(11)$ & $0.0139(11)$ & $0.0007(9)$ & $-0.0007(8)$ & $0.0001(9)$ \\
C2B & $0.0114(10)$ & $0.0147(11)$ & $0.0116(10)$ & $0.0018(8)$ & $-0.0002(8)$ & $0.0022(8)$ \\
C3B & $0.0141(10)$ & $0.0150(11)$ & $0.0158(11)$ & $0.0036(9)$ & $0.0022(8)$ & $0.0009(9)$ \\
C4B & $0.0195(11)$ & $0.0152(11)$ & $0.0099(10)$ & $0.0023(9)$ & $-0.0013(8)$ & $-0.0041(9)$ \\
C5B & $0.0143(10)$ & $0.0127(11)$ & $0.0118(10)$ & $0.0007(8)$ & $-0.0015(8)$ & $0.0001(8)$ \\
C11A & $0.0200(3)$ & $0.0183(3)$ & $0.0099(3)$ & $0.0019(2)$ & $0.0002(2)$ & $0.0015(2)$ \\
N1A & $0.0117(9)$ & $0.0128(9)$ & $0.0131(9)$ & $-0.0008(7)$ & $0.0028(7)$ & $-0.0024(7)$ \\
C2A & $0.0107(10)$ & $0.0156(11)$ & $0.0106(10)$ & $0.0022(8)$ & $0.0014(8)$ & $-0.0028(8)$ \\
N3A & $0.0122(9)$ & $0.0143(9)$ & $0.0120(9)$ & $0.0018(7)$ & $0.0022(7)$ & $0.0001(7)$ \\
N4A & $0.0167(9)$ & $0.0121(10)$ & $0.0101(9)$ & $-0.0005(8)$ & $-0.0002(7)$ & $-0.0025(8)$ \\
C4A & $0.0105(10)$ & $0.0125(10)$ & $0.0120(10)$ & $0.0036(8)$ & $0.0018(8)$ & $-0.0009(8)$ \\
C5A & $0.0127(10)$ & $0.0145(11)$ & $0.0071(9)$ & $0.0025(8)$ & $0.0010(7)$ & $-0.0001(8)$ \\
C6A & $0.0142(10)$ & $0.0142(11)$ & $0.0122(10)$ & $0.0026(9)$ & $0.0046(8)$ & $-0.0008(8)$ \\
C7A & $0.0178(11)$ & $0.0220(12)$ & $0.0118(11)$ & $0.0045(10)$ & $0.0013(8)$ & $0.0029(9)$ \\
C8A & $0.0193(11)$ & $0.0103(11)$ & $0.0171(11)$ & $-0.0026(9)$ & $0.0031(9)$ & $0.0006(9)$ \\
& & & & & &
\end{tabular}

Geometric parameters $\left(\AA,{ }^{\circ}\right)$

\begin{tabular}{llll}
\hline C11B-C5B & $1.717(2)$ & $\mathrm{N} 3 \mathrm{~A}-\mathrm{C} 4 \mathrm{~A}$ & $1.360(3)$ \\
$\mathrm{S} 1 \mathrm{~B}-\mathrm{C} 5 \mathrm{~B}$ & $1.720(2)$ & $\mathrm{N} 4 \mathrm{~A}-\mathrm{C} 4 \mathrm{~A}$ & $1.328(3)$ \\
$\mathrm{S} 1 \mathrm{~B}-\mathrm{C} 2 \mathrm{~B}$ & $1.735(2)$ & $\mathrm{N} 4 \mathrm{~A}-\mathrm{H} 4 \mathrm{~B} 1$ & $0.87(4)$ \\
$\mathrm{O} 1 \mathrm{~B}-\mathrm{C} 1 \mathrm{~B}$ & $1.322(3)$ & $\mathrm{N} 4 \mathrm{~A}-\mathrm{H} 4 \mathrm{~B} 2$ & $0.87(3)$ \\
$\mathrm{O} 1 \mathrm{~B}-\mathrm{H} 1 \mathrm{~A}$ & $0.97(5)$ & $\mathrm{C} 4 \mathrm{~A}-\mathrm{C} 5 \mathrm{~A}$ & $1.419(3)$ \\
$\mathrm{O} 2 \mathrm{~B}-\mathrm{C} 1 \mathrm{~B}$ & $1.221(3)$ & $\mathrm{C} 5 \mathrm{~A}-\mathrm{C} 6 \mathrm{~A}$ & $1.372(3)$ \\
$\mathrm{C} 1 \mathrm{~B}-\mathrm{C} 2 \mathrm{~B}$ & $1.473(3)$ & $\mathrm{C} 6 \mathrm{~A}-\mathrm{C} 8 \mathrm{~A}$ & $1.497(3)$ \\
$\mathrm{C} 2 \mathrm{~B}-\mathrm{C} 3 \mathrm{~B}$ & $1.359(3)$ & $\mathrm{C} 7 \mathrm{~A}-\mathrm{H} 7 \mathrm{BA}$ & 0.9800 \\
$\mathrm{C} 3 \mathrm{~B}-\mathrm{C} 4 \mathrm{~B}$ & $1.414(3)$ & $\mathrm{C} 7 \mathrm{~A}-\mathrm{H} 7 \mathrm{BB}$ & 0.9800 \\
$\mathrm{C} 3 \mathrm{~B}-\mathrm{H} 3 \mathrm{AA}$ & 0.9500 & $\mathrm{C} 7 \mathrm{~A}-\mathrm{H} 7 \mathrm{BC}$ & 0.9800 \\
$\mathrm{C} 4 \mathrm{~B}-\mathrm{C} 5 \mathrm{~B}$ & $1.366(3)$ & $\mathrm{C} 8 \mathrm{~A}-\mathrm{H} 8 \mathrm{~B} 1$ & 0.9800 \\
$\mathrm{C} 4 \mathrm{~B}-\mathrm{H} 4 \mathrm{AA}$ & 0.9500 & $\mathrm{C} 8 \mathrm{~A}-\mathrm{H} 8 \mathrm{~B} 2$ & 0.9800 \\
$\mathrm{C} 11 \mathrm{~A}-\mathrm{C} 5 \mathrm{~A}$ & $1.728(2)$ & $\mathrm{C} 8 \mathrm{~A}-\mathrm{H} 8 \mathrm{~B} 3$ & 0.9800 \\
$\mathrm{~N} 1 \mathrm{~A}-\mathrm{C} 2 \mathrm{~A}$ & $1.343(3)$ & $\mathrm{C} 8 \mathrm{~A}-\mathrm{H} 8 \mathrm{~B} 4$ & 0.9800 \\
$\mathrm{~N} 1 \mathrm{~A}-\mathrm{C} 6 \mathrm{~A}$ & $1.364(3)$ & $\mathrm{C} 8 \mathrm{~A}-\mathrm{H} 8 \mathrm{~B} 5$ & 0.9800 \\
$\mathrm{C} 2 \mathrm{~A}-\mathrm{N} 3 \mathrm{~A}$ & $1.332(3)$ & $\mathrm{C} 8 \mathrm{~A}-\mathrm{H} 8 \mathrm{~B} 6$ & 0.9800 \\
$\mathrm{C} 2 \mathrm{~A}-\mathrm{C} 7 \mathrm{~A}$ & $1.503(3)$ & & $122.4(2)$ \\
& & & $119.4(2)$ \\
$\mathrm{C} 5 \mathrm{~B}-\mathrm{S} 1 \mathrm{~B}-\mathrm{C} 2 \mathrm{~B}$ & $90.45(11)$ & $\mathrm{N} 4 \mathrm{~A}-\mathrm{C} 4 \mathrm{~A}-\mathrm{C} 5 \mathrm{~A}$ & $119.5(2)$ \\
$\mathrm{C} 1 \mathrm{~B}-\mathrm{O} 1 \mathrm{~B}-\mathrm{H} 1 \mathrm{~A}$ & $113(3)$ & $\mathrm{N} 3 \mathrm{~A}-\mathrm{C} 4 \mathrm{~A}-\mathrm{C} 5 \mathrm{~A}$ & \\
$\mathrm{O} 2 \mathrm{~B}-\mathrm{C} 1 \mathrm{~B}-\mathrm{O} 1 \mathrm{~B}$ & $125.2(2)$ & $\mathrm{C} 6 \mathrm{~A}-\mathrm{C} 5 \mathrm{~A}-\mathrm{C} 4 \mathrm{~A}$ & \\
\end{tabular}




$\begin{array}{ll}\text { O2B-C1B-C2B } & 121.2(2) \\ \text { O1B-C1B-C2B } & 113.6(2) \\ \text { C3B-C2B-C1B } & 126.5(2) \\ \text { C3B-C2B-S1B } & 111.65(18) \\ \text { C1B-C2B-S1B } & 121.84(18) \\ \text { C2B-C3B-C4B } & 113.5(2) \\ \text { C2B-C3B-H3AA } & 123.3 \\ \text { C4B-C3B-H3AA } & 123.3 \\ \text { C5B-C4B-C3B } & 111.3(2) \\ \text { C5B-C4B-H4AA } & 124.3 \\ \text { C3B-C4B-H4AA } & 124.3 \\ \text { C4B-C5B-C11B } & 126.31(19) \\ \text { C4B-C5B-S1B } & 113.09(18) \\ \text { C11B-C5B-S1B } & 120.59(14) \\ \text { C2A-N1A-C6A } & 118.1(2) \\ \text { N3A-C2A-N1A } & 125.6(2) \\ \text { N3A-C2A-C7A } & 118.0(2) \\ \text { N1A-C2A-C7A } & 116.4(2) \\ \text { C2A-N3A-C4A } & 117.65(19) \\ \text { C4A-N4A-H4B1 } & 124(2) \\ \text { C4A-N4A-H4B2 } & 125(2) \\ \text { H4B1-N4A-H4B2 } & 110(3) \\ \text { N4A-C4A-N3A } & 118.2(2) \\ \text { O2B-C1B-C2B-C3B } & 0.8(4) \\ \text { O1B-C1B-C2B-C3B } & -179.3(2) \\ \text { O2B-C1B-C2B-S1B } & -176.30(18) \\ \text { O1B-C1B-C2B-S1B } & 3.6(3) \\ \text { C5B-S1B-C2B-C3B } & 0.25(18) \\ \text { C5B-S1B-C2B-C1B } & 177.78(19) \\ \text { C1B-C2B-C3B-C4B } & -177.6(2) \\ \text { S1B-C2B-C3B-C4B } & -0.2(3) \\ \text { C2B-C3B-C4B-C5B } & 0.0(3) \\ \text { C3B-C4B-C5B-C11B } & 178.81(17) \\ \text { C3B-C4B-C5B-S1B } & 0.1(3) \\ \text { C2B-S1B-C5B-C4B } & -0.22(19) \\ \text { C2B-S1B-C5B-C11B } & -178.97(15) \\ \text { C6A-N1A-C2A-N3A } & 1.8(3) \\ \text { C6A-N1A-C2A-C7A } & -178.94(19) \\ & \end{array}$

$\begin{array}{ll}\text { C6A-C5A-C11A } & 121.36(18) \\ \text { C4A-C5A-C11A } & 119.13(17) \\ \text { N1A-C6A-C5A } & 119.7(2) \\ \text { N1A-C6A-C8A } & 116.7(2) \\ \text { C5A-C6A-C8A } & 123.7(2) \\ \text { C2A-C7A-H7BA } & 109.5 \\ \text { C2A-C7A-H7BB } & 109.5 \\ \text { H7BA-C7A-H7BB } & 109.5 \\ \text { C2A-C7A-H7BC } & 109.5 \\ \text { H7BA-C7A-H7BC } & 109.5 \\ \text { H7BB-C7A-H7BC } & 109.5 \\ \text { C6A-C8A-H8B1 } & 109.5 \\ \text { C6A-C8A-H8B2 } & 109.5 \\ \text { H8B1-C8A-H8B2 } & 109.5 \\ \text { C6A-C8A-H8B3 } & 109.5 \\ \text { H8B1-C8A-H8B3 } & 109.5 \\ \text { H8B2-C8A-H8B3 } & 109.5 \\ \text { C6A-C8A-H8B4 } & 109.5 \\ \text { C6A-C8A-H8B5 } & 109.5 \\ \text { H8B4-C8A-H8B5 } & 109.5 \\ \text { C6A-C8A-H8B6 } & 109.5 \\ \text { H8B4-C8A-H8B6 } & 109.5 \\ \text { H8B5-C8A-H8B6 } & 109.5 \\ \text { N1A-C2A-N3A-C4A } & -0.1(3) \\ \text { C7A-C2A-N3A-C4A } & -179.31(19) \\ \text { C2A-N3A-C4A-N4A } & 176.8(2) \\ \text { C2A-N3A-C4A-C5A } & -2.9(3) \\ \text { N4A-C4A-C5A-C6A } & -175.5(2) \\ \text { N3A-C4A-C5A-C6A } & 4.3(3) \\ \text { N4A-C4A-C5A-C11A } & 4.2(3) \\ \text { N3A-C4A-C5A-C11A } & -176.07(16) \\ \text { C2A-N1A-C6A-C5A } & -0.4(3) \\ \text { C2A-N1A-C6A-C8A } & 179.17(19) \\ \text { C4A-C5A-C6A-N1A } & -2.5(3) \\ \text { C11A-C5A-C6A-N1A } & 177.80(16) \\ \text { C4A-C5A-C6A-C8A } & 177.9(2) \\ \text { C11A-C5A-C6A-C8A } & -1.7(3) \\ & \end{array}$

Hydrogen-bond geometry $\left(A,{ }^{\circ}\right)$

\begin{tabular}{lllll}
\hline$D-\mathrm{H} \cdots A$ & $D-\mathrm{H}$ & $\mathrm{H} \cdots A$ & $D \cdots A$ & $D-\mathrm{H} \cdots A$ \\
\hline $\mathrm{O} 1 B-\mathrm{H} 1 A \cdots \mathrm{N} 1 A$ & $0.97(5)$ & $1.66(5)$ & $2.623(3)$ & $169(4)$ \\
$\mathrm{C} 4 B-\mathrm{H} 4 A A \cdots \mathrm{C} 11 A^{\mathrm{i}}$ & 0.95 & 2.86 & $3.734(2)$ & 154 \\
$\mathrm{~N} 4 A-\mathrm{H} 4 B 1 \cdots \mathrm{N} 3 A^{\mathrm{ii}}$ & $0.87(4)$ & $2.20(4)$ & $3.070(3)$ & $176(3)$ \\
$\mathrm{N} 4 A-\mathrm{H} 4 B 2 \cdots \mathrm{O} 2 B^{\mathrm{iii}}$ & $0.87(3)$ & $2.08(3)$ & $2.907(3)$ & $157(3)$ \\
$\mathrm{C} 7 A-\mathrm{H} 7 B B \cdots \mathrm{C} 11 A^{\text {iv }}$ & 0.98 & 2.98 & $3.938(2)$ & 166
\end{tabular}




$\begin{array}{llllr}\mathrm{C} 7 A-\mathrm{H} 7 B C \cdots \mathrm{Cl} 1 A^{\mathrm{iii}} & 0.98 & 2.94 & 3.638(2) & 129 \\ \mathrm{C} 8 A-\mathrm{H} 8 B 4 \cdots \mathrm{C} 11 A & 0.98 & 2.58 & 3.110(3) & 114 \\ \mathrm{C} 8 A-\mathrm{H} 8 B 5 \cdots \mathrm{N} 4 A^{\mathrm{iii}} & 0.98 & 2.68 & 3.523(3) & 145\end{array}$

Symmetry codes: (i) $x-1, y-1, z-1$; (ii) $-x+2,-y+2,-z+1$; (iii) $-x+2,-y+1,-z+1$; (iv) $-x+1,-y+1,-z+1$.

4-Amino-5-chloro-2,6-dimethylpyrimidine-2,4-dichlorobenzoic acid (1/1) (COCRYSTAL-3)

Crystal data

$\mathrm{C}_{6} \mathrm{H}_{8} \mathrm{ClN}_{3} \cdot \mathrm{C}_{7} \mathrm{H}_{4} \mathrm{Cl}_{2} \mathrm{O}_{2}$

$M_{r}=348.61$

Triclinic, $P \overline{1}$

$a=7.3015(4) \AA$

$b=7.5060(6) \AA$

$c=14.9665(8) \AA$

$\alpha=92.539(5)^{\circ}$

$\beta=98.522(5)^{\circ}$

$\gamma=113.657(7)^{\circ}$

$V=738.12(9) \AA^{3}$

Data collection

Agilent SuperNova Dual Source diffractometer with an Atlas detector

Radiation source: sealed X-ray tube

Detector resolution: 10.6501 pixels $\mathrm{mm}^{-1}$

$\omega$ scans

Absorption correction: multi-scan

(CrysAlis PRO; Agilent, 2014)

$T_{\min }=0.538, T_{\max }=1.000$

Refinement

Refinement on $F^{2}$

Least-squares matrix: full

$R\left[F^{2}>2 \sigma\left(F^{2}\right)\right]=0.047$

$w R\left(F^{2}\right)=0.135$

$S=1.05$

3050 reflections

204 parameters

0 restraints
$Z=2$

$F(000)=356$

$D_{\mathrm{x}}=1.568 \mathrm{Mg} \mathrm{m}^{-3}$

$\mathrm{Cu} K \alpha$ radiation, $\lambda=1.54184 \AA$

Cell parameters from 2184 reflections

$\theta=3.0-75.8^{\circ}$

$\mu=5.70 \mathrm{~mm}^{-1}$

$T=120 \mathrm{~K}$

Plate, colorless

$0.51 \times 0.35 \times 0.08 \mathrm{~mm}$

5965 measured reflections

3050 independent reflections

2546 reflections with $I>2 \sigma(I)$

$R_{\text {int }}=0.038$

$\theta_{\max }=76.9^{\circ}, \theta_{\min }=3.0^{\circ}$

$h=-4 \rightarrow 9$

$k=-9 \rightarrow 8$

$l=-18 \rightarrow 17$

Hydrogen site location: mixed

$\mathrm{H}$ atoms treated by a mixture of independent and constrained refinement

$w=1 /\left[\sigma^{2}\left(F_{\mathrm{o}}^{2}\right)+(0.0781 P)^{2}+0.423 P\right]$

where $P=\left(F_{\mathrm{o}}^{2}+2 F_{\mathrm{c}}^{2}\right) / 3$

$(\Delta / \sigma)_{\max }<0.001$

$\Delta \rho_{\max }=0.39$ e $\AA^{-3}$

$\Delta \rho_{\min }=-0.51 \mathrm{e} \AA^{-3}$

Special details

Geometry. All esds (except the esd in the dihedral angle between two 1.s. planes) are estimated using the full covariance matrix. The cell esds are taken into account individually in the estimation of esds in distances, angles and torsion angles; correlations between esds in cell parameters are only used when they are defined by crystal symmetry. An approximate (isotropic) treatment of cell esds is used for estimating esds involving l.s. planes.

Fractional atomic coordinates and isotropic or equivalent isotropic displacement parameters $\left(\AA^{2}\right)$

\begin{tabular}{lllll}
\hline & $x$ & $y$ & $z$ & $U_{\text {iso }} * / U_{\text {eq }}$ \\
\hline C11 & $0.92360(9)$ & $0.67568(9)$ & $1.08079(4)$ & $0.02879(18)$ \\
N1A & $0.5642(3)$ & $0.4615(3)$ & $0.84009(15)$ & $0.0255(5)$ \\
C2A & $0.4796(4)$ & $0.2733(4)$ & $0.85176(17)$ & $0.0249(5)$ \\
N3A & $0.5203(3)$ & $0.1957(3)$ & $0.92717(15)$ & $0.0239(4)$
\end{tabular}




$\begin{array}{lllll}\text { N4A } & 0.6923(4) & 0.2415(4) & 1.07384(16) & 0.0278(5) \\ \text { H41 } & 0.624(5) & 0.118(5) & 1.077(2) & 0.024(8)^{*} \\ \text { H42 } & 0.760(6) & 0.318(6) & 1.124(3) & 0.047(11)^{*} \\ \text { C4A } & 0.6537(4) & 0.3192(4) & 0.99820(17) & 0.0232(5) \\ \text { C5A } & 0.7488(4) & 0.5206(4) & 0.98982(18) & 0.0246(5) \\ \text { C6A } & 0.7034(4) & 0.5891(4) & 0.90944(18) & 0.0251(5) \\ \text { C7A } & 0.3234(4) & 0.1328(4) & 0.77562(18) & 0.0295(6) \\ \text { H7AA } & 0.2789 & 0.2062 & 0.7313 & 0.044^{*} \\ \text { H7AB } & 0.2064 & 0.0450 & 0.8001 & 0.044^{*} \\ \text { H7AC } & 0.3828 & 0.0556 & 0.7458 & 0.044^{*} \\ \text { C8A } & 0.8001(5) & 0.7990(4) & 0.8943(2) & 0.0349(6) \\ \text { H8AA } & 0.7555 & 0.8135 & 0.8310 & 0.052^{*} \\ \text { H8AB } & 0.9483 & 0.8446 & 0.9068 & 0.052^{*} \\ \text { H8AC } & 0.7600 & 0.8771 & 0.9351 & 0.052^{*} \\ \text { C12 } & -0.04948(10) & 0.74463(11) & 0.61458(4) & 0.03186(19) \\ \text { C13 } & 0.20778(12) & 0.87604(12) & 0.29833(4) & 0.0372(2) \\ \text { O1B } & 0.4692(4) & 0.5681(4) & 0.68251(15) & 0.0382(5) \\ \text { H1B } & 0.503(7) & 0.540(8) & 0.739(4) & 0.080(16)^{*} \\ \text { O2B } & 0.2353(4) & 0.6429(4) & 0.73334(14) & 0.0446(6) \\ \text { C1B } & 0.2906(4) & 0.6968(4) & 0.58101(17) & 0.0243(5) \\ \text { C2B } & 0.1298(4) & 0.7480(4) & 0.54990(18) & 0.0253(5) \\ \text { C3B } & 0.1030(4) & 0.8021(4) & 0.46282(18) & 0.0275(5) \\ \text { H3BA } & -0.0074 & 0.8352 & 0.4420 & 0.033^{*} \\ \text { C4B } & 0.2400(4) & 0.8069(4) & 0.40696(17) & 0.0269(5) \\ \text { C5B } & 0.4022(4) & 0.7595(4) & 0.43580(19) & 0.0297(6) \\ \text { H5BA } & 0.4956 & 0.7643 & 0.3970 & 0.036^{*} \\ \text { C6B } & 0.4252(4) & 0.7051(4) & 0.52212(19) & 0.0280(6) \\ \text { H6BA } & 0.5361 & 0.6721 & 0.5423 & 0.034^{*} \\ \text { C7B } & 0.3271(4) & 0.6335(4) & 0.67360(18) & 0.0262(5) \\ & & & & \end{array}$

Atomic displacement parameters $\left(\AA^{2}\right)$

\begin{tabular}{lllllll}
\hline & $U^{11}$ & $U^{22}$ & $U^{33}$ & $U^{12}$ & $U^{13}$ & $U^{23}$ \\
\hline C11 & $0.0287(3)$ & $0.0286(3)$ & $0.0280(3)$ & $0.0116(3)$ & $0.0029(2)$ & $0.0030(2)$ \\
N1A & $0.0263(11)$ & $0.0300(11)$ & $0.0245(10)$ & $0.0148(9)$ & $0.0056(9)$ & $0.0107(9)$ \\
C2A & $0.0243(12)$ & $0.0306(13)$ & $0.0246(12)$ & $0.0154(10)$ & $0.0056(10)$ & $0.0079(10)$ \\
N3A & $0.0246(10)$ & $0.0270(11)$ & $0.0236(10)$ & $0.0131(9)$ & $0.0054(8)$ & $0.0093(8)$ \\
N4A & $0.0312(12)$ & $0.0272(12)$ & $0.0237(11)$ & $0.0110(10)$ & $0.0021(9)$ & $0.0100(9)$ \\
C4A & $0.0220(11)$ & $0.0282(13)$ & $0.0240(12)$ & $0.0137(10)$ & $0.0064(9)$ & $0.0075(10)$ \\
C5A & $0.0250(12)$ & $0.0271(13)$ & $0.0245(12)$ & $0.0131(10)$ & $0.0053(10)$ & $0.0056(10)$ \\
C6A & $0.0239(12)$ & $0.0281(13)$ & $0.0291(13)$ & $0.0150(10)$ & $0.0077(10)$ & $0.0098(10)$ \\
C7A & $0.0320(13)$ & $0.0322(14)$ & $0.0245(12)$ & $0.0140(11)$ & $0.0029(11)$ & $0.0076(10)$ \\
C8A & $0.0362(15)$ & $0.0311(15)$ & $0.0389(16)$ & $0.0147(12)$ & $0.0059(12)$ & $0.0141(12)$ \\
C12 & $0.0287(3)$ & $0.0419(4)$ & $0.0303(3)$ & $0.0192(3)$ & $0.0063(3)$ & $0.0086(3)$ \\
C13 & $0.0436(4)$ & $0.0511(4)$ & $0.0232(3)$ & $0.0265(3)$ & $0.0023(3)$ & $0.0115(3)$ \\
O1B & $0.0455(12)$ & $0.0577(14)$ & $0.0298(11)$ & $0.0366(11)$ & $0.0114(9)$ & $0.0233(10)$ \\
O2B & $0.0569(14)$ & $0.0731(17)$ & $0.0245(10)$ & $0.0463(13)$ & $0.0097(10)$ & $0.0133(10)$ \\
C1B & $0.0267(12)$ & $0.0211(12)$ & $0.0228(12)$ & $0.0092(10)$ & $-0.0004(9)$ & $0.0021(9)$
\end{tabular}




\begin{tabular}{lllllll} 
& & & & & \\
C2B & $0.0262(12)$ & $0.0239(12)$ & $0.0258(12)$ & $0.0109(10)$ & $0.0030(10)$ & $0.0042(9)$ \\
C3B & $0.0274(12)$ & $0.0268(13)$ & $0.0269(13)$ & $0.0123(11)$ & $-0.0025(10)$ & $0.0034(10)$ \\
C4B & $0.0342(14)$ & $0.0271(13)$ & $0.0190(11)$ & $0.0133(11)$ & $0.0007(10)$ & $0.0053(9)$ \\
C5B & $0.0317(14)$ & $0.0338(14)$ & $0.0263(13)$ & $0.0163(12)$ & $0.0048(11)$ & $0.0062(11)$ \\
C6B & $0.0290(13)$ & $0.0320(14)$ & $0.0270(13)$ & $0.0176(11)$ & $0.0016(10)$ & $0.0066(10)$ \\
C7B & $0.0301(13)$ & $0.0243(12)$ & $0.0250(12)$ & $0.0127(11)$ & $0.0026(10)$ & $0.0037(10)$ \\
\hline
\end{tabular}

Geometric parameters $\left(\AA,{ }^{o}\right)$

\begin{tabular}{|c|c|c|c|}
\hline $\mathrm{Cl} 1-\mathrm{C} 5 \mathrm{~A}$ & $1.729(3)$ & $\mathrm{C} 8 \mathrm{~A}-\mathrm{H} 8 \mathrm{AC}$ & 0.9800 \\
\hline $\mathrm{N} 1 \mathrm{~A}-\mathrm{C} 2 \mathrm{~A}$ & $1.327(4)$ & $\mathrm{Cl} 2-\mathrm{C} 2 \mathrm{~B}$ & $1.733(3)$ \\
\hline $\mathrm{N} 1 \mathrm{~A}-\mathrm{C} 6 \mathrm{~A}$ & $1.359(4)$ & $\mathrm{Cl} 3-\mathrm{C} 4 \mathrm{~B}$ & $1.740(3)$ \\
\hline $\mathrm{C} 2 \mathrm{~A}-\mathrm{N} 3 \mathrm{~A}$ & $1.341(3)$ & $\mathrm{O} 1 \mathrm{~B}-\mathrm{C} 7 \mathrm{~B}$ & $1.307(3)$ \\
\hline $\mathrm{C} 2 \mathrm{~A}-\mathrm{C} 7 \mathrm{~A}$ & $1.502(4)$ & $\mathrm{O} 1 \mathrm{~B}-\mathrm{H} 1 \mathrm{~B}$ & $0.90(5)$ \\
\hline $\mathrm{N} 3 \mathrm{~A}-\mathrm{C} 4 \mathrm{~A}$ & $1.349(3)$ & $\mathrm{O} 2 \mathrm{~B}-\mathrm{C} 7 \mathrm{~B}$ & $1.210(4)$ \\
\hline $\mathrm{N} 4 \mathrm{~A}-\mathrm{C} 4 \mathrm{~A}$ & $1.340(3)$ & $\mathrm{C} 1 \mathrm{~B}-\mathrm{C} 6 \mathrm{~B}$ & $1.399(4)$ \\
\hline $\mathrm{N} 4 \mathrm{~A}-\mathrm{H} 41$ & $0.87(4)$ & $\mathrm{C} 1 \mathrm{~B}-\mathrm{C} 2 \mathrm{~B}$ & $1.400(4)$ \\
\hline $\mathrm{N} 4 \mathrm{~A}-\mathrm{H} 42$ & $0.87(4)$ & $\mathrm{C} 1 \mathrm{~B}-\mathrm{C} 7 \mathrm{~B}$ & $1.506(3)$ \\
\hline $\mathrm{C} 4 \mathrm{~A}-\mathrm{C} 5 \mathrm{~A}$ & $1.408(4)$ & $\mathrm{C} 2 \mathrm{~B}-\mathrm{C} 3 \mathrm{~B}$ & $1.392(4)$ \\
\hline $\mathrm{C} 5 \mathrm{~A}-\mathrm{C} 6 \mathrm{~A}$ & $1.377(4)$ & $\mathrm{C} 3 \mathrm{~B}-\mathrm{C} 4 \mathrm{~B}$ & $1.387(4)$ \\
\hline $\mathrm{C} 6 \mathrm{~A}-\mathrm{C} 8 \mathrm{~A}$ & $1.489(4)$ & $\mathrm{C} 3 \mathrm{~B}-\mathrm{H} 3 \mathrm{BA}$ & 0.9500 \\
\hline C7A-H7AA & 0.9800 & $\mathrm{C} 4 \mathrm{~B}-\mathrm{C} 5 \mathrm{~B}$ & $1.384(4)$ \\
\hline $\mathrm{C} 7 \mathrm{~A}-\mathrm{H} 7 \mathrm{AB}$ & 0.9800 & $\mathrm{C} 5 \mathrm{~B}-\mathrm{C} 6 \mathrm{~B}$ & $1.379(4)$ \\
\hline $\mathrm{C} 7 \mathrm{~A}-\mathrm{H} 7 \mathrm{AC}$ & 0.9800 & $\mathrm{C} 5 \mathrm{~B}-\mathrm{H} 5 \mathrm{BA}$ & 0.9500 \\
\hline $\mathrm{C} 8 \mathrm{~A}-\mathrm{H} 8 \mathrm{AA}$ & 0.9800 & C6B-H6BA & 0.9500 \\
\hline $\mathrm{C} 8 \mathrm{~A}-\mathrm{H} 8 \mathrm{AB}$ & 0.9800 & & \\
\hline $\mathrm{C} 2 \mathrm{~A}-\mathrm{N} 1 \mathrm{~A}-\mathrm{C} 6 \mathrm{~A}$ & $118.5(2)$ & $\mathrm{C} 6 \mathrm{~A}-\mathrm{C} 8 \mathrm{~A}-\mathrm{H} 8 \mathrm{AC}$ & 109.5 \\
\hline $\mathrm{N} 1 \mathrm{~A}-\mathrm{C} 2 \mathrm{~A}-\mathrm{N} 3 \mathrm{~A}$ & $125.4(2)$ & $\mathrm{H} 8 \mathrm{AA}-\mathrm{C} 8 \mathrm{~A}-\mathrm{H} 8 \mathrm{AC}$ & 109.5 \\
\hline $\mathrm{N} 1 \mathrm{~A}-\mathrm{C} 2 \mathrm{~A}-\mathrm{C} 7 \mathrm{~A}$ & $118.4(2)$ & $\mathrm{H} 8 \mathrm{AB}-\mathrm{C} 8 \mathrm{~A}-\mathrm{H} 8 \mathrm{AC}$ & 109.5 \\
\hline $\mathrm{N} 3 \mathrm{~A}-\mathrm{C} 2 \mathrm{~A}-\mathrm{C} 7 \mathrm{~A}$ & $116.2(2)$ & $\mathrm{C} 7 \mathrm{~B}-\mathrm{O} 1 \mathrm{~B}-\mathrm{H} 1 \mathrm{~B}$ & $115(3)$ \\
\hline $\mathrm{C} 2 \mathrm{~A}-\mathrm{N} 3 \mathrm{~A}-\mathrm{C} 4 \mathrm{~A}$ & $117.6(2)$ & $\mathrm{C} 6 \mathrm{~B}-\mathrm{C} 1 \mathrm{~B}-\mathrm{C} 2 \mathrm{~B}$ & $117.7(2)$ \\
\hline $\mathrm{C} 4 \mathrm{~A}-\mathrm{N} 4 \mathrm{~A}-\mathrm{H} 41$ & $119(2)$ & $\mathrm{C} 6 \mathrm{~B}-\mathrm{C} 1 \mathrm{~B}-\mathrm{C} 7 \mathrm{~B}$ & $118.3(2)$ \\
\hline $\mathrm{C} 4 \mathrm{~A}-\mathrm{N} 4 \mathrm{~A}-\mathrm{H} 42$ & $119(3)$ & $\mathrm{C} 2 \mathrm{~B}-\mathrm{C} 1 \mathrm{~B}-\mathrm{C} 7 \mathrm{~B}$ & $124.0(2)$ \\
\hline $\mathrm{H} 41-\mathrm{N} 4 \mathrm{~A}-\mathrm{H} 42$ & $119(3)$ & $\mathrm{C} 3 \mathrm{~B}-\mathrm{C} 2 \mathrm{~B}-\mathrm{C} 1 \mathrm{~B}$ & $121.0(2)$ \\
\hline $\mathrm{N} 4 \mathrm{~A}-\mathrm{C} 4 \mathrm{~A}-\mathrm{N} 3 \mathrm{~A}$ & $117.5(2)$ & $\mathrm{C} 3 \mathrm{~B}-\mathrm{C} 2 \mathrm{~B}-\mathrm{Cl} 2$ & $115.8(2)$ \\
\hline $\mathrm{N} 4 \mathrm{~A}-\mathrm{C} 4 \mathrm{~A}-\mathrm{C} 5 \mathrm{~A}$ & $122.9(2)$ & $\mathrm{C} 1 \mathrm{~B}-\mathrm{C} 2 \mathrm{~B}-\mathrm{Cl} 2$ & $123.2(2)$ \\
\hline $\mathrm{N} 3 \mathrm{~A}-\mathrm{C} 4 \mathrm{~A}-\mathrm{C} 5 \mathrm{~A}$ & $119.6(2)$ & $\mathrm{C} 4 \mathrm{~B}-\mathrm{C} 3 \mathrm{~B}-\mathrm{C} 2 \mathrm{~B}$ & $118.9(2)$ \\
\hline $\mathrm{C} 6 \mathrm{~A}-\mathrm{C} 5 \mathrm{~A}-\mathrm{C} 4 \mathrm{~A}$ & $119.6(2)$ & $\mathrm{C} 4 \mathrm{~B}-\mathrm{C} 3 \mathrm{~B}-\mathrm{H} 3 \mathrm{BA}$ & 120.5 \\
\hline $\mathrm{C} 6 \mathrm{~A}-\mathrm{C} 5 \mathrm{~A}-\mathrm{C} 11$ & $121.5(2)$ & $\mathrm{C} 2 \mathrm{~B}-\mathrm{C} 3 \mathrm{~B}-\mathrm{H} 3 \mathrm{BA}$ & 120.5 \\
\hline $\mathrm{C} 4 \mathrm{~A}-\mathrm{C} 5 \mathrm{~A}-\mathrm{Cl1}$ & $118.9(2)$ & $\mathrm{C} 5 \mathrm{~B}-\mathrm{C} 4 \mathrm{~B}-\mathrm{C} 3 \mathrm{~B}$ & $121.6(2)$ \\
\hline $\mathrm{N} 1 \mathrm{~A}-\mathrm{C} 6 \mathrm{~A}-\mathrm{C} 5 \mathrm{~A}$ & $119.3(2)$ & $\mathrm{C} 5 \mathrm{~B}-\mathrm{C} 4 \mathrm{~B}-\mathrm{Cl} 3$ & $119.4(2)$ \\
\hline $\mathrm{N} 1 \mathrm{~A}-\mathrm{C} 6 \mathrm{~A}-\mathrm{C} 8 \mathrm{~A}$ & $117.7(2)$ & $\mathrm{C} 3 \mathrm{~B}-\mathrm{C} 4 \mathrm{~B}-\mathrm{Cl} 3$ & $119.0(2)$ \\
\hline $\mathrm{C} 5 \mathrm{~A}-\mathrm{C} 6 \mathrm{~A}-\mathrm{C} 8 \mathrm{~A}$ & $123.0(3)$ & $\mathrm{C} 6 \mathrm{~B}-\mathrm{C} 5 \mathrm{~B}-\mathrm{C} 4 \mathrm{~B}$ & $118.5(3)$ \\
\hline $\mathrm{C} 2 \mathrm{~A}-\mathrm{C} 7 \mathrm{~A}-\mathrm{H} 7 \mathrm{AA}$ & 109.5 & $\mathrm{C} 6 \mathrm{~B}-\mathrm{C} 5 \mathrm{~B}-\mathrm{H} 5 \mathrm{BA}$ & 120.8 \\
\hline $\mathrm{C} 2 \mathrm{~A}-\mathrm{C} 7 \mathrm{~A}-\mathrm{H} 7 \mathrm{AB}$ & 109.5 & $\mathrm{C} 4 \mathrm{~B}-\mathrm{C} 5 \mathrm{~B}-\mathrm{H} 5 \mathrm{BA}$ & 120.8 \\
\hline $\mathrm{H} 7 \mathrm{AA}-\mathrm{C} 7 \mathrm{~A}-\mathrm{H} 7 \mathrm{AB}$ & 109.5 & $\mathrm{C} 5 \mathrm{~B}-\mathrm{C} 6 \mathrm{~B}-\mathrm{C} 1 \mathrm{~B}$ & $122.2(3)$ \\
\hline $\mathrm{C} 2 \mathrm{~A}-\mathrm{C} 7 \mathrm{~A}-\mathrm{H} 7 \mathrm{AC}$ & 109.5 & $\mathrm{C} 5 \mathrm{~B}-\mathrm{C} 6 \mathrm{~B}-\mathrm{H} 6 \mathrm{BA}$ & 118.9 \\
\hline
\end{tabular}




\begin{tabular}{|c|c|c|c|}
\hline $\mathrm{H} 7 \mathrm{AA}-\mathrm{C} 7 \mathrm{~A}-\mathrm{H} 7 \mathrm{AC}$ & 109.5 & $\mathrm{C} 1 \mathrm{~B}-\mathrm{C} 6 \mathrm{~B}-\mathrm{H} 6 \mathrm{BA}$ & 118.9 \\
\hline $\mathrm{H} 7 \mathrm{AB}-\mathrm{C} 7 \mathrm{~A}-\mathrm{H} 7 \mathrm{AC}$ & 109.5 & $\mathrm{O} 2 \mathrm{~B}-\mathrm{C} 7 \mathrm{~B}-\mathrm{O} 1 \mathrm{~B}$ & $123.7(2)$ \\
\hline $\mathrm{C} 6 \mathrm{~A}-\mathrm{C} 8 \mathrm{~A}-\mathrm{H} 8 \mathrm{AA}$ & 109.5 & $\mathrm{O} 2 \mathrm{~B}-\mathrm{C} 7 \mathrm{~B}-\mathrm{C} 1 \mathrm{~B}$ & $123.7(2)$ \\
\hline $\mathrm{C} 6 \mathrm{~A}-\mathrm{C} 8 \mathrm{~A}-\mathrm{H} 8 \mathrm{AB}$ & 109.5 & $\mathrm{O} 1 \mathrm{~B}-\mathrm{C} 7 \mathrm{~B}-\mathrm{C} 1 \mathrm{~B}$ & $112.7(2)$ \\
\hline $\mathrm{H} 8 \mathrm{AA}-\mathrm{C} 8 \mathrm{~A}-\mathrm{H} 8 \mathrm{AB}$ & 109.5 & & \\
\hline $\mathrm{C} 6 \mathrm{~A}-\mathrm{N} 1 \mathrm{~A}-\mathrm{C} 2 \mathrm{~A}-\mathrm{N} 3 \mathrm{~A}$ & $-0.2(4)$ & $\mathrm{C} 7 \mathrm{~B}-\mathrm{C} 1 \mathrm{~B}-\mathrm{C} 2 \mathrm{~B}-\mathrm{C} 3 \mathrm{~B}$ & $-178.9(2)$ \\
\hline $\mathrm{C} 6 \mathrm{~A}-\mathrm{N} 1 \mathrm{~A}-\mathrm{C} 2 \mathrm{~A}-\mathrm{C} 7 \mathrm{~A}$ & $179.1(2)$ & $\mathrm{C} 6 \mathrm{~B}-\mathrm{C} 1 \mathrm{~B}-\mathrm{C} 2 \mathrm{~B}-\mathrm{Cl} 2$ & $179.8(2)$ \\
\hline $\mathrm{N} 1 \mathrm{~A}-\mathrm{C} 2 \mathrm{~A}-\mathrm{N} 3 \mathrm{~A}-\mathrm{C} 4 \mathrm{~A}$ & $2.0(4)$ & $\mathrm{C} 7 \mathrm{~B}-\mathrm{C} 1 \mathrm{~B}-\mathrm{C} 2 \mathrm{~B}-\mathrm{C} 12$ & $-0.1(4)$ \\
\hline $\mathrm{C} 7 \mathrm{~A}-\mathrm{C} 2 \mathrm{~A}-\mathrm{N} 3 \mathrm{~A}-\mathrm{C} 4 \mathrm{~A}$ & $-177.3(2)$ & $\mathrm{C} 1 \mathrm{~B}-\mathrm{C} 2 \mathrm{~B}-\mathrm{C} 3 \mathrm{~B}-\mathrm{C} 4 \mathrm{~B}$ & $-0.7(4)$ \\
\hline $\mathrm{C} 2 \mathrm{~A}-\mathrm{N} 3 \mathrm{~A}-\mathrm{C} 4 \mathrm{~A}-\mathrm{N} 4 \mathrm{~A}$ & $179.3(2)$ & $\mathrm{Cl} 2-\mathrm{C} 2 \mathrm{~B}-\mathrm{C} 3 \mathrm{~B}-\mathrm{C} 4 \mathrm{~B}$ & $-179.6(2)$ \\
\hline $\mathrm{C} 2 \mathrm{~A}-\mathrm{N} 3 \mathrm{~A}-\mathrm{C} 4 \mathrm{~A}-\mathrm{C} 5 \mathrm{~A}$ & $-2.0(4)$ & $\mathrm{C} 2 \mathrm{~B}-\mathrm{C} 3 \mathrm{~B}-\mathrm{C} 4 \mathrm{~B}-\mathrm{C} 5 \mathrm{~B}$ & $-0.1(4)$ \\
\hline $\mathrm{N} 4 \mathrm{~A}-\mathrm{C} 4 \mathrm{~A}-\mathrm{C} 5 \mathrm{~A}-\mathrm{C} 6 \mathrm{~A}$ & $179.1(2)$ & $\mathrm{C} 2 \mathrm{~B}-\mathrm{C} 3 \mathrm{~B}-\mathrm{C} 4 \mathrm{~B}-\mathrm{Cl} 3$ & $-179.6(2)$ \\
\hline $\mathrm{N} 3 \mathrm{~A}-\mathrm{C} 4 \mathrm{~A}-\mathrm{C} 5 \mathrm{~A}-\mathrm{C} 6 \mathrm{~A}$ & $0.4(4)$ & $\mathrm{C} 3 \mathrm{~B}-\mathrm{C} 4 \mathrm{~B}-\mathrm{C} 5 \mathrm{~B}-\mathrm{C} 6 \mathrm{~B}$ & $0.5(4)$ \\
\hline $\mathrm{N} 4 \mathrm{~A}-\mathrm{C} 4 \mathrm{~A}-\mathrm{C} 5 \mathrm{~A}-\mathrm{Cl1}$ & $0.1(4)$ & $\mathrm{Cl} 3-\mathrm{C} 4 \mathrm{~B}-\mathrm{C} 5 \mathrm{~B}-\mathrm{C} 6 \mathrm{~B}$ & $180.0(2)$ \\
\hline $\mathrm{N} 3 \mathrm{~A}-\mathrm{C} 4 \mathrm{~A}-\mathrm{C} 5 \mathrm{~A}-\mathrm{Cl1}$ & $-178.59(19)$ & $\mathrm{C} 4 \mathrm{~B}-\mathrm{C} 5 \mathrm{~B}-\mathrm{C} 6 \mathrm{~B}-\mathrm{C} 1 \mathrm{~B}$ & $-0.1(4)$ \\
\hline $\mathrm{C} 2 \mathrm{~A}-\mathrm{N} 1 \mathrm{~A}-\mathrm{C} 6 \mathrm{~A}-\mathrm{C} 5 \mathrm{~A}$ & $-1.5(4)$ & $\mathrm{C} 2 \mathrm{~B}-\mathrm{C} 1 \mathrm{~B}-\mathrm{C} 6 \mathrm{~B}-\mathrm{C} 5 \mathrm{~B}$ & $-0.6(4)$ \\
\hline $\mathrm{C} 2 \mathrm{~A}-\mathrm{N} 1 \mathrm{~A}-\mathrm{C} 6 \mathrm{~A}-\mathrm{C} 8 \mathrm{~A}$ & $178.6(2)$ & $\mathrm{C} 7 \mathrm{~B}-\mathrm{C} 1 \mathrm{~B}-\mathrm{C} 6 \mathrm{~B}-\mathrm{C} 5 \mathrm{~B}$ & $179.3(3)$ \\
\hline $\mathrm{C} 4 \mathrm{~A}-\mathrm{C} 5 \mathrm{~A}-\mathrm{C} 6 \mathrm{~A}-\mathrm{N} 1 \mathrm{~A}$ & $1.4(4)$ & $\mathrm{C} 6 \mathrm{~B}-\mathrm{C} 1 \mathrm{~B}-\mathrm{C} 7 \mathrm{~B}-\mathrm{O} 2 \mathrm{~B}$ & $171.1(3)$ \\
\hline $\mathrm{C} 11-\mathrm{C} 5 \mathrm{~A}-\mathrm{C} 6 \mathrm{~A}-\mathrm{N} 1 \mathrm{~A}$ & $-179.66(19)$ & $\mathrm{C} 2 \mathrm{~B}-\mathrm{C} 1 \mathrm{~B}-\mathrm{C} 7 \mathrm{~B}-\mathrm{O} 2 \mathrm{~B}$ & $-8.9(4)$ \\
\hline $\mathrm{C} 4 \mathrm{~A}-\mathrm{C} 5 \mathrm{~A}-\mathrm{C} 6 \mathrm{~A}-\mathrm{C} 8 \mathrm{~A}$ & $-178.8(2)$ & $\mathrm{C} 6 \mathrm{~B}-\mathrm{C} 1 \mathrm{~B}-\mathrm{C} 7 \mathrm{~B}-\mathrm{O} 1 \mathrm{~B}$ & $-8.7(4)$ \\
\hline $\mathrm{C} 11-\mathrm{C} 5 \mathrm{~A}-\mathrm{C} 6 \mathrm{~A}-\mathrm{C} 8 \mathrm{~A}$ & $0.2(4)$ & $\mathrm{C} 2 \mathrm{~B}-\mathrm{C} 1 \mathrm{~B}-\mathrm{C} 7 \mathrm{~B}-\mathrm{O} 1 \mathrm{~B}$ & $171.3(3)$ \\
\hline $\mathrm{C} 6 \mathrm{~B}-\mathrm{C} 1 \mathrm{~B}-\mathrm{C} 2 \mathrm{~B}-\mathrm{C} 3 \mathrm{~B}$ & $1.1(4)$ & & \\
\hline
\end{tabular}

Hydrogen-bond geometry $\left(\AA,{ }^{\circ}\right)$

\begin{tabular}{lllll}
\hline$D-\mathrm{H} \cdots A$ & $D-\mathrm{H}$ & $\mathrm{H} \cdots A$ & $D \cdots A$ & $D-\mathrm{H} \cdots A$ \\
\hline $\mathrm{N} 4 A-\mathrm{H} 41 \cdots \mathrm{N} 3 A^{\mathrm{i}}$ & $0.87(4)$ & $2.15(4)$ & $3.014(3)$ & $172(3)$ \\
$\mathrm{N} 4 A-\mathrm{H} 42 \cdots \mathrm{O} 2 B^{\mathrm{ii}}$ & $0.87(4)$ & $2.14(4)$ & $2.884(3)$ & $143(4)$ \\
$\mathrm{O} 1 B-\mathrm{H} 1 B \cdots \mathrm{N} 1 A$ & $0.90(5)$ & $1.71(5)$ & $2.610(3)$ & $174(5)$ \\
\hline
\end{tabular}

Symmetry codes: (i) $-x+1,-y,-z+2$; (ii) $-x+1,-y+1,-z+2$.

4-Amino-5-chloro-2,6-dimethylpyrimidine-2-aminobenzoic acid (1/1) (COCRYSTAL-4)

Crystal data

$\mathrm{C}_{6} \mathrm{H}_{8} \mathrm{ClN}_{3} \cdot \mathrm{C}_{7} \mathrm{H}_{7} \mathrm{NO}_{2}$

$M_{r}=294.74$

Monoclinic, $P 2{ }_{1} / n$

$a=8.0965(2) \AA$

$b=7.2427(3) \AA$

$c=24.0738(9) \AA$

$\beta=90.831(3)^{\circ}$

$V=1411.55(9) \AA^{3}$

$Z=4$

$F(000)=616$

$D_{\mathrm{x}}=1.387 \mathrm{Mg} \mathrm{m}^{-3}$

$\mathrm{Cu} K \alpha$ radiation, $\lambda=1.54184 \AA$
Cell parameters from 3296 reflections

$\theta=3.7-76.5^{\circ}$

$\mu=2.47 \mathrm{~mm}^{-1}$

$T=120 \mathrm{~K}$

The symmetry employed for this shelxl refinement is uniquely defined by the following loop, which should always be used as a source of symmetry information in preference to the above space-group names. They are only intended as comments., pale yellow-orange $0.46 \times 0.22 \times 0.06 \mathrm{~mm}$ 


\section{Data collection}

Agilent SuperNova Dual Source diffractometer with an Atlas detector

Radiation source: sealed X-ray tube Detector resolution: 10.6501 pixels $\mathrm{mm}^{-1}$ $\omega$ scans

Absorption correction: analytical [CrysAlis PRO (Oxford Diffraction, 2011), based on expressions derived by Clark \& Reid (1995)]

\section{Refinement}

Refinement on $F^{2}$

Least-squares matrix: full

$R\left[F^{2}>2 \sigma\left(F^{2}\right)\right]=0.039$

$w R\left(F^{2}\right)=0.110$

$S=1.05$

2927 reflections

203 parameters

0 restraints
$T_{\min }=0.547, T_{\max }=0.877$

6899 measured reflections

2927 independent reflections

2481 reflections with $I>2 \sigma(I)$

$R_{\text {int }}=0.028$

$\theta_{\text {max }}=76.7^{\circ}, \theta_{\min }=3.7^{\circ}$

$h=-10 \rightarrow 6$

$k=-8 \rightarrow 9$

$l=-30 \rightarrow 28$

Hydrogen site location: mixed

$\mathrm{H}$ atoms treated by a mixture of independent and constrained refinement

$w=1 /\left[\sigma^{2}\left(F_{0}^{2}\right)+(0.0615 P)^{2}+0.465 P\right]$

where $P=\left(F_{\mathrm{o}}{ }^{2}+2 F_{\mathrm{c}}{ }^{2}\right) / 3$

$(\Delta / \sigma)_{\max }=0.001$

$\Delta \rho_{\max }=0.23 \mathrm{e} \AA^{-3}$

$\Delta \rho_{\min }=-0.46$ e $\AA^{-3}$

Special details

Geometry. All esds (except the esd in the dihedral angle between two 1.s. planes) are estimated using the full covariance matrix. The cell esds are taken into account individually in the estimation of esds in distances, angles and torsion angles; correlations between esds in cell parameters are only used when they are defined by crystal symmetry. An approximate (isotropic) treatment of cell esds is used for estimating esds involving 1.s. planes.

Fractional atomic coordinates and isotropic or equivalent isotropic displacement parameters $\left(\AA^{2}\right)$

\begin{tabular}{lllll}
\hline & $x$ & $y$ & $z$ & $U_{\text {iso }} / U_{\text {eq }}$ \\
\hline C11 & $0.11199(5)$ & $0.49692(6)$ & $0.38012(2)$ & $0.02408(14)$ \\
N1A & $0.27995(17)$ & $0.51697(19)$ & $0.53553(6)$ & $0.0192(3)$ \\
C2A & $0.22132(19)$ & $0.6818(2)$ & $0.55060(7)$ & $0.0198(3)$ \\
N3A & $0.12834(16)$ & $0.79300(19)$ & $0.51880(6)$ & $0.0189(3)$ \\
N4A & $-0.00933(18)$ & $0.8419(2)$ & $0.43543(6)$ & $0.0213(3)$ \\
H4A1 & $-0.048(3)$ & $0.946(4)$ & $0.4501(10)$ & $0.034(6)^{*}$ \\
H4A2 & $-0.050(3)$ & $0.800(4)$ & $0.4043(11)$ & $0.042(7)^{*}$ \\
C4A & $0.08865(19)$ & $0.7343(2)$ & $0.46684(7)$ & $0.0179(3)$ \\
C5A & $0.15295(19)$ & $0.5643(2)$ & $0.44778(7)$ & $0.0187(3)$ \\
C6A & $0.24751(19)$ & $0.4579(2)$ & $0.48289(7)$ & $0.0194(3)$ \\
C7A & $0.2642(2)$ & $0.7482(3)$ & $0.60803(7)$ & $0.0259(4)$ \\
H7AA & 0.1778 & 0.8320 & 0.6209 & $0.039^{*}$ \\
H7AB & 0.3701 & 0.8137 & 0.6075 & $0.039^{*}$ \\
H7AC & 0.2728 & 0.6423 & 0.6333 & $0.039^{*}$ \\
C8A & $0.3162(2)$ & $0.2746(3)$ & $0.46605(7)$ & $0.0246(4)$ \\
H8AA & 0.3960 & 0.2322 & 0.4943 & $0.037^{*}$ \\
H8AB & 0.3715 & 0.2869 & 0.4303 & $0.037^{*}$ \\
H8AC & 0.2262 & 0.1847 & 0.4625 & $0.037^{*}$ \\
O1B & $0.46209(15)$ & $0.30141(19)$ & $0.59902(5)$ & $0.0268(3)$ \\
H1B & $0.379(4)$ & $0.383(5)$ & $0.5759(14)$ & $0.083(11)^{*}$ \\
O2B & $0.25284(14)$ & $0.25583(18)$ & $0.65694(5)$ & $0.0242(3)$
\end{tabular}




$\begin{array}{lllll}\text { N1B } & 0.2989(2) & 0.0084(2) & 0.74083(7) & 0.0299(4) \\ \text { H1B1 } & 0.225(3) & 0.086(4) & 0.7235(10) & 0.038(6)^{*} \\ \text { H1B2 } & 0.275(3) & -0.052(4) & 0.7695(11) & 0.042(7)^{*} \\ \text { C1B } & 0.50618(19) & 0.0925(2) & 0.67226(7) & 0.0188(3) \\ \text { C2B } & 0.4524(2) & -0.0091(2) & 0.71897(7) & 0.0200(3) \\ \text { C3B } & 0.5643(2) & -0.1364(2) & 0.74338(7) & 0.0244(4) \\ \text { H3BA } & 0.5304 & -0.2075 & 0.7744 & 0.029^{*} \\ \text { C4B } & 0.7206(2) & -0.1595(3) & 0.72327(8) & 0.0265(4) \\ \text { H4BA } & 0.7931 & -0.2459 & 0.7406 & 0.032^{*} \\ \text { C5B } & 0.7748(2) & -0.0575(3) & 0.67756(8) & 0.0250(4) \\ \text { H5BA } & 0.8837 & -0.0726 & 0.6641 & 0.030^{*} \\ \text { C6B } & 0.6672(2) & 0.0649(2) & 0.65261(7) & 0.0208(3) \\ \text { H6BA } & 0.7026 & 0.1329 & 0.6212 & 0.025^{*} \\ \text { C7B } & 0.3958(2) & 0.2228(2) & 0.64266(7) & 0.0197(3) \\ \end{array}$

Atomic displacement parameters $\left(\AA^{2}\right)$

\begin{tabular}{lllllll}
\hline & $U^{11}$ & $U^{22}$ & $U^{33}$ & $U^{12}$ & $U^{13}$ & $U^{23}$ \\
\hline C11 & $0.0307(2)$ & $0.0245(2)$ & $0.0170(2)$ & $0.00267(15)$ & $-0.00203(16)$ & $-0.00299(15)$ \\
N1A & $0.0199(6)$ & $0.0193(7)$ & $0.0182(7)$ & $0.0009(5)$ & $-0.0010(5)$ & $0.0013(5)$ \\
C2A & $0.0193(7)$ & $0.0194(8)$ & $0.0207(8)$ & $-0.0019(6)$ & $-0.0001(6)$ & $0.0002(6)$ \\
N3A & $0.0204(6)$ & $0.0183(7)$ & $0.0180(7)$ & $0.0001(5)$ & $-0.0005(5)$ & $0.0011(5)$ \\
N4A & $0.0240(7)$ & $0.0209(7)$ & $0.0189(7)$ & $0.0035(6)$ & $-0.0033(6)$ & $0.0008(6)$ \\
C4A & $0.0179(7)$ & $0.0177(8)$ & $0.0182(7)$ & $-0.0020(6)$ & $0.0013(6)$ & $0.0017(6)$ \\
C5A & $0.0210(7)$ & $0.0185(8)$ & $0.0165(7)$ & $-0.0010(6)$ & $0.0010(6)$ & $-0.0003(6)$ \\
C6A & $0.0189(7)$ & $0.0196(8)$ & $0.0197(8)$ & $-0.0011(6)$ & $0.0021(6)$ & $0.0005(6)$ \\
C7A & $0.0307(9)$ & $0.0244(9)$ & $0.0225(8)$ & $0.0027(7)$ & $-0.0060(7)$ & $-0.0031(7)$ \\
C8A & $0.0263(8)$ & $0.0227(9)$ & $0.0248(8)$ & $0.0045(7)$ & $0.0006(7)$ & $-0.0014(7)$ \\
O1B & $0.0246(6)$ & $0.0320(7)$ & $0.0239(6)$ & $0.0051(5)$ & $0.0017(5)$ & $0.0103(5)$ \\
O2B & $0.0229(6)$ & $0.0283(7)$ & $0.0213(6)$ & $0.0043(5)$ & $0.0006(5)$ & $0.0003(5)$ \\
N1B & $0.0282(8)$ & $0.0341(9)$ & $0.0275(8)$ & $0.0037(7)$ & $0.0068(7)$ & $0.0111(7)$ \\
C1B & $0.0214(7)$ & $0.0181(8)$ & $0.0170(7)$ & $-0.0003(6)$ & $-0.0025(6)$ & $-0.0017(6)$ \\
C2B & $0.0231(8)$ & $0.0195(8)$ & $0.0175(8)$ & $-0.0015(6)$ & $-0.0024(6)$ & $-0.0015(6)$ \\
C3B & $0.0324(9)$ & $0.0213(9)$ & $0.0194(8)$ & $-0.0008(7)$ & $-0.0027(7)$ & $0.0021(7)$ \\
C4B & $0.0318(9)$ & $0.0222(9)$ & $0.0252(9)$ & $0.0050(7)$ & $-0.0077(7)$ & $0.0016(7)$ \\
C5B & $0.0232(8)$ & $0.0263(9)$ & $0.0256(9)$ & $0.0053(7)$ & $-0.0010(7)$ & $-0.0006(7)$ \\
C6B & $0.0234(8)$ & $0.0202(8)$ & $0.0186(8)$ & $-0.0009(6)$ & $-0.0001(6)$ & $-0.0008(7)$ \\
C7B & $0.0218(7)$ & $0.0209(8)$ & $0.0164(7)$ & $-0.0010(6)$ & $-0.0020(6)$ & $-0.0014(6)$ \\
& & & & & &
\end{tabular}

Geometric parameters $\left(\AA,{ }^{\circ}\right)$

\begin{tabular}{llll}
\hline $\mathrm{C} 11-\mathrm{C} 5 \mathrm{~A}$ & $1.7279(16)$ & $\mathrm{O} 1 \mathrm{~B}-\mathrm{C} 7 \mathrm{~B}$ & $1.316(2)$ \\
$\mathrm{N} 1 \mathrm{~A}-\mathrm{C} 2 \mathrm{~A}$ & $1.337(2)$ & $\mathrm{O} 1 \mathrm{~B}-\mathrm{H} 1 \mathrm{~B}$ & $1.05(4)$ \\
$\mathrm{N} 1 \mathrm{~A}-\mathrm{C} 6 \mathrm{~A}$ & $1.360(2)$ & $\mathrm{O} 2 \mathrm{~B}-\mathrm{C} 7 \mathrm{~B}$ & $1.235(2)$ \\
$\mathrm{C} 2 \mathrm{~A}-\mathrm{N} 3 \mathrm{~A}$ & $1.336(2)$ & $\mathrm{N} 1 \mathrm{~B}-\mathrm{C} 2 \mathrm{~B}$ & $1.363(2)$ \\
$\mathrm{C} 2 \mathrm{~A}-\mathrm{C} 7 \mathrm{~A}$ & $1.500(2)$ & $\mathrm{N} 1 \mathrm{~B}-\mathrm{H} 1 \mathrm{~B} 1$ & $0.91(3)$ \\
$\mathrm{N} 3 \mathrm{~A}-\mathrm{C} 4 \mathrm{~A}$ & $1.355(2)$ & $\mathrm{N} 1 \mathrm{~B}-\mathrm{H} 1 \mathrm{~B} 2$ & $0.84(3)$ \\
$\mathrm{N} 4 \mathrm{~A}-\mathrm{C} 4 \mathrm{~A}$ & $1.338(2)$ & $\mathrm{C} 1 \mathrm{~B}-\mathrm{C} 6 \mathrm{~B}$ & $1.408(2)$
\end{tabular}




\begin{tabular}{|c|c|c|c|}
\hline $\mathrm{N} 4 \mathrm{~A}-\mathrm{H} 4 \mathrm{~A} 1$ & $0.89(3)$ & $\mathrm{C} 1 \mathrm{~B}-\mathrm{C} 2 \mathrm{~B}$ & $1.417(2)$ \\
\hline $\mathrm{N} 4 \mathrm{~A}-\mathrm{H} 4 \mathrm{~A} 2$ & $0.87(3)$ & $\mathrm{C} 1 \mathrm{~B}-\mathrm{C} 7 \mathrm{~B}$ & $1.476(2)$ \\
\hline $\mathrm{C} 4 \mathrm{~A}-\mathrm{C} 5 \mathrm{~A}$ & $1.416(2)$ & $\mathrm{C} 2 \mathrm{~B}-\mathrm{C} 3 \mathrm{~B}$ & $1.415(2)$ \\
\hline $\mathrm{C} 5 \mathrm{~A}-\mathrm{C} 6 \mathrm{~A}$ & $1.369(2)$ & $\mathrm{C} 3 \mathrm{~B}-\mathrm{C} 4 \mathrm{~B}$ & $1.372(3)$ \\
\hline $\mathrm{C} 6 \mathrm{~A}-\mathrm{C} 8 \mathrm{~A}$ & $1.497(2)$ & $\mathrm{C} 3 \mathrm{~B}-\mathrm{H} 3 \mathrm{BA}$ & 0.9500 \\
\hline C7A-H7AA & 0.9800 & $\mathrm{C} 4 \mathrm{~B}-\mathrm{C} 5 \mathrm{~B}$ & $1.401(3)$ \\
\hline $\mathrm{C} 7 \mathrm{~A}-\mathrm{H} 7 \mathrm{AB}$ & 0.9800 & $\mathrm{C} 4 \mathrm{~B}-\mathrm{H} 4 \mathrm{BA}$ & 0.9500 \\
\hline $\mathrm{C} 7 \mathrm{~A}-\mathrm{H} 7 \mathrm{AC}$ & 0.9800 & $\mathrm{C} 5 \mathrm{~B}-\mathrm{C} 6 \mathrm{~B}$ & $1.375(2)$ \\
\hline $\mathrm{C} 8 \mathrm{~A}-\mathrm{H} 8 \mathrm{AA}$ & 0.9800 & $\mathrm{C} 5 \mathrm{~B}-\mathrm{H} 5 \mathrm{BA}$ & 0.9500 \\
\hline $\mathrm{C} 8 \mathrm{~A}-\mathrm{H} 8 \mathrm{AB}$ & 0.9800 & $\mathrm{C} 6 \mathrm{~B}-\mathrm{H} 6 \mathrm{BA}$ & 0.9500 \\
\hline $\mathrm{C} 8 \mathrm{~A}-\mathrm{H} 8 \mathrm{AC}$ & 0.9800 & & \\
\hline $\mathrm{C} 2 \mathrm{~A}-\mathrm{N} 1 \mathrm{~A}-\mathrm{C} 6 \mathrm{~A}$ & $118.00(15)$ & $\mathrm{H} 8 \mathrm{AA}-\mathrm{C} 8 \mathrm{~A}-\mathrm{H} 8 \mathrm{AC}$ & 109.5 \\
\hline $\mathrm{N} 3 \mathrm{~A}-\mathrm{C} 2 \mathrm{~A}-\mathrm{N} 1 \mathrm{~A}$ & $125.62(16)$ & $\mathrm{H} 8 \mathrm{AB}-\mathrm{C} 8 \mathrm{~A}-\mathrm{H} 8 \mathrm{AC}$ & 109.5 \\
\hline $\mathrm{N} 3 \mathrm{~A}-\mathrm{C} 2 \mathrm{~A}-\mathrm{C} 7 \mathrm{~A}$ & $117.11(15)$ & $\mathrm{C} 7 \mathrm{~B}-\mathrm{O} 1 \mathrm{~B}-\mathrm{H} 1 \mathrm{~B}$ & $113.6(18)$ \\
\hline $\mathrm{N} 1 \mathrm{~A}-\mathrm{C} 2 \mathrm{~A}-\mathrm{C} 7 \mathrm{~A}$ & $117.28(15)$ & $\mathrm{C} 2 \mathrm{~B}-\mathrm{N} 1 \mathrm{~B}-\mathrm{H} 1 \mathrm{~B} 1$ & $118.2(15)$ \\
\hline $\mathrm{C} 2 \mathrm{~A}-\mathrm{N} 3 \mathrm{~A}-\mathrm{C} 4 \mathrm{~A}$ & $117.63(15)$ & $\mathrm{C} 2 \mathrm{~B}-\mathrm{N} 1 \mathrm{~B}-\mathrm{H} 1 \mathrm{~B} 2$ & $119.2(18)$ \\
\hline $\mathrm{C} 4 \mathrm{~A}-\mathrm{N} 4 \mathrm{~A}-\mathrm{H} 4 \mathrm{~A} 1$ & $118.4(16)$ & $\mathrm{H} 1 \mathrm{~B} 1-\mathrm{N} 1 \mathrm{~B}-\mathrm{H} 1 \mathrm{~B} 2$ & $123(2)$ \\
\hline $\mathrm{C} 4 \mathrm{~A}-\mathrm{N} 4 \mathrm{~A}-\mathrm{H} 4 \mathrm{~A} 2$ & $119.7(17)$ & $\mathrm{C} 6 \mathrm{~B}-\mathrm{C} 1 \mathrm{~B}-\mathrm{C} 2 \mathrm{~B}$ & $119.32(15)$ \\
\hline $\mathrm{H} 4 \mathrm{~A} 1-\mathrm{N} 4 \mathrm{~A}-\mathrm{H} 4 \mathrm{~A} 2$ & $121(2)$ & $\mathrm{C} 6 \mathrm{~B}-\mathrm{C} 1 \mathrm{~B}-\mathrm{C} 7 \mathrm{~B}$ & $119.05(15)$ \\
\hline $\mathrm{N} 4 \mathrm{~A}-\mathrm{C} 4 \mathrm{~A}-\mathrm{N} 3 \mathrm{~A}$ & $118.01(15)$ & $\mathrm{C} 2 \mathrm{~B}-\mathrm{C} 1 \mathrm{~B}-\mathrm{C} 7 \mathrm{~B}$ & $121.61(14)$ \\
\hline $\mathrm{N} 4 \mathrm{~A}-\mathrm{C} 4 \mathrm{~A}-\mathrm{C} 5 \mathrm{~A}$ & $122.76(15)$ & $\mathrm{N} 1 \mathrm{~B}-\mathrm{C} 2 \mathrm{~B}-\mathrm{C} 3 \mathrm{~B}$ & $118.84(16)$ \\
\hline $\mathrm{N} 3 \mathrm{~A}-\mathrm{C} 4 \mathrm{~A}-\mathrm{C} 5 \mathrm{~A}$ & $119.23(15)$ & $\mathrm{N} 1 \mathrm{~B}-\mathrm{C} 2 \mathrm{~B}-\mathrm{C} 1 \mathrm{~B}$ & $123.39(16)$ \\
\hline $\mathrm{C} 6 \mathrm{~A}-\mathrm{C} 5 \mathrm{~A}-\mathrm{C} 4 \mathrm{~A}$ & $119.62(15)$ & $\mathrm{C} 3 \mathrm{~B}-\mathrm{C} 2 \mathrm{~B}-\mathrm{C} 1 \mathrm{~B}$ & $117.77(15)$ \\
\hline $\mathrm{C} 6 \mathrm{~A}-\mathrm{C} 5 \mathrm{~A}-\mathrm{C} 11$ & $121.40(13)$ & $\mathrm{C} 4 \mathrm{~B}-\mathrm{C} 3 \mathrm{~B}-\mathrm{C} 2 \mathrm{~B}$ & $121.41(16)$ \\
\hline $\mathrm{C} 4 \mathrm{~A}-\mathrm{C} 5 \mathrm{~A}-\mathrm{Cl1}$ & $118.98(13)$ & $\mathrm{C} 4 \mathrm{~B}-\mathrm{C} 3 \mathrm{~B}-\mathrm{H} 3 \mathrm{BA}$ & 119.3 \\
\hline $\mathrm{N} 1 \mathrm{~A}-\mathrm{C} 6 \mathrm{~A}-\mathrm{C} 5 \mathrm{~A}$ & $119.79(16)$ & $\mathrm{C} 2 \mathrm{~B}-\mathrm{C} 3 \mathrm{~B}-\mathrm{H} 3 \mathrm{BA}$ & 119.3 \\
\hline $\mathrm{N} 1 \mathrm{~A}-\mathrm{C} 6 \mathrm{~A}-\mathrm{C} 8 \mathrm{~A}$ & $117.64(15)$ & $\mathrm{C} 3 \mathrm{~B}-\mathrm{C} 4 \mathrm{~B}-\mathrm{C} 5 \mathrm{~B}$ & $120.93(17)$ \\
\hline $\mathrm{C} 5 \mathrm{~A}-\mathrm{C} 6 \mathrm{~A}-\mathrm{C} 8 \mathrm{~A}$ & $122.56(15)$ & $\mathrm{C} 3 \mathrm{~B}-\mathrm{C} 4 \mathrm{~B}-\mathrm{H} 4 \mathrm{BA}$ & 119.5 \\
\hline $\mathrm{C} 2 \mathrm{~A}-\mathrm{C} 7 \mathrm{~A}-\mathrm{H} 7 \mathrm{AA}$ & 109.5 & $\mathrm{C} 5 \mathrm{~B}-\mathrm{C} 4 \mathrm{~B}-\mathrm{H} 4 \mathrm{BA}$ & 119.5 \\
\hline $\mathrm{C} 2 \mathrm{~A}-\mathrm{C} 7 \mathrm{~A}-\mathrm{H} 7 \mathrm{AB}$ & 109.5 & $\mathrm{C} 6 \mathrm{~B}-\mathrm{C} 5 \mathrm{~B}-\mathrm{C} 4 \mathrm{~B}$ & $118.65(16)$ \\
\hline H7AA-C7A-H7AB & 109.5 & $\mathrm{C} 6 \mathrm{~B}-\mathrm{C} 5 \mathrm{~B}-\mathrm{H} 5 \mathrm{BA}$ & 120.7 \\
\hline $\mathrm{C} 2 \mathrm{~A}-\mathrm{C} 7 \mathrm{~A}-\mathrm{H} 7 \mathrm{AC}$ & 109.5 & $\mathrm{C} 4 \mathrm{~B}-\mathrm{C} 5 \mathrm{~B}-\mathrm{H} 5 \mathrm{BA}$ & 120.7 \\
\hline $\mathrm{H} 7 \mathrm{AA}-\mathrm{C} 7 \mathrm{~A}-\mathrm{H} 7 \mathrm{AC}$ & 109.5 & $\mathrm{C} 5 \mathrm{~B}-\mathrm{C} 6 \mathrm{~B}-\mathrm{C} 1 \mathrm{~B}$ & $121.90(16)$ \\
\hline $\mathrm{H} 7 \mathrm{AB}-\mathrm{C} 7 \mathrm{~A}-\mathrm{H} 7 \mathrm{AC}$ & 109.5 & $\mathrm{C} 5 \mathrm{~B}-\mathrm{C} 6 \mathrm{~B}-\mathrm{H} 6 \mathrm{BA}$ & 119.0 \\
\hline C6A-C8A-H8AA & 109.5 & $\mathrm{C} 1 \mathrm{~B}-\mathrm{C} 6 \mathrm{~B}-\mathrm{H} 6 \mathrm{BA}$ & 119.0 \\
\hline $\mathrm{C} 6 \mathrm{~A}-\mathrm{C} 8 \mathrm{~A}-\mathrm{H} 8 \mathrm{AB}$ & 109.5 & $\mathrm{O} 2 \mathrm{~B}-\mathrm{C} 7 \mathrm{~B}-\mathrm{O} 1 \mathrm{~B}$ & $122.20(16)$ \\
\hline $\mathrm{H} 8 \mathrm{AA}-\mathrm{C} 8 \mathrm{~A}-\mathrm{H} 8 \mathrm{AB}$ & 109.5 & $\mathrm{O} 2 \mathrm{~B}-\mathrm{C} 7 \mathrm{~B}-\mathrm{C} 1 \mathrm{~B}$ & $123.54(15)$ \\
\hline $\mathrm{C} 6 \mathrm{~A}-\mathrm{C} 8 \mathrm{~A}-\mathrm{H} 8 \mathrm{AC}$ & 109.5 & $\mathrm{O} 1 \mathrm{~B}-\mathrm{C} 7 \mathrm{~B}-\mathrm{C} 1 \mathrm{~B}$ & $114.25(14)$ \\
\hline $\mathrm{C} 6 \mathrm{~A}-\mathrm{N} 1 \mathrm{~A}-\mathrm{C} 2 \mathrm{~A}-\mathrm{N} 3 \mathrm{~A}$ & $2.1(2)$ & $\mathrm{C} 6 \mathrm{~B}-\mathrm{C} 1 \mathrm{~B}-\mathrm{C} 2 \mathrm{~B}-\mathrm{N} 1 \mathrm{~B}$ & $-179.98(17)$ \\
\hline $\mathrm{C} 6 \mathrm{~A}-\mathrm{N} 1 \mathrm{~A}-\mathrm{C} 2 \mathrm{~A}-\mathrm{C} 7 \mathrm{~A}$ & $-177.53(14)$ & $\mathrm{C} 7 \mathrm{~B}-\mathrm{C} 1 \mathrm{~B}-\mathrm{C} 2 \mathrm{~B}-\mathrm{N} 1 \mathrm{~B}$ & $-1.7(3)$ \\
\hline $\mathrm{N} 1 \mathrm{~A}-\mathrm{C} 2 \mathrm{~A}-\mathrm{N} 3 \mathrm{~A}-\mathrm{C} 4 \mathrm{~A}$ & $0.4(2)$ & $\mathrm{C} 6 \mathrm{~B}-\mathrm{C} 1 \mathrm{~B}-\mathrm{C} 2 \mathrm{~B}-\mathrm{C} 3 \mathrm{~B}$ & $-0.8(2)$ \\
\hline $\mathrm{C} 7 \mathrm{~A}-\mathrm{C} 2 \mathrm{~A}-\mathrm{N} 3 \mathrm{~A}-\mathrm{C} 4 \mathrm{~A}$ & $-179.93(14)$ & $\mathrm{C} 7 \mathrm{~B}-\mathrm{C} 1 \mathrm{~B}-\mathrm{C} 2 \mathrm{~B}-\mathrm{C} 3 \mathrm{~B}$ & $177.48(15)$ \\
\hline $\mathrm{C} 2 \mathrm{~A}-\mathrm{N} 3 \mathrm{~A}-\mathrm{C} 4 \mathrm{~A}-\mathrm{N} 4 \mathrm{~A}$ & $177.65(14)$ & $\mathrm{N} 1 \mathrm{~B}-\mathrm{C} 2 \mathrm{~B}-\mathrm{C} 3 \mathrm{~B}-\mathrm{C} 4 \mathrm{~B}$ & $-179.78(17)$ \\
\hline $\mathrm{C} 2 \mathrm{~A}-\mathrm{N} 3 \mathrm{~A}-\mathrm{C} 4 \mathrm{~A}-\mathrm{C} 5 \mathrm{~A}$ & $-3.0(2)$ & $\mathrm{C} 1 \mathrm{~B}-\mathrm{C} 2 \mathrm{~B}-\mathrm{C} 3 \mathrm{~B}-\mathrm{C} 4 \mathrm{~B}$ & $1.0(3)$ \\
\hline $\mathrm{N} 4 \mathrm{~A}-\mathrm{C} 4 \mathrm{~A}-\mathrm{C} 5 \mathrm{~A}-\mathrm{C} 6 \mathrm{~A}$ & $-177.58(15)$ & $\mathrm{C} 2 \mathrm{~B}-\mathrm{C} 3 \mathrm{~B}-\mathrm{C} 4 \mathrm{~B}-\mathrm{C} 5 \mathrm{~B}$ & $-0.1(3)$ \\
\hline $\mathrm{N} 3 \mathrm{~A}-\mathrm{C} 4 \mathrm{~A}-\mathrm{C} 5 \mathrm{~A}-\mathrm{C} 6 \mathrm{~A}$ & $3.1(2)$ & $\mathrm{C} 3 \mathrm{~B}-\mathrm{C} 4 \mathrm{~B}-\mathrm{C} 5 \mathrm{~B}-\mathrm{C} 6 \mathrm{~B}$ & $-0.9(3)$ \\
\hline
\end{tabular}




$\begin{array}{llll}\mathrm{N} 4 \mathrm{~A}-\mathrm{C} 4 \mathrm{~A}-\mathrm{C} 5 \mathrm{~A}-\mathrm{C} 11 & 3.2(2) & \mathrm{C} 4 \mathrm{~B}-\mathrm{C} 5 \mathrm{~B}-\mathrm{C} 6 \mathrm{~B}-\mathrm{C} 1 \mathrm{~B} & 1.1(3) \\ \mathrm{N} 3 \mathrm{~A}-\mathrm{C} 4 \mathrm{~A}-\mathrm{C} 5 \mathrm{~A}-\mathrm{C} 1 & -176.17(12) & \mathrm{C} 2 \mathrm{~B}-\mathrm{C} 1 \mathrm{~B}-\mathrm{C} 6 \mathrm{~B}-\mathrm{C} 5 \mathrm{~B} & -0.3(3) \\ \mathrm{C} 2 \mathrm{~A}-\mathrm{N} 1 \mathrm{~A}-\mathrm{C} 6 \mathrm{~A}-\mathrm{C} 5 \mathrm{~A} & -2.0(2) & \mathrm{C} 7 \mathrm{~B}-\mathrm{C} 1 \mathrm{~B}-\mathrm{C} 6 \mathrm{~B}-\mathrm{C} 5 \mathrm{~B} & -178.55(16) \\ \mathrm{C} 2 \mathrm{~A}-\mathrm{N} 1 \mathrm{~A}-\mathrm{C} 6 \mathrm{~A}-\mathrm{C} 8 \mathrm{~A} & 178.93(14) & \mathrm{C} 6 \mathrm{~B}-\mathrm{C} 1 \mathrm{~B}-\mathrm{C} 7 \mathrm{~B}-\mathrm{O} 2 \mathrm{~B} & -179.88(16) \\ \mathrm{C} 4 \mathrm{~A}-\mathrm{C} 5 \mathrm{~A}-\mathrm{C} 6 \mathrm{~A}-\mathrm{N} 1 \mathrm{~A} & -0.5(2) & \mathrm{C} 2 \mathrm{~B}-\mathrm{C} 1 \mathrm{~B}-\mathrm{C} 7 \mathrm{~B}-\mathrm{O} 2 \mathrm{~B} & 1.9(3) \\ \mathrm{C} 11-\mathrm{C} 5 \mathrm{~A}-\mathrm{C} 6 \mathrm{~A}-\mathrm{N} 1 \mathrm{~A} & 178.68(12) & \mathrm{C} 6 \mathrm{~B}-\mathrm{C} 1 \mathrm{~B}-\mathrm{C} 7 \mathrm{~B}-\mathrm{O} 1 \mathrm{~B} & 0.5(2) \\ \mathrm{C} 4 \mathrm{~A}-\mathrm{C} 5 \mathrm{~A}-\mathrm{C} 6 \mathrm{~A}-\mathrm{C} 8 \mathrm{~A} & 178.53(14) & \mathrm{C} 2 \mathrm{~B}-\mathrm{C} 1 \mathrm{~B}-\mathrm{C} 7 \mathrm{~B}-\mathrm{O} 1 \mathrm{~B} & -177.71(15) \\ \mathrm{C} 11-\mathrm{C} 5 \mathrm{~A}-\mathrm{C} 6 \mathrm{~A}-\mathrm{C} 8 \mathrm{~A} & -2.3(2) & & \end{array}$

Hydrogen-bond geometry $\left(\AA,{ }^{\circ}\right)$

\begin{tabular}{lllll}
\hline$D-\mathrm{H} \cdots A$ & $D-\mathrm{H}$ & $\mathrm{H} \cdots A$ & $D \cdots A$ & $D-\mathrm{H} \cdots A$ \\
\hline $\mathrm{N} 4 A-\mathrm{H} 4 A 1 \cdots \mathrm{N} 3 A^{\mathrm{i}}$ & $0.89(3)$ & $2.14(3)$ & $3.027(2)$ & $176(2)$ \\
$\mathrm{N} 4 A-\mathrm{H} 4 A 2 \cdots \mathrm{O} 2 B^{\mathrm{ii}}$ & $0.87(3)$ & $2.23(3)$ & $3.034(2)$ & $154(2)$ \\
$\mathrm{C} 8 A-\mathrm{H} 8 A A \cdots \mathrm{O} 1 B$ & 0.98 & 2.62 & $3.402(2)$ & 137 \\
$\mathrm{O} 1 B-\mathrm{H} 1 B \cdots \mathrm{N} 1 A$ & $1.05(4)$ & $1.58(4)$ & $2.6235(19)$ & $170(3)$ \\
$\mathrm{N} 1 B-\mathrm{H} 1 B 1 \cdots \mathrm{O} 2 B$ & $0.91(3)$ & $2.04(3)$ & $2.722(2)$ & $131(2)$ \\
$\mathrm{N} 1 B-\mathrm{H} 1 B 2 \cdots \mathrm{O} 2 B^{\mathrm{iii}}$ & $0.84(3)$ & $2.27(3)$ & $3.100(2)$ & $171(2)$ \\
\hline
\end{tabular}

Symmetry codes: (i) $-x,-y+2,-z+1$; (ii) $-x,-y+1,-z+1$; (iii) $-x+1 / 2, y-1 / 2,-z+3 / 2$.

4-Amino-5-chloro-2,6-dimethylpyrimidine-5-methylthiophene-2-carboxylic acid (1/1) (COCRYSTAL-5)

Crystal data

$\mathrm{C}_{6} \mathrm{H}_{8} \mathrm{ClN}_{3} \cdot \mathrm{C}_{6} \mathrm{H}_{6} \mathrm{O}_{2} \mathrm{~S}$

$M_{r}=299.77$

Monoclinic, $P 2_{1} / c$

$a=8.273(3) \AA$

$b=12.746(4) \AA$

$c=13.320(4) \AA$

$\beta=102.390(5)^{\circ}$

$V=1371.8(8) \AA^{3}$

$Z=4$

\section{Data collection}

\section{Bruker APEXII CCD}

diffractometer

Radiation source: fine focus sealed tube

Graphite monochromator

$\omega$ and phi scans

Absorption correction: multi-scan

(APEX2; Bruker, 2014)

$T_{\min }=0.608, T_{\max }=0.746$

\section{Refinement}

Refinement on $F^{2}$

Least-squares matrix: full

$R\left[F^{2}>2 \sigma\left(F^{2}\right)\right]=0.034$

$w R\left(F^{2}\right)=0.092$

$S=1.05$

4109 reflections

189 parameters

0 restraints
$F(000)=624$

$D_{\mathrm{x}}=1.452 \mathrm{Mg} \mathrm{m}^{-3}$

Mo $K \alpha$ radiation, $\lambda=0.71073 \AA$

Cell parameters from 5891 reflections

$\theta=2.2-31.4^{\circ}$

$\mu=0.43 \mathrm{~mm}^{-1}$

$T=100 \mathrm{~K}$

Plate, colourless

$0.55 \times 0.50 \times 0.30 \mathrm{~mm}$

8807 measured reflections

4109 independent reflections

3735 reflections with $I>2 \sigma(I)$

$R_{\text {int }}=0.028$

$\theta_{\text {max }}=31.4^{\circ}, \theta_{\min }=2.2^{\circ}$

$h=-11 \rightarrow 12$

$k=-17 \rightarrow 17$

$l=-11 \rightarrow 18$

Primary atom site location: structure-invariant direct methods

Secondary atom site location: difference Fourier map

Hydrogen site location: mixed

$\mathrm{H}$ atoms treated by a mixture of independent and constrained refinement 
$w=1 /\left[\sigma^{2}\left(F_{\mathrm{o}}^{2}\right)+(0.0473 P)^{2}+0.5783 P\right]$

where $P=\left(F_{\mathrm{o}}^{2}+2 F_{\mathrm{c}}^{2}\right) / 3$

$(\Delta / \sigma)_{\max }=0.001$

$$
\Delta \rho_{\max }=0.55 \text { e } \AA^{-3}
$$

$\Delta \rho_{\min }=-0.29$ e $\AA^{-3}$

\section{Special details}

Geometry. All esds (except the esd in the dihedral angle between two 1.s. planes) are estimated using the full covariance matrix. The cell esds are taken into account individually in the estimation of esds in distances, angles and torsion angles; correlations between esds in cell parameters are only used when they are defined by crystal symmetry. An approximate (isotropic) treatment of cell esds is used for estimating esds involving l.s. planes.

Fractional atomic coordinates and isotropic or equivalent isotropic displacement parameters $\left(\hat{A}^{2}\right)$

\begin{tabular}{|c|c|c|c|c|c|}
\hline & $x$ & $y$ & $z$ & $U_{\text {iso }} * / U_{\text {eq }}$ & Occ. $(<1)$ \\
\hline $\mathrm{Cl1}$ & $0.38618(3)$ & $0.84503(2)$ & $0.35758(2)$ & $0.01547(8)$ & \\
\hline N1A & $0.54569(12)$ & $0.74068(7)$ & $0.64354(7)$ & $0.01340(18)$ & \\
\hline $\mathrm{C} 2 \mathrm{~A}$ & $0.62308(14)$ & $0.65402(8)$ & $0.62325(8)$ & $0.0128(2)$ & \\
\hline N3A & $0.63936(12)$ & $0.62204(7)$ & $0.52992(7)$ & $0.01266(18)$ & \\
\hline N4A & $0.59228(14)$ & $0.65222(8)$ & $0.35501(8)$ & $0.0163(2)$ & \\
\hline H41 & $0.560(2)$ & $0.6922(15)$ & $0.3027(15)$ & $0.032(5)^{*}$ & \\
\hline $\mathrm{H} 42$ & $0.647(2)$ & $0.5971(15)$ & $0.3525(14)$ & $0.026(4)^{*}$ & \\
\hline $\mathrm{C} 4 \mathrm{~A}$ & $0.57179(13)$ & $0.68167(8)$ & $0.44758(8)$ & 0.01177 (19) & \\
\hline C5A & $0.48186(13)$ & $0.77260(8)$ & $0.46351(8)$ & $0.01156(19)$ & \\
\hline C6A & $0.47182(13)$ & $0.80027(8)$ & $0.56179(8)$ & 0.01207 (19) & \\
\hline C7A & $0.69907(16)$ & $0.58473(10)$ & $0.71194(9)$ & $0.0190(2)$ & \\
\hline H7A1 & 0.7278 & 0.5169 & 0.6859 & $0.028 *$ & $0.282(19)$ \\
\hline H7A2 & 0.6198 & 0.5740 & 0.7561 & $0.028 *$ & $0.282(19)$ \\
\hline H7A3 & 0.7992 & 0.6181 & 0.7518 & $0.028 *$ & $0.282(19)$ \\
\hline H7A4 & 0.7034 & 0.6224 & 0.7766 & $0.028 *$ & $0.718(19)$ \\
\hline H7A5 & 0.8115 & 0.5653 & 0.7064 & $0.028 *$ & $0.718(19)$ \\
\hline H7A6 & 0.6320 & 0.5212 & 0.7107 & $0.028 *$ & $0.718(19)$ \\
\hline $\mathrm{C} 8 \mathrm{~A}$ & $0.38111(15)$ & $0.89640(9)$ & $0.58452(9)$ & $0.0167(2)$ & \\
\hline H8A1 & 0.3804 & 0.8984 & 0.6580 & $0.025 *$ & $0.717(18)$ \\
\hline H8A2 & 0.2670 & 0.8944 & 0.5444 & $0.025^{*}$ & $0.717(18)$ \\
\hline H8A3 & 0.4367 & 0.9591 & 0.5660 & $0.025^{*}$ & $0.717(18)$ \\
\hline H8A4 & 0.3424 & 0.9362 & 0.5210 & $0.025 *$ & $0.283(18)$ \\
\hline H8A5 & 0.4557 & 0.9403 & 0.6345 & $0.025 *$ & $0.283(18)$ \\
\hline H8A6 & 0.2860 & 0.8755 & 0.6129 & $0.025 *$ & $0.283(18)$ \\
\hline $\mathrm{S} 1$ & $0.94879(4)$ & $0.25627(2)$ & $0.33202(2)$ & $0.01614(8)$ & \\
\hline O1B & $0.81492(12)$ & $0.44704(7)$ & $0.53060(7)$ & 0.02158 (19) & \\
\hline H1B & $0.752(3)$ & $0.5071(18)$ & $0.5248(16)$ & $0.044(6)^{*}$ & \\
\hline $\mathrm{O} 2 \mathrm{~B}$ & $0.76792(12)$ & $0.45470(7)$ & $0.35803(7)$ & $0.02004(18)$ & \\
\hline $\mathrm{C} 1 \mathrm{~B}$ & $0.82798(14)$ & $0.41179(9)$ & $0.43965(9)$ & $0.0150(2)$ & \\
\hline $\mathrm{C} 2 \mathrm{~B}$ & $0.92271(14)$ & $0.31337(9)$ & $0.44446(9)$ & $0.0145(2)$ & \\
\hline C3B & $0.99609(16)$ & $0.25478(9)$ & $0.52815(10)$ & $0.0185(2)$ & \\
\hline H3BA & 0.9950 & 0.2734 & 0.5971 & $0.022 *$ & \\
\hline $\mathrm{C} 4 \mathrm{~B}$ & $1.07400(16)$ & $0.16312(10)$ & $0.50029(10)$ & $0.0200(2)$ & \\
\hline H4BA & 1.1305 & 0.1137 & 0.5488 & $0.024 *$ & \\
\hline C5B & $1.05905(15)$ & $0.15343(9)$ & $0.39617(10)$ & $0.0162(2)$ & \\
\hline C6B & $1.12401(17)$ & $0.06825(10)$ & $0.33793(11)$ & $0.0232(3)$ & \\
\hline
\end{tabular}




\begin{tabular}{lllll} 
H6BA & 1.1161 & 0.0007 & 0.3716 & $0.035^{*}$ \\
H6BB & 1.2400 & 0.0826 & 0.3368 & $0.035^{*}$ \\
H6BC & 1.0584 & 0.0658 & 0.2673 & $0.035^{*}$ \\
\hline
\end{tabular}

Atomic displacement parameters $\left(\AA^{2}\right)$

\begin{tabular}{lllllll}
\hline & $U^{11}$ & $U^{22}$ & $U^{33}$ & $U^{12}$ & $U^{13}$ & $U^{23}$ \\
\hline C11 & $0.01899(14)$ & $0.01426(13)$ & $0.01211(13)$ & $0.00442(9)$ & $0.00102(10)$ & $0.00099(8)$ \\
N1A & $0.0154(4)$ & $0.0144(4)$ & $0.0109(4)$ & $-0.0005(3)$ & $0.0039(3)$ & $-0.0008(3)$ \\
C2A & $0.0143(5)$ & $0.0139(5)$ & $0.0105(5)$ & $-0.0008(4)$ & $0.0032(4)$ & $0.0004(3)$ \\
N3A & $0.0151(4)$ & $0.0126(4)$ & $0.0104(4)$ & $0.0015(3)$ & $0.0029(3)$ & $0.0003(3)$ \\
N4A & $0.0248(5)$ & $0.0146(4)$ & $0.0096(4)$ & $0.0071(4)$ & $0.0039(4)$ & $0.0000(3)$ \\
C4A & $0.0134(5)$ & $0.0116(4)$ & $0.0103(5)$ & $-0.0005(4)$ & $0.0026(4)$ & $-0.0011(4)$ \\
C5A & $0.0124(5)$ & $0.0113(4)$ & $0.0105(4)$ & $0.0007(3)$ & $0.0014(4)$ & $0.0003(3)$ \\
C6A & $0.0120(5)$ & $0.0120(4)$ & $0.0126(5)$ & $-0.0014(4)$ & $0.0034(4)$ & $-0.0020(4)$ \\
C7A & $0.0246(6)$ & $0.0203(5)$ & $0.0121(5)$ & $0.0044(4)$ & $0.0040(4)$ & $0.0040(4)$ \\
C8A & $0.0189(5)$ & $0.0149(5)$ & $0.0173(5)$ & $0.0025(4)$ & $0.0062(4)$ & $-0.0032(4)$ \\
S1 & $0.01864(15)$ & $0.01600(14)$ & $0.01424(14)$ & $0.00445(9)$ & $0.00458(11)$ & $0.00057(9)$ \\
O1B & $0.0302(5)$ & $0.0194(4)$ & $0.0160(4)$ & $0.0103(4)$ & $0.0069(4)$ & $-0.0002(3)$ \\
O2B & $0.0276(5)$ & $0.0161(4)$ & $0.0166(4)$ & $0.0066(3)$ & $0.0050(3)$ & $0.0004(3)$ \\
C1B & $0.0156(5)$ & $0.0128(4)$ & $0.0175(5)$ & $0.0005(4)$ & $0.0053(4)$ & $-0.0010(4)$ \\
C2B & $0.0149(5)$ & $0.0140(5)$ & $0.0154(5)$ & $0.0015(4)$ & $0.0045(4)$ & $-0.0008(4)$ \\
C3B & $0.0204(6)$ & $0.0197(5)$ & $0.0149(5)$ & $0.0038(4)$ & $0.0027(4)$ & $0.0001(4)$ \\
C4B & $0.0203(6)$ & $0.0186(5)$ & $0.0197(6)$ & $0.0067(4)$ & $0.0014(4)$ & $0.0033(4)$ \\
C5B & $0.0136(5)$ & $0.0144(5)$ & $0.0205(5)$ & $0.0028(4)$ & $0.0034(4)$ & $0.0001(4)$ \\
C6B & $0.0210(6)$ & $0.0191(5)$ & $0.0305(7)$ & $0.0051(5)$ & $0.0078(5)$ & $-0.0050(5)$ \\
& & & & & &
\end{tabular}

Geometric parameters $\left(\AA,{ }^{\circ}\right)$

\begin{tabular}{llll}
\hline C11-C5A & $1.7293(11)$ & C8A-H8A3 & 0.9800 \\
N1A-C2A & $1.3331(14)$ & C8A-H8A4 & 0.9800 \\
N1A-C6A & $1.3603(15)$ & C8A-H8A5 & 0.9800 \\
C2A-N3A & $1.3421(14)$ & C8A-H8A6 & 0.9800 \\
C2A-C7A & $1.5007(16)$ & S1-C5B & $1.7163(12)$ \\
N3A-C4A & $1.3528(14)$ & S1-C2B & $1.7199(12)$ \\
N4A-C4A & $1.3340(14)$ & O1B-C1B & $1.3180(15)$ \\
N4A-H41 & $0.86(2)$ & O1B-H1B & $0.92(2)$ \\
N4A-H42 & $0.842(19)$ & O2B-C1B & $1.2232(15)$ \\
C4A-C5A & $1.4175(15)$ & C1B-C2B & $1.4732(16)$ \\
C5A-C6A & $1.3753(15)$ & C2B-C3B & $1.3706(16)$ \\
C6A-C8A & $1.5013(15)$ & C3B-C4B & $1.4215(17)$ \\
C7A-H7A1 & 0.9800 & C3B-H3BA & 0.9500 \\
C7A-H7A2 & 0.9800 & C4B-C5B & $1.3712(18)$ \\
C7A-H7A3 & 0.9800 & C4B-H4BA & 0.9500 \\
C7A-H7A4 & 0.9800 & C5B-C6B & $1.4994(17)$ \\
C7A-H7A5 & 0.9800 & C6B-H6BA & 0.9800 \\
C7A-H7A6 & 0.9800 & C6B-H6BB & 0.9800 \\
C8A-H8A1 & 0.9800 & C6B-H6BC & 0.9800
\end{tabular}




\begin{tabular}{|c|c|}
\hline $\mathrm{C} 8 \mathrm{~A}-\mathrm{H} 8 \mathrm{~A} 2$ & 0.9800 \\
\hline $\mathrm{C} 2 \mathrm{~A}-\mathrm{N} 1 \mathrm{~A}-\mathrm{C} 6 \mathrm{~A}$ & $116.94(10)$ \\
\hline $\mathrm{N} 1 \mathrm{~A}-\mathrm{C} 2 \mathrm{~A}-\mathrm{N} 3 \mathrm{~A}$ & $125.97(10)$ \\
\hline $\mathrm{N} 1 \mathrm{~A}-\mathrm{C} 2 \mathrm{~A}-\mathrm{C} 7 \mathrm{~A}$ & $117.79(10)$ \\
\hline $\mathrm{N} 3 \mathrm{~A}-\mathrm{C} 2 \mathrm{~A}-\mathrm{C} 7 \mathrm{~A}$ & $116.23(10)$ \\
\hline $\mathrm{C} 2 \mathrm{~A}-\mathrm{N} 3 \mathrm{~A}-\mathrm{C} 4 \mathrm{~A}$ & $118.30(9)$ \\
\hline $\mathrm{C} 4 \mathrm{~A}-\mathrm{N} 4 \mathrm{~A}-\mathrm{H} 41$ & $120.2(13)$ \\
\hline $\mathrm{C} 4 \mathrm{~A}-\mathrm{N} 4 \mathrm{~A}-\mathrm{H} 42$ & $116.7(12)$ \\
\hline $\mathrm{H} 41-\mathrm{N} 4 \mathrm{~A}-\mathrm{H} 42$ & $122.8(18)$ \\
\hline $\mathrm{N} 4 \mathrm{~A}-\mathrm{C} 4 \mathrm{~A}-\mathrm{N} 3 \mathrm{~A}$ & $118.61(10)$ \\
\hline $\mathrm{N} 4 \mathrm{~A}-\mathrm{C} 4 \mathrm{~A}-\mathrm{C} 5 \mathrm{~A}$ & $122.91(10)$ \\
\hline $\mathrm{N} 3 \mathrm{~A}-\mathrm{C} 4 \mathrm{~A}-\mathrm{C} 5 \mathrm{~A}$ & $118.48(10)$ \\
\hline $\mathrm{C} 6 \mathrm{~A}-\mathrm{C} 5 \mathrm{~A}-\mathrm{C} 4 \mathrm{~A}$ & $119.53(10)$ \\
\hline $\mathrm{C} 6 \mathrm{~A}-\mathrm{C} 5 \mathrm{~A}-\mathrm{Cl1}$ & $121.95(9)$ \\
\hline $\mathrm{C} 4 \mathrm{~A}-\mathrm{C} 5 \mathrm{~A}-\mathrm{C} 11$ & $118.52(8)$ \\
\hline $\mathrm{N} 1 \mathrm{~A}-\mathrm{C} 6 \mathrm{~A}-\mathrm{C} 5 \mathrm{~A}$ & $120.68(10)$ \\
\hline $\mathrm{N} 1 \mathrm{~A}-\mathrm{C} 6 \mathrm{~A}-\mathrm{C} 8 \mathrm{~A}$ & $116.90(10)$ \\
\hline $\mathrm{C} 5 \mathrm{~A}-\mathrm{C} 6 \mathrm{~A}-\mathrm{C} 8 \mathrm{~A}$ & $122.42(10)$ \\
\hline $\mathrm{C} 2 \mathrm{~A}-\mathrm{C} 7 \mathrm{~A}-\mathrm{H} 7 \mathrm{~A} 1$ & 109.5 \\
\hline $\mathrm{C} 2 \mathrm{~A}-\mathrm{C} 7 \mathrm{~A}-\mathrm{H} 7 \mathrm{~A} 2$ & 109.5 \\
\hline $\mathrm{H} 7 \mathrm{~A} 1-\mathrm{C} 7 \mathrm{~A}-\mathrm{H} 7 \mathrm{~A} 2$ & 109.5 \\
\hline $\mathrm{C} 2 \mathrm{~A}-\mathrm{C} 7 \mathrm{~A}-\mathrm{H} 7 \mathrm{~A} 3$ & 109.5 \\
\hline $\mathrm{H} 7 \mathrm{~A} 1-\mathrm{C} 7 \mathrm{~A}-\mathrm{H} 7 \mathrm{~A} 3$ & 109.5 \\
\hline $\mathrm{H} 7 \mathrm{~A} 2-\mathrm{C} 7 \mathrm{~A}-\mathrm{H} 7 \mathrm{~A} 3$ & 109.5 \\
\hline $\mathrm{C} 2 \mathrm{~A}-\mathrm{C} 7 \mathrm{~A}-\mathrm{H} 7 \mathrm{~A} 4$ & 109.5 \\
\hline H7A1-C7A-H7A4 & 141.1 \\
\hline $\mathrm{H} 7 \mathrm{~A} 2-\mathrm{C} 7 \mathrm{~A}-\mathrm{H} 7 \mathrm{~A} 4$ & 56.3 \\
\hline $\mathrm{H} 7 \mathrm{~A} 3-\mathrm{C} 7 \mathrm{~A}-\mathrm{H} 7 \mathrm{~A} 4$ & 56.3 \\
\hline $\mathrm{C} 2 \mathrm{~A}-\mathrm{C} 7 \mathrm{~A}-\mathrm{H} 7 \mathrm{~A} 5$ & 109.5 \\
\hline $\mathrm{H} 7 \mathrm{~A} 1-\mathrm{C} 7 \mathrm{~A}-\mathrm{H} 7 \mathrm{~A} 5$ & 56.3 \\
\hline $\mathrm{H} 7 \mathrm{~A} 2-\mathrm{C} 7 \mathrm{~A}-\mathrm{H} 7 \mathrm{~A} 5$ & 141.1 \\
\hline H7A3-C7A-H7A5 & 56.3 \\
\hline H7A4-C7A-H7A5 & 109.5 \\
\hline $\mathrm{C} 2 \mathrm{~A}-\mathrm{C} 7 \mathrm{~A}-\mathrm{H} 7 \mathrm{~A} 6$ & 109.5 \\
\hline $\mathrm{H} 7 \mathrm{~A} 1-\mathrm{C} 7 \mathrm{~A}-\mathrm{H} 7 \mathrm{~A} 6$ & 56.3 \\
\hline $\mathrm{H} 7 \mathrm{~A} 2-\mathrm{C} 7 \mathrm{~A}-\mathrm{H} 7 \mathrm{~A} 6$ & 56.3 \\
\hline H7A3-C7A-H7A6 & 141.1 \\
\hline $\mathrm{H} 7 \mathrm{~A} 4-\mathrm{C} 7 \mathrm{~A}-\mathrm{H} 7 \mathrm{~A} 6$ & 109.5 \\
\hline H7A5-C7A-H7A6 & 109.5 \\
\hline $\mathrm{C} 6 \mathrm{~A}-\mathrm{C} 8 \mathrm{~A}-\mathrm{H} 8 \mathrm{~A} 1$ & 109.5 \\
\hline $\mathrm{C} 6 \mathrm{~A}-\mathrm{C} 8 \mathrm{~A}-\mathrm{H} 8 \mathrm{~A} 2$ & 109.5 \\
\hline $\mathrm{H} 8 \mathrm{~A} 1-\mathrm{C} 8 \mathrm{~A}-\mathrm{H} 8 \mathrm{~A} 2$ & 109.5 \\
\hline $\mathrm{C} 6 \mathrm{~A}-\mathrm{N} 1 \mathrm{~A}-\mathrm{C} 2 \mathrm{~A}-\mathrm{N} 3 \mathrm{~A}$ & $2.31(17)$ \\
\hline $\mathrm{C} 6 \mathrm{~A}-\mathrm{N} 1 \mathrm{~A}-\mathrm{C} 2 \mathrm{~A}-\mathrm{C} 7 \mathrm{~A}$ & $-177.41(10)$ \\
\hline $\mathrm{N} 1 \mathrm{~A}-\mathrm{C} 2 \mathrm{~A}-\mathrm{N} 3 \mathrm{~A}-\mathrm{C} 4 \mathrm{~A}$ & $-0.18(17)$ \\
\hline $\mathrm{C} 7 \mathrm{~A}-\mathrm{C} 2 \mathrm{~A}-\mathrm{N} 3 \mathrm{~A}-\mathrm{C} 4 \mathrm{~A}$ & $179.55(10)$ \\
\hline
\end{tabular}

\begin{tabular}{|c|c|}
\hline $\mathrm{C} 6 \mathrm{~A}-\mathrm{C} 8 \mathrm{~A}-\mathrm{H} 8 \mathrm{~A} 3$ & 109.5 \\
\hline $\mathrm{H} 8 \mathrm{~A} 1-\mathrm{C} 8 \mathrm{~A}-\mathrm{H} 8 \mathrm{~A} 3$ & 109.5 \\
\hline $\mathrm{H} 8 \mathrm{~A} 2-\mathrm{C} 8 \mathrm{~A}-\mathrm{H} 8 \mathrm{~A} 3$ & 109.5 \\
\hline $\mathrm{C} 6 \mathrm{~A}-\mathrm{C} 8 \mathrm{~A}-\mathrm{H} 8 \mathrm{~A} 4$ & 109.5 \\
\hline $\mathrm{H} 8 \mathrm{~A} 1-\mathrm{C} 8 \mathrm{~A}-\mathrm{H} 8 \mathrm{~A} 4$ & 141.1 \\
\hline $\mathrm{H} 8 \mathrm{~A} 2-\mathrm{C} 8 \mathrm{~A}-\mathrm{H} 8 \mathrm{~A} 4$ & 56.3 \\
\hline $\mathrm{H} 8 \mathrm{~A} 3-\mathrm{C} 8 \mathrm{~A}-\mathrm{H} 8 \mathrm{~A} 4$ & 56.3 \\
\hline $\mathrm{C} 6 \mathrm{~A}-\mathrm{C} 8 \mathrm{~A}-\mathrm{H} 8 \mathrm{~A} 5$ & 109.5 \\
\hline $\mathrm{H} 8 \mathrm{~A} 1-\mathrm{C} 8 \mathrm{~A}-\mathrm{H} 8 \mathrm{~A} 5$ & 56.3 \\
\hline $\mathrm{H} 8 \mathrm{~A} 2-\mathrm{C} 8 \mathrm{~A}-\mathrm{H} 8 \mathrm{~A} 5$ & 141.1 \\
\hline $\mathrm{H} 8 \mathrm{~A} 3-\mathrm{C} 8 \mathrm{~A}-\mathrm{H} 8 \mathrm{~A} 5$ & 56.3 \\
\hline $\mathrm{H} 8 \mathrm{~A} 4-\mathrm{C} 8 \mathrm{~A}-\mathrm{H} 8 \mathrm{~A} 5$ & 109.5 \\
\hline $\mathrm{C} 6 \mathrm{~A}-\mathrm{C} 8 \mathrm{~A}-\mathrm{H} 8 \mathrm{~A} 6$ & 109.5 \\
\hline $\mathrm{H} 8 \mathrm{~A} 1-\mathrm{C} 8 \mathrm{~A}-\mathrm{H} 8 \mathrm{~A} 6$ & 56.3 \\
\hline $\mathrm{H} 8 \mathrm{~A} 2-\mathrm{C} 8 \mathrm{~A}-\mathrm{H} 8 \mathrm{~A} 6$ & 56.3 \\
\hline $\mathrm{H} 8 \mathrm{~A} 3-\mathrm{C} 8 \mathrm{~A}-\mathrm{H} 8 \mathrm{~A} 6$ & 141.1 \\
\hline $\mathrm{H} 8 \mathrm{~A} 4-\mathrm{C} 8 \mathrm{~A}-\mathrm{H} 8 \mathrm{~A} 6$ & 109.5 \\
\hline $\mathrm{H} 8 \mathrm{~A} 5-\mathrm{C} 8 \mathrm{~A}-\mathrm{H} 8 \mathrm{~A} 6$ & 109.5 \\
\hline $\mathrm{C} 5 \mathrm{~B}-\mathrm{S} 1-\mathrm{C} 2 \mathrm{~B}$ & $92.42(6)$ \\
\hline $\mathrm{C} 1 \mathrm{~B}-\mathrm{O} 1 \mathrm{~B}-\mathrm{H} 1 \mathrm{~B}$ & $111.3(13)$ \\
\hline $\mathrm{O} 2 \mathrm{~B}-\mathrm{C} 1 \mathrm{~B}-\mathrm{O} 1 \mathrm{~B}$ & $124.53(11)$ \\
\hline $\mathrm{O} 2 \mathrm{~B}-\mathrm{C} 1 \mathrm{~B}-\mathrm{C} 2 \mathrm{~B}$ & $122.02(11)$ \\
\hline $\mathrm{O} 1 \mathrm{~B}-\mathrm{C} 1 \mathrm{~B}-\mathrm{C} 2 \mathrm{~B}$ & $113.46(10)$ \\
\hline $\mathrm{C} 3 \mathrm{~B}-\mathrm{C} 2 \mathrm{~B}-\mathrm{C} 1 \mathrm{~B}$ & $129.64(11)$ \\
\hline $\mathrm{C} 3 \mathrm{~B}-\mathrm{C} 2 \mathrm{~B}-\mathrm{S} 1$ & $111.17(9)$ \\
\hline $\mathrm{C} 1 \mathrm{~B}-\mathrm{C} 2 \mathrm{~B}-\mathrm{S} 1$ & $119.18(9)$ \\
\hline $\mathrm{C} 2 \mathrm{~B}-\mathrm{C} 3 \mathrm{~B}-\mathrm{C} 4 \mathrm{~B}$ & $112.46(11)$ \\
\hline $\mathrm{C} 2 \mathrm{~B}-\mathrm{C} 3 \mathrm{~B}-\mathrm{H} 3 \mathrm{BA}$ & 123.8 \\
\hline $\mathrm{C} 4 \mathrm{~B}-\mathrm{C} 3 \mathrm{~B}-\mathrm{H} 3 \mathrm{BA}$ & 123.8 \\
\hline $\mathrm{C} 5 \mathrm{~B}-\mathrm{C} 4 \mathrm{~B}-\mathrm{C} 3 \mathrm{~B}$ & $112.98(11)$ \\
\hline $\mathrm{C} 5 \mathrm{~B}-\mathrm{C} 4 \mathrm{~B}-\mathrm{H} 4 \mathrm{BA}$ & 123.5 \\
\hline $\mathrm{C} 3 \mathrm{~B}-\mathrm{C} 4 \mathrm{~B}-\mathrm{H} 4 \mathrm{BA}$ & 123.5 \\
\hline $\mathrm{C} 4 \mathrm{~B}-\mathrm{C} 5 \mathrm{~B}-\mathrm{C} 6 \mathrm{~B}$ & $128.60(11)$ \\
\hline $\mathrm{C} 4 \mathrm{~B}-\mathrm{C} 5 \mathrm{~B}-\mathrm{S} 1$ & $110.96(9)$ \\
\hline $\mathrm{C} 6 \mathrm{~B}-\mathrm{C} 5 \mathrm{~B}-\mathrm{S} 1$ & $120.44(10)$ \\
\hline $\mathrm{C} 5 \mathrm{~B}-\mathrm{C} 6 \mathrm{~B}-\mathrm{H} 6 \mathrm{BA}$ & 109.5 \\
\hline $\mathrm{C} 5 \mathrm{~B}-\mathrm{C} 6 \mathrm{~B}-\mathrm{H} 6 \mathrm{BB}$ & 109.5 \\
\hline $\mathrm{H} 6 \mathrm{BA}-\mathrm{C} 6 \mathrm{~B}-\mathrm{H} 6 \mathrm{BB}$ & 109.5 \\
\hline $\mathrm{C} 5 \mathrm{~B}-\mathrm{C} 6 \mathrm{~B}-\mathrm{H} 6 \mathrm{BC}$ & 109.5 \\
\hline $\mathrm{H} 6 \mathrm{BA}-\mathrm{C} 6 \mathrm{~B}-\mathrm{H} 6 \mathrm{BC}$ & 109.5 \\
\hline $\mathrm{H} 6 \mathrm{BB}-\mathrm{C} 6 \mathrm{~B}-\mathrm{H} 6 \mathrm{BC}$ & 109.5 \\
\hline $\mathrm{C} 11-\mathrm{C} 5 \mathrm{~A}-\mathrm{C} 6 \mathrm{~A}-\mathrm{C} 8 \mathrm{~A}$ & $-1.57(15)$ \\
\hline $\mathrm{O} 2 \mathrm{~B}-\mathrm{C} 1 \mathrm{~B}-\mathrm{C} 2 \mathrm{~B}-\mathrm{C} 3 \mathrm{~B}$ & $-179.21(12)$ \\
\hline $\mathrm{O} 1 \mathrm{~B}-\mathrm{C} 1 \mathrm{~B}-\mathrm{C} 2 \mathrm{~B}-\mathrm{C} 3 \mathrm{~B}$ & $0.46(18)$ \\
\hline $\mathrm{O} 2 \mathrm{~B}-\mathrm{C} 1 \mathrm{~B}-\mathrm{C} 2 \mathrm{~B}-\mathrm{S} 1$ & $-0.45(16)$ \\
\hline
\end{tabular}




$\begin{array}{llll}\mathrm{C} 2 \mathrm{~A}-\mathrm{N} 3 \mathrm{~A}-\mathrm{C} 4 \mathrm{~A}-\mathrm{N} 4 \mathrm{~A} & 177.78(10) & \mathrm{O} 1 \mathrm{~B}-\mathrm{C} 1 \mathrm{~B}-\mathrm{C} 2 \mathrm{~B}-\mathrm{S} 1 & 179.21(9) \\ \mathrm{C} 2 \mathrm{~A}-\mathrm{N} 3 \mathrm{~A}-\mathrm{C} 4 \mathrm{~A}-\mathrm{C} 5 \mathrm{~A} & -2.41(15) & \mathrm{C} 5 \mathrm{~B}-\mathrm{S} 1-\mathrm{C} 2 \mathrm{~B}-\mathrm{C} 3 \mathrm{~B} & -0.20(10) \\ \mathrm{N} 4 \mathrm{~A}-\mathrm{C} 4 \mathrm{~A}-\mathrm{C} 5 \mathrm{~A}-\mathrm{C} 6 \mathrm{~A} & -177.34(11) & \mathrm{C} 5 \mathrm{~B}-\mathrm{S} 1-\mathrm{C} 2 \mathrm{~B}-\mathrm{C} 1 \mathrm{~B} & -179.17(10) \\ \mathrm{N} 3 \mathrm{~A}-\mathrm{C} 4 \mathrm{~A}-\mathrm{C} 5 \mathrm{~A}-\mathrm{C} 6 \mathrm{~A} & 2.85(16) & \mathrm{C} 1 \mathrm{~B}-\mathrm{C} 2 \mathrm{~B}-\mathrm{C} 3 \mathrm{~B}-\mathrm{C} 4 \mathrm{~B} & 178.92(12) \\ \mathrm{N} 4 \mathrm{~A}-\mathrm{C} 4 \mathrm{~A}-\mathrm{C} 5 \mathrm{~A}-\mathrm{C} 1 & 3.19(15) & \mathrm{S} 1-\mathrm{C} 2 \mathrm{~B}-\mathrm{C} 3 \mathrm{~B}-\mathrm{C} 4 \mathrm{~B} & 0.09(14) \\ \mathrm{N} 3 \mathrm{~A}-\mathrm{C} 4 \mathrm{~A}-\mathrm{C} 5 \mathrm{~A}-\mathrm{C} 1 & -176.62(8) & \mathrm{C} 2 \mathrm{~B}-\mathrm{C} 3 \mathrm{~B}-\mathrm{C} 4 \mathrm{~B}-\mathrm{C} 5 \mathrm{~B} & 0.11(17) \\ \mathrm{C} 2 \mathrm{~A}-\mathrm{N} 1 \mathrm{~A}-\mathrm{C} 6 \mathrm{~A}-\mathrm{C} 5 \mathrm{~A} & -1.75(16) & \mathrm{C} 3 \mathrm{~B}-\mathrm{C} 4 \mathrm{~B}-\mathrm{C} 5 \mathrm{~B}-\mathrm{C} 6 \mathrm{~B} & -179.99(12) \\ \mathrm{C} 2 \mathrm{~A}-\mathrm{N} 1 \mathrm{~A}-\mathrm{C} 6 \mathrm{~A}-\mathrm{C} 8 \mathrm{~A} & 178.53(10) & \mathrm{C} 3 \mathrm{~B}-\mathrm{C} 4 \mathrm{~B}-\mathrm{C} 5 \mathrm{~B}-\mathrm{S} 1 & -0.25(15) \\ \mathrm{C} 4 \mathrm{~A}-\mathrm{C} 5 \mathrm{~A}-\mathrm{C} 6 \mathrm{~A}-\mathrm{N} 1 \mathrm{~A} & -0.72(16) & \mathrm{C} 2 \mathrm{~B}-\mathrm{S} 1-\mathrm{C} 5 \mathrm{~B}-\mathrm{C} 4 \mathrm{~B} & 0.26(10) \\ \mathrm{C} 11-\mathrm{C} 5 \mathrm{~A}-\mathrm{C} 6 \mathrm{~A}-\mathrm{N} 1 \mathrm{~A} & 178.72(8) & \mathrm{C} 2 \mathrm{~B}-\mathrm{S} 1-\mathrm{C} 5 \mathrm{~B}-\mathrm{C} 6 \mathrm{~B} & -179.98(10) \\ \mathrm{C} 4 \mathrm{~A}-\mathrm{C} 5 \mathrm{~A}-\mathrm{C} 6 \mathrm{~A}-\mathrm{C} 8 \mathrm{~A} & 178.98(10) & & \end{array}$

Hydrogen-bond geometry $\left(\AA,{ }^{\circ}\right)$

\begin{tabular}{lllll}
\hline$D-\mathrm{H} \cdots A$ & $D-\mathrm{H}$ & $\mathrm{H} \cdots A$ & $D \cdots A$ & $D-\mathrm{H} \cdots A$ \\
\hline $\mathrm{N} 4 A-\mathrm{H} 41 \cdots \mathrm{N} 1 A^{\mathrm{i}}$ & $0.86(2)$ & $2.27(2)$ & $3.0790(16)$ & $158.0(18)$ \\
$\mathrm{N} 4 A-\mathrm{H} 42 \cdots \mathrm{O} 2 B$ & $0.842(19)$ & $2.065(19)$ & $2.9028(15)$ & $173.6(18)$ \\
$\mathrm{O} 1 B-\mathrm{H} 1 B \cdots \mathrm{N} 3 A$ & $0.92(2)$ & $1.74(2)$ & $2.6607(14)$ & $173(2)$ \\
\hline
\end{tabular}

Symmetry code: (i) $x,-y+3 / 2, z-1 / 2$.

4-Amino-5-chloro-2,6-dimethylpyrimidine-benzoic acid (1/1) (COCRYSTAL-6)

Crystal data

$\mathrm{C}_{6} \mathrm{H}_{8} \mathrm{ClN}_{3} \cdot \mathrm{C}_{7} \mathrm{H}_{6} \mathrm{O}_{2}$

$M_{r}=279.72$

Monoclinic, $P 2{ }_{1} / c$

$a=10.1421(5) \AA$

$b=10.4218(5) \AA$

$c=13.2681(6) \AA$

$\beta=107.804(5)^{\circ}$

$V=1335.26(12) \AA^{3}$

$Z=4$

\section{Data collection}

Agilent SuperNova Dual Source diffractometer with an Atlas detector

Radiation source: sealed X-ray tube Detector resolution: 10.6501 pixels $\mathrm{mm}^{-1}$ $\omega$ scans

Absorption correction: multi-scan

(CrysAlis PRO; Agilent, 2014)

$T_{\min }=0.763, T_{\max }=1.000$

\section{Refinement}

Refinement on $F^{2}$

Least-squares matrix: full

$R\left[F^{2}>2 \sigma\left(F^{2}\right)\right]=0.041$

$w R\left(F^{2}\right)=0.118$

$S=1.04$

2760 reflections

187 parameters

0 restraints
$F(000)=584$

$D_{\mathrm{x}}=1.391 \mathrm{Mg} \mathrm{m}^{-3}$

$\mathrm{Cu} K \alpha$ radiation, $\lambda=1.54184 \AA$

Cell parameters from 2966 reflections

$\theta=4.2-76.3^{\circ}$

$\mu=2.56 \mathrm{~mm}^{-1}$

$T=120 \mathrm{~K}$

Chunk, colorless

$0.52 \times 0.38 \times 0.24 \mathrm{~mm}$

6087 measured reflections

2760 independent reflections

2560 reflections with $I>2 \sigma(I)$

$R_{\text {int }}=0.029$

$\theta_{\text {max }}=76.6^{\circ}, \theta_{\min }=4.6^{\circ}$

$h=-12 \rightarrow 12$

$k=-9 \rightarrow 12$

$l=-16 \rightarrow 16$

Hydrogen site location: mixed

$\mathrm{H}$ atoms treated by a mixture of independent and constrained refinement

$w=1 /\left[\sigma^{2}\left(F_{\mathrm{o}}^{2}\right)+(0.0793 P)^{2}+0.3948 P\right]$ where $P=\left(F_{\mathrm{o}}^{2}+2 F_{\mathrm{c}}^{2}\right) / 3$

$(\Delta / \sigma)_{\max }<0.001$

$\Delta \rho_{\max }=0.33$ e $\AA^{-3}$

$\Delta \rho_{\min }=-0.44$ e $\AA^{-3}$ 
Extinction correction: SHELXL2014 (Sheldrick, 2015b),

$\mathrm{Fc}^{*}=\mathrm{kFc}\left[1+0.001 \mathrm{xFc}^{2} \lambda^{3} / \sin (2 \theta)\right]^{-1 / 4}$

Extinction coefficient: $0.0032(7)$

\section{Special details}

Geometry. All esds (except the esd in the dihedral angle between two 1.s. planes) are estimated using the full covariance matrix. The cell esds are taken into account individually in the estimation of esds in distances, angles and torsion angles; correlations between esds in cell parameters are only used when they are defined by crystal symmetry. An approximate (isotropic) treatment of cell esds is used for estimating esds involving l.s. planes.

Fractional atomic coordinates and isotropic or equivalent isotropic displacement parameters $\left(\AA^{2}\right)$

\begin{tabular}{|c|c|c|c|c|}
\hline & $x$ & $y$ & $z$ & $U_{\text {iso }} * / U_{\text {eq }}$ \\
\hline $\mathrm{Cl1}$ & $0.39577(4)$ & $0.14749(3)$ & $0.34668(3)$ & $0.02536(16)$ \\
\hline N1A & $0.54933(13)$ & 0.30904 (13) & $0.63034(9)$ & $0.0215(3)$ \\
\hline $\mathrm{C} 2 \mathrm{~A}$ & 0.63967 (14) & $0.38934(15)$ & $0.60876(11)$ & $0.0204(3)$ \\
\hline N3A & $0.66375(12)$ & $0.40143(12)$ & $0.51529(9)$ & $0.0201(3)$ \\
\hline N4A & $0.61835(15)$ & $0.33300(14)$ & $0.34212(10)$ & $0.0241(3)$ \\
\hline $\mathrm{H} 41$ & $0.682(2)$ & $0.386(2)$ & $0.3405(16)$ & $0.028(5)^{*}$ \\
\hline $\mathrm{H} 42$ & $0.583(2)$ & $0.273(2)$ & $0.2896(17)$ & $0.030(5)^{*}$ \\
\hline $\mathrm{C} 4 \mathrm{~A}$ & $0.59268(15)$ & $0.32453(14)$ & $0.43453(11)$ & $0.0196(3)$ \\
\hline C5A & $0.49274(15)$ & $0.23975(14)$ & $0.45181(11)$ & 0.0208 (3) \\
\hline C6A & $0.47284(15)$ & $0.23477(15)$ & $0.54949(12)$ & $0.0214(3)$ \\
\hline C7A & $0.72207(17)$ & $0.47425(16)$ & $0.69690(12)$ & $0.0266(3)$ \\
\hline H7AA & 0.6936 & 0.4592 & 0.7601 & $0.040 *$ \\
\hline $\mathrm{H} 7 \mathrm{AB}$ & 0.7056 & 0.5643 & 0.6754 & $0.040 *$ \\
\hline $\mathrm{H} 7 \mathrm{AC}$ & 0.8208 & 0.4547 & 0.7127 & $0.040^{*}$ \\
\hline C8A & $0.36724(17)$ & $0.14862(16)$ & $0.57215(13)$ & $0.0277(4)$ \\
\hline H8AA & 0.3599 & 0.1683 & 0.6425 & $0.042 *$ \\
\hline H8AB & 0.3955 & 0.0590 & 0.5701 & $0.042 *$ \\
\hline $\mathrm{H} 8 \mathrm{AC}$ & 0.2772 & 0.1622 & 0.5187 & $0.042^{*}$ \\
\hline O1B & $0.81428(11)$ & $0.52642(10)$ & $0.33828(8)$ & $0.0236(3)$ \\
\hline $\mathrm{O} 2 \mathrm{~B}$ & $0.84927(12)$ & $0.58025(11)$ & $0.50792(8)$ & $0.0261(3)$ \\
\hline $\mathrm{H} 2 \mathrm{~B}$ & $0.787(3)$ & $0.521(2)$ & $0.5057(18)$ & $0.040(6)^{*}$ \\
\hline C1B & 0.96195 (14) & $0.70251(14)$ & $0.40863(12)$ & $0.0207(3)$ \\
\hline $\mathrm{C} 2 \mathrm{~B}$ & $1.03036(16)$ & $0.77548(16)$ & $0.49703(12)$ & $0.0271(3)$ \\
\hline $\mathrm{H} 2 \mathrm{BA}$ & 1.0184 & 0.7568 & 0.5638 & $0.032 *$ \\
\hline C3B & $1.11636(19)$ & $0.87588(18)$ & $0.48757(14)$ & $0.0322(4)$ \\
\hline H3BA & 1.1636 & 0.9253 & 0.5480 & $0.039 *$ \\
\hline C4B & $1.13303(18)$ & $0.90380(17)$ & 0.38984 (14) & $0.0307(4)$ \\
\hline H4BA & 1.1919 & 0.9722 & 0.3835 & $0.037 *$ \\
\hline $\mathrm{C} 5 \mathrm{~B}$ & $1.06404(19)$ & $0.83225(18)$ & 0.30168 (14) & $0.0317(4)$ \\
\hline H5BA & 1.0750 & 0.8521 & 0.2348 & $0.038^{*}$ \\
\hline C6B & $0.97869(17)$ & $0.73133(16)$ & $0.31075(12)$ & $0.0272(3)$ \\
\hline H6BA & 0.9318 & 0.6820 & 0.2502 & $0.033^{*}$ \\
\hline C7B & $0.86845(14)$ & $0.59440(14)$ & $0.41483(11)$ & $0.0200(3)$ \\
\hline
\end{tabular}


Atomic displacement parameters $\left(\AA^{2}\right)$

\begin{tabular}{lllllll}
\hline & $U^{11}$ & $U^{22}$ & $U^{33}$ & $U^{12}$ & $U^{13}$ & $U^{23}$ \\
\hline C11 & $0.0262(2)$ & $0.0295(2)$ & $0.0204(2)$ & $-0.00904(13)$ & $0.00707(15)$ & $-0.00331(12)$ \\
N1A & $0.0225(6)$ & $0.0257(7)$ & $0.0168(6)$ & $-0.0013(5)$ & $0.0069(5)$ & $0.0004(5)$ \\
C2A & $0.0209(7)$ & $0.0233(7)$ & $0.0180(6)$ & $0.0017(6)$ & $0.0073(5)$ & $0.0011(6)$ \\
N3A & $0.0205(6)$ & $0.0230(6)$ & $0.0175(6)$ & $-0.0014(5)$ & $0.0069(5)$ & $-0.0008(5)$ \\
N4A & $0.0270(7)$ & $0.0298(7)$ & $0.0175(6)$ & $-0.0099(6)$ & $0.0097(5)$ & $-0.0038(5)$ \\
C4A & $0.0186(6)$ & $0.0226(7)$ & $0.0179(7)$ & $0.0007(5)$ & $0.0061(5)$ & $0.0003(5)$ \\
C5A & $0.0201(7)$ & $0.0233(7)$ & $0.0190(7)$ & $-0.0022(5)$ & $0.0058(5)$ & $0.0001(5)$ \\
C6A & $0.0199(7)$ & $0.0237(7)$ & $0.0214(7)$ & $0.0003(5)$ & $0.0073(5)$ & $0.0026(6)$ \\
C7A & $0.0300(8)$ & $0.0303(8)$ & $0.0196(7)$ & $-0.0042(6)$ & $0.0079(6)$ & $-0.0038(6)$ \\
C8A & $0.0271(8)$ & $0.0331(9)$ & $0.0255(8)$ & $-0.0078(6)$ & $0.0119(7)$ & $0.0017(6)$ \\
O1B & $0.0252(5)$ & $0.0249(6)$ & $0.0220(5)$ & $-0.0050(4)$ & $0.0092(4)$ & $-0.0015(4)$ \\
O2B & $0.0303(6)$ & $0.0296(6)$ & $0.0204(5)$ & $-0.0098(5)$ & $0.0109(4)$ & $-0.0009(4)$ \\
C1B & $0.0188(6)$ & $0.0213(7)$ & $0.0226(7)$ & $-0.0003(5)$ & $0.0073(5)$ & $0.0020(6)$ \\
C2B & $0.0296(8)$ & $0.0302(8)$ & $0.0210(7)$ & $-0.0069(6)$ & $0.0070(6)$ & $-0.0004(6)$ \\
C3B & $0.0346(9)$ & $0.0332(9)$ & $0.0270(8)$ & $-0.0126(7)$ & $0.0068(7)$ & $-0.0021(7)$ \\
C4B & $0.0312(8)$ & $0.0290(8)$ & $0.0330(8)$ & $-0.0094(7)$ & $0.0111(7)$ & $0.0014(7)$ \\
C5B & $0.0375(9)$ & $0.0347(9)$ & $0.0263(8)$ & $-0.0102(7)$ & $0.0148(7)$ & $0.0010(7)$ \\
C6B & $0.0306(8)$ & $0.0303(8)$ & $0.0228(7)$ & $-0.0073(7)$ & $0.0113(6)$ & $-0.0019(6)$ \\
C7B & $0.0180(6)$ & $0.0224(7)$ & $0.0203(7)$ & $0.0018(5)$ & $0.0070(5)$ & $0.0020(5)$ \\
& & & & & & \\
\hline
\end{tabular}

Geometric parameters $\left(A,{ }^{\circ}\right)$

\begin{tabular}{llll}
\hline C11-C5A & $1.7316(15)$ & C8A-H8AC & 0.9800 \\
N1A-C2A & $1.335(2)$ & O1B-C7B & $1.2224(18)$ \\
N1A-C6A & $1.357(2)$ & O2B-C7B & $1.3160(18)$ \\
C2A-N3A & $1.3411(18)$ & O2B-H2B & $0.87(3)$ \\
C2A-C7A & $1.500(2)$ & C1B-C2B & $1.392(2)$ \\
N3A-C4A & $1.3557(19)$ & C1B-C6B & $1.393(2)$ \\
N4A-C4A & $1.3320(19)$ & C1B-C7B & $1.491(2)$ \\
N4A-H41 & $0.86(2)$ & C2B-C3B & $1.392(2)$ \\
N4A-H42 & $0.92(2)$ & C2B-H2BA & 0.9500 \\
C4A-C5A & $1.415(2)$ & C3B-C4B & $1.389(2)$ \\
C5A-C6A & $1.372(2)$ & C3B-H3BA & 0.9500 \\
C6A-C8A & $1.497(2)$ & C4B-C5B & $1.383(2)$ \\
C7A-H7AA & 0.9800 & C4B-H4BA & 0.9500 \\
C7A-H7AB & 0.9800 & C5B-C6B & $1.391(2)$ \\
C7A-H7AC & 0.9800 & C5B-H5BA & 0.9500 \\
C8A-H8AA & 0.9800 & C6B-H6BA & 0.9500 \\
C8A-H8AB & 0.9800 & & 109.5 \\
C2A-N1A-C6A & $116.86(12)$ & C6A-C8A-H8AC & 109.5 \\
N1A-C2A-N3A & $126.03(14)$ & H8AA-C8A-H8AC & 109.5 \\
N1A-C2A-C7A & $117.24(12)$ & H8AB-C8A-H8AC & $111.1(15)$ \\
N3A-C2A-C7A & $116.73(13)$ & C7B-O2B-H2B & $119.75(14)$ \\
C2A-N3A-C4A & $118.18(12)$ & C2B-C1B-C6B
\end{tabular}




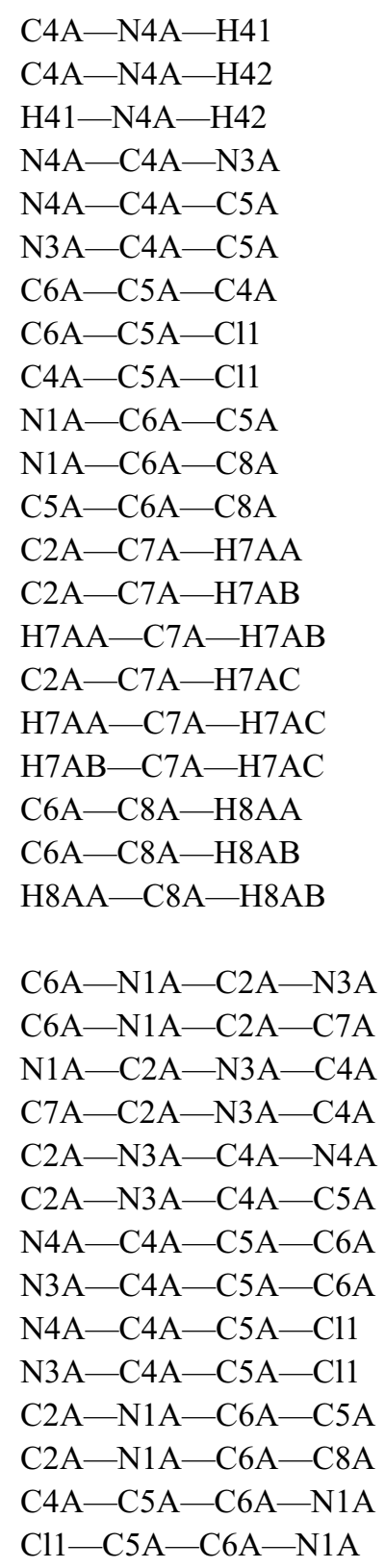

$115.3(14)$

$120.6(13)$

$122.8(19)$

$118.82(13)$

$122.81(14)$

$118.36(13)$

$119.74(13)$

$121.90(11)$

118.34 (11)

$120.74(13)$

$117.03(13)$

$122.23(14)$

109.5

109.5

109.5

109.5

109.5

109.5

109.5

109.5

109.5

$$
\begin{aligned}
& -1.5(2) \\
& 178.46(13) \\
& -1.2(2) \\
& 178.89(13) \\
& -178.15(14) \\
& 2.7(2) \\
& 179.16(14) \\
& -1.7(2) \\
& -2.3(2) \\
& 176.75(11) \\
& 2.5(2) \\
& -177.49(13) \\
& -0.9(2) \\
& -179.35(11)
\end{aligned}
$$

$$
\begin{aligned}
& \mathrm{C} 2 \mathrm{~B}-\mathrm{C} 1 \mathrm{~B}-\mathrm{C} 7 \mathrm{~B} \\
& \mathrm{C} 6 \mathrm{~B}-\mathrm{C} 1 \mathrm{~B}-\mathrm{C} 7 \mathrm{~B} \\
& \mathrm{C} 1 \mathrm{~B}-\mathrm{C} 2 \mathrm{~B}-\mathrm{C} 3 \mathrm{~B} \\
& \mathrm{C} 1 \mathrm{~B}-\mathrm{C} 2 \mathrm{~B}-\mathrm{H} 2 \mathrm{BA} \\
& \mathrm{C} 3 \mathrm{~B}-\mathrm{C} 2 \mathrm{~B}-\mathrm{H} 2 \mathrm{BA} \\
& \mathrm{C} 4 \mathrm{~B}-\mathrm{C} 3 \mathrm{~B}-\mathrm{C} 2 \mathrm{~B} \\
& \mathrm{C} 4 \mathrm{~B}-\mathrm{C} 3 \mathrm{~B}-\mathrm{H} 3 \mathrm{BA} \\
& \mathrm{C} 2 \mathrm{~B}-\mathrm{C} 3 \mathrm{~B}-\mathrm{H} 3 \mathrm{BA} \\
& \mathrm{C} 5 \mathrm{~B}-\mathrm{C} 4 \mathrm{~B}-\mathrm{C} 3 \mathrm{~B} \\
& \mathrm{C} 5 \mathrm{~B}-\mathrm{C} 4 \mathrm{~B}-\mathrm{H} 4 \mathrm{BA} \\
& \mathrm{C} 3 \mathrm{~B}-\mathrm{C} 4 \mathrm{~B}-\mathrm{H} 4 \mathrm{BA} \\
& \mathrm{C} 4 \mathrm{~B}-\mathrm{C} 5 \mathrm{~B}-\mathrm{C} 6 \mathrm{~B} \\
& \mathrm{C} 4 \mathrm{~B}-\mathrm{C} 5 \mathrm{~B}-\mathrm{H} 5 \mathrm{BA} \\
& \mathrm{C} 6 \mathrm{~B}-\mathrm{C} 5 \mathrm{~B}-\mathrm{H} 5 \mathrm{BA} \\
& \mathrm{C} 5 \mathrm{~B}-\mathrm{C} 6 \mathrm{~B}-\mathrm{C} 1 \mathrm{~B} \\
& \mathrm{C} 5 \mathrm{~B}-\mathrm{C} 6 \mathrm{~B}-\mathrm{H} 6 \mathrm{BA} \\
& \mathrm{C} 1 \mathrm{~B}-\mathrm{C} 6 \mathrm{~B}-\mathrm{H} 6 \mathrm{BA} \\
& \mathrm{O} 1 \mathrm{~B}-\mathrm{C} 7 \mathrm{~B}-\mathrm{O} 2 \mathrm{~B} \\
& \text { O1B-C7B-C1B } \\
& \text { O2B-C7B-C1B }
\end{aligned}
$$

$121.87(13)$

$118.37(13)$

$119.95(15)$

120.0

120.0

$120.00(16)$

120.0

120.0

120.14 (15)

119.9

119.9

$120.12(15)$

119.9

119.9

120.02 (15)

120.0

120.0

123.85 (13)

$121.60(13)$

114.54 (13)

$179.03(14)$

$0.6(2)$

-0.7 (2)

$-179.78(15)$

0.5 (3)

$0.2(3)$

$-0.6(3)$

$0.3(3)$

0.3 (2)

$179.40(15)$

-176.15 (14)

4.8 (2)

4.4 (2)

$-174.61(13)$

Hydrogen-bond geometry $\left(\AA,{ }^{\circ}\right)$

\begin{tabular}{lllll}
\hline$D-\mathrm{H} \cdots A$ & $D-\mathrm{H}$ & $\mathrm{H} \cdots A$ & $D \cdots A$ & $D-\mathrm{H} \cdots A$ \\
\hline $\mathrm{N} 4 A-\mathrm{H} 41 \cdots \mathrm{O} 1 B$ & $0.86(2)$ & $1.99(2)$ & $2.8419(17)$ & $173(2)$ \\
$\mathrm{N} 4 A-\mathrm{H} 42 \cdots \mathrm{N} 1 A^{\mathrm{i}}$ & $0.92(2)$ & $2.21(2)$ & $3.0617(18)$ & $153.5(18)$ \\
$\mathrm{C} 8 A-\mathrm{H} 8 A B \cdots \mathrm{C} 11^{\mathrm{ii}}$ & 0.98 & 2.98 & $3.8571(18)$ & 149 \\
$\mathrm{O} 2 B-\mathrm{H} 2 B \cdots \mathrm{N} 3 A$ & $0.87(3)$ & $1.80(3)$ & $2.6712(17)$ & $174(2)$ \\
\hline
\end{tabular}

Symmetry codes: (i) $x,-y+1 / 2, z-1 / 2$; (ii) $-x+1,-y,-z+1$. 
4-Amino-5-chloro-2,6-dimethylpyrimidine-2-methylbenzoic acid (1/1) (COCRYSTAL-7)

Crystal data

$\mathrm{C}_{6} \mathrm{H}_{8} \mathrm{ClN}_{3} \cdot \mathrm{C}_{8} \mathrm{H}_{8} \mathrm{O}_{2}$

$M_{r}=293.75$

Orthorhombic, $P 2{ }_{1} 2_{1} 2_{1}$

$a=7.3992(2) \AA$

$b=13.9842(4) \AA$

$c=26.9897(8) \AA$

$V=2792.68(14) \AA^{3}$

$Z=8$

$F(000)=1232$

Data collection

Agilent SuperNova Dual Source diffractometer with an Atlas detector

Radiation source: sealed X-ray tube Detector resolution: 10.6501 pixels $\mathrm{mm}^{-1}$ $\omega$ scans

Absorption correction: multi-scan

(CrysAlis PRO; Agilent, 2014)

$T_{\min }=0.740, T_{\max }=1.000$

\section{Refinement}

Refinement on $F^{2}$

Least-squares matrix: full

$R\left[F^{2}>2 \sigma\left(F^{2}\right)\right]=0.034$

$w R\left(F^{2}\right)=0.091$

$S=1.04$

4966 reflections

376 parameters

0 restraints

Hydrogen site location: mixed

$\mathrm{H}$ atoms treated by a mixture of independent and constrained refinement
$D_{\mathrm{x}}=1.397 \mathrm{Mg} \mathrm{m}^{-3}$

$\mathrm{Cu} K \alpha$ radiation, $\lambda=1.54184 \AA$

Cell parameters from 3914 reflections

$\theta=3.6-76.5^{\circ}$

$\mu=2.47 \mathrm{~mm}^{-1}$

$T=120 \mathrm{~K}$

Needle, colorless

$0.49 \times 0.11 \times 0.08 \mathrm{~mm}$

8897 measured reflections

4966 independent reflections

4617 reflections with $I>2 \sigma(I)$

$R_{\text {int }}=0.030$

$\theta_{\text {max }}=76.8^{\circ}, \theta_{\min }=3.3^{\circ}$

$h=-7 \rightarrow 9$

$k=-17 \rightarrow 14$

$l=-22 \rightarrow 34$

$w=1 /\left[\sigma^{2}\left(F_{\mathrm{o}}^{2}\right)+(0.0488 P)^{2}+0.3891 P\right]$ where $P=\left(F_{\mathrm{o}}^{2}+2 F_{\mathrm{c}}^{2}\right) / 3$

$(\Delta / \sigma)_{\max }=0.002$

$\Delta \rho_{\max }=0.24$ e $\AA^{-3}$

$\Delta \rho_{\min }=-0.24$ e $\AA^{-3}$

Extinction correction: SHELXL2014

(Sheldrick, 2015b), $\mathrm{Fc}^{*}=\mathrm{kFc}\left[1+0.001 \mathrm{xFc}^{2} \lambda^{3} / \sin (2 \theta)\right]^{-1 / 4}$

Extinction coefficient: 0.00039 (10)

Absolute structure: Flack $x$ determined using 1451 quotients $[(\mathrm{I}+)-(\mathrm{I}-)] /[(\mathrm{I}+)+(\mathrm{I}-)]$ (Parsons et al., 2013)

Absolute structure parameter: -0.001 (10)

Special details

Geometry. All esds (except the esd in the dihedral angle between two 1.s. planes) are estimated using the full covariance matrix. The cell esds are taken into account individually in the estimation of esds in distances, angles and torsion angles; correlations between esds in cell parameters are only used when they are defined by crystal symmetry. An approximate (isotropic) treatment of cell esds is used for estimating esds involving l.s. planes.

Fractional atomic coordinates and isotropic or equivalent isotropic displacement parameters $\left(\AA^{2}\right)$

\begin{tabular}{lllll}
\hline & $x$ & $y$ & $z$ & $U_{\text {iso }} * / U_{\text {eq }}$ \\
\hline Cl1 & $0.91235(8)$ & $0.87398(4)$ & $0.43040(2)$ & $0.02206(15)$ \\
N1A & $0.7729(3)$ & $1.10678(15)$ & $0.50704(8)$ & $0.0201(4)$ \\
C2A & $0.7196(4)$ & $1.06463(18)$ & $0.54874(9)$ & $0.0192(5)$ \\
N3A & $0.7172(3)$ & $0.97006(15)$ & $0.55756(8)$ & $0.0191(4)$ \\
N4A & $0.7653(4)$ & $0.81632(16)$ & $0.53005(8)$ & $0.0234(5)$ \\
H4AA & 0.7255 & 0.7950 & 0.5587 & $0.028^{*}$
\end{tabular}




\begin{tabular}{|c|c|c|c|c|}
\hline H4AB & 0.8004 & 0.7757 & 0.5071 & $0.028^{*}$ \\
\hline $\mathrm{C} 4 \mathrm{~A}$ & $0.7723(4)$ & $0.91023(18)$ & $0.52138(9)$ & $0.0183(5)$ \\
\hline C5A & $0.8346(4)$ & 0.95017 (19) & $0.47634(9)$ & $0.0185(5)$ \\
\hline C6A & $0.8313(4)$ & $1.0482(2)$ & $0.47023(10)$ & $0.0204(5)$ \\
\hline C7A & $0.6540(4)$ & 1.12797 (19) & $0.58987(9)$ & $0.0231(5)$ \\
\hline H7AA & 0.7146 & 1.1902 & 0.5878 & $0.035^{*}$ \\
\hline H7AB & 0.6814 & 1.0982 & 0.6219 & $0.035^{*}$ \\
\hline H7AC & 0.5231 & 1.1370 & 0.5868 & $0.035^{*}$ \\
\hline C8A & $0.8890(4)$ & $1.0961(2)$ & $0.42303(10)$ & $0.0258(6)$ \\
\hline H8AA & 0.9284 & 1.1616 & 0.4302 & $0.039 *$ \\
\hline H8AB & 0.7871 & 1.0978 & 0.3999 & $0.039 *$ \\
\hline $\mathrm{H} 8 \mathrm{AC}$ & 0.9892 & 1.0603 & 0.4082 & $0.039 *$ \\
\hline $\mathrm{Cl} 2$ & $0.88558(8)$ & $0.36846(4)$ & $0.56903(2)$ & $0.02260(15)$ \\
\hline N1B & $0.7984(3)$ & $0.60749(15)$ & $0.49210(8)$ & $0.0203(4)$ \\
\hline $\mathrm{C} 2 \mathrm{~B}$ & 0.7345 (4) & $0.56860(18)$ & $0.45060(9)$ & $0.0186(5)$ \\
\hline N3B & $0.7075(3)$ & $0.47481(15)$ & $0.44219(8)$ & $0.0190(4)$ \\
\hline N4B & $0.7245(4)$ & $0.31988(16)$ & $0.46998(8)$ & $0.0231(5)$ \\
\hline H4BA & 0.6796 & 0.3010 & 0.4414 & $0.028^{*}$ \\
\hline H4BB & 0.7517 & 0.2775 & 0.4929 & $0.028^{*}$ \\
\hline C4B & $0.7514(4)$ & $0.41261(18)$ & $0.47854(9)$ & $0.0190(5)$ \\
\hline C5B & $0.8238(4)$ & 0.44877 (19) & $0.52313(9)$ & $0.0186(5)$ \\
\hline C6B & $0.8434(3)$ & $0.54633(19)$ & $0.52929(9)$ & $0.0190(5)$ \\
\hline C7B & $0.6863(4)$ & 0.63491 (19) & $0.40878(10)$ & $0.0250(5)$ \\
\hline H7BA & 0.6899 & 0.7012 & 0.4205 & $0.037^{*}$ \\
\hline H7BB & 0.5645 & 0.6200 & 0.3968 & $0.037^{*}$ \\
\hline $\mathrm{H} 7 \mathrm{BC}$ & 0.7732 & 0.6268 & 0.3817 & $0.037^{*}$ \\
\hline C8B & $0.9118(4)$ & 0.59057 (19) & $0.57601(10)$ & $0.0238(5)$ \\
\hline H8BA & 0.9847 & 0.6470 & 0.5680 & $0.036^{*}$ \\
\hline H8BB & 0.9864 & 0.5441 & 0.5939 & $0.036^{*}$ \\
\hline H8BC & 0.8094 & 0.6096 & 0.5968 & $0.036^{*}$ \\
\hline $\mathrm{O} 1 \mathrm{C}$ & $0.6542(4)$ & $0.92635(14)$ & $0.65200(7)$ & $0.0346(6)$ \\
\hline $\mathrm{H} 1 \mathrm{C}$ & $0.675(7)$ & $0.938(4)$ & $0.6173(19)$ & $0.071(16)^{*}$ \\
\hline $\mathrm{O} 2 \mathrm{C}$ & $0.6384(3)$ & $0.77291(14)$ & $0.63071(7)$ & $0.0288(5)$ \\
\hline $\mathrm{C} 1 \mathrm{C}$ & $0.5787(4)$ & $0.81731(18)$ & $0.71489(9)$ & $0.0191(5)$ \\
\hline $\mathrm{C} 2 \mathrm{C}$ & $0.5895(4)$ & $0.72517(18)$ & $0.73568(9)$ & $0.0195(5)$ \\
\hline $\mathrm{C} 3 \mathrm{C}$ & $0.5330(4)$ & $0.71465(18)$ & $0.78504(9)$ & $0.0217(5)$ \\
\hline $\mathrm{H} 3 \mathrm{CA}$ & 0.5403 & 0.6535 & 0.8002 & $0.026^{*}$ \\
\hline $\mathrm{C} 4 \mathrm{C}$ & $0.4672(4)$ & $0.7903(2)$ & $0.81212(9)$ & $0.0245(5)$ \\
\hline $\mathrm{H} 4 \mathrm{CA}$ & 0.4281 & 0.7805 & 0.8453 & $0.029^{*}$ \\
\hline C5C & $0.4574(4)$ & 0.88148 (19) & $0.79126(10)$ & $0.0239(5)$ \\
\hline $\mathrm{H} 5 \mathrm{CA}$ & 0.4119 & 0.9339 & 0.8098 & $0.029^{*}$ \\
\hline C6C & $0.5155(4)$ & $0.89400(17)$ & $0.74291(9)$ & $0.0213(5)$ \\
\hline H6CA & 0.5121 & 0.9560 & 0.7286 & $0.026^{*}$ \\
\hline C7C & $0.6283(4)$ & $0.83490(18)$ & $0.66198(10)$ & $0.0205(5)$ \\
\hline $\mathrm{C} 8 \mathrm{C}$ & $0.6594(4)$ & $0.63891(18)$ & $0.70853(10)$ & $0.0228(5)$ \\
\hline H8CA & 0.7754 & 0.6544 & 0.6930 & $0.034^{*}$ \\
\hline H8CB & 0.5723 & 0.6200 & 0.6830 & $0.034^{*}$ \\
\hline H8CC & 0.6759 & 0.5861 & 0.7320 & $0.034^{*}$ \\
\hline
\end{tabular}




$\begin{array}{lllll}\text { O1D } & 0.5864(3) & 0.43696(13) & 0.35050(7) & 0.0299(5) \\ \text { H1D } & 0.633(6) & 0.443(3) & 0.3830(16) & 0.049(12)^{*} \\ \text { O2D } & 0.5744(3) & 0.28307(13) & 0.37102(7) & 0.0258(4) \\ \text { C1D } & 0.4706(4) & 0.33139(18) & 0.29067(9) & 0.0187(5) \\ \text { C2D } & 0.4639(4) & 0.23980(18) & 0.26889(9) & 0.0206(5) \\ \text { C3D } & 0.3967(4) & 0.23334(19) & 0.22030(10) & 0.0224(5) \\ \text { H3DA } & 0.3961 & 0.1729 & 0.2042 & 0.027^{*} \\ \text { C4D } & 0.3317(4) & 0.3120(2) & 0.19525(10) & 0.0246(5) \\ \text { H4DA } & 0.2858 & 0.3050 & 0.1626 & 0.029^{*} \\ \text { C5D } & 0.3330(4) & 0.40158(19) & 0.21763(10) & 0.0242(5) \\ \text { H5DA } & 0.2869 & 0.4558 & 0.2006 & 0.029^{*} \\ \text { C6D } & 0.4023(4) & 0.41093(18) & 0.26514(10) & 0.0209(5) \\ \text { H6DA } & 0.4036 & 0.4720 & 0.2806 & 0.025^{*} \\ \text { C7D } & 0.5482(4) & 0.34623(18) & 0.34092(9) & 0.0198(5) \\ \text { C8D } & 0.5261(4) & 0.14993(18) & 0.29421(10) & 0.0250(6) \\ \text { H8DA } & 0.5127 & 0.0956 & 0.2715 & 0.037^{*} \\ \text { H8DB } & 0.4529 & 0.1390 & 0.3239 & 0.037^{*} \\ \text { H8DC } & 0.6534 & 0.1566 & 0.3037 & 0.037^{*} \\ & & & & \end{array}$

Atomic displacement parameters $\left(\AA^{2}\right)$

\begin{tabular}{lllllll}
\hline & $U^{11}$ & $U^{22}$ & $U^{33}$ & $U^{12}$ & $U^{13}$ & $U^{23}$ \\
\hline $\mathrm{C} 11$ & $0.0255(3)$ & $0.0205(3)$ & $0.0201(3)$ & $0.0010(2)$ & $0.0027(2)$ & $-0.0043(2)$ \\
$\mathrm{N} 1 \mathrm{~A}$ & $0.0225(11)$ & $0.0162(10)$ & $0.0217(9)$ & $-0.0022(9)$ & $0.0007(9)$ & $0.0010(8)$ \\
$\mathrm{C} 2 \mathrm{~A}$ & $0.0203(12)$ & $0.0162(12)$ & $0.0211(11)$ & $-0.0015(10)$ & $-0.0003(10)$ & $-0.0003(9)$ \\
$\mathrm{N} 3 \mathrm{~A}$ & $0.0231(10)$ & $0.0153(10)$ & $0.0190(9)$ & $-0.0012(9)$ & $0.0020(8)$ & $-0.0005(8)$ \\
$\mathrm{N} 4 \mathrm{~A}$ & $0.0345(13)$ & $0.0151(10)$ & $0.0207(9)$ & $-0.0004(10)$ & $0.0053(10)$ & $-0.0011(8)$ \\
$\mathrm{C} 4 \mathrm{~A}$ & $0.0196(12)$ & $0.0148(11)$ & $0.0206(11)$ & $0.0015(10)$ & $-0.0021(10)$ & $-0.0010(9)$ \\
$\mathrm{C} 5 \mathrm{~A}$ & $0.0200(12)$ & $0.0188(12)$ & $0.0168(10)$ & $0.0004(10)$ & $0.0008(10)$ & $-0.0023(10)$ \\
$\mathrm{C} 6 \mathrm{~A}$ & $0.0197(12)$ & $0.0209(12)$ & $0.0204(11)$ & $0.0014(11)$ & $0.0004(10)$ & $0.0002(10)$ \\
$\mathrm{C} 7 \mathrm{~A}$ & $0.0301(13)$ & $0.0165(11)$ & $0.0227(11)$ & $0.0000(11)$ & $0.0038(11)$ & $-0.0013(10)$ \\
$\mathrm{C} 8 \mathrm{~A}$ & $0.0303(13)$ & $0.0241(12)$ & $0.0231(12)$ & $0.0008(12)$ & $0.0052(12)$ & $0.0042(11)$ \\
$\mathrm{C} 12$ & $0.0268(3)$ & $0.0200(3)$ & $0.0210(3)$ & $0.0018(2)$ & $-0.0034(2)$ & $0.0040(2)$ \\
$\mathrm{N} 1 \mathrm{~B}$ & $0.0237(11)$ & $0.0161(9)$ & $0.0212(9)$ & $0.0016(9)$ & $-0.0007(9)$ & $-0.0019(8)$ \\
$\mathrm{C} 2 \mathrm{~B}$ & $0.0215(12)$ & $0.0141(11)$ & $0.0202(11)$ & $0.0021(10)$ & $0.0012(10)$ & $-0.0005(9)$ \\
$\mathrm{N} 3 \mathrm{~B}$ & $0.0242(10)$ & $0.0153(10)$ & $0.0175(9)$ & $0.0003(9)$ & $0.0006(8)$ & $-0.0007(8)$ \\
N4B & $0.0333(12)$ & $0.0149(9)$ & $0.0211(9)$ & $-0.0012(10)$ & $-0.0050(10)$ & $0.0009(8)$ \\
$\mathrm{C} 4 \mathrm{~B}$ & $0.0205(12)$ & $0.0161(11)$ & $0.0204(11)$ & $0.0008(10)$ & $0.0035(10)$ & $0.0000(9)$ \\
$\mathrm{C} 5 \mathrm{~B}$ & $0.0204(12)$ & $0.0165(11)$ & $0.0188(11)$ & $0.0041(10)$ & $0.0001(10)$ & $0.0006(10)$ \\
$\mathrm{C} 6 \mathrm{~B}$ & $0.0178(12)$ & $0.0194(12)$ & $0.0199(11)$ & $0.0023(10)$ & $0.0002(10)$ & $-0.0015(10)$ \\
$\mathrm{C} 7 \mathrm{~B}$ & $0.0348(14)$ & $0.0166(11)$ & $0.0235(11)$ & $-0.0006(12)$ & $-0.0045(11)$ & $0.0028(10)$ \\
$\mathrm{C} 8 \mathrm{~B}$ & $0.0255(13)$ & $0.0215(12)$ & $0.0244(12)$ & $0.0018(11)$ & $-0.0060(11)$ & $-0.0050(10)$ \\
O1C & $0.0660(17)$ & $0.0175(9)$ & $0.0202(9)$ & $-0.0079(10)$ & $0.0104(10)$ & $-0.0017(7)$ \\
O2C & $0.0468(13)$ & $0.0190(9)$ & $0.0206(8)$ & $-0.0022(9)$ & $0.0030(9)$ & $-0.0005(7)$ \\
C1C & $0.0196(12)$ & $0.0166(11)$ & $0.0211(11)$ & $-0.0019(10)$ & $-0.0006(10)$ & $-0.0001(9)$ \\
C2C & $0.0200(12)$ & $0.0173(11)$ & $0.0211(11)$ & $-0.0024(11)$ & $-0.0010(10)$ & $0.0004(9)$ \\
C3C & $0.0257(13)$ & $0.0173(11)$ & $0.0221(11)$ & $-0.0039(11)$ & $-0.0018(11)$ & $0.0037(9)$ \\
C4C & $0.0281(13)$ & $0.0269(13)$ & $0.0186(11)$ & $-0.0054(12)$ & $0.0015(11)$ & $0.0003(10)$ \\
& & & & &
\end{tabular}




\begin{tabular}{lllllll} 
& & & & & \\
C5C & $0.0284(13)$ & $0.0211(12)$ & $0.0222(11)$ & $-0.0011(11)$ & $0.0030(11)$ & $-0.0029(10)$ \\
C6C & $0.0257(13)$ & $0.0156(10)$ & $0.0227(11)$ & $-0.0017(10)$ & $-0.0008(11)$ & $0.0019(10)$ \\
C7C & $0.0227(12)$ & $0.0168(11)$ & $0.0218(11)$ & $-0.0034(10)$ & $-0.0005(10)$ & $0.0014(9)$ \\
C8C & $0.0276(13)$ & $0.0149(11)$ & $0.0260(12)$ & $-0.0016(11)$ & $0.0011(11)$ & $0.0013(10)$ \\
O1D & $0.0491(13)$ & $0.0156(8)$ & $0.0250(9)$ & $-0.0055(9)$ & $-0.0112(10)$ & $-0.0004(7)$ \\
O2D & $0.0385(11)$ & $0.0180(8)$ & $0.0208(8)$ & $-0.0034(9)$ & $-0.0039(9)$ & $0.0012(7)$ \\
C1D & $0.0198(12)$ & $0.0175(11)$ & $0.0186(10)$ & $-0.0012(10)$ & $0.0005(10)$ & $-0.0001(9)$ \\
C2D & $0.0218(13)$ & $0.0184(11)$ & $0.0215(11)$ & $-0.0002(10)$ & $0.0015(10)$ & $-0.0004(9)$ \\
C3D & $0.0236(13)$ & $0.0213(12)$ & $0.0222(12)$ & $-0.0030(11)$ & $0.0010(10)$ & $-0.0057(10)$ \\
C4D & $0.0235(13)$ & $0.0287(13)$ & $0.0215(11)$ & $-0.0035(11)$ & $-0.0031(11)$ & $0.0001(10)$ \\
C5D & $0.0249(13)$ & $0.0210(12)$ & $0.0266(12)$ & $-0.0006(11)$ & $-0.0034(11)$ & $0.0046(10)$ \\
C6D & $0.0228(12)$ & $0.0153(10)$ & $0.0246(12)$ & $-0.0016(11)$ & $0.0013(11)$ & $-0.0010(9)$ \\
C7D & $0.0219(11)$ & $0.0173(11)$ & $0.0203(11)$ & $-0.0019(10)$ & $0.0021(10)$ & $-0.0026(9)$ \\
C8D & $0.0320(14)$ & $0.0170(11)$ & $0.0258(12)$ & $0.0019(11)$ & $-0.0012(11)$ & $-0.0024(10)$ \\
& & & & & & \\
\hline
\end{tabular}

Geometric parameters $\left(A,{ }^{\circ}\right)$

\begin{tabular}{|c|c|c|c|}
\hline $\mathrm{Cl} 1-\mathrm{C} 5 \mathrm{~A}$ & $1.733(3)$ & $\mathrm{O} 1 \mathrm{C}-\mathrm{C} 7 \mathrm{C}$ & $1.321(3)$ \\
\hline $\mathrm{N} 1 \mathrm{~A}-\mathrm{C} 2 \mathrm{~A}$ & $1.330(3)$ & $\mathrm{O} 1 \mathrm{C}-\mathrm{H} 1 \mathrm{C}$ & $0.96(5)$ \\
\hline $\mathrm{N} 1 \mathrm{~A}-\mathrm{C} 6 \mathrm{~A}$ & $1.359(3)$ & $\mathrm{O} 2 \mathrm{C}-\mathrm{C} 7 \mathrm{C}$ & $1.212(3)$ \\
\hline $\mathrm{C} 2 \mathrm{~A}-\mathrm{N} 3 \mathrm{~A}$ & $1.344(3)$ & $\mathrm{C} 1 \mathrm{C}-\mathrm{C} 6 \mathrm{C}$ & $1.393(4)$ \\
\hline $\mathrm{C} 2 \mathrm{~A}-\mathrm{C} 7 \mathrm{~A}$ & $1.501(4)$ & $\mathrm{C} 1 \mathrm{C}-\mathrm{C} 2 \mathrm{C}$ & $1.408(3)$ \\
\hline $\mathrm{N} 3 \mathrm{~A}-\mathrm{C} 4 \mathrm{~A}$ & $1.349(3)$ & $\mathrm{C} 1 \mathrm{C}-\mathrm{C} 7 \mathrm{C}$ & $1.495(3)$ \\
\hline $\mathrm{N} 4 \mathrm{~A}-\mathrm{C} 4 \mathrm{~A}$ & $1.335(3)$ & $\mathrm{C} 2 \mathrm{C}-\mathrm{C} 3 \mathrm{C}$ & $1.404(3)$ \\
\hline $\mathrm{N} 4 \mathrm{~A}-\mathrm{H} 4 \mathrm{AA}$ & 0.8800 & $\mathrm{C} 2 \mathrm{C}-\mathrm{C} 8 \mathrm{C}$ & $1.503(4)$ \\
\hline $\mathrm{N} 4 \mathrm{~A}-\mathrm{H} 4 \mathrm{AB}$ & 0.8800 & $\mathrm{C} 3 \mathrm{C}-\mathrm{C} 4 \mathrm{C}$ & $1.375(4)$ \\
\hline $\mathrm{C} 4 \mathrm{~A}-\mathrm{C} 5 \mathrm{~A}$ & $1.415(4)$ & $\mathrm{C} 3 \mathrm{C}-\mathrm{H} 3 \mathrm{CA}$ & 0.9500 \\
\hline $\mathrm{C} 5 \mathrm{~A}-\mathrm{C} 6 \mathrm{~A}$ & $1.380(4)$ & $\mathrm{C} 4 \mathrm{C}-\mathrm{C} 5 \mathrm{C}$ & $1.395(4)$ \\
\hline $\mathrm{C} 6 \mathrm{~A}-\mathrm{C} 8 \mathrm{~A}$ & $1.502(4)$ & $\mathrm{C} 4 \mathrm{C}-\mathrm{H} 4 \mathrm{CA}$ & 0.9500 \\
\hline C7A-H7AA & 0.9800 & $\mathrm{C} 5 \mathrm{C}-\mathrm{C} 6 \mathrm{C}$ & $1.385(4)$ \\
\hline $\mathrm{C} 7 \mathrm{~A}-\mathrm{H} 7 \mathrm{AB}$ & 0.9800 & $\mathrm{C} 5 \mathrm{C}-\mathrm{H} 5 \mathrm{CA}$ & 0.9500 \\
\hline $\mathrm{C} 7 \mathrm{~A}-\mathrm{H} 7 \mathrm{AC}$ & 0.9800 & $\mathrm{C} 6 \mathrm{C}-\mathrm{H} 6 \mathrm{CA}$ & 0.9500 \\
\hline C8A-H8AA & 0.9800 & $\mathrm{C} 8 \mathrm{C}-\mathrm{H} 8 \mathrm{CA}$ & 0.9800 \\
\hline $\mathrm{C} 8 \mathrm{~A}-\mathrm{H} 8 \mathrm{AB}$ & 0.9800 & $\mathrm{C} 8 \mathrm{C}-\mathrm{H} 8 \mathrm{CB}$ & 0.9800 \\
\hline $\mathrm{C} 8 \mathrm{~A}-\mathrm{H} 8 \mathrm{AC}$ & 0.9800 & $\mathrm{C} 8 \mathrm{C}-\mathrm{H} 8 \mathrm{CC}$ & 0.9800 \\
\hline $\mathrm{Cl} 2-\mathrm{C} 5 \mathrm{~B}$ & $1.734(3)$ & O1D-C7D & $1.325(3)$ \\
\hline $\mathrm{N} 1 \mathrm{~B}-\mathrm{C} 2 \mathrm{~B}$ & $1.332(3)$ & $\mathrm{O} 1 \mathrm{D}-\mathrm{H} 1 \mathrm{D}$ & $0.95(4)$ \\
\hline $\mathrm{N} 1 \mathrm{~B}-\mathrm{C} 6 \mathrm{~B}$ & $1.360(3)$ & $\mathrm{O} 2 \mathrm{D}-\mathrm{C} 7 \mathrm{D}$ & $1.216(3)$ \\
\hline $\mathrm{C} 2 \mathrm{~B}-\mathrm{N} 3 \mathrm{~B}$ & $1.346(3)$ & $\mathrm{C} 1 \mathrm{D}-\mathrm{C} 6 \mathrm{D}$ & $1.402(4)$ \\
\hline $\mathrm{C} 2 \mathrm{~B}-\mathrm{C} 7 \mathrm{~B}$ & $1.504(3)$ & $\mathrm{C} 1 \mathrm{D}-\mathrm{C} 2 \mathrm{D}$ & $1.410(4)$ \\
\hline $\mathrm{N} 3 \mathrm{~B}-\mathrm{C} 4 \mathrm{~B}$ & $1.351(3)$ & $\mathrm{C} 1 \mathrm{D}-\mathrm{C} 7 \mathrm{D}$ & $1.487(3)$ \\
\hline $\mathrm{N} 4 \mathrm{~B}-\mathrm{C} 4 \mathrm{~B}$ & $1.332(3)$ & $\mathrm{C} 2 \mathrm{D}-\mathrm{C} 3 \mathrm{D}$ & $1.406(4)$ \\
\hline $\mathrm{N} 4 \mathrm{~B}-\mathrm{H} 4 \mathrm{BA}$ & 0.8800 & $\mathrm{C} 2 \mathrm{D}-\mathrm{C} 8 \mathrm{D}$ & $1.503(4)$ \\
\hline $\mathrm{N} 4 \mathrm{~B}-\mathrm{H} 4 \mathrm{BB}$ & 0.8800 & $\mathrm{C} 3 \mathrm{D}-\mathrm{C} 4 \mathrm{D}$ & $1.378(4)$ \\
\hline $\mathrm{C} 4 \mathrm{~B}-\mathrm{C} 5 \mathrm{~B}$ & $1.411(4)$ & $\mathrm{C} 3 \mathrm{D}-\mathrm{H} 3 \mathrm{DA}$ & 0.9500 \\
\hline $\mathrm{C} 5 \mathrm{~B}-\mathrm{C} 6 \mathrm{~B}$ & $1.382(4)$ & $\mathrm{C} 4 \mathrm{D}-\mathrm{C} 5 \mathrm{D}$ & $1.391(4)$ \\
\hline $\mathrm{C} 6 \mathrm{~B}-\mathrm{C} 8 \mathrm{~B}$ & $1.493(3)$ & $\mathrm{C} 4 \mathrm{D}-\mathrm{H} 4 \mathrm{DA}$ & 0.9500 \\
\hline $\mathrm{C} 7 \mathrm{~B}-\mathrm{H} 7 \mathrm{BA}$ & 0.9800 & C5D—C6D & $1.387(4)$ \\
\hline
\end{tabular}




\begin{tabular}{|c|c|c|c|}
\hline $\mathrm{C} 7 \mathrm{~B}-\mathrm{H} 7 \mathrm{BB}$ & 0.9800 & C5D-H5DA & 0.9500 \\
\hline $\mathrm{C} 7 \mathrm{~B}-\mathrm{H} 7 \mathrm{BC}$ & 0.9800 & C6D-H6DA & 0.9500 \\
\hline $\mathrm{C} 8 \mathrm{~B}-\mathrm{H} 8 \mathrm{BA}$ & 0.9800 & $\mathrm{C} 8 \mathrm{D}-\mathrm{H} 8 \mathrm{DA}$ & 0.9800 \\
\hline $\mathrm{C} 8 \mathrm{~B}-\mathrm{H} 8 \mathrm{BB}$ & 0.9800 & $\mathrm{C} 8 \mathrm{D}-\mathrm{H} 8 \mathrm{DB}$ & 0.9800 \\
\hline $\mathrm{C} 8 \mathrm{~B}-\mathrm{H} 8 \mathrm{BC}$ & 0.9800 & $\mathrm{C} 8 \mathrm{D}-\mathrm{H} 8 \mathrm{DC}$ & 0.9800 \\
\hline $\mathrm{C} 2 \mathrm{~A}-\mathrm{N} 1 \mathrm{~A}-\mathrm{C} 6 \mathrm{~A}$ & $116.5(2)$ & $\mathrm{H} 8 \mathrm{BB}-\mathrm{C} 8 \mathrm{~B}-\mathrm{H} 8 \mathrm{BC}$ & 109.5 \\
\hline $\mathrm{N} 1 \mathrm{~A}-\mathrm{C} 2 \mathrm{~A}-\mathrm{N} 3 \mathrm{~A}$ & $126.1(2)$ & $\mathrm{C} 7 \mathrm{C}-\mathrm{O} 1 \mathrm{C}-\mathrm{H} 1 \mathrm{C}$ & $112(3)$ \\
\hline $\mathrm{N} 1 \mathrm{~A}-\mathrm{C} 2 \mathrm{~A}-\mathrm{C} 7 \mathrm{~A}$ & $117.4(2)$ & $\mathrm{C} 6 \mathrm{C}-\mathrm{C} 1 \mathrm{C}-\mathrm{C} 2 \mathrm{C}$ & $120.5(2)$ \\
\hline $\mathrm{N} 3 \mathrm{~A}-\mathrm{C} 2 \mathrm{~A}-\mathrm{C} 7 \mathrm{~A}$ & $116.5(2)$ & $\mathrm{C} 6 \mathrm{C}-\mathrm{C} 1 \mathrm{C}-\mathrm{C} 7 \mathrm{C}$ & $118.3(2)$ \\
\hline $\mathrm{C} 2 \mathrm{~A}-\mathrm{N} 3 \mathrm{~A}-\mathrm{C} 4 \mathrm{~A}$ & $118.6(2)$ & $\mathrm{C} 2 \mathrm{C}-\mathrm{C} 1 \mathrm{C}-\mathrm{C} 7 \mathrm{C}$ & $121.2(2)$ \\
\hline $\mathrm{C} 4 \mathrm{~A}-\mathrm{N} 4 \mathrm{~A}-\mathrm{H} 4 \mathrm{AA}$ & 120.0 & $\mathrm{C} 3 \mathrm{C}-\mathrm{C} 2 \mathrm{C}-\mathrm{C} 1 \mathrm{C}$ & $117.2(2)$ \\
\hline $\mathrm{C} 4 \mathrm{~A}-\mathrm{N} 4 \mathrm{~A}-\mathrm{H} 4 \mathrm{AB}$ & 120.0 & $\mathrm{C} 3 \mathrm{C}-\mathrm{C} 2 \mathrm{C}-\mathrm{C} 8 \mathrm{C}$ & $118.7(2)$ \\
\hline $\mathrm{H} 4 \mathrm{AA}-\mathrm{N} 4 \mathrm{~A}-\mathrm{H} 4 \mathrm{AB}$ & 120.0 & $\mathrm{C} 1 \mathrm{C}-\mathrm{C} 2 \mathrm{C}-\mathrm{C} 8 \mathrm{C}$ & $124.1(2)$ \\
\hline $\mathrm{N} 4 \mathrm{~A}-\mathrm{C} 4 \mathrm{~A}-\mathrm{N} 3 \mathrm{~A}$ & $118.1(2)$ & $\mathrm{C} 4 \mathrm{C}-\mathrm{C} 3 \mathrm{C}-\mathrm{C} 2 \mathrm{C}$ & $121.9(2)$ \\
\hline $\mathrm{N} 4 \mathrm{~A}-\mathrm{C} 4 \mathrm{~A}-\mathrm{C} 5 \mathrm{~A}$ & $123.5(2)$ & $\mathrm{C} 4 \mathrm{C}-\mathrm{C} 3 \mathrm{C}-\mathrm{H} 3 \mathrm{CA}$ & 119.0 \\
\hline $\mathrm{N} 3 \mathrm{~A}-\mathrm{C} 4 \mathrm{~A}-\mathrm{C} 5 \mathrm{~A}$ & $118.4(2)$ & $\mathrm{C} 2 \mathrm{C}-\mathrm{C} 3 \mathrm{C}-\mathrm{H} 3 \mathrm{CA}$ & 119.0 \\
\hline $\mathrm{C} 6 \mathrm{~A}-\mathrm{C} 5 \mathrm{~A}-\mathrm{C} 4 \mathrm{~A}$ & $119.3(2)$ & $\mathrm{C} 3 \mathrm{C}-\mathrm{C} 4 \mathrm{C}-\mathrm{C} 5 \mathrm{C}$ & $120.5(2)$ \\
\hline $\mathrm{C} 6 \mathrm{~A}-\mathrm{C} 5 \mathrm{~A}-\mathrm{C} 11$ & $122.06(19)$ & $\mathrm{C} 3 \mathrm{C}-\mathrm{C} 4 \mathrm{C}-\mathrm{H} 4 \mathrm{CA}$ & 119.8 \\
\hline $\mathrm{C} 4 \mathrm{~A}-\mathrm{C} 5 \mathrm{~A}-\mathrm{Cl1}$ & $118.7(2)$ & $\mathrm{C} 5 \mathrm{C}-\mathrm{C} 4 \mathrm{C}-\mathrm{H} 4 \mathrm{CA}$ & 119.8 \\
\hline $\mathrm{N} 1 \mathrm{~A}-\mathrm{C} 6 \mathrm{~A}-\mathrm{C} 5 \mathrm{~A}$ & $121.2(2)$ & $\mathrm{C} 6 \mathrm{C}-\mathrm{C} 5 \mathrm{C}-\mathrm{C} 4 \mathrm{C}$ & $118.7(3)$ \\
\hline $\mathrm{N} 1 \mathrm{~A}-\mathrm{C} 6 \mathrm{~A}-\mathrm{C} 8 \mathrm{~A}$ & $116.2(2)$ & $\mathrm{C} 6 \mathrm{C}-\mathrm{C} 5 \mathrm{C}-\mathrm{H} 5 \mathrm{CA}$ & 120.7 \\
\hline $\mathrm{C} 5 \mathrm{~A}-\mathrm{C} 6 \mathrm{~A}-\mathrm{C} 8 \mathrm{~A}$ & $122.7(2)$ & $\mathrm{C} 4 \mathrm{C}-\mathrm{C} 5 \mathrm{C}-\mathrm{H} 5 \mathrm{CA}$ & 120.7 \\
\hline $\mathrm{C} 2 \mathrm{~A}-\mathrm{C} 7 \mathrm{~A}-\mathrm{H} 7 \mathrm{AA}$ & 109.5 & $\mathrm{C} 5 \mathrm{C}-\mathrm{C} 6 \mathrm{C}-\mathrm{C} 1 \mathrm{C}$ & $121.2(2)$ \\
\hline $\mathrm{C} 2 \mathrm{~A}-\mathrm{C} 7 \mathrm{~A}-\mathrm{H} 7 \mathrm{AB}$ & 109.5 & $\mathrm{C} 5 \mathrm{C}-\mathrm{C} 6 \mathrm{C}-\mathrm{H} 6 \mathrm{CA}$ & 119.4 \\
\hline $\mathrm{H} 7 \mathrm{AA}-\mathrm{C} 7 \mathrm{~A}-\mathrm{H} 7 \mathrm{AB}$ & 109.5 & $\mathrm{C} 1 \mathrm{C}-\mathrm{C} 6 \mathrm{C}-\mathrm{H} 6 \mathrm{CA}$ & 119.4 \\
\hline $\mathrm{C} 2 \mathrm{~A}-\mathrm{C} 7 \mathrm{~A}-\mathrm{H} 7 \mathrm{AC}$ & 109.5 & $\mathrm{O} 2 \mathrm{C}-\mathrm{C} 7 \mathrm{C}-\mathrm{O} 1 \mathrm{C}$ & $122.8(2)$ \\
\hline H7AA $-\mathrm{C} 7 \mathrm{~A}-\mathrm{H} 7 \mathrm{AC}$ & 109.5 & $\mathrm{O} 2 \mathrm{C}-\mathrm{C} 7 \mathrm{C}-\mathrm{C} 1 \mathrm{C}$ & $124.2(2)$ \\
\hline $\mathrm{H} 7 \mathrm{AB}-\mathrm{C} 7 \mathrm{~A}-\mathrm{H} 7 \mathrm{AC}$ & 109.5 & $\mathrm{O} 1 \mathrm{C}-\mathrm{C} 7 \mathrm{C}-\mathrm{C} 1 \mathrm{C}$ & $112.9(2)$ \\
\hline $\mathrm{C} 6 \mathrm{~A}-\mathrm{C} 8 \mathrm{~A}-\mathrm{H} 8 \mathrm{AA}$ & 109.5 & $\mathrm{C} 2 \mathrm{C}-\mathrm{C} 8 \mathrm{C}-\mathrm{H} 8 \mathrm{CA}$ & 109.5 \\
\hline $\mathrm{C} 6 \mathrm{~A}-\mathrm{C} 8 \mathrm{~A}-\mathrm{H} 8 \mathrm{AB}$ & 109.5 & $\mathrm{C} 2 \mathrm{C}-\mathrm{C} 8 \mathrm{C}-\mathrm{H} 8 \mathrm{CB}$ & 109.5 \\
\hline $\mathrm{H} 8 \mathrm{AA}-\mathrm{C} 8 \mathrm{~A}-\mathrm{H} 8 \mathrm{AB}$ & 109.5 & $\mathrm{H} 8 \mathrm{CA}-\mathrm{C} 8 \mathrm{C}-\mathrm{H} 8 \mathrm{CB}$ & 109.5 \\
\hline $\mathrm{C} 6 \mathrm{~A}-\mathrm{C} 8 \mathrm{~A}-\mathrm{H} 8 \mathrm{AC}$ & 109.5 & $\mathrm{C} 2 \mathrm{C}-\mathrm{C} 8 \mathrm{C}-\mathrm{H} 8 \mathrm{CC}$ & 109.5 \\
\hline $\mathrm{H} 8 \mathrm{AA}-\mathrm{C} 8 \mathrm{~A}-\mathrm{H} 8 \mathrm{AC}$ & 109.5 & $\mathrm{H} 8 \mathrm{CA}-\mathrm{C} 8 \mathrm{C}-\mathrm{H} 8 \mathrm{CC}$ & 109.5 \\
\hline $\mathrm{H} 8 \mathrm{AB}-\mathrm{C} 8 \mathrm{~A}-\mathrm{H} 8 \mathrm{AC}$ & 109.5 & $\mathrm{H} 8 \mathrm{CB}-\mathrm{C} 8 \mathrm{C}-\mathrm{H} 8 \mathrm{CC}$ & 109.5 \\
\hline $\mathrm{C} 2 \mathrm{~B}-\mathrm{N} 1 \mathrm{~B}-\mathrm{C} 6 \mathrm{~B}$ & $116.8(2)$ & C7D-O1D-H1D & $110(3)$ \\
\hline $\mathrm{N} 1 \mathrm{~B}-\mathrm{C} 2 \mathrm{~B}-\mathrm{N} 3 \mathrm{~B}$ & $126.3(2)$ & $\mathrm{C} 6 \mathrm{D}-\mathrm{C} 1 \mathrm{D}-\mathrm{C} 2 \mathrm{D}$ & $120.2(2)$ \\
\hline $\mathrm{N} 1 \mathrm{~B}-\mathrm{C} 2 \mathrm{~B}-\mathrm{C} 7 \mathrm{~B}$ & $117.6(2)$ & $\mathrm{C} 6 \mathrm{D}-\mathrm{C} 1 \mathrm{D}-\mathrm{C} 7 \mathrm{D}$ & $118.4(2)$ \\
\hline $\mathrm{N} 3 \mathrm{~B}-\mathrm{C} 2 \mathrm{~B}-\mathrm{C} 7 \mathrm{~B}$ & $116.1(2)$ & $\mathrm{C} 2 \mathrm{D}-\mathrm{C} 1 \mathrm{D}-\mathrm{C} 7 \mathrm{D}$ & $121.3(2)$ \\
\hline $\mathrm{C} 2 \mathrm{~B}-\mathrm{N} 3 \mathrm{~B}-\mathrm{C} 4 \mathrm{~B}$ & $118.0(2)$ & $\mathrm{C} 3 \mathrm{D}-\mathrm{C} 2 \mathrm{D}-\mathrm{C} 1 \mathrm{D}$ & $117.4(2)$ \\
\hline $\mathrm{C} 4 \mathrm{~B}-\mathrm{N} 4 \mathrm{~B}-\mathrm{H} 4 \mathrm{BA}$ & 120.0 & $\mathrm{C} 3 \mathrm{D}-\mathrm{C} 2 \mathrm{D}-\mathrm{C} 8 \mathrm{D}$ & $118.6(2)$ \\
\hline $\mathrm{C} 4 \mathrm{~B}-\mathrm{N} 4 \mathrm{~B}-\mathrm{H} 4 \mathrm{BB}$ & 120.0 & $\mathrm{C} 1 \mathrm{D}-\mathrm{C} 2 \mathrm{D}-\mathrm{C} 8 \mathrm{D}$ & $124.0(2)$ \\
\hline $\mathrm{H} 4 \mathrm{BA}-\mathrm{N} 4 \mathrm{~B}-\mathrm{H} 4 \mathrm{BB}$ & 120.0 & $\mathrm{C} 4 \mathrm{D}-\mathrm{C} 3 \mathrm{D}-\mathrm{C} 2 \mathrm{D}$ & $122.0(2)$ \\
\hline $\mathrm{N} 4 \mathrm{~B}-\mathrm{C} 4 \mathrm{~B}-\mathrm{N} 3 \mathrm{~B}$ & $117.7(2)$ & $\mathrm{C} 4 \mathrm{D}-\mathrm{C} 3 \mathrm{D}-\mathrm{H} 3 \mathrm{DA}$ & 119.0 \\
\hline $\mathrm{N} 4 \mathrm{~B}-\mathrm{C} 4 \mathrm{~B}-\mathrm{C} 5 \mathrm{~B}$ & $123.6(2)$ & $\mathrm{C} 2 \mathrm{D}-\mathrm{C} 3 \mathrm{D}-\mathrm{H} 3 \mathrm{DA}$ & 119.0 \\
\hline $\mathrm{N} 3 \mathrm{~B}-\mathrm{C} 4 \mathrm{~B}-\mathrm{C} 5 \mathrm{~B}$ & $118.7(2)$ & $\mathrm{C} 3 \mathrm{D}-\mathrm{C} 4 \mathrm{D}-\mathrm{C} 5 \mathrm{D}$ & $120.2(2)$ \\
\hline $\mathrm{C} 6 \mathrm{~B}-\mathrm{C} 5 \mathrm{~B}-\mathrm{C} 4 \mathrm{~B}$ & $119.7(2)$ & $\mathrm{C} 3 \mathrm{D}-\mathrm{C} 4 \mathrm{D}-\mathrm{H} 4 \mathrm{DA}$ & 119.9 \\
\hline $\mathrm{C} 6 \mathrm{~B}-\mathrm{C} 5 \mathrm{~B}-\mathrm{Cl} 2$ & $121.73(19)$ & $\mathrm{C} 5 \mathrm{D}-\mathrm{C} 4 \mathrm{D}-\mathrm{H} 4 \mathrm{DA}$ & 119.9 \\
\hline
\end{tabular}




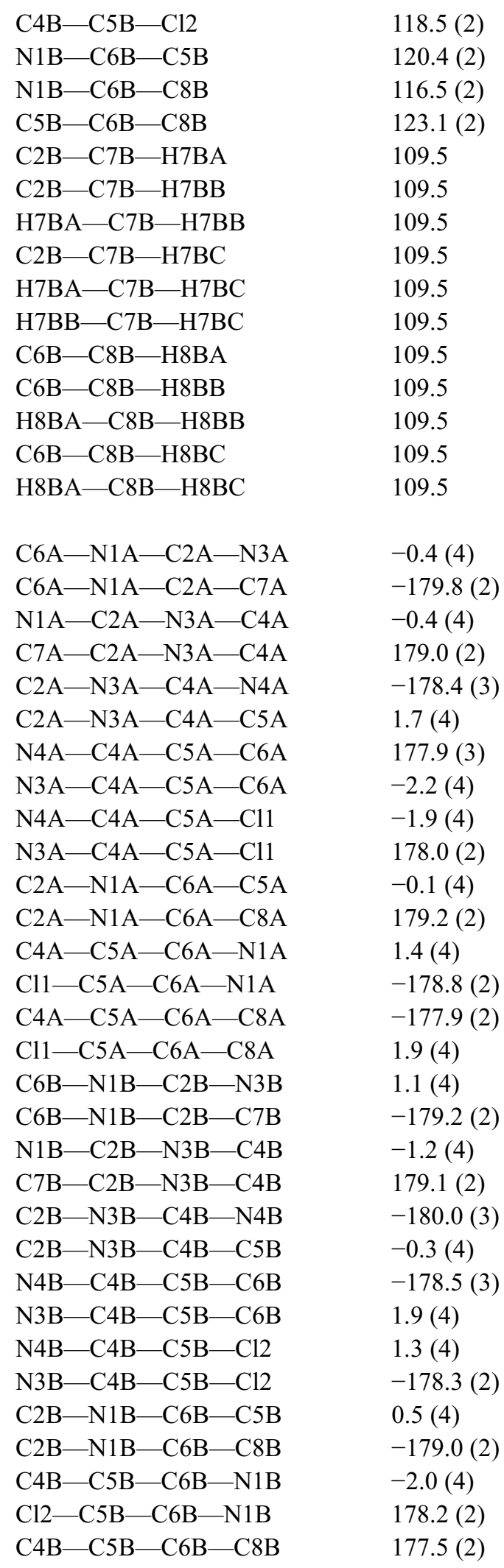

$118.5(2)$

$120.4(2)$

$116.5(2)$

$123.1(2)$

109.5

109.5

109.5

109.5

109.5

109.5

109.5

109.5

109.5

109.5

109.5

$-0.4(4)$

$-179.8(2)$

$-0.4(4)$

$179.0(2)$

$-178.4(3)$

$1.7(4)$

$177.9(3)$

$-2.2(4)$

$-1.9(4)$

$178.0(2)$

-0.1 (4)

$179.2(2)$

$1.4(4)$

$-178.8(2)$

$-177.9(2)$

$1.9(4)$

1.1 (4)

$-179.2(2)$

$-1.2(4)$

$179.1(2)$

$-180.0(3)$

$-0.3(4)$

$-178.5(3)$

1.9 (4)

$1.3(4)$

$-178.3(2)$

$0.5(4)$

$-179.0(2)$

$-2.0(4)$

$178.2(2)$

$177.5(2)$

$\mathrm{C} 6 \mathrm{D}-\mathrm{C} 5 \mathrm{D}-\mathrm{C} 4 \mathrm{D}$

C6D-C5D-H5DA

$\mathrm{C} 4 \mathrm{D}-\mathrm{C} 5 \mathrm{D}-\mathrm{H} 5 \mathrm{DA}$

C5D-C6D-C1D

$\mathrm{C} 5 \mathrm{D}-\mathrm{C} 6 \mathrm{D}-\mathrm{H} 6 \mathrm{DA}$

C1D - C6D-H6DA

O2D-C7D-O1D

O2D $-C 7 D-C 1 D$

O1D $-C 7 D-C 1 D$

C2D-C8D-H8DA

$\mathrm{C} 2 \mathrm{D}-\mathrm{C} 8 \mathrm{D}-\mathrm{H} 8 \mathrm{DB}$

$\mathrm{H} 8 \mathrm{DA}-\mathrm{C} 8 \mathrm{D}-\mathrm{H} 8 \mathrm{DB}$

$\mathrm{C} 2 \mathrm{D}-\mathrm{C} 8 \mathrm{D}-\mathrm{H} 8 \mathrm{DC}$

H8DA-C8D-H8DC

$\mathrm{H} 8 \mathrm{DB}-\mathrm{C} 8 \mathrm{D}-\mathrm{H} 8 \mathrm{DC}$

$\mathrm{Cl} 2-\mathrm{C} 5 \mathrm{~B}-\mathrm{C} 6 \mathrm{~B}-\mathrm{C} 8 \mathrm{~B}$

$\mathrm{C} 6 \mathrm{C}-\mathrm{C} 1 \mathrm{C}-\mathrm{C} 2 \mathrm{C}-\mathrm{C} 3 \mathrm{C}$

$\mathrm{C} 7 \mathrm{C}-\mathrm{C} 1 \mathrm{C}-\mathrm{C} 2 \mathrm{C}-\mathrm{C} 3 \mathrm{C}$

$\mathrm{C} 6 \mathrm{C}-\mathrm{C} 1 \mathrm{C}-\mathrm{C} 2 \mathrm{C}-\mathrm{C} 8 \mathrm{C}$

$\mathrm{C} 7 \mathrm{C}-\mathrm{C} 1 \mathrm{C}-\mathrm{C} 2 \mathrm{C}-\mathrm{C} 8 \mathrm{C}$

$\mathrm{C} 1 \mathrm{C}-\mathrm{C} 2 \mathrm{C}-\mathrm{C} 3 \mathrm{C}-\mathrm{C} 4 \mathrm{C}$

$\mathrm{C} 8 \mathrm{C}-\mathrm{C} 2 \mathrm{C}-\mathrm{C} 3 \mathrm{C}-\mathrm{C} 4 \mathrm{C}$

$\mathrm{C} 2 \mathrm{C}-\mathrm{C} 3 \mathrm{C}-\mathrm{C} 4 \mathrm{C}-\mathrm{C} 5 \mathrm{C}$

$\mathrm{C} 3 \mathrm{C}-\mathrm{C} 4 \mathrm{C}-\mathrm{C} 5 \mathrm{C}-\mathrm{C} 6 \mathrm{C}$

$\mathrm{C} 4 \mathrm{C}-\mathrm{C} 5 \mathrm{C}-\mathrm{C} 6 \mathrm{C}-\mathrm{C} 1 \mathrm{C}$

$\mathrm{C} 2 \mathrm{C}-\mathrm{C} 1 \mathrm{C}-\mathrm{C} 6 \mathrm{C}-\mathrm{C} 5 \mathrm{C}$

$\mathrm{C} 7 \mathrm{C}-\mathrm{C} 1 \mathrm{C}-\mathrm{C} 6 \mathrm{C}-\mathrm{C} 5 \mathrm{C}$

$\mathrm{C} 6 \mathrm{C}-\mathrm{C} 1 \mathrm{C}-\mathrm{C} 7 \mathrm{C}-\mathrm{O} 2 \mathrm{C}$

$\mathrm{C} 2 \mathrm{C}-\mathrm{C} 1 \mathrm{C}-\mathrm{C} 7 \mathrm{C}-\mathrm{O} 2 \mathrm{C}$

$\mathrm{C} 6 \mathrm{C}-\mathrm{C} 1 \mathrm{C}-\mathrm{C} 7 \mathrm{C}-\mathrm{O} 1 \mathrm{C}$

$\mathrm{C} 2 \mathrm{C}-\mathrm{C} 1 \mathrm{C}-\mathrm{C} 7 \mathrm{C}-\mathrm{O} 1 \mathrm{C}$

$\mathrm{C} 6 \mathrm{D}-\mathrm{C} 1 \mathrm{D}-\mathrm{C} 2 \mathrm{D}-\mathrm{C} 3 \mathrm{D}$

$\mathrm{C} 7 \mathrm{D}-\mathrm{C} 1 \mathrm{D}-\mathrm{C} 2 \mathrm{D}-\mathrm{C} 3 \mathrm{D}$

$\mathrm{C} 6 \mathrm{D}-\mathrm{C} 1 \mathrm{D}-\mathrm{C} 2 \mathrm{D}-\mathrm{C} 8 \mathrm{D}$

$\mathrm{C} 7 \mathrm{D}-\mathrm{C} 1 \mathrm{D}-\mathrm{C} 2 \mathrm{D}-\mathrm{C} 8 \mathrm{D}$

$\mathrm{C} 1 \mathrm{D}-\mathrm{C} 2 \mathrm{D}-\mathrm{C} 3 \mathrm{D}-\mathrm{C} 4 \mathrm{D}$

$\mathrm{C} 8 \mathrm{D}-\mathrm{C} 2 \mathrm{D}-\mathrm{C} 3 \mathrm{D}-\mathrm{C} 4 \mathrm{D}$

$\mathrm{C} 2 \mathrm{D}-\mathrm{C} 3 \mathrm{D}-\mathrm{C} 4 \mathrm{D}-\mathrm{C} 5 \mathrm{D}$

$\mathrm{C} 3 \mathrm{D}-\mathrm{C} 4 \mathrm{D}-\mathrm{C} 5 \mathrm{D}-\mathrm{C} 6 \mathrm{D}$

$\mathrm{C} 4 \mathrm{D}-\mathrm{C} 5 \mathrm{D}-\mathrm{C} 6 \mathrm{D}-\mathrm{C} 1 \mathrm{D}$

$\mathrm{C} 2 \mathrm{D}-\mathrm{C} 1 \mathrm{D}-\mathrm{C} 6 \mathrm{D}-\mathrm{C} 5 \mathrm{D}$

C7D $-C 1 D-C 6 D-C 5 D$

$\mathrm{C} 6 \mathrm{D}-\mathrm{C} 1 \mathrm{D}-\mathrm{C} 7 \mathrm{D}-\mathrm{O} 2 \mathrm{D}$

$\mathrm{C} 2 \mathrm{D}-\mathrm{C} 1 \mathrm{D}-\mathrm{C} 7 \mathrm{D}-\mathrm{O} 2 \mathrm{D}$

$\mathrm{C} 6 \mathrm{D}-\mathrm{C} 1 \mathrm{D}-\mathrm{C} 7 \mathrm{D}-\mathrm{O} 1 \mathrm{D}$

$\mathrm{C} 2 \mathrm{D}-\mathrm{C} 1 \mathrm{D}-\mathrm{C} 7 \mathrm{D}-\mathrm{O} 1 \mathrm{D}$
$119.3(3)$

120.4

120.4

$120.8(2)$

119.6

119.6

$122.1(2)$

124.7 (2)

113.2 (2)

109.5

109.5

109.5

109.5

109.5

109.5

$-2.3(4)$

0.5 (4)

-177.4 (2)

-178.8 (3)

3.4 (4)

0.9 (4)

-179.8 (3)

-1.1 (4)

-0.1 (4)

1.5 (4)

-1.7 (4)

176.2 (3)

$-159.6(3)$

18.3 (4)

18.1 (4)

-164.0 (3)

3.6 (4)

-176.8 (2)

$-177.2(3)$

2.4 (4)

-2.9 (4)

$177.8(3)$

$0.8(4)$

0.8 (4)

-0.1 (4)

-2.2 (4)

178.2 (2)

$162.9(3)$

-16.7 (4)

$-16.7(4)$

$163.7(3)$ 
Hydrogen-bond geometry $\left(\AA,{ }^{\circ}\right)$

\begin{tabular}{lllll}
\hline$D-\mathrm{H} \cdots A$ & $D-\mathrm{H}$ & $\mathrm{H} \cdots A$ & $D \cdots A$ & $D-\mathrm{H} \cdots A$ \\
\hline $\mathrm{N} 4 A-\mathrm{H} 4 A A \cdots \mathrm{O} 2 C$ & 0.88 & 2.07 & $2.938(3)$ & 169 \\
$\mathrm{~N} 4 A-\mathrm{H} 4 A B \cdots \mathrm{N} 1 B$ & 0.88 & 2.39 & $3.104(3)$ & 139 \\
$\mathrm{C} 7 A-\mathrm{H} 7 A A \cdots \mathrm{Cl} 2^{\mathrm{i}}$ & 0.98 & 2.84 & $3.816(3)$ & 173 \\
$\mathrm{C} 7 A-\mathrm{H} 7 A B \cdots \mathrm{O} 1 C$ & 0.98 & 2.54 & $3.281(3)$ & 132 \\
$\mathrm{~N} 4 B-\mathrm{H} 4 B A \cdots \mathrm{O} 2 D$ & 0.88 & 2.07 & $2.938(3)$ & 170 \\
$\mathrm{~N} 4 B-\mathrm{H} 4 B B \cdots \mathrm{N} 1 A^{\mathrm{ii}}$ & 0.88 & 2.42 & $3.164(3)$ & 142 \\
$\mathrm{C} 7 B-\mathrm{H} 7 B A \cdots \mathrm{C} 11$ & 0.98 & 2.94 & $3.783(3)$ & 145 \\
$\mathrm{O} 1 C-\mathrm{H} 1 C \cdots \mathrm{N} 3 A$ & $0.96(5)$ & $1.70(5)$ & $2.662(3)$ & $174(5)$ \\
$\mathrm{O} 1 D-\mathrm{H} 1 D \cdots \mathrm{N} 3 B$ & $0.95(4)$ & $1.75(4)$ & $2.685(3)$ & $170(4)$ \\
\hline
\end{tabular}

Symmetry codes: (i) $x, y+1, z$; (ii) $x, y-1, z$.

4-Amino-5-chloro-2,6-dimethylpyrimidine-3-methylbenzoic acid (1/1) (COCRYSTAL-8)

Crystal data

$\mathrm{C}_{6} \mathrm{H}_{8} \mathrm{ClN}_{3} \cdot \mathrm{C}_{8} \mathrm{H}_{8} \mathrm{O}_{2}$

$M_{r}=293.75$

Orthorhombic, $\mathrm{Pbcn}$

$a=8.4524$ (1) $\AA$

$b=13.5387(2) \AA$

$c=24.9813(3) \AA$

$V=2858.72(6) \AA^{3}$

$Z=8$

$F(000)=1232$

Data collection

Agilent SuperNova Dual Source diffractometer with an Atlas detector

Radiation source: sealed X-ray tube

Detector resolution: 10.6501 pixels $\mathrm{mm}^{-1}$

$\omega$ scans

Absorption correction: multi-scan

(CrysAlis PRO; Agilent, 2014)

$T_{\min }=0.680, T_{\max }=1.000$

Refinement

Refinement on $F^{2}$

Least-squares matrix: full

$R\left[F^{2}>2 \sigma\left(F^{2}\right)\right]=0.037$

$w R\left(F^{2}\right)=0.103$

$S=1.04$

3006 reflections

196 parameters

0 restraints
$D_{\mathrm{x}}=1.365 \mathrm{Mg} \mathrm{m}^{-3}$

$\mathrm{Cu} K \alpha$ radiation, $\lambda=1.54184 \AA$

Cell parameters from 15282 reflections

$\theta=3.6-76.6^{\circ}$

$\mu=2.42 \mathrm{~mm}^{-1}$

$T=120 \mathrm{~K}$

Prism, colorless

$0.42 \times 0.24 \times 0.19 \mathrm{~mm}$

26401 measured reflections

3006 independent reflections

2904 reflections with $I>2 \sigma(I)$

$R_{\text {int }}=0.033$

$\theta_{\text {max }}=76.8^{\circ}, \theta_{\min }=3.5^{\circ}$

$h=-7 \rightarrow 10$

$k=-15 \rightarrow 16$

$l=-30 \rightarrow 31$

Hydrogen site location: mixed

$\mathrm{H}$ atoms treated by a mixture of independent and constrained refinement

$w=1 /\left[\sigma^{2}\left(F_{\mathrm{o}}^{2}\right)+(0.0616 P)^{2}+1.3483 P\right]$

where $P=\left(F_{\mathrm{o}}{ }^{2}+2 F_{\mathrm{c}}{ }^{2}\right) / 3$

$(\Delta / \sigma)_{\max }=0.001$

$\Delta \rho_{\max }=0.30$ e $\AA^{-3}$

$\Delta \rho_{\min }=-0.31$ e $\AA^{-3}$

Special details

Geometry. All esds (except the esd in the dihedral angle between two 1.s. planes) are estimated using the full covariance matrix. The cell esds are taken into account individually in the estimation of esds in distances, angles and torsion angles; correlations between esds in cell parameters are only used when they are defined by crystal symmetry. An approximate (isotropic) treatment of cell esds is used for estimating esds involving l.s. planes. 
Fractional atomic coordinates and isotropic or equivalent isotropic displacement parameters $\left(\AA^{2}\right)$

\begin{tabular}{|c|c|c|c|c|}
\hline & $x$ & $y$ & $z$ & $U_{\text {iso }} * / U_{\text {eq }}$ \\
\hline $\mathrm{Cl1}$ & $0.15056(4)$ & $0.43229(2)$ & $0.19457(2)$ & $0.02742(13)$ \\
\hline N1A & $0.27927(13)$ & $0.68415(8)$ & $0.26427(4)$ & $0.0221(2)$ \\
\hline $\mathrm{C} 2 \mathrm{~A}$ & $0.35774(15)$ & $0.64739(10)$ & $0.30611(5)$ & 0.0203 \\
\hline N3A & $0.38017(13)$ & $0.55115(8)$ & $0.31659(4)$ & $0.0201(2)$ \\
\hline N4A & $0.33933(14)$ & $0.38852(9)$ & $0.29368(5)$ & 0.0238 \\
\hline H41 & $0.391(2)$ & $0.3742(13)$ & $0.3208(7)$ & $0.024(4)^{*}$ \\
\hline $\mathrm{H} 42$ & $0.297(2)$ & $0.3408(15)$ & $0.2732(8)$ & $0.041(5)^{*}$ \\
\hline $\mathrm{C} 4 \mathrm{~A}$ & $0.31709(15)$ & $0.48386(9)$ & $0.28280(5)$ & 0.0196 \\
\hline C5A & $0.23205(15)$ & $0.51817(9)$ & $0.23781(5)$ & 0.0208 \\
\hline C6A & $0.21632(15)$ & $0.61794(10)$ & $0.22941(5)$ & 0.0218 \\
\hline C7A & $0.42722(17)$ & $0.71901(9)$ & $0.34549(5)$ & $0.0272(3)$ \\
\hline H7AA & 0.4024 & 0.7867 & 0.3343 & $0.041 *$ \\
\hline H7AB & 0.5423 & 0.7104 & 0.3468 & $0.041 *$ \\
\hline H7AC & 0.3824 & 0.7068 & 0.3810 & $0.041 *$ \\
\hline $\mathrm{C} 8 \mathrm{~A}$ & $0.13053(17)$ & $0.65888(11)$ & $0.18186(6)$ & 0.0292 \\
\hline H8AA & 0.1421 & 0.7309 & 0.1812 & $0.044^{*}$ \\
\hline H8AB & 0.0181 & 0.6418 & 0.1843 & $0.044 *$ \\
\hline H8AC & 0.1752 & 0.6308 & 0.1490 & $0.044^{*}$ \\
\hline O1B & $0.56542(12)$ & $0.51547(7)$ & $0.40098(4)$ & $0.0260(2)$ \\
\hline H1B & $0.502(3)$ & $0.5220(17)$ & $0.3730(9)$ & $0.056(6)^{*}$ \\
\hline $\mathrm{O} 2 \mathrm{~B}$ & $0.51805(11)$ & $0.35371(7)$ & $0.39001(4)$ & $0.0259(2)$ \\
\hline C1B & $0.68785(15)$ & $0.40432(10)$ & $0.46069(5)$ & 0.0218 \\
\hline $\mathrm{C} 2 \mathrm{~B}$ & $0.73386(15)$ & $0.48174(10)$ & $0.49402(5)$ & 0.0231 \\
\hline $\mathrm{H} 2 \mathrm{BA}$ & 0.6996 & 0.5470 & 0.4863 & $0.028 *$ \\
\hline $\mathrm{C} 3 \mathrm{~B}$ & $0.82943(16)$ & $0.46448(11)$ & $0.53845(5)$ & 0.0254 \\
\hline C4B & $0.87966(17)$ & $0.36796(11)$ & $0.54822(6)$ & $0.0296(3)$ \\
\hline H4BA & 0.9452 & 0.3549 & 0.5783 & $0.035^{*}$ \\
\hline $\mathrm{C} 5 \mathrm{~B}$ & $0.83573(18)$ & $0.29079(11)$ & $0.51486(6)$ & $0.0324(3)$ \\
\hline H5BA & 0.8722 & 0.2257 & 0.5220 & $0.039 *$ \\
\hline C6B & $0.73882(17)$ & $0.30830(10)$ & $0.47111(6)$ & $0.0277(3)$ \\
\hline H6BA & 0.7075 & 0.2554 & 0.4485 & $0.033^{*}$ \\
\hline $\mathrm{C} 7 \mathrm{~B}$ & $0.58274(15)$ & $0.42178(9)$ & $0.41389(5)$ & 0.0213 \\
\hline C8B & $0.87751(18)$ & $0.54831(12)$ & $0.57482(6)$ & $0.0312(3)$ \\
\hline H8BA & 0.9427 & 0.5226 & 0.6041 & $0.047 *$ \\
\hline H8BB & 0.7827 & 0.5798 & 0.5896 & $0.047^{*}$ \\
\hline $\mathrm{H} 8 \mathrm{BC}$ & 0.9384 & 0.5970 & 0.5544 & $0.047 *$ \\
\hline
\end{tabular}

Atomic displacement parameters $\left(\AA^{2}\right)$

\begin{tabular}{lllllll}
\hline & $U^{11}$ & $U^{22}$ & $U^{33}$ & $U^{12}$ & $U^{13}$ & $U^{23}$ \\
\hline C11 & $0.0331(2)$ & $0.0262(2)$ & $0.02299(19)$ & $-0.00227(12)$ & $-0.00619(12)$ & $-0.00403(11)$ \\
N1A & $0.0231(5)$ & $0.0199(5)$ & $0.0233(5)$ & $0.0012(4)$ & $0.0003(4)$ & $0.0013(4)$ \\
C2A & $0.0201(6)$ & $0.0183(6)$ & $0.0225(6)$ & $-0.0007(4)$ & $0.0018(4)$ & $0.0009(4)$ \\
N3A & $0.0214(5)$ & $0.0187(5)$ & $0.0203(5)$ & $-0.0009(4)$ & $-0.0010(4)$ & $0.0003(4)$ \\
N4A & $0.0306(6)$ & $0.0184(6)$ & $0.0225(6)$ & $-0.0004(4)$ & $-0.0057(5)$ & $0.0001(4)$
\end{tabular}




$\begin{array}{lllllll}\text { C4A } & 0.0195(5) & 0.0194(6) & 0.0200(6) & -0.0012(5) & 0.0031(5) & -0.0003(4) \\ \text { C5A } & 0.0205(6) & 0.0227(6) & 0.0193(6) & -0.0011(5) & 0.0001(5) & -0.0018(4) \\ \text { C6A } & 0.0197(6) & 0.0252(6) & 0.0206(6) & 0.0016(5) & 0.0012(5) & 0.0012(5) \\ \text { C7A } & 0.0323(7) & 0.0204(6) & 0.0290(7) & -0.0019(5) & -0.0043(5) & -0.0012(5) \\ \text { C8A } & 0.0313(7) & 0.0290(7) & 0.0271(7) & 0.0044(6) & -0.0052(6) & 0.0044(6) \\ \text { O1B } & 0.0317(5) & 0.0204(5) & 0.0260(5) & 0.0007(4) & -0.0091(4) & 0.0009(4) \\ \text { O2B } & 0.0292(5) & 0.0226(5) & 0.0259(5) & -0.0029(4) & -0.0062(4) & 0.0006(3) \\ \text { C1B } & 0.0189(6) & 0.0249(6) & 0.0217(6) & -0.0010(5) & -0.0001(5) & 0.0023(5) \\ \text { C2B } & 0.0208(6) & 0.0257(7) & 0.0229(6) & 0.0008(5) & 0.0005(5) & -0.0003(5) \\ \text { C3B } & 0.0206(6) & 0.0336(7) & 0.0222(6) & -0.0019(5) & 0.0005(5) & -0.0006(5) \\ \text { C4B } & 0.0250(6) & 0.0359(8) & 0.0278(7) & -0.0007(6) & -0.0064(5) & 0.0060(6) \\ \text { C5B } & 0.0320(7) & 0.0262(7) & 0.0390(8) & 0.0002(6) & -0.0101(6) & 0.0074(6) \\ \text { C6B } & 0.0265(7) & 0.0244(7) & 0.0323(7) & -0.0022(5) & -0.0059(5) & 0.0023(5) \\ \text { C7B } & 0.0198(6) & 0.0227(6) & 0.0214(6) & 0.0005(5) & 0.0013(5) & 0.0009(5) \\ \text { C8B } & 0.0287(7) & 0.0394(8) & 0.0254(7) & -0.0002(6) & -0.0042(5) & -0.0063(6)\end{array}$

Geometric parameters $\left(\AA,{ }^{\circ}\right)$

\begin{tabular}{|c|c|c|c|}
\hline $\mathrm{Cl1}-\mathrm{C} 5 \mathrm{~A}$ & $1.7301(13)$ & $\mathrm{O} 1 \mathrm{~B}-\mathrm{C} 7 \mathrm{~B}$ & $1.3169(16)$ \\
\hline $\mathrm{N} 1 \mathrm{~A}-\mathrm{C} 2 \mathrm{~A}$ & $1.3342(17)$ & $\mathrm{O} 1 \mathrm{~B}-\mathrm{H} 1 \mathrm{~B}$ & $0.88(2)$ \\
\hline $\mathrm{N} 1 \mathrm{~A}-\mathrm{C} 6 \mathrm{~A}$ & $1.3584(17)$ & $\mathrm{O} 2 \mathrm{~B}-\mathrm{C} 7 \mathrm{~B}$ & $1.2264(16)$ \\
\hline $\mathrm{C} 2 \mathrm{~A}-\mathrm{N} 3 \mathrm{~A}$ & $1.3424(17)$ & $\mathrm{C} 1 \mathrm{~B}-\mathrm{C} 6 \mathrm{~B}$ & $1.3940(18)$ \\
\hline $\mathrm{C} 2 \mathrm{~A}-\mathrm{C} 7 \mathrm{~A}$ & $1.5011(18)$ & $\mathrm{C} 1 \mathrm{~B}-\mathrm{C} 2 \mathrm{~B}$ & $1.3940(18)$ \\
\hline $\mathrm{N} 3 \mathrm{~A}-\mathrm{C} 4 \mathrm{~A}$ & $1.3516(16)$ & $\mathrm{C} 1 \mathrm{~B}-\mathrm{C} 7 \mathrm{~B}$ & $1.4874(18)$ \\
\hline $\mathrm{N} 4 \mathrm{~A}-\mathrm{C} 4 \mathrm{~A}$ & $1.3325(17)$ & $\mathrm{C} 2 \mathrm{~B}-\mathrm{C} 3 \mathrm{~B}$ & $1.3924(18)$ \\
\hline $\mathrm{N} 4 \mathrm{~A}-\mathrm{H} 41$ & $0.829(18)$ & $\mathrm{C} 2 \mathrm{~B}-\mathrm{H} 2 \mathrm{BA}$ & 0.9500 \\
\hline $\mathrm{N} 4 \mathrm{~A}-\mathrm{H} 42$ & $0.90(2)$ & $\mathrm{C} 3 \mathrm{~B}-\mathrm{C} 4 \mathrm{~B}$ & $1.396(2)$ \\
\hline $\mathrm{C} 4 \mathrm{~A}-\mathrm{C} 5 \mathrm{~A}$ & $1.4125(18)$ & $\mathrm{C} 3 \mathrm{~B}-\mathrm{C} 8 \mathrm{~B}$ & $1.510(2)$ \\
\hline $\mathrm{C} 5 \mathrm{~A}-\mathrm{C} 6 \mathrm{~A}$ & $1.3735(19)$ & $\mathrm{C} 4 \mathrm{~B}-\mathrm{C} 5 \mathrm{~B}$ & $1.387(2)$ \\
\hline $\mathrm{C} 6 \mathrm{~A}-\mathrm{C} 8 \mathrm{~A}$ & $1.4979(18)$ & $\mathrm{C} 4 \mathrm{~B}-\mathrm{H} 4 \mathrm{BA}$ & 0.9500 \\
\hline C7A-H7AA & 0.9800 & $\mathrm{C} 5 \mathrm{~B}-\mathrm{C} 6 \mathrm{~B}$ & $1.386(2)$ \\
\hline $\mathrm{C} 7 \mathrm{~A}-\mathrm{H} 7 \mathrm{AB}$ & 0.9800 & $\mathrm{C} 5 \mathrm{~B}-\mathrm{H} 5 \mathrm{BA}$ & 0.9500 \\
\hline $\mathrm{C} 7 \mathrm{~A}-\mathrm{H} 7 \mathrm{AC}$ & 0.9800 & C6B-H6BA & 0.9500 \\
\hline $\mathrm{C} 8 \mathrm{~A}-\mathrm{H} 8 \mathrm{AA}$ & 0.9800 & $\mathrm{C} 8 \mathrm{~B}-\mathrm{H} 8 \mathrm{BA}$ & 0.9800 \\
\hline $\mathrm{C} 8 \mathrm{~A}-\mathrm{H} 8 \mathrm{AB}$ & 0.9800 & $\mathrm{C} 8 \mathrm{~B}-\mathrm{H} 8 \mathrm{BB}$ & 0.9800 \\
\hline $\mathrm{C} 8 \mathrm{~A}-\mathrm{H} 8 \mathrm{AC}$ & 0.9800 & $\mathrm{C} 8 \mathrm{~B}-\mathrm{H} 8 \mathrm{BC}$ & 0.9800 \\
\hline $\mathrm{C} 2 \mathrm{~A}-\mathrm{N} 1 \mathrm{~A}-\mathrm{C} 6 \mathrm{~A}$ & $116.79(11)$ & $\mathrm{C} 7 \mathrm{~B}-\mathrm{O} 1 \mathrm{~B}-\mathrm{H} 1 \mathrm{~B}$ & $110.9(15)$ \\
\hline $\mathrm{N} 1 \mathrm{~A}-\mathrm{C} 2 \mathrm{~A}-\mathrm{N} 3 \mathrm{~A}$ & $125.81(12)$ & $\mathrm{C} 6 \mathrm{~B}-\mathrm{C} 1 \mathrm{~B}-\mathrm{C} 2 \mathrm{~B}$ & $120.23(12)$ \\
\hline $\mathrm{N} 1 \mathrm{~A}-\mathrm{C} 2 \mathrm{~A}-\mathrm{C} 7 \mathrm{~A}$ & $117.83(12)$ & $\mathrm{C} 6 \mathrm{~B}-\mathrm{C} 1 \mathrm{~B}-\mathrm{C} 7 \mathrm{~B}$ & $118.66(12)$ \\
\hline $\mathrm{N} 3 \mathrm{~A}-\mathrm{C} 2 \mathrm{~A}-\mathrm{C} 7 \mathrm{~A}$ & $116.35(11)$ & $\mathrm{C} 2 \mathrm{~B}-\mathrm{C} 1 \mathrm{~B}-\mathrm{C} 7 \mathrm{~B}$ & $121.11(12)$ \\
\hline $\mathrm{C} 2 \mathrm{~A}-\mathrm{N} 3 \mathrm{~A}-\mathrm{C} 4 \mathrm{~A}$ & $118.47(11)$ & $\mathrm{C} 3 \mathrm{~B}-\mathrm{C} 2 \mathrm{~B}-\mathrm{C} 1 \mathrm{~B}$ & $120.78(13)$ \\
\hline $\mathrm{C} 4 \mathrm{~A}-\mathrm{N} 4 \mathrm{~A}-\mathrm{H} 41$ & $117.9(12)$ & $\mathrm{C} 3 \mathrm{~B}-\mathrm{C} 2 \mathrm{~B}-\mathrm{H} 2 \mathrm{BA}$ & 119.6 \\
\hline $\mathrm{C} 4 \mathrm{~A}-\mathrm{N} 4 \mathrm{~A}-\mathrm{H} 42$ & $121.6(13)$ & $\mathrm{C} 1 \mathrm{~B}-\mathrm{C} 2 \mathrm{~B}-\mathrm{H} 2 \mathrm{BA}$ & 119.6 \\
\hline $\mathrm{H} 41-\mathrm{N} 4 \mathrm{~A}-\mathrm{H} 42$ & $120.5(18)$ & $\mathrm{C} 2 \mathrm{~B}-\mathrm{C} 3 \mathrm{~B}-\mathrm{C} 4 \mathrm{~B}$ & $118.23(13)$ \\
\hline $\mathrm{N} 4 \mathrm{~A}-\mathrm{C} 4 \mathrm{~A}-\mathrm{N} 3 \mathrm{~A}$ & $118.03(12)$ & $\mathrm{C} 2 \mathrm{~B}-\mathrm{C} 3 \mathrm{~B}-\mathrm{C} 8 \mathrm{~B}$ & $120.65(14)$ \\
\hline $\mathrm{N} 4 \mathrm{~A}-\mathrm{C} 4 \mathrm{~A}-\mathrm{C} 5 \mathrm{~A}$ & $123.55(12)$ & $\mathrm{C} 4 \mathrm{~B}-\mathrm{C} 3 \mathrm{~B}-\mathrm{C} 8 \mathrm{~B}$ & $121.12(13)$ \\
\hline $\mathrm{N} 3 \mathrm{~A}-\mathrm{C} 4 \mathrm{~A}-\mathrm{C} 5 \mathrm{~A}$ & $118.42(11)$ & $\mathrm{C} 5 \mathrm{~B}-\mathrm{C} 4 \mathrm{~B}-\mathrm{C} 3 \mathrm{~B}$ & $121.25(13)$ \\
\hline
\end{tabular}




$\begin{array}{ll}\text { C6A-C5A-C4A } & 119.62(11) \\ \text { C6A-C5A-C11 } & 121.80(10) \\ \text { C4A-C5A-C11 } & 118.58(10) \\ \text { N1A-C6A-C5A } & 120.87(11) \\ \text { N1A-C6A-C8A } & 116.99(12) \\ \text { C5A-C6A-C8A } & 122.14(12) \\ \text { C2A-C7A-H7AA } & 109.5 \\ \text { C2A-C7A-H7AB } & 109.5 \\ \text { H7AA-C7A-H7AB } & 109.5 \\ \text { C2A-C7A-H7AC } & 109.5 \\ \text { H7AA-C7A-H7AC } & 109.5 \\ \text { H7AB-C7A-H7AC } & 109.5 \\ \text { C6A-C8A-H8AA } & 109.5 \\ \text { C6A-C8A-H8AB } & 109.5 \\ \text { H8AA-C8A-H8AB } & 109.5 \\ \text { C6A-C8A-H8AC } & 109.5 \\ \text { H8AA-C8A-H8AC } & 109.5 \\ \text { H8AB-C8A-H8AC } & 109.5 \\ & \\ \text { C6A-N1A-C2A-N3A } & 0.22(19) \\ \text { C6A-N1A-C2A-C7A } & -179.47(11) \\ \text { N1A-C2A-N3A-C4A } & -0.97(19) \\ \text { C7A-C2A-N3A-C4A } & 178.73(11) \\ \text { C2A-N3A-C4A-N4A } & -179.68(12) \\ \text { C2A-N3A-C4A-C5A } & 0.64(18) \\ \text { N4A-C4A-C5A-C6A } & -179.33(12) \\ \text { N3A-C4A-C5A-C6A } & 0.33(18) \\ \text { N4A-C4A-C5A-C11 } & 0.54(18) \\ \text { N3A-C4A-C5A-C11 } & -179.80(9) \\ \text { C2A-N1A-C6A-C5A } & 0.82(18) \\ \text { C2A-N1A-C6A-C8A } & -178.96(11) \\ \text { C4A-C5A-C6A-N1A } & -1.09(19) \\ \text { C11-C5A-C6A-N1A } & 179.04(10) \\ \text { C4A-C5A-C6A-C8A } & 178.68(12) \\ & \end{array}$

$\mathrm{C} 5 \mathrm{~B}-\mathrm{C} 4 \mathrm{~B}-\mathrm{H} 4 \mathrm{BA}$

$\mathrm{C} 3 \mathrm{~B}-\mathrm{C} 4 \mathrm{~B}-\mathrm{H} 4 \mathrm{BA}$

$\mathrm{C} 6 \mathrm{~B}-\mathrm{C} 5 \mathrm{~B}-\mathrm{C} 4 \mathrm{~B}$

$\mathrm{C} 6 \mathrm{~B}-\mathrm{C} 5 \mathrm{~B}-\mathrm{H} 5 \mathrm{BA}$

$\mathrm{C} 4 \mathrm{~B}-\mathrm{C} 5 \mathrm{~B}-\mathrm{H} 5 \mathrm{BA}$

$\mathrm{C} 5 \mathrm{~B}-\mathrm{C} 6 \mathrm{~B}-\mathrm{C} 1 \mathrm{~B}$

$\mathrm{C} 5 \mathrm{~B}-\mathrm{C} 6 \mathrm{~B}-\mathrm{H} 6 \mathrm{BA}$

$\mathrm{C} 1 \mathrm{~B}-\mathrm{C} 6 \mathrm{~B}-\mathrm{H} 6 \mathrm{BA}$

$\mathrm{O} 2 \mathrm{~B}-\mathrm{C} 7 \mathrm{~B}-\mathrm{O} 1 \mathrm{~B}$

$\mathrm{O} 2 \mathrm{~B}-\mathrm{C} 7 \mathrm{~B}-\mathrm{C} 1 \mathrm{~B}$

$\mathrm{O} 1 \mathrm{~B}-\mathrm{C} 7 \mathrm{~B}-\mathrm{C} 1 \mathrm{~B}$

$\mathrm{C} 3 \mathrm{~B}-\mathrm{C} 8 \mathrm{~B}-\mathrm{H} 8 \mathrm{BA}$

$\mathrm{C} 3 \mathrm{~B}-\mathrm{C} 8 \mathrm{~B}-\mathrm{H} 8 \mathrm{BB}$

$\mathrm{H} 8 \mathrm{BA}-\mathrm{C} 8 \mathrm{~B}-\mathrm{H} 8 \mathrm{BB}$

$\mathrm{C} 3 \mathrm{~B}-\mathrm{C} 8 \mathrm{~B}-\mathrm{H} 8 \mathrm{BC}$

H8BA-C $8 B-\mathrm{H} 8 \mathrm{BC}$

$\mathrm{H} 8 \mathrm{BB}-\mathrm{C} 8 \mathrm{~B}-\mathrm{H} 8 \mathrm{BC}$

$\mathrm{C} 11-\mathrm{C} 5 \mathrm{~A}-\mathrm{C} 6 \mathrm{~A}-\mathrm{C} 8 \mathrm{~A}$
$\mathrm{C} 6 \mathrm{~B}-\mathrm{C} 1 \mathrm{~B}-\mathrm{C} 2 \mathrm{~B}-\mathrm{C} 3 \mathrm{~B}$
$\mathrm{C} 7 \mathrm{~B}-\mathrm{C} 1 \mathrm{~B}-\mathrm{C} 2 \mathrm{~B}-\mathrm{C} 3 \mathrm{~B}$
$\mathrm{C} 1 \mathrm{~B}-\mathrm{C} 2 \mathrm{~B}-\mathrm{C} 3 \mathrm{~B}-\mathrm{C} 4 \mathrm{~B}$
$\mathrm{C} 1 \mathrm{~B}-\mathrm{C} 2 \mathrm{~B}-\mathrm{C} 3 \mathrm{~B}-\mathrm{C} 8 \mathrm{~B}$
$\mathrm{C} 2 \mathrm{~B}-\mathrm{C} 3 \mathrm{~B}-\mathrm{C} 4 \mathrm{~B}-\mathrm{C} 5 \mathrm{~B}$
$\mathrm{C} 8 \mathrm{~B}-\mathrm{C} 3 \mathrm{~B}-\mathrm{C} 4 \mathrm{~B}-\mathrm{C} 5 \mathrm{~B}$
$\mathrm{C} 3 \mathrm{~B}-\mathrm{C} 4 \mathrm{~B}-\mathrm{C} 5 \mathrm{~B}-\mathrm{C} 6 \mathrm{~B}$
$\mathrm{C} 4 \mathrm{~B}-\mathrm{C} 5 \mathrm{~B}-\mathrm{C} 6 \mathrm{~B}-\mathrm{C} 1 \mathrm{~B}$
$\mathrm{C} 2 \mathrm{~B}-\mathrm{C} 1 \mathrm{~B}-\mathrm{C} 6 \mathrm{~B}-\mathrm{C} 5 \mathrm{~B}$
$\mathrm{C} 7 \mathrm{~B}-\mathrm{C} 1 \mathrm{~B}-\mathrm{C} 6 \mathrm{~B}-\mathrm{C} 5 \mathrm{~B}$
$\mathrm{C} 6 \mathrm{~B}-\mathrm{C} 1 \mathrm{~B}-\mathrm{C} 7 \mathrm{~B}-\mathrm{O} 2 \mathrm{~B}$
$\mathrm{C} 2 \mathrm{~B}-\mathrm{C} 1 \mathrm{~B}-\mathrm{C} 7 \mathrm{~B}-\mathrm{O} 2 \mathrm{~B}$
$\mathrm{C} 6 \mathrm{~B}-\mathrm{C} 1 \mathrm{~B}-\mathrm{C} 7 \mathrm{~B}-\mathrm{O} 1 \mathrm{~B}$
$\mathrm{C} 2 \mathrm{~B}-\mathrm{C} 1 \mathrm{~B}-\mathrm{C} 7 \mathrm{~B}-\mathrm{O} 1 \mathrm{~B}$
119.4

119.4

120.20 (14)

119.9

119.9

$119.30(13)$

120.3

120.3

$123.72(12)$

$121.95(12)$

$114.33(11)$

109.5

109.5

109.5

109.5

109.5

109.5

$$
\begin{aligned}
& -1.18(18) \\
& -0.8(2) \\
& 178.57(12) \\
& 0.9(2) \\
& -179.30(13) \\
& -0.2(2) \\
& -179.97(14) \\
& -0.7(2) \\
& 0.8(2) \\
& -0.1(2) \\
& -179.46(13) \\
& 12.8(2) \\
& -166.53(12) \\
& -167.53(12) \\
& 13.12(18)
\end{aligned}
$$

$13.12(18)$

Hydrogen-bond geometry $\left(A,{ }^{\circ}\right)$

\begin{tabular}{lllll}
\hline$D-\mathrm{H} \cdots A$ & $D-\mathrm{H}$ & $\mathrm{H} \cdots A$ & $D \cdots A$ & $D-\mathrm{H} \cdots A$ \\
\hline $\mathrm{N} 4 A-\mathrm{H} 41 \cdots \mathrm{O} 2 B$ & $0.829(18)$ & $2.055(19)$ & $2.8802(15)$ & $174.2(17)$ \\
$\mathrm{N} 4 A-\mathrm{H} 42 \cdots \mathrm{N} 1 A^{\mathrm{i}}$ & $0.90(2)$ & $2.23(2)$ & $3.0332(16)$ & $149.0(17)$ \\
$\mathrm{C} 7 A-\mathrm{H} 7 A A \cdots \mathrm{C} 11^{\mathrm{ii}}$ & 0.98 & 2.97 & $3.5919(14)$ & 123 \\
$\mathrm{C} 7 A-\mathrm{H} 7 A A \cdots \mathrm{N} 4 A^{\mathrm{iii}}$ & 0.98 & 2.67 & $3.4667(18)$ & 139 \\
$\mathrm{C} 8 A-\mathrm{H} 8 A A \cdots \mathrm{O} 2 B^{\mathrm{iv}}$ & 0.98 & 2.65 & $3.3295(17)$ & 127 \\
$\mathrm{O} 1 B-\mathrm{H} 1 B \cdots \mathrm{N} 3 A$ & $0.88(2)$ & $1.79(2)$ & $2.6701(15)$ & $173(2)$ \\
\hline
\end{tabular}

Symmetry codes: (i) $-x+1 / 2, y-1 / 2, z$; (ii) $x+1 / 2, y+1 / 2,-z+1 / 2$; (iii) $-x+1 / 2, y+1 / 2, z$; (iv) $x-1 / 2, y+1 / 2,-z+1 / 2$. 
4-Amino-5-chloro-2,6-dimethylpyrimidine-4-methylbenzoic acid (1/1) (COCRYSTAL-9)

Crystal data

$\mathrm{C}_{6} \mathrm{H}_{8} \mathrm{ClN}_{3} \cdot \mathrm{C}_{8} \mathrm{H}_{8} \mathrm{O}_{2}$

$M_{r}=293.75$

Monoclinic, $P 2_{1} / c$

$a=8.0223(5) \AA$

$b=13.6901(10) \AA$

$c=13.3346(10) \AA$

$\beta=101.557(7)^{\circ}$

$V=1434.80(18) \AA^{3}$

$Z=4$

\section{Data collection}

Agilent SuperNova Dual Source diffractometer with an Atlas detector

Radiation source: sealed X-ray tube Detector resolution: 10.6501 pixels $\mathrm{mm}^{-1}$ $\omega$ scans

Absorption correction: multi-scan

(CrysAlis PRO; Agilent, 2014)

$T_{\min }=0.582, T_{\max }=1.000$

\section{Refinement}

Refinement on $F^{2}$

Least-squares matrix: full

$R\left[F^{2}>2 \sigma\left(F^{2}\right)\right]=0.047$

$w R\left(F^{2}\right)=0.140$

$S=1.04$

2966 reflections

197 parameters

0 restraints

Hydrogen site location: mixed
$F(000)=616$

$D_{\mathrm{x}}=1.360 \mathrm{Mg} \mathrm{m}^{-3}$

$\mathrm{Cu} K \alpha$ radiation, $\lambda=1.54184 \AA$

Cell parameters from 2368 reflections

$\theta=4.7-76.5^{\circ}$

$\mu=2.41 \mathrm{~mm}^{-1}$

$T=120 \mathrm{~K}$

Plate, colorless

$0.51 \times 0.17 \times 0.08 \mathrm{~mm}$

6765 measured reflections

2966 independent reflections

2422 reflections with $I>2 \sigma(I)$

$R_{\text {int }}=0.040$

$\theta_{\max }=76.7^{\circ}, \theta_{\min }=4.7^{\circ}$

$h=-8 \rightarrow 10$

$k=-17 \rightarrow 17$

$l=-16 \rightarrow 15$

$\mathrm{H}$ atoms treated by a mixture of independent and constrained refinement

$w=1 /\left[\sigma^{2}\left(F_{\mathrm{o}}^{2}\right)+(0.0813 P)^{2}+0.4428 P\right]$

where $P=\left(F_{\mathrm{o}}^{2}+2 F_{\mathrm{c}}^{2}\right) / 3$

$(\Delta / \sigma)_{\max }=0.001$

$\Delta \rho_{\max }=0.28$ e $\AA^{-3}$

$\Delta \rho_{\text {min }}=-0.41$ e $\AA^{-3}$

Extinction correction: SHELXL2014

(Sheldrick, 2015b),

$\mathrm{Fc}^{*}=\mathrm{kFc}\left[1+0.001 \times \mathrm{Fc}^{2} \lambda^{3} / \sin (2 \theta)\right]^{-1 / 4}$

Extinction coefficient: 0.0014 (4)

\section{Special details}

Geometry. All esds (except the esd in the dihedral angle between two 1.s. planes) are estimated using the full covariance matrix. The cell esds are taken into account individually in the estimation of esds in distances, angles and torsion angles; correlations between esds in cell parameters are only used when they are defined by crystal symmetry. An approximate (isotropic) treatment of cell esds is used for estimating esds involving l.s. planes.

Fractional atomic coordinates and isotropic or equivalent isotropic displacement parameters $\left(\AA^{2}\right)$

\begin{tabular}{lllll}
\hline & $x$ & $y$ & $z$ & $U_{\text {iso }} * / U_{\text {eq }}$ \\
\hline C11 & $1.08463(7)$ & $0.85968(4)$ & $0.61237(4)$ & $0.03229(19)$ \\
N1A & $0.9592(2)$ & $0.72109(13)$ & $0.34491(13)$ & $0.0271(4)$ \\
C2A & $0.8839(3)$ & $0.64300(15)$ & $0.37583(16)$ & $0.0264(4)$ \\
N3A & $0.8637(2)$ & $0.62518(13)$ & $0.47148(13)$ & $0.0262(4)$ \\
N4A & $0.8970(2)$ & $0.67557(14)$ & $0.63951(14)$ & $0.0297(4)$ \\
H41 & $0.852(3)$ & $0.625(2)$ & $0.647(2)$ & $0.030(7)^{*}$ \\
H42 & $0.934(3)$ & $0.719(2)$ & $0.691(2)$ & $0.042(7)^{*}$ \\
C4A & $0.9222(2)$ & $0.69202(15)$ & $0.54527(15)$ & $0.0253(4)$
\end{tabular}




$\begin{array}{lllll}\text { C5A } & 1.0068(2) & 0.77534(15) & 0.51821(15) & 0.0265(4) \\ \text { C6A } & 1.0238(2) & 0.78780(15) & 0.41779(15) & 0.0259(4) \\ \text { C7A } & 0.8182(3) & 0.56630(16) & 0.29745(16) & 0.0323(5) \\ \text { H7AA } & 0.8067 & 0.5941 & 0.2287 & 0.048^{*} \\ \text { H7AB } & 0.7069 & 0.5434 & 0.3073 & 0.048^{*} \\ \text { H7AC } & 0.8980 & 0.5113 & 0.3051 & 0.048^{*} \\ \text { C8A } & 1.1093(3) & 0.87516(16) & 0.38284(17) & 0.0308(4) \\ \text { H8AA } & 1.1603 & 0.8570 & 0.3246 & 0.046^{*} \\ \text { H8AB } & 1.1984 & 0.8986 & 0.4390 & 0.046^{*} \\ \text { H8AC } & 1.0252 & 0.9270 & 0.3621 & 0.046^{*} \\ \text { O1B } & 0.7077(2) & 0.45998(12) & 0.49384(12) & 0.0375(4) \\ \text { H1B } & 0.757(5) & 0.519(3) & 0.492(3) & 0.089(13)^{*} \\ \text { O2B } & 0.7255(2) & 0.48776(12) & 0.66154(12) & 0.0373(4) \\ \text { C1B } & 0.5926(3) & 0.34164(16) & 0.58705(17) & 0.0288(4) \\ \text { C2B } & 0.5582(3) & 0.30639(17) & 0.67918(17) & 0.0310(5) \\ \text { H2BA } & 0.5902 & 0.3436 & 0.7401 & 0.037^{*} \\ \text { C3B } & 0.4773(3) & 0.21712(17) & 0.68174(17) & 0.0321(5) \\ \text { H3BA } & 0.4540 & 0.1940 & 0.7447 & 0.038^{*} \\ \text { C4B } & 0.4296(3) & 0.16065(16) & 0.59342(17) & 0.0304(5) \\ \text { C5B } & 0.4629(3) & 0.19749(17) & 0.50191(17) & 0.0328(5) \\ \text { H5BA } & 0.4295 & 0.1608 & 0.4407 & 0.039^{*} \\ \text { C6B } & 0.5437(3) & 0.28656(16) & 0.49835(16) & 0.0304(5) \\ \text { H6BA } & 0.5658 & 0.3100 & 0.4352 & 0.037^{*} \\ \text { C7B } & 0.6814(3) & 0.43686(16) & 0.58525(16) & 0.0298(4) \\ \text { C8B } & 0.3480(3) & 0.06233(18) & 0.59702(19) & 0.0384(5) \\ \text { H8BA } & 0.2421 & 0.0597 & 0.5456 & 0.058^{*} \\ \text { H8BB } & 0.3230 & 0.0518 & 0.6652 & 0.058^{*} \\ \text { H8BC } & 0.4258 & 0.0114 & 0.5826 & 0.058^{*} \\ & & & & \end{array}$

Atomic displacement parameters $\left(\AA^{2}\right)$

\begin{tabular}{lllllll}
\hline & $U^{11}$ & $U^{22}$ & $U^{33}$ & $U^{12}$ & $U^{13}$ & $U^{23}$ \\
\hline C11 & $0.0424(3)$ & $0.0267(3)$ & $0.0290(3)$ & $-0.00801(19)$ & $0.0100(2)$ & $-0.00421(18)$ \\
N1A & $0.0310(8)$ & $0.0251(9)$ & $0.0267(8)$ & $-0.0005(7)$ & $0.0094(6)$ & $-0.0003(7)$ \\
C2A & $0.0289(9)$ & $0.0233(10)$ & $0.0279(10)$ & $0.0017(7)$ & $0.0083(8)$ & $-0.0017(8)$ \\
N3A & $0.0303(8)$ & $0.0220(8)$ & $0.0274(8)$ & $-0.0006(7)$ & $0.0080(6)$ & $0.0003(7)$ \\
N4A & $0.0422(10)$ & $0.0216(9)$ & $0.0263(9)$ & $-0.0077(8)$ & $0.0096(7)$ & $-0.0003(7)$ \\
C4A & $0.0281(9)$ & $0.0222(9)$ & $0.0262(9)$ & $0.0017(7)$ & $0.0068(7)$ & $0.0010(8)$ \\
C5A & $0.0282(9)$ & $0.0226(10)$ & $0.0298(10)$ & $0.0008(8)$ & $0.0081(8)$ & $-0.0012(8)$ \\
C6A & $0.0257(9)$ & $0.0225(10)$ & $0.0307(10)$ & $0.0021(7)$ & $0.0086(7)$ & $0.0019(8)$ \\
C7A & $0.0425(11)$ & $0.0280(11)$ & $0.0286(10)$ & $-0.0036(9)$ & $0.0124(8)$ & $-0.0041(9)$ \\
C8A & $0.0351(10)$ & $0.0279(10)$ & $0.0312(10)$ & $-0.0025(9)$ & $0.0112(8)$ & $0.0020(8)$ \\
O1B & $0.0513(10)$ & $0.0307(8)$ & $0.0339(8)$ & $-0.0130(7)$ & $0.0165(7)$ & $-0.0017(7)$ \\
O2B & $0.0485(9)$ & $0.0316(8)$ & $0.0331(8)$ & $-0.0099(7)$ & $0.0113(7)$ & $-0.0036(7)$ \\
C1B & $0.0282(10)$ & $0.0251(10)$ & $0.0340(10)$ & $-0.0005(8)$ & $0.0088(8)$ & $0.0000(8)$ \\
C2B & $0.0321(10)$ & $0.0306(11)$ & $0.0309(10)$ & $-0.0025(9)$ & $0.0076(8)$ & $-0.0012(9)$ \\
C3B & $0.0330(10)$ & $0.0334(12)$ & $0.0315(10)$ & $-0.0023(9)$ & $0.0105(8)$ & $0.0042(9)$ \\
C4B & $0.0272(10)$ & $0.0266(10)$ & $0.0383(11)$ & $0.0003(8)$ & $0.0091(8)$ & $0.0008(9)$
\end{tabular}




\begin{tabular}{lllllll} 
C5B & $0.0359(11)$ & $0.0276(11)$ & $0.0352(11)$ & $-0.0023(9)$ & $0.0081(9)$ & $-0.0044(9)$ \\
C6B & $0.0341(10)$ & $0.0287(11)$ & $0.0295(10)$ & $-0.0016(8)$ & $0.0087(8)$ & $0.0009(8)$ \\
C7B & $0.0305(10)$ & $0.0274(10)$ & $0.0329(10)$ & $0.0000(8)$ & $0.0092(8)$ & $0.0005(9)$ \\
C8B & $0.0407(12)$ & $0.0297(12)$ & $0.0470(13)$ & $-0.0035(9)$ & $0.0138(10)$ & $0.0027(10)$ \\
\hline
\end{tabular}

Geometric parameters $\left(\hat{A},{ }^{\circ}\right)$

\begin{tabular}{|c|c|c|c|}
\hline $\mathrm{C} 11-\mathrm{C} 5 \mathrm{~A}$ & $1.728(2)$ & $\mathrm{O} 1 \mathrm{~B}-\mathrm{C} 7 \mathrm{~B}$ & $1.317(3)$ \\
\hline $\mathrm{N} 1 \mathrm{~A}-\mathrm{C} 2 \mathrm{~A}$ & $1.333(3)$ & $\mathrm{O} 1 \mathrm{~B}-\mathrm{H} 1 \mathrm{~B}$ & $0.90(4)$ \\
\hline $\mathrm{N} 1 \mathrm{~A}-\mathrm{C} 6 \mathrm{~A}$ & $1.358(3)$ & $\mathrm{O} 2 \mathrm{~B}-\mathrm{C} 7 \mathrm{~B}$ & $1.225(3)$ \\
\hline $\mathrm{C} 2 \mathrm{~A}-\mathrm{N} 3 \mathrm{~A}$ & $1.340(3)$ & $\mathrm{C} 1 \mathrm{~B}-\mathrm{C} 6 \mathrm{~B}$ & $1.391(3)$ \\
\hline $\mathrm{C} 2 \mathrm{~A}-\mathrm{C} 7 \mathrm{~A}$ & $1.501(3)$ & $\mathrm{C} 1 \mathrm{~B}-\mathrm{C} 2 \mathrm{~B}$ & $1.398(3)$ \\
\hline $\mathrm{N} 3 \mathrm{~A}-\mathrm{C} 4 \mathrm{~A}$ & $1.357(3)$ & $\mathrm{C} 1 \mathrm{~B}-\mathrm{C} 7 \mathrm{~B}$ & $1.488(3)$ \\
\hline $\mathrm{N} 4 \mathrm{~A}-\mathrm{C} 4 \mathrm{~A}$ & $1.331(3)$ & $\mathrm{C} 2 \mathrm{~B}-\mathrm{C} 3 \mathrm{~B}$ & $1.387(3)$ \\
\hline $\mathrm{N} 4 \mathrm{~A}-\mathrm{H} 41$ & $0.79(3)$ & $\mathrm{C} 2 \mathrm{~B}-\mathrm{H} 2 \mathrm{BA}$ & 0.9500 \\
\hline $\mathrm{N} 4 \mathrm{~A}-\mathrm{H} 42$ & $0.91(3)$ & $\mathrm{C} 3 \mathrm{~B}-\mathrm{C} 4 \mathrm{~B}$ & $1.397(3)$ \\
\hline $\mathrm{C} 4 \mathrm{~A}-\mathrm{C} 5 \mathrm{~A}$ & $1.410(3)$ & $\mathrm{C} 3 \mathrm{~B}-\mathrm{H} 3 \mathrm{BA}$ & 0.9500 \\
\hline $\mathrm{C} 5 \mathrm{~A}-\mathrm{C} 6 \mathrm{~A}$ & $1.383(3)$ & $\mathrm{C} 4 \mathrm{~B}-\mathrm{C} 5 \mathrm{~B}$ & $1.395(3)$ \\
\hline $\mathrm{C} 6 \mathrm{~A}-\mathrm{C} 8 \mathrm{~A}$ & $1.499(3)$ & $\mathrm{C} 4 \mathrm{~B}-\mathrm{C} 8 \mathrm{~B}$ & $1.502(3)$ \\
\hline C7A-H7AA & 0.9800 & $\mathrm{C} 5 \mathrm{~B}-\mathrm{C} 6 \mathrm{~B}$ & $1.386(3)$ \\
\hline $\mathrm{C} 7 \mathrm{~A}-\mathrm{H} 7 \mathrm{AB}$ & 0.9800 & $\mathrm{C} 5 \mathrm{~B}-\mathrm{H} 5 \mathrm{BA}$ & 0.9500 \\
\hline $\mathrm{C} 7 \mathrm{~A}-\mathrm{H} 7 \mathrm{AC}$ & 0.9800 & $\mathrm{C} 6 \mathrm{~B}-\mathrm{H} 6 \mathrm{BA}$ & 0.9500 \\
\hline $\mathrm{C} 8 \mathrm{~A}-\mathrm{H} 8 \mathrm{AA}$ & 0.9800 & $\mathrm{C} 8 \mathrm{~B}-\mathrm{H} 8 \mathrm{BA}$ & 0.9800 \\
\hline $\mathrm{C} 8 \mathrm{~A}-\mathrm{H} 8 \mathrm{AB}$ & 0.9800 & $\mathrm{C} 8 \mathrm{~B}-\mathrm{H} 8 \mathrm{BB}$ & 0.9800 \\
\hline $\mathrm{C} 8 \mathrm{~A}-\mathrm{H} 8 \mathrm{AC}$ & 0.9800 & $\mathrm{C} 8 \mathrm{~B}-\mathrm{H} 8 \mathrm{BC}$ & 0.9800 \\
\hline $\mathrm{C} 2 \mathrm{~A}-\mathrm{N} 1 \mathrm{~A}-\mathrm{C} 6 \mathrm{~A}$ & $116.70(17)$ & $\mathrm{C} 7 \mathrm{~B}-\mathrm{O} 1 \mathrm{~B}-\mathrm{H} 1 \mathrm{~B}$ & $113(3)$ \\
\hline $\mathrm{N} 1 \mathrm{~A}-\mathrm{C} 2 \mathrm{~A}-\mathrm{N} 3 \mathrm{~A}$ & $126.09(19)$ & $\mathrm{C} 6 \mathrm{~B}-\mathrm{C} 1 \mathrm{~B}-\mathrm{C} 2 \mathrm{~B}$ & $119.2(2)$ \\
\hline $\mathrm{N} 1 \mathrm{~A}-\mathrm{C} 2 \mathrm{~A}-\mathrm{C} 7 \mathrm{~A}$ & $117.61(18)$ & $\mathrm{C} 6 \mathrm{~B}-\mathrm{C} 1 \mathrm{~B}-\mathrm{C} 7 \mathrm{~B}$ & $121.0(2)$ \\
\hline $\mathrm{N} 3 \mathrm{~A}-\mathrm{C} 2 \mathrm{~A}-\mathrm{C} 7 \mathrm{~A}$ & $116.28(18)$ & $\mathrm{C} 2 \mathrm{~B}-\mathrm{C} 1 \mathrm{~B}-\mathrm{C} 7 \mathrm{~B}$ & $119.7(2)$ \\
\hline $\mathrm{C} 2 \mathrm{~A}-\mathrm{N} 3 \mathrm{~A}-\mathrm{C} 4 \mathrm{~A}$ & $118.50(18)$ & $\mathrm{C} 3 \mathrm{~B}-\mathrm{C} 2 \mathrm{~B}-\mathrm{C} 1 \mathrm{~B}$ & $120.1(2)$ \\
\hline $\mathrm{C} 4 \mathrm{~A}-\mathrm{N} 4 \mathrm{~A}-\mathrm{H} 41$ & $114.9(19)$ & $\mathrm{C} 3 \mathrm{~B}-\mathrm{C} 2 \mathrm{~B}-\mathrm{H} 2 \mathrm{BA}$ & 120.0 \\
\hline $\mathrm{C} 4 \mathrm{~A}-\mathrm{N} 4 \mathrm{~A}-\mathrm{H} 42$ & $121.2(18)$ & $\mathrm{C} 1 \mathrm{~B}-\mathrm{C} 2 \mathrm{~B}-\mathrm{H} 2 \mathrm{BA}$ & 120.0 \\
\hline $\mathrm{H} 41-\mathrm{N} 4 \mathrm{~A}-\mathrm{H} 42$ & $124(3)$ & $\mathrm{C} 2 \mathrm{~B}-\mathrm{C} 3 \mathrm{~B}-\mathrm{C} 4 \mathrm{~B}$ & $121.2(2)$ \\
\hline $\mathrm{N} 4 \mathrm{~A}-\mathrm{C} 4 \mathrm{~A}-\mathrm{N} 3 \mathrm{~A}$ & $118.32(19)$ & $\mathrm{C} 2 \mathrm{~B}-\mathrm{C} 3 \mathrm{~B}-\mathrm{H} 3 \mathrm{BA}$ & 119.4 \\
\hline $\mathrm{N} 4 \mathrm{~A}-\mathrm{C} 4 \mathrm{~A}-\mathrm{C} 5 \mathrm{~A}$ & $123.37(19)$ & $\mathrm{C} 4 \mathrm{~B}-\mathrm{C} 3 \mathrm{~B}-\mathrm{H} 3 \mathrm{BA}$ & 119.4 \\
\hline $\mathrm{N} 3 \mathrm{~A}-\mathrm{C} 4 \mathrm{~A}-\mathrm{C} 5 \mathrm{~A}$ & $118.31(18)$ & $\mathrm{C} 5 \mathrm{~B}-\mathrm{C} 4 \mathrm{~B}-\mathrm{C} 3 \mathrm{~B}$ & $118.0(2)$ \\
\hline $\mathrm{C} 6 \mathrm{~A}-\mathrm{C} 5 \mathrm{~A}-\mathrm{C} 4 \mathrm{~A}$ & $119.55(19)$ & $\mathrm{C} 5 \mathrm{~B}-\mathrm{C} 4 \mathrm{~B}-\mathrm{C} 8 \mathrm{~B}$ & $120.9(2)$ \\
\hline $\mathrm{C} 6 \mathrm{~A}-\mathrm{C} 5 \mathrm{~A}-\mathrm{C} 11$ & $121.97(16)$ & $\mathrm{C} 3 \mathrm{~B}-\mathrm{C} 4 \mathrm{~B}-\mathrm{C} 8 \mathrm{~B}$ & $121.1(2)$ \\
\hline $\mathrm{C} 4 \mathrm{~A}-\mathrm{C} 5 \mathrm{~A}-\mathrm{C} 11$ & $118.48(15)$ & $\mathrm{C} 6 \mathrm{~B}-\mathrm{C} 5 \mathrm{~B}-\mathrm{C} 4 \mathrm{~B}$ & $121.4(2)$ \\
\hline $\mathrm{N} 1 \mathrm{~A}-\mathrm{C} 6 \mathrm{~A}-\mathrm{C} 5 \mathrm{~A}$ & $120.80(18)$ & $\mathrm{C} 6 \mathrm{~B}-\mathrm{C} 5 \mathrm{~B}-\mathrm{H} 5 \mathrm{BA}$ & 119.3 \\
\hline $\mathrm{N} 1 \mathrm{~A}-\mathrm{C} 6 \mathrm{~A}-\mathrm{C} 8 \mathrm{~A}$ & $116.54(18)$ & $\mathrm{C} 4 \mathrm{~B}-\mathrm{C} 5 \mathrm{~B}-\mathrm{H} 5 \mathrm{BA}$ & 119.3 \\
\hline $\mathrm{C} 5 \mathrm{~A}-\mathrm{C} 6 \mathrm{~A}-\mathrm{C} 8 \mathrm{~A}$ & $122.64(19)$ & $\mathrm{C} 5 \mathrm{~B}-\mathrm{C} 6 \mathrm{~B}-\mathrm{C} 1 \mathrm{~B}$ & $120.1(2)$ \\
\hline $\mathrm{C} 2 \mathrm{~A}-\mathrm{C} 7 \mathrm{~A}-\mathrm{H} 7 \mathrm{AA}$ & 109.5 & $\mathrm{C} 5 \mathrm{~B}-\mathrm{C} 6 \mathrm{~B}-\mathrm{H} 6 \mathrm{BA}$ & 119.9 \\
\hline $\mathrm{C} 2 \mathrm{~A}-\mathrm{C} 7 \mathrm{~A}-\mathrm{H} 7 \mathrm{AB}$ & 109.5 & $\mathrm{C} 1 \mathrm{~B}-\mathrm{C} 6 \mathrm{~B}-\mathrm{H} 6 \mathrm{BA}$ & 119.9 \\
\hline $\mathrm{H} 7 \mathrm{AA}-\mathrm{C} 7 \mathrm{~A}-\mathrm{H} 7 \mathrm{AB}$ & 109.5 & $\mathrm{O} 2 \mathrm{~B}-\mathrm{C} 7 \mathrm{~B}-\mathrm{O} 1 \mathrm{~B}$ & $124.0(2)$ \\
\hline $\mathrm{C} 2 \mathrm{~A}-\mathrm{C} 7 \mathrm{~A}-\mathrm{H} 7 \mathrm{AC}$ & 109.5 & $\mathrm{O} 2 \mathrm{~B}-\mathrm{C} 7 \mathrm{~B}-\mathrm{C} 1 \mathrm{~B}$ & $123.0(2)$ \\
\hline H7AA-C7A-H7AC & 109.5 & $\mathrm{O} 1 \mathrm{~B}-\mathrm{C} 7 \mathrm{~B}-\mathrm{C} 1 \mathrm{~B}$ & $113.06(19)$ \\
\hline
\end{tabular}




$\begin{array}{ll}\mathrm{H} 7 \mathrm{AB}-\mathrm{C} 7 \mathrm{~A}-\mathrm{H} 7 \mathrm{AC} & 109.5 \\ \mathrm{C} 6 \mathrm{~A}-\mathrm{C} 8 \mathrm{~A}-\mathrm{H} 8 \mathrm{AA} & 109.5 \\ \mathrm{C} 6 \mathrm{~A}-\mathrm{C} 8 \mathrm{~A}-\mathrm{H} 8 \mathrm{AB} & 109.5 \\ \mathrm{H} 8 \mathrm{AA}-\mathrm{C} 8 \mathrm{~A}-\mathrm{H} 8 \mathrm{AB} & 109.5 \\ \mathrm{C} 6 \mathrm{~A}-\mathrm{C} 8 \mathrm{~A}-\mathrm{H} 8 \mathrm{AC} & 109.5 \\ \mathrm{H} 8 \mathrm{AA}-\mathrm{C} 8 \mathrm{~A}-\mathrm{H} 8 \mathrm{AC} & 109.5 \\ \mathrm{H} 8 \mathrm{AB}-\mathrm{C} 8 \mathrm{~A}-\mathrm{H} 8 \mathrm{AC} & 109.5 \\ & \\ \mathrm{C} 6 \mathrm{~A}-\mathrm{N} 1 \mathrm{~A}-\mathrm{C} 2 \mathrm{~A}-\mathrm{N} 3 \mathrm{~A} & 1.2(3) \\ \mathrm{C} 6 \mathrm{~A}-\mathrm{N} 1 \mathrm{~A}-\mathrm{C} 2 \mathrm{~A}-\mathrm{C} 7 \mathrm{~A} & -177.48(18) \\ \mathrm{N} 1 \mathrm{~A}-\mathrm{C} 2 \mathrm{~A}-\mathrm{N} 3 \mathrm{~A}-\mathrm{C} 4 \mathrm{~A} & 0.8(3) \\ \mathrm{C} 7 \mathrm{~A}-\mathrm{C} 2 \mathrm{~A}-\mathrm{N} 3 \mathrm{~A}-\mathrm{C} 4 \mathrm{~A} & 179.52(18) \\ \mathrm{C} 2 \mathrm{~A}-\mathrm{N} 3 \mathrm{~A}-\mathrm{C} 4 \mathrm{~A}-\mathrm{N} 4 \mathrm{~A} & 177.86(18) \\ \mathrm{C} 2 \mathrm{~A}-\mathrm{N} 3 \mathrm{~A}-\mathrm{C} 4 \mathrm{~A}-\mathrm{C} 5 \mathrm{~A} & -2.2(3) \\ \mathrm{N} 4 \mathrm{~A}-\mathrm{C} 4 \mathrm{~A}-\mathrm{C} 5 \mathrm{~A}-\mathrm{C} 6 \mathrm{~A} & -178.43(19) \\ \mathrm{N} 3 \mathrm{~A}-\mathrm{C} 4 \mathrm{~A}-\mathrm{C} 5 \mathrm{~A}-\mathrm{C} 6 \mathrm{~A} & 1.6(3) \\ \mathrm{N} 4 \mathrm{~A}-\mathrm{C} 4 \mathrm{~A}-\mathrm{C} 5 \mathrm{~A}-\mathrm{C} 11 & 1.0(3) \\ \mathrm{N} 3 \mathrm{~A}-\mathrm{C} 4 \mathrm{~A}-\mathrm{C} 5 \mathrm{~A}-\mathrm{C} 11 & -178.94(14) \\ \mathrm{C} 2 \mathrm{~A}-\mathrm{N} 1 \mathrm{~A}-\mathrm{C} 6 \mathrm{~A}-\mathrm{C} 5 \mathrm{~A} & -1.8(3) \\ \mathrm{C} 2 \mathrm{~A}-\mathrm{N} 1 \mathrm{~A}-\mathrm{C} 6 \mathrm{~A}-\mathrm{C} 8 \mathrm{~A} & 179.60(17) \\ \mathrm{C} 4 \mathrm{~A}-\mathrm{C} 5 \mathrm{~A}-\mathrm{C} 6 \mathrm{~A}-\mathrm{N} 1 \mathrm{~A} & 0.4(3) \\ \mathrm{C} 11-\mathrm{C} 5 \mathrm{~A}-\mathrm{C} 6 \mathrm{~A}-\mathrm{N} 1 \mathrm{~A} & -179.01(15) \\ \mathrm{C} 4 \mathrm{~A}-\mathrm{C} 5 \mathrm{~A}-\mathrm{C} 6 \mathrm{~A}-\mathrm{C} 8 \mathrm{~A} & 178.96(18) \\ \end{array}$

$\mathrm{C} 4 \mathrm{~B}-\mathrm{C} 8 \mathrm{~B}-\mathrm{H} 8 \mathrm{BA}$

$\mathrm{C} 4 \mathrm{~B}-\mathrm{C} 8 \mathrm{~B}-\mathrm{H} 8 \mathrm{BB}$

$\mathrm{H} 8 \mathrm{BA}-\mathrm{C} 8 \mathrm{~B}-\mathrm{H} 8 \mathrm{BB}$

$\mathrm{C} 4 \mathrm{~B}-\mathrm{C} 8 \mathrm{~B}-\mathrm{H} 8 \mathrm{BC}$

$\mathrm{H} 8 \mathrm{BA}-\mathrm{C} 8 \mathrm{~B}-\mathrm{H} 8 \mathrm{BC}$

$\mathrm{H} 8 \mathrm{BB}-\mathrm{C} 8 \mathrm{~B}-\mathrm{H} 8 \mathrm{BC}$

$\mathrm{C} 11-\mathrm{C} 5 \mathrm{~A}-\mathrm{C} 6 \mathrm{~A}-\mathrm{C} 8 \mathrm{~A}$
$\mathrm{C} 6 \mathrm{~B}-\mathrm{C} 1 \mathrm{~B}-\mathrm{C} 2 \mathrm{~B}-\mathrm{C} 3 \mathrm{~B}$
$\mathrm{C} 7 \mathrm{~B}-\mathrm{C} 1 \mathrm{~B}-\mathrm{C} 2 \mathrm{~B}-\mathrm{C} 3 \mathrm{~B}$
$\mathrm{C} 1 \mathrm{~B}-\mathrm{C} 2 \mathrm{~B}-\mathrm{C} 3 \mathrm{~B}-\mathrm{C} 4 \mathrm{~B}$
$\mathrm{C} 2 \mathrm{~B}-\mathrm{C} 3 \mathrm{~B}-\mathrm{C} 4 \mathrm{~B}-\mathrm{C} 5 \mathrm{~B}$
$\mathrm{C} 2 \mathrm{~B}-\mathrm{C} 3 \mathrm{~B}-\mathrm{C} 4 \mathrm{~B}-\mathrm{C} 8 \mathrm{~B}$
$\mathrm{C} 3 \mathrm{~B}-\mathrm{C} 4 \mathrm{~B}-\mathrm{C} 5 \mathrm{~B}-\mathrm{C} 6 \mathrm{~B}$
$\mathrm{C} 8 \mathrm{~B}-\mathrm{C} 4 \mathrm{~B}-\mathrm{C} 5 \mathrm{~B}-\mathrm{C} 6 \mathrm{~B}$
$\mathrm{C} 4 \mathrm{~B}-\mathrm{C} 5 \mathrm{~B}-\mathrm{C} 6 \mathrm{~B}-\mathrm{C} 1 \mathrm{~B}$
$\mathrm{C} 2 \mathrm{~B}-\mathrm{C} 1 \mathrm{~B}-\mathrm{C} 6 \mathrm{~B}-\mathrm{C} 5 \mathrm{~B}$
$\mathrm{C} 7 \mathrm{~B}-\mathrm{C} 1 \mathrm{~B}-\mathrm{C} 6 \mathrm{~B}-\mathrm{C} 5 \mathrm{~B}$
$\mathrm{C} 6 \mathrm{~B}-\mathrm{C} 1 \mathrm{~B}-\mathrm{C} 7 \mathrm{~B}-\mathrm{O} 2 \mathrm{~B}$
$\mathrm{C} 2 \mathrm{~B}-\mathrm{C} 1 \mathrm{~B}-\mathrm{C} 7 \mathrm{~B}-\mathrm{O} 2 \mathrm{~B}$
$\mathrm{C} 6 \mathrm{~B}-\mathrm{C} 1 \mathrm{~B}-\mathrm{C} 7 \mathrm{~B}-\mathrm{O} 1 \mathrm{~B}$
$\mathrm{C} 2 \mathrm{~B}-\mathrm{C} 1 \mathrm{~B}-\mathrm{C} 7 \mathrm{~B}-\mathrm{O} 1 \mathrm{~B}$
109.5

109.5

109.5

109.5

109.5

109.5

$-0.5(3)$

$0.5(3)$

$-179.18(19)$

$0.3(3)$

$-1.1(3)$

177.7 (2)

$1.2(3)$

$-177.6(2)$

$-0.4(3)$

$-0.4(3)$

$179.21(19)$

$179.5(2)$

$-0.8(3)$

$-0.7(3)$

178.94 (19)

Hydrogen-bond geometry $\left(A,{ }^{\circ}\right)$

\begin{tabular}{lllll}
\hline$D-\mathrm{H}^{\cdots} A$ & $D-\mathrm{H}$ & $\mathrm{H} \cdots A$ & $D \cdots A$ & $D-\mathrm{H} \cdots A$ \\
\hline $\mathrm{N} 4 A-\mathrm{H} 41 \cdots \mathrm{O} 2 B$ & $0.79(3)$ & $2.17(3)$ & $2.958(2)$ & $178(3)$ \\
$\mathrm{N} 4 A-\mathrm{H} 42 \cdots \mathrm{N} 1 A^{\mathrm{i}}$ & $0.91(3)$ & $2.18(3)$ & $3.034(2)$ & $156(2)$ \\
$\mathrm{O} 1 B-\mathrm{H} 1 B \cdots \mathrm{N} 3 A$ & $0.90(4)$ & $1.74(4)$ & $2.630(2)$ & $171(4)$ \\
\hline
\end{tabular}

Symmetry code: (i) $x,-y+3 / 2, z+1 / 2$.

4-Amino-5-chloro-2,6-dimethylpyrimidine-4-aminobenzoic acid (1/1) (COCRYSTAL-10)

Crystal data

$\mathrm{C}_{6} \mathrm{H}_{8} \mathrm{ClN}_{3} \cdot \mathrm{C}_{7} \mathrm{H}_{7} \mathrm{NO}_{2}$

$M_{r}=294.74$

Monoclinic, $P 2_{1} / c$

$a=7.8899(2) \AA$

$b=13.2361(3) \AA$

$c=13.3212(3) \AA$

$\beta=101.254(2)^{\circ}$

$V=1364.40(6) \AA^{3}$

$Z=4$

\section{Data collection}

Agilent SuperNova Dual Source diffractometer with an Atlas detector

Radiation source: sealed X-ray tube
$F(000)=616$

$D_{\mathrm{x}}=1.435 \mathrm{Mg} \mathrm{m}^{-3}$

$\mathrm{Cu} K \alpha$ radiation, $\lambda=1.54184 \AA$

Cell parameters from 4256 reflections

$\theta=3.3-76.1^{\circ}$

$\mu=2.56 \mathrm{~mm}^{-1}$

$T=120 \mathrm{~K}$

Chunk, colorless

$0.41 \times 0.35 \times 0.24 \mathrm{~mm}$

Detector resolution: 10.6501 pixels $\mathrm{mm}^{-1}$ $\omega$ scans 
Absorption correction: multi-scan

(CrysAlis PRO; Agilent, 2014)

$T_{\text {min }}=0.828, T_{\text {max }}=1.000$

6214 measured reflections

2823 independent reflections

2749 reflections with $I>2 \sigma(I)$

Refinement

Refinement on $F^{2}$

Least-squares matrix: full

$R\left[F^{2}>2 \sigma\left(F^{2}\right)\right]=0.036$

$w R\left(F^{2}\right)=0.097$

$S=1.04$

2823 reflections

203 parameters

0 restraints

$$
\begin{aligned}
& R_{\text {int }}=0.017 \\
& \theta_{\max }=76.7^{\circ}, \theta_{\min }=4.8^{\circ} \\
& h=-9 \rightarrow 9 \\
& k=-16 \rightarrow 15 \\
& l=-14 \rightarrow 16
\end{aligned}
$$

Hydrogen site location: mixed

$\mathrm{H}$ atoms treated by a mixture of independent and constrained refinement

$w=1 /\left[\sigma^{2}\left(F_{\mathrm{o}}^{2}\right)+(0.0596 P)^{2}+0.5634 P\right]$ where $P=\left(F_{\mathrm{o}}^{2}+2 F_{\mathrm{c}}{ }^{2}\right) / 3$

$(\Delta / \sigma)_{\max }=0.002$

$\Delta \rho_{\max }=0.27 \mathrm{e} \AA^{-3}$

$\Delta \rho_{\min }=-0.38$ e $\AA^{-3}$

Special details

Geometry. All esds (except the esd in the dihedral angle between two 1.s. planes) are estimated using the full covariance matrix. The cell esds are taken into account individually in the estimation of esds in distances, angles and torsion angles; correlations between esds in cell parameters are only used when they are defined by crystal symmetry. An approximate (isotropic) treatment of cell esds is used for estimating esds involving l.s. planes.

Fractional atomic coordinates and isotropic or equivalent isotropic displacement parameters $\left(\AA^{2}\right)$

\begin{tabular}{lllll}
\hline & $x$ & $y$ & $z$ & $U_{\text {iso }} / U_{\text {eq }}$ \\
\hline C11 & $1.05069(4)$ & $0.86469(2)$ & $0.60646(2)$ & $0.02693(12)$ \\
N1A & $0.93400(14)$ & $0.71297(8)$ & $0.34395(8)$ & $0.0197(2)$ \\
C2A & $0.86103(17)$ & $0.63206(10)$ & $0.37710(10)$ & $0.0193(3)$ \\
N3A & $0.84090(14)$ & $0.61587(8)$ & $0.47345(8)$ & $0.0185(2)$ \\
N4A & $0.86802(16)$ & $0.67391(9)$ & $0.63872(9)$ & $0.0230(3)$ \\
H4A1 & $0.817(2)$ & $0.6176(15)$ & $0.6497(14)$ & $0.028(4)^{*}$ \\
H4A2 & $0.897(3)$ & $0.7162(16)$ & $0.6858(16)$ & $0.037(5)^{*}$ \\
C4A & $0.89549(16)$ & $0.68774(10)$ & $0.54425(10)$ & $0.0184(3)$ \\
C5A & $0.97806(16)$ & $0.77421(9)$ & $0.51419(10)$ & $0.0187(3)$ \\
C6A & $0.99586(16)$ & $0.78486(10)$ & $0.41444(10)$ & $0.0184(3)$ \\
C7A & $0.7941(2)$ & $0.55104(11)$ & $0.30121(11)$ & $0.0265(3)$ \\
H7AA & 0.8015 & 0.5740 & 0.2322 & $0.040^{*}$ \\
H7AB & 0.6734 & 0.5364 & 0.3040 & $0.040^{*}$ \\
H7AC & 0.8637 & 0.4898 & 0.3177 & $0.040^{*}$ \\
C8A & $1.07803(18)$ & $0.87473(10)$ & $0.37573(11)$ & $0.0226(3)$ \\
H8AA & 1.1481 & 0.8527 & 0.3266 & $0.034^{*}$ \\
H8AB & 1.1519 & 0.9092 & 0.4332 & $0.034^{*}$ \\
H8AC & 0.9880 & 0.9212 & 0.3420 & $0.034^{*}$ \\
O1B & $0.70377(14)$ & $0.43906(8)$ & $0.50497(8)$ & $0.0281(2)$ \\
H1B & $0.748(3)$ & $0.501(2)$ & $0.5011(19)$ & $0.052(6)^{*}$ \\
O2B & $0.7164(14)$ & $0.47720(7)$ & $0.66954(8)$ & $0.0260(2)$ \\
N1B & $0.37076(18)$ & $0.03549(10)$ & $0.62891(11)$ & $0.0289(3)$ \\
H1B1 & $0.352(3)$ & $0.0138(16)$ & $0.6885(17)$ & $0.035(5)^{*}$ \\
H1B2 & $0.329(3)$ & $0.0044(17)$ & $0.5724(18)$ & $0.041(5)^{*}$ \\
& & & &
\end{tabular}




$\begin{array}{lllll}\text { C1B } & 0.59620(17) & 0.31826(10) & 0.60438(10) & 0.0201(3) \\ \text { C2B } & 0.57168(17) & 0.28210(10) & 0.69932(10) & 0.0222(3) \\ \text { H2BA } & 0.6064 & 0.3224 & 0.7588 & 0.027^{*} \\ \text { C3B } & 0.49760(18) & 0.18860(11) & 0.70760(10) & 0.0232(3) \\ \text { H3BA } & 0.4834 & 0.1649 & 0.7728 & 0.028^{*} \\ \text { C4B } & 0.44307(17) & 0.12835(10) & 0.62039(11) & 0.0208(3) \\ \text { C5B } & 0.46641(18) & 0.16502(10) & 0.52511(10) & 0.0224(3) \\ \text { H5BA } & 0.4293 & 0.1257 & 0.4652 & 0.027^{*} \\ \text { C6B } & 0.54303(17) & 0.25799(11) & 0.51799(10) & 0.0225(3) \\ \text { H6BA } & 0.5598 & 0.2813 & 0.4532 & 0.027^{*} \\ \text { C7B } & 0.67560(17) & 0.41846(10) & 0.59684(10) & 0.0211(3)\end{array}$

Atomic displacement parameters $\left(\AA^{2}\right)$

\begin{tabular}{lllllll}
\hline & $U^{11}$ & $U^{22}$ & $U^{33}$ & $U^{12}$ & $U^{13}$ & $U^{23}$ \\
\hline C11 & $0.0366(2)$ & $0.02105(19)$ & $0.02460(19)$ & $-0.01009(12)$ & $0.00947(14)$ & $-0.00687(11)$ \\
N1A & $0.0223(5)$ & $0.0179(5)$ & $0.0195(5)$ & $-0.0010(4)$ & $0.0059(4)$ & $-0.0004(4)$ \\
C2A & $0.0207(6)$ & $0.0180(6)$ & $0.0198(6)$ & $0.0001(5)$ & $0.0055(5)$ & $-0.0016(5)$ \\
N3A & $0.0217(5)$ & $0.0154(5)$ & $0.0191(5)$ & $-0.0017(4)$ & $0.0055(4)$ & $-0.0010(4)$ \\
N4A & $0.0335(6)$ & $0.0181(6)$ & $0.0181(6)$ & $-0.0068(5)$ & $0.0070(5)$ & $-0.0015(4)$ \\
C4A & $0.0194(6)$ & $0.0160(6)$ & $0.0197(6)$ & $0.0016(4)$ & $0.0037(5)$ & $-0.0001(5)$ \\
C5A & $0.0201(6)$ & $0.0154(6)$ & $0.0207(6)$ & $-0.0007(5)$ & $0.0046(5)$ & $-0.0015(5)$ \\
C6A & $0.0165(5)$ & $0.0163(6)$ & $0.0225(6)$ & $0.0015(4)$ & $0.0042(5)$ & $0.0010(5)$ \\
C7A & $0.0356(7)$ & $0.0226(7)$ & $0.0228(7)$ & $-0.0065(6)$ & $0.0095(5)$ & $-0.0056(5)$ \\
C8A & $0.0256(6)$ & $0.0189(6)$ & $0.0243(7)$ & $-0.0025(5)$ & $0.0074(5)$ & $0.0023(5)$ \\
O1B & $0.0412(6)$ & $0.0209(5)$ & $0.0248(5)$ & $-0.0089(4)$ & $0.0130(4)$ & $-0.0005(4)$ \\
O2B & $0.0358(5)$ & $0.0186(5)$ & $0.0255(5)$ & $-0.0047(4)$ & $0.0104(4)$ & $-0.0020(4)$ \\
N1B & $0.0371(7)$ & $0.0233(6)$ & $0.0274(7)$ & $-0.0114(5)$ & $0.0093(5)$ & $-0.0017(5)$ \\
C1B & $0.0200(6)$ & $0.0171(6)$ & $0.0241(6)$ & $-0.0002(5)$ & $0.0064(5)$ & $0.0006(5)$ \\
C2B & $0.0248(6)$ & $0.0205(6)$ & $0.0221(6)$ & $-0.0012(5)$ & $0.0064(5)$ & $-0.0015(5)$ \\
C3B & $0.0265(7)$ & $0.0225(6)$ & $0.0221(6)$ & $-0.0024(5)$ & $0.0088(5)$ & $0.0006(5)$ \\
C4B & $0.0177(6)$ & $0.0191(6)$ & $0.0263(7)$ & $-0.0008(5)$ & $0.0062(5)$ & $0.0006(5)$ \\
C5B & $0.0242(6)$ & $0.0211(6)$ & $0.0215(6)$ & $-0.0023(5)$ & $0.0034(5)$ & $-0.0028(5)$ \\
C6B & $0.0250(6)$ & $0.0222(6)$ & $0.0210(6)$ & $-0.0002(5)$ & $0.0062(5)$ & $0.0020(5)$ \\
C7B & $0.0225(6)$ & $0.0185(6)$ & $0.0234(6)$ & $0.0012(5)$ & $0.0069(5)$ & $0.0014(5)$ \\
& & & & & & \\
\hline
\end{tabular}

Geometric parameters $\left(\stackrel{A}{\circ}{ }^{\circ}\right)$

\begin{tabular}{llll}
\hline $\mathrm{C} 11-\mathrm{C} 5 \mathrm{~A}$ & $1.7316(13)$ & $\mathrm{O} 1 \mathrm{~B}-\mathrm{C} 7 \mathrm{~B}$ & $1.3145(17)$ \\
$\mathrm{N} 1 \mathrm{~A}-\mathrm{C} 2 \mathrm{~A}$ & $1.3314(17)$ & $\mathrm{O} 1 \mathrm{~B}-\mathrm{H} 1 \mathrm{~B}$ & $0.90(3)$ \\
$\mathrm{N} 1 \mathrm{~A}-\mathrm{C} 6 \mathrm{~A}$ & $1.3589(17)$ & $\mathrm{O} 2 \mathrm{~B}-\mathrm{C} 7 \mathrm{~B}$ & $1.2311(17)$ \\
$\mathrm{C} 2 \mathrm{~A}-\mathrm{N} 3 \mathrm{~A}$ & $1.3410(17)$ & $\mathrm{N} 1 \mathrm{~B}-\mathrm{C} 4 \mathrm{~B}$ & $1.3691(17)$ \\
$\mathrm{C} 2 \mathrm{~A}-\mathrm{C} 7 \mathrm{~A}$ & $1.4975(18)$ & $\mathrm{N} 1 \mathrm{~B}-\mathrm{H} 1 \mathrm{~B} 1$ & $0.89(2)$ \\
$\mathrm{N} 3 \mathrm{~A}-\mathrm{C} 4 \mathrm{~A}$ & $1.3497(17)$ & $\mathrm{N} 1 \mathrm{~B}-\mathrm{H} 1 \mathrm{~B} 2$ & $0.86(2)$ \\
$\mathrm{N} 4 \mathrm{~A}-\mathrm{C} 4 \mathrm{~A}$ & $1.3307(17)$ & $\mathrm{C} 1 \mathrm{~B}-\mathrm{C} 6 \mathrm{~B}$ & $1.3958(19)$ \\
$\mathrm{N} 4 \mathrm{~A}-\mathrm{H} 4 \mathrm{~A} 1$ & $0.87(2)$ & $\mathrm{C} 1 \mathrm{~B}-\mathrm{C} 2 \mathrm{~B}$ & $1.4005(19)$ \\
$\mathrm{N} 4 \mathrm{~A}-\mathrm{H} 4 \mathrm{~A} 2$ & $0.84(2)$ & $\mathrm{C} 1 \mathrm{~B}-\mathrm{C} 7 \mathrm{~B}$ & $1.4785(18)$ \\
$\mathrm{C} 4 \mathrm{~A}-\mathrm{C} 5 \mathrm{~A}$ & $1.4129(18)$ & $\mathrm{C} 2 \mathrm{~B}-\mathrm{C} 3 \mathrm{~B}$ & $1.3823(19)$
\end{tabular}




$$
\begin{aligned}
& \mathrm{C} 5 \mathrm{~A}-\mathrm{C} 6 \mathrm{~A} \\
& \mathrm{C} 6 \mathrm{~A}-\mathrm{C} 8 \mathrm{~A} \\
& \mathrm{C} 7 \mathrm{~A}-\mathrm{H} 7 \mathrm{AA} \\
& \mathrm{C} 7 \mathrm{~A}-\mathrm{H} 7 \mathrm{AB} \\
& \mathrm{C} 7 \mathrm{~A}-\mathrm{H} 7 \mathrm{AC} \\
& \mathrm{C} 8 \mathrm{~A}-\mathrm{H} 8 \mathrm{AA} \\
& \mathrm{C} 8 \mathrm{~A}-\mathrm{H} 8 \mathrm{AB} \\
& \text { C8A-H8AC }
\end{aligned}
$$$$
\mathrm{C} 2 \mathrm{~A}-\mathrm{N} 1 \mathrm{~A}-\mathrm{C} 6 \mathrm{~A}
$$$$
\mathrm{N} 1 \mathrm{~A}-\mathrm{C} 2 \mathrm{~A}-\mathrm{N} 3 \mathrm{~A}
$$$$
\mathrm{N} 1 \mathrm{~A}-\mathrm{C} 2 \mathrm{~A}-\mathrm{C} 7 \mathrm{~A}
$$$$
\mathrm{N} 3 \mathrm{~A}-\mathrm{C} 2 \mathrm{~A}-\mathrm{C} 7 \mathrm{~A}
$$$$
\mathrm{C} 2 \mathrm{~A}-\mathrm{N} 3 \mathrm{~A}-\mathrm{C} 4 \mathrm{~A}
$$$$
\mathrm{C} 4 \mathrm{~A}-\mathrm{N} 4 \mathrm{~A}-\mathrm{H} 4 \mathrm{~A} 1
$$$$
\mathrm{C} 4 \mathrm{~A}-\mathrm{N} 4 \mathrm{~A}-\mathrm{H} 4 \mathrm{~A} 2
$$$$
\mathrm{H} 4 \mathrm{~A} 1-\mathrm{N} 4 \mathrm{~A}-\mathrm{H} 4 \mathrm{~A} 2
$$$$
\mathrm{N} 4 \mathrm{~A}-\mathrm{C} 4 \mathrm{~A}-\mathrm{N} 3 \mathrm{~A}
$$$$
\mathrm{N} 4 \mathrm{~A}-\mathrm{C} 4 \mathrm{~A}-\mathrm{C} 5 \mathrm{~A}
$$$$
\mathrm{N} 3 \mathrm{~A}-\mathrm{C} 4 \mathrm{~A}-\mathrm{C} 5 \mathrm{~A}
$$$$
\mathrm{C} 6 \mathrm{~A}-\mathrm{C} 5 \mathrm{~A}-\mathrm{C} 4 \mathrm{~A}
$$$$
\mathrm{C} 6 \mathrm{~A}-\mathrm{C} 5 \mathrm{~A}-\mathrm{Cl1}
$$$$
\mathrm{C} 4 \mathrm{~A}-\mathrm{C} 5 \mathrm{~A}-\mathrm{C} 11
$$$$
\mathrm{N} 1 \mathrm{~A}-\mathrm{C} 6 \mathrm{~A}-\mathrm{C} 5 \mathrm{~A}
$$$$
\mathrm{N} 1 \mathrm{~A}-\mathrm{C} 6 \mathrm{~A}-\mathrm{C} 8 \mathrm{~A}
$$$$
\mathrm{C} 5 \mathrm{~A}-\mathrm{C} 6 \mathrm{~A}-\mathrm{C} 8 \mathrm{~A}
$$$$
\mathrm{C} 2 \mathrm{~A}-\mathrm{C} 7 \mathrm{~A}-\mathrm{H} 7 \mathrm{AA}
$$$$
\mathrm{C} 2 \mathrm{~A}-\mathrm{C} 7 \mathrm{~A}-\mathrm{H} 7 \mathrm{AB}
$$$$
\mathrm{H} 7 \mathrm{AA}-\mathrm{C} 7 \mathrm{~A}-\mathrm{H} 7 \mathrm{AB}
$$$$
\mathrm{C} 2 \mathrm{~A}-\mathrm{C} 7 \mathrm{~A}-\mathrm{H} 7 \mathrm{AC}
$$$$
\text { H7AA-C7A-H7AC }
$$$$
\mathrm{H} 7 \mathrm{AB}-\mathrm{C} 7 \mathrm{~A}-\mathrm{H} 7 \mathrm{AC}
$$$$
\text { C6A-C8A-H8AA }
$$$$
\mathrm{C} 6 \mathrm{~A}-\mathrm{C} 8 \mathrm{~A}-\mathrm{H} 8 \mathrm{AB}
$$$$
\text { H8AA- } \mathrm{C} 8 \mathrm{~A}-\mathrm{H} 8 \mathrm{AB}
$$$$
\mathrm{C} 6 \mathrm{~A}-\mathrm{C} 8 \mathrm{~A}-\mathrm{H} 8 \mathrm{AC}
$$$$
\mathrm{C} 6 \mathrm{~A}-\mathrm{N} 1 \mathrm{~A}-\mathrm{C} 2 \mathrm{~A}-\mathrm{N} 3 \mathrm{~A}
$$$$
\mathrm{C} 6 \mathrm{~A}-\mathrm{N} 1 \mathrm{~A}-\mathrm{C} 2 \mathrm{~A}-\mathrm{C} 7 \mathrm{~A}
$$$$
\mathrm{N} 1 \mathrm{~A}-\mathrm{C} 2 \mathrm{~A}-\mathrm{N} 3 \mathrm{~A}-\mathrm{C} 4 \mathrm{~A}
$$$$
\mathrm{C} 7 \mathrm{~A}-\mathrm{C} 2 \mathrm{~A}-\mathrm{N} 3 \mathrm{~A}-\mathrm{C} 4 \mathrm{~A}
$$$$
\mathrm{C} 2 \mathrm{~A}-\mathrm{N} 3 \mathrm{~A}-\mathrm{C} 4 \mathrm{~A}-\mathrm{N} 4 \mathrm{~A}
$$$$
\mathrm{C} 2 \mathrm{~A}-\mathrm{N} 3 \mathrm{~A}-\mathrm{C} 4 \mathrm{~A}-\mathrm{C} 5 \mathrm{~A}
$$$$
\mathrm{N} 4 \mathrm{~A}-\mathrm{C} 4 \mathrm{~A}-\mathrm{C} 5 \mathrm{~A}-\mathrm{C} 6 \mathrm{~A}
$$$$
\mathrm{N} 3 \mathrm{~A}-\mathrm{C} 4 \mathrm{~A}-\mathrm{C} 5 \mathrm{~A}-\mathrm{C} 6 \mathrm{~A}
$$$$
\mathrm{N} 4 \mathrm{~A}-\mathrm{C} 4 \mathrm{~A}-\mathrm{C} 5 \mathrm{~A}-\mathrm{C} 11
$$$$
\mathrm{N} 3 \mathrm{~A}-\mathrm{C} 4 \mathrm{~A}-\mathrm{C} 5 \mathrm{~A}-\mathrm{C} 11
$$$$
\mathrm{C} 2 \mathrm{~A}-\mathrm{N} 1 \mathrm{~A}-\mathrm{C} 6 \mathrm{~A}-\mathrm{C} 5 \mathrm{~A}
$$

$1.3704(18)$

1.4939 (17)

0.9800

0.9800

0.9800

0.9800

0.9800

0.9800

$117.02(11)$

$125.85(12)$

$118.06(12)$

$116.08(11)$

118.35 (11)

$116.2(13)$

$123.1(14)$

120.7 (19)

$118.37(12)$

$123.28(12)$

$118.35(11)$

$119.89(12)$

$122.33(10)$

$117.77(10)$

$120.47(12)$

$116.14(11)$

$123.37(12)$

109.5

109.5

109.5

109.5

109.5

109.5

109.5

109.5

109.5

109.5

$1.0(2)$

$-179.14(12)$

1.3 (2)

$-178.49(12)$

$176.81(12)$

$-2.83(18)$

$-177.52(12)$

$2.10(19)$

$1.49(18)$

$-178.90(9)$

$-1.78(18)$

$$
\begin{aligned}
& \mathrm{C} 2 \mathrm{~B}-\mathrm{H} 2 \mathrm{BA} \\
& \mathrm{C} 3 \mathrm{~B}-\mathrm{C} 4 \mathrm{~B} \\
& \mathrm{C} 3 \mathrm{~B}-\mathrm{H} 3 \mathrm{BA} \\
& \mathrm{C} 4 \mathrm{~B}-\mathrm{C} 5 \mathrm{~B} \\
& \mathrm{C} 5 \mathrm{~B}-\mathrm{C} 6 \mathrm{~B} \\
& \mathrm{C} 5 \mathrm{~B}-\mathrm{H} 5 \mathrm{BA} \\
& \mathrm{C} 6 \mathrm{~B}-\mathrm{H} 6 \mathrm{BA}
\end{aligned}
$$

H8AA- $88 \mathrm{~A}-\mathrm{H} 8 \mathrm{AC}$

$\mathrm{H} 8 \mathrm{AB}-\mathrm{C} 8 \mathrm{~A}-\mathrm{H} 8 \mathrm{AC}$

$\mathrm{C} 7 \mathrm{~B}-\mathrm{O} 1 \mathrm{~B}-\mathrm{H} 1 \mathrm{~B}$

$\mathrm{C} 4 \mathrm{~B}-\mathrm{N} 1 \mathrm{~B}-\mathrm{H} 1 \mathrm{~B} 1$

$\mathrm{C} 4 \mathrm{~B}-\mathrm{N} 1 \mathrm{~B}-\mathrm{H} 1 \mathrm{~B} 2$

H1B1-N1B-H1B2

$\mathrm{C} 6 \mathrm{~B}-\mathrm{C} 1 \mathrm{~B}-\mathrm{C} 2 \mathrm{~B}$

$\mathrm{C} 6 \mathrm{~B}-\mathrm{C} 1 \mathrm{~B}-\mathrm{C} 7 \mathrm{~B}$

$\mathrm{C} 2 \mathrm{~B}-\mathrm{C} 1 \mathrm{~B}-\mathrm{C} 7 \mathrm{~B}$

$\mathrm{C} 3 \mathrm{~B}-\mathrm{C} 2 \mathrm{~B}-\mathrm{C} 1 \mathrm{~B}$

$\mathrm{C} 3 \mathrm{~B}-\mathrm{C} 2 \mathrm{~B}-\mathrm{H} 2 \mathrm{BA}$

$\mathrm{C} 1 \mathrm{~B}-\mathrm{C} 2 \mathrm{~B}-\mathrm{H} 2 \mathrm{BA}$

$\mathrm{C} 2 \mathrm{~B}-\mathrm{C} 3 \mathrm{~B}-\mathrm{C} 4 \mathrm{~B}$

$\mathrm{C} 2 \mathrm{~B}-\mathrm{C} 3 \mathrm{~B}-\mathrm{H} 3 \mathrm{BA}$

$\mathrm{C} 4 \mathrm{~B}-\mathrm{C} 3 \mathrm{~B}-\mathrm{H} 3 \mathrm{BA}$

$\mathrm{N} 1 \mathrm{~B}-\mathrm{C} 4 \mathrm{~B}-\mathrm{C} 5 \mathrm{~B}$

$\mathrm{N} 1 \mathrm{~B}-\mathrm{C} 4 \mathrm{~B}-\mathrm{C} 3 \mathrm{~B}$

$\mathrm{C} 5 \mathrm{~B}-\mathrm{C} 4 \mathrm{~B}-\mathrm{C} 3 \mathrm{~B}$

$\mathrm{C} 6 \mathrm{~B}-\mathrm{C} 5 \mathrm{~B}-\mathrm{C} 4 \mathrm{~B}$

$\mathrm{C} 6 \mathrm{~B}-\mathrm{C} 5 \mathrm{~B}-\mathrm{H} 5 \mathrm{BA}$

$\mathrm{C} 4 \mathrm{~B}-\mathrm{C} 5 \mathrm{~B}-\mathrm{H} 5 \mathrm{BA}$

$\mathrm{C} 5 \mathrm{~B}-\mathrm{C} 6 \mathrm{~B}-\mathrm{C} 1 \mathrm{~B}$

$\mathrm{C} 5 \mathrm{~B}-\mathrm{C} 6 \mathrm{~B}-\mathrm{H} 6 \mathrm{BA}$

$\mathrm{C} 1 \mathrm{~B}-\mathrm{C} 6 \mathrm{~B}-\mathrm{H} 6 \mathrm{BA}$

$\mathrm{O} 2 \mathrm{~B}-\mathrm{C} 7 \mathrm{~B}-\mathrm{O} 1 \mathrm{~B}$

$\mathrm{O} 2 \mathrm{~B}-\mathrm{C} 7 \mathrm{~B}-\mathrm{C} 1 \mathrm{~B}$

$\mathrm{O} 1 \mathrm{~B}-\mathrm{C} 7 \mathrm{~B}-\mathrm{C} 1 \mathrm{~B}$

$\mathrm{C} 11-\mathrm{C} 5 \mathrm{~A}-\mathrm{C} 6 \mathrm{~A}-\mathrm{C} 8 \mathrm{~A}$

$\mathrm{C} 6 \mathrm{~B}-\mathrm{C} 1 \mathrm{~B}-\mathrm{C} 2 \mathrm{~B}-\mathrm{C} 3 \mathrm{~B}$

$\mathrm{C} 7 \mathrm{~B}-\mathrm{C} 1 \mathrm{~B}-\mathrm{C} 2 \mathrm{~B}-\mathrm{C} 3 \mathrm{~B}$

$\mathrm{C} 1 \mathrm{~B}-\mathrm{C} 2 \mathrm{~B}-\mathrm{C} 3 \mathrm{~B}-\mathrm{C} 4 \mathrm{~B}$

$\mathrm{C} 2 \mathrm{~B}-\mathrm{C} 3 \mathrm{~B}-\mathrm{C} 4 \mathrm{~B}-\mathrm{N} 1 \mathrm{~B}$

$\mathrm{C} 2 \mathrm{~B}-\mathrm{C} 3 \mathrm{~B}-\mathrm{C} 4 \mathrm{~B}-\mathrm{C} 5 \mathrm{~B}$

$\mathrm{N} 1 \mathrm{~B}-\mathrm{C} 4 \mathrm{~B}-\mathrm{C} 5 \mathrm{~B}-\mathrm{C} 6 \mathrm{~B}$

$\mathrm{C} 3 \mathrm{~B}-\mathrm{C} 4 \mathrm{~B}-\mathrm{C} 5 \mathrm{~B}-\mathrm{C} 6 \mathrm{~B}$

$\mathrm{C} 4 \mathrm{~B}-\mathrm{C} 5 \mathrm{~B}-\mathrm{C} 6 \mathrm{~B}-\mathrm{C} 1 \mathrm{~B}$

$\mathrm{C} 2 \mathrm{~B}-\mathrm{C} 1 \mathrm{~B}-\mathrm{C} 6 \mathrm{~B}-\mathrm{C} 5 \mathrm{~B}$

$\mathrm{C} 7 \mathrm{~B}-\mathrm{C} 1 \mathrm{~B}-\mathrm{C} 6 \mathrm{~B}-\mathrm{C} 5 \mathrm{~B}$
0.9500

$1.4056(19)$

0.9500

$1.4041(19)$

$1.3824(19)$

0.9500

0.9500

109.5

109.5

$112.3(16)$

$120.9(13)$

116.6 (14)

$122(2)$

$118.49(12)$

$121.32(12)$

$120.19(12)$

$120.87(13)$

119.6

119.6

120.53 (13)

119.7

119.7

121.03 (13)

$120.40(13)$

$118.56(12)$

$120.40(12)$

119.8

119.8

121.15 (12)

119.4

119.4

$123.12(12)$

$123.29(12)$

$113.59(12)$

$-0.28(18)$

0.4 (2)

$179.99(12)$

-0.9 (2)

$179.58(13)$

0.3 (2)

-178.59 (13)

0.6 (2)

$-1.1(2)$

$0.6(2)$

$-178.99(12)$ 


$\begin{array}{llll}\mathrm{C} 2 \mathrm{~A}-\mathrm{N} 1 \mathrm{~A}-\mathrm{C} 6 \mathrm{~A}-\mathrm{C} 8 \mathrm{~A} & 179.69(11) & \mathrm{C} 6 \mathrm{~B}-\mathrm{C} 1 \mathrm{~B}-\mathrm{C} 7 \mathrm{~B}-\mathrm{O} 2 \mathrm{~B} & 174.06(13) \\ \mathrm{C} 4 \mathrm{~A}-\mathrm{C} 5 \mathrm{~A}-\mathrm{C} 6 \mathrm{~A}-\mathrm{N} 1 \mathrm{~A} & 0.26(19) & \mathrm{C} 2 \mathrm{~B}-\mathrm{C} 1 \mathrm{~B}-\mathrm{C} 7 \mathrm{~B}-\mathrm{O} 2 \mathrm{~B} & -5.5(2) \\ \mathrm{C} 11-\mathrm{C} 5 \mathrm{~A}-\mathrm{C} 6 \mathrm{~A}-\mathrm{N} 1 \mathrm{~A} & -178.69(9) & \mathrm{C} 6 \mathrm{~B}-\mathrm{C} 1 \mathrm{~B}-\mathrm{C} 7 \mathrm{~B}-\mathrm{O} 1 \mathrm{~B} & -5.78(19) \\ \mathrm{C} 4 \mathrm{~A}-\mathrm{C} 5 \mathrm{~A}-\mathrm{C} 6 \mathrm{~A}-\mathrm{C} 8 \mathrm{~A} & 178.68(12) & \mathrm{C} 2 \mathrm{~B}-\mathrm{C} 1 \mathrm{~B}-\mathrm{C} 7 \mathrm{~B}-\mathrm{O} 1 \mathrm{~B} & 174.67(12)\end{array}$

Hydrogen-bond geometry $\left(A,{ }^{\circ}\right)$

\begin{tabular}{lllll}
\hline$D-\mathrm{H} \cdots A$ & $D-\mathrm{H}$ & $\mathrm{H} \cdots A$ & $D \cdots A$ & $D-\mathrm{H} \cdots A$ \\
\hline $\mathrm{N} 4 A-\mathrm{H} 4 A 1 \cdots \mathrm{O} 2 B$ & $0.87(2)$ & $2.07(2)$ & $2.9441(15)$ & $174.7(18)$ \\
$\mathrm{N} 4 A-\mathrm{H} 4 A 2 \cdots \mathrm{N} 1 A^{\mathrm{i}}$ & $0.84(2)$ & $2.27(2)$ & $3.0710(16)$ & $159.5(19)$ \\
$\mathrm{C} 7 A-\mathrm{H} 7 A A \cdots \mathrm{C} 11^{\mathrm{ii}}$ & 0.98 & 2.94 & $3.7580(15)$ & 142 \\
$\mathrm{C} 8 A-\mathrm{H} 8 A C \cdots \mathrm{C} 11^{\mathrm{iii}}$ & 0.98 & 2.95 & $3.6163(14)$ & 127 \\
$\mathrm{O} 1 B-\mathrm{H} 1 B \cdots \mathrm{N} 3 A$ & $0.90(3)$ & $1.76(3)$ & $2.6459(15)$ & $170(2)$ \\
$\mathrm{N} 1 B-\mathrm{H} 1 B 1 \cdots \mathrm{O} 2 B^{\mathrm{iv}}$ & $0.89(2)$ & $2.10(2)$ & $2.9847(17)$ & $173.4(19)$ \\
\hline
\end{tabular}

Symmetry codes: (i) $x,-y+3 / 2, z+1 / 2$; (ii) $x,-y+3 / 2, z-1 / 2$; (iii) $-x+2,-y+2,-z+1$; (iv) $-x+1, y-1 / 2,-z+3 / 2$. 